\title{
Hyperpolarized Gas Diffusion MRI using Steady State Free Precession Pulse Sequences
}

\author{
Karen Emily Mooney \\ Hamilton, NJ
}

B.A., Drew University, 2004

\begin{abstract}
A Dissertation presented to the Graduate Faculty of the University of Virginia in Candidacy for the Degree of Doctor of Philosophy
\end{abstract}

Department of Physics

University of Virginia

May, 2012

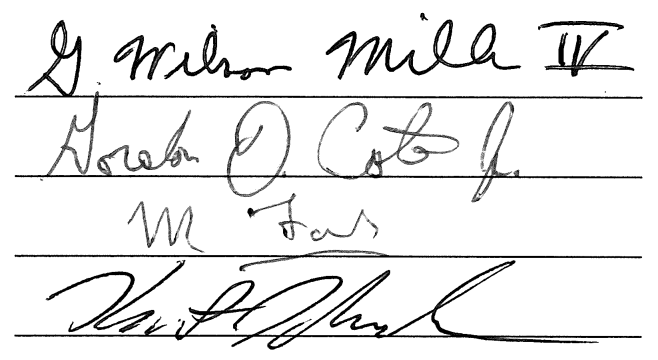




\section{Abstract}

Hyperpolarized noble gas magnetic resonance imaging (MRI) provides a unique view of the airspaces in human lungs. However, images created with this technique have a fundamental resolution limit due in part to the gas diffusion within the air spaces during the image acquisition. The process of diffusion can be used to provide a method for extracting structural information below the resolution limit, via short-time diffusion MR. In free space, the area a gas particle explores in a given amount of time (the diffusion coefficient) is a constant that does not depend on the duration over which the measurement is made. In a highly restrictive area like the lung airspaces, the diffusion coefficient varies greatly with the duration of the measurement.

For very short times measurement times, the diffusion coefficient approaches the free space value, while at longer measurement times the surrounding walls prevent the particle from traveling. This time dependence is related to the surface to volume ratio of the confining space.

The goal of this work was to develop a method of making diffusion-weighted measurements at diffusion times less than $\sim 1 \mathrm{~ms}$ to detect this time dependence in restrictive environments such as the human lung. In order to make measurements at these short times, we turned to an MRI technique known as Steady State Free Precession (SSFP). SSFP pulse sequences are coherent, which means the transverse magnetization is not zero at the application of the next RF pulse. An advantage of 
using an SSFP pulse sequence is that it produces a very high signal level on which to measure the small diffusion attenuation imparted by short-time measurements.

We developed several modifications to an SSFP pulse sequence which include diffusion sensitization, and investigated the behavior of each of these methods through the use of a magnetization simulation. We made global apparent diffusion coefficient (ADC) measurements, as well as created images with the resulting pulse sequences. In the global version, we were able to make ADC measurements over a range of diffusion times from $300-800 \mu \mathrm{s}$ in glass-bead phantoms and fit the time-dependent ADC to extract the packing volume fraction $\phi$ for each of the phantoms. Multiple diffusion-time global ADC measurements made in human subjects highlighted the differences between healthy and emphysmatic lungs. In the imaging experiments, we generated ADC maps at a diffusion time of $500 \mu \mathrm{s}$ in several human subjects. 


\section{Acknowledgements}

I would like to express my sincerest gratitude to the following people who helped me achieve this goal...

Advisors: Gordon Cates and Wilson Miller. You supported, inspired, and challenged me well longer than we all planned.

Cates-labmates: Michael Carl, Peter Dolph, Vladimir Nelyubin, Scott Rohrbaugh, Jaideep Singh, and Al Tobias. You served as teachers and friends, as well as proof it could be done. In alphabetical order, so that there are no fights.

The Radiology Research Hyperpolarized Gas Group, in particular Jaime Mata and John Mugler for their guidance and help.

The Physics Department Staff: Tammie, Dawn, Pam, Beth, Helen, and formerly Suzie. You guys make the UVa Physics Department a wonderful place to spend a graduate career. We are a truly lucky group to have you.

My family, who never stopped letting me know how proud they are of me.

And Archer, who found the right mix of supporting, distracting, and nagging me to make this result possible. 


\section{Contents}

$\begin{array}{ll}\text { Abstract } & \text { ii }\end{array}$

Acknowledgements $\quad$ iv

Table of Contents $\quad$ v

1 Introduction 1

2 Magnetic Resonance Imaging 9

2.1 Spins in a Magnetic Field . . . . . . . . . . . . . . . . 10

2.2 Nuclear Magnetic Resonance . . . . . . . . . . . . . . . . . 14

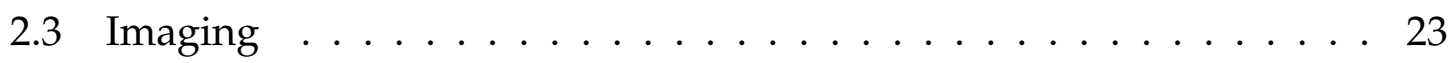

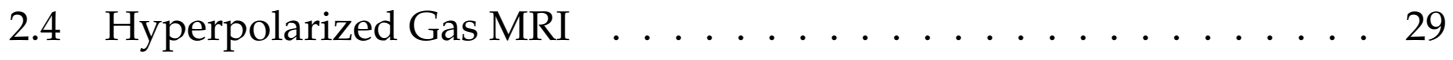

2.5 Diffusion-weighted Imaging $\ldots \ldots \ldots \ldots \ldots \ldots$

3 Hybrid Rubidium/Potassium Helium Polarizer for Medical Imaging 43

3.1 Spin-Exchange Optical Pumping . . . . . . . . . . . . . . 44

3.2 Hybrid Polarizer Design . . . . . . . . . . . . . . . . . . . 49

3.3 Lasers and Optics . . . . . . . . . . . . . . . . . . . 53

3.4 Hybrid SEOP $\ldots \ldots \ldots \ldots \ldots \ldots \ldots \ldots$

3.5 Measurement and Calibration . . . . . . . . . . . . . . 58

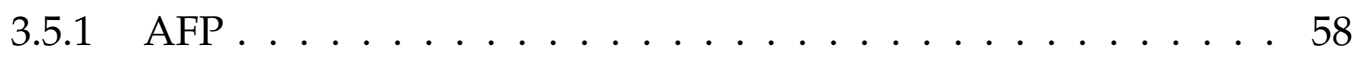




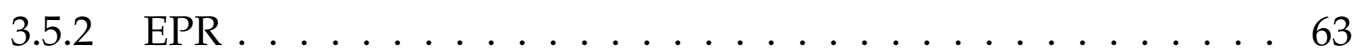

3.6 Optimization and Performance . . . . . . . . . . . . . . . . 69

3.7 Conclusions . . . . . . . . . . . . . . . . . . . 74

4 Steady State Free Precession $\quad 75$

$4.1 \quad$ RF Excitation . . . . . . . . . . . . . . . . . . . . . 77

4.2 Magnetization Evolution under Steady State Free Precession . . . . 78

4.3 Steady State Free Precession in Hyperpolarized Gas . . . . . . . . 84

4.4 Effects of Off-resonant Magnetization $\ldots \ldots \ldots \ldots$

4.5 Preliminary SSFP Measurements . . . . . . . . . . . . . . . . . 94

4.6 Diffusion-weighted SSFP with Hyperpolarized Magnetization . . . . 99

4.6.1 Symmetric Diffusion-Weighted SSFP . . . . . . . . . . . . 100

4.6.2 Asymmetric Diffusion-Weighted SSFP . . . . . . . . . . . . 105

4.6.3 Group of Three Diffusion-Weighted SSFP . . . . . . . . . . 118

4.6.4 Before/After Diffusion-Weighted SSFP ‥ . . . . . . . 127

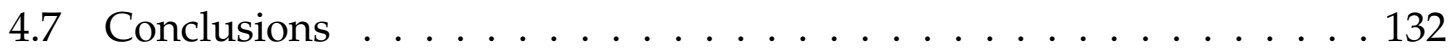

5 SSFP Measurements of Time-Dependent Diffusion 134

5.1 Time Dependence of the Restricted Diffusion Coefficient . . . . . . 135

5.2 General Experimental Methods . . . . . . . . . . . . . . . . . 140

5.3 Bead-Phantom Relaxation Measurements . . . . . . . . . . . . . 142

5.4 Pulse Sequence Tests . . . . . . . . . . . . . . . . . . . . . . . 144

5.5 Systematic Effects in Diffusion-weighted SSFP . . . . . . . . . . 151

5.6 Time-dependent Diffusion Measurements in Bead Phantoms . . . . . 159

5.7 Time-dependent Diffusion Measurements in Human Subjects . . . 164

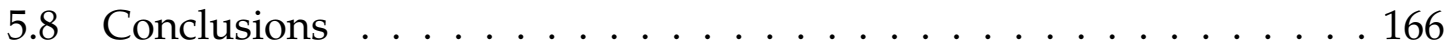


6 Hyperpolarized Gas MRI with Diffusion-weighted SSFP Pulse Sequences 168

6.1 SSFP Imaging Considerations . . . . . . . . . . . . . . . 169

6.2 Experimental Methods . . . . . . . . . . . . . . . . 170

6.3 Group of Three Imaging Test . . . . . . . . . . . . . . . . . 171

6.4 Before $/$ After SSFP DWI . . . . . . . . . . . . . . . . . . . 179

7 Conclusions $\quad 186$

A Hybrid Polarizer Limitations: Masing and the X-Factor 194

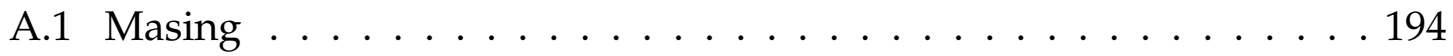

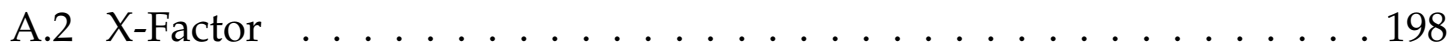

B Hybrid Polarizer Components $\quad 201$

B.1 Gas System Details . . . . . . . . . . . . . . . . . . . . . 201

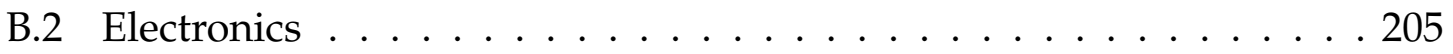

B.3 LabVIEW Programs . . . . . . . . . . . . . . . . . . 209

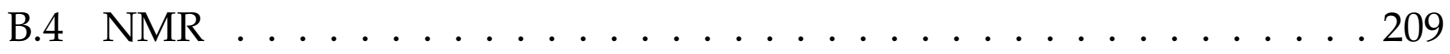

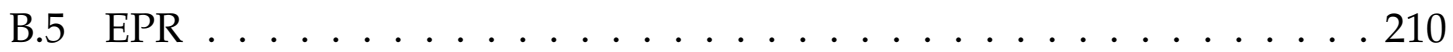

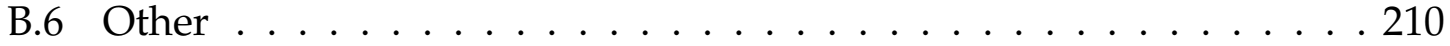

B.7 EPR Equations . . . . . . . . . . . . . . . . . . . 211

$\begin{array}{ll}\text { References } & 213\end{array}$ 


\section{Chapter 1}

\section{Introduction}

Magnetic Resonance Imaging (MRI) is a method of body imaging that was initially demonstrated by Paul Lauterbur in 1973 [1]; less than twenty years later it had become an essential and nearly ubiquitous medical technology. Magnetic resonance (MR) images are created by combining nuclear magnetic resonance (NMR) with magnetic field gradients which locally manipulate the precession frequencies of particles with spin in an external magnetic field. Lauterbur and Peter Mansfield shared a Nobel Prize in 2003 for their seminal work developing the basic techniques of MRI, and the field continues to inspire research and development today. The advancement of MRI techniques provides an opportunity to contribute significantly to many aspects of human biology, from the basic understanding of how the body functions to the diagnosis and treatment of disease.

The main application of MRI is imaging hydrogen nuclei $\left({ }^{1} \mathrm{H}\right)$, present in the body in large numbers in the forms of water and fat. The hydrogen nucleus consists of a single proton, which has an intrinsic spin which gives rise to a magnetic moment. The magnetic moment will tend to align and precess in the presence of external magnetic fields. By manipulating the size and direction of the external field through the use of field gradients and transverse resonant fields, images of 
various tissues in the body are made through a process discussed in Chapter 2.

The degree to which the nuclear spins of an ensemble are aligned with an external field is known as polarization. For a spin-1/2 particle like the ${ }^{1} \mathrm{H}$ nucleus, there are only two possible eigenstates in the presence of a magnetic field, aligned and anti-aligned. Polarization $P$ is defined as the asymmetry of the populations of the states:

$$
P=\frac{\left|N_{\uparrow}-N_{\downarrow}\right|}{\left|N_{\uparrow}+N_{\downarrow}\right|}
$$

where $N_{\uparrow}$ represents the number of particles in the aligned state and $N_{\downarrow}$ is the number of particles in the anti-aligned state.

At thermal equilibrium, the number of spins in each state $N_{i}$ at a given temperature $T$ is determined by the Boltzmann distribution. The energy of those states $E_{i}$ is related to the field strength $B$ and the gyromagnetic ratio of the particle $\gamma$. The thermal equilibrium polarization, $P_{\text {thermal }}$ for an ensemble of $N$ particles is calculated as follows:

$$
\begin{gathered}
\frac{N_{i}}{N}=\frac{g_{i} \frac{-E_{i}}{\kappa^{K_{B} T}}}{Z(T)}, \quad Z(T)=\sum_{i}, g_{i} e^{\frac{-E_{i}}{\kappa_{B} T}}, \quad N=\sum_{i} N_{i}, \quad E_{i}=\hbar \gamma B, \\
P_{\text {thermal }}=\tanh \frac{\hbar \gamma B}{2 \kappa_{B} T},
\end{gathered}
$$

where $g_{i}$ is the degeneracy of the state with energy $E_{i}, \hbar=1.05 \times 10^{-34} \mathrm{~J} \cdot \mathrm{s}$ is Planck's constant, and $\kappa_{B}=1.38 \times 10^{-23} \mathrm{~J} / \mathrm{K}$ is the Boltzmann constant. For hydrogen nuclei, $\gamma=2 \pi * 42.6 \mathrm{MHz} / \mathrm{T}$. In an external field of $B=1.5 \mathrm{~T}$ and body temperature of $T=37 \mathrm{C}, P_{\text {thermal }}=4.9 \times 10^{-6}$. This small net alignment results in a measurable signal due to the large ${ }^{1} \mathrm{H}$ density found in the body, $\sim 90,000$ mole $/ \mathrm{cm}^{3}$.

MRI is a useful tool for imaging a variety of areas in the body including skele- 
tal muscle, joints, and nearly every internal organ. Lung imaging however, has provided a challenge for MRI due to the sparsity of hydrogen nuclei in the lungs. The field of hyperpolarized gas imaging was created in 1994 to address this challenge [2]. The process involves imaging the airspaces in the lung after the subject inhales a noble gas that has been polarized to a level many orders of magnitude larger than the thermal equilibrium value. Helium-3 and xenon-129 both have a nuclear spin of $1 / 2$, and are the most common gases used in hyperpolarized gas MR. In order to generate a measurable signal, the gas must be prepared with a polarization approaching unity $\left(P_{\text {gas }} \rightarrow 1\right)$ to compensate for the reduced particle density of the gas $\left(\sim 50 \mathrm{~mole} / \mathrm{cm}^{3}\right)$. The process of producing helium-3 with such a large polarization is the subject of Chapter 3. Hyperpolarized gas MRI provides a unique view of the lung, offering both structural and functional information. The images produced with this technique have been shown to be useful tools in assessing various forms of lung disease such as asthma [3, 4], cystic fibrosis [5], and chronic obstructive pulmonary disease (COPD) [6, 7].

Prior to the development of hyperpolarized gas MRI the primary tools for studying lung disease were pulmonary function tests, X-ray computed tomography (CT), and lung histology. Each of these methods has drawbacks which limit their ability to characterize disease processes. The most common pulmonary function test is spirometry, the process of characterizing ventilatory lung function based on air volume and flow during inhalation and exhalation. A spirometry test provides no regional information, and is generally not sensitive to small changes in lung structure. CT scans provide detailed, high-resolution images of the lung tissue, but generally provide structural rather than functional information. The radiation dose associated with each CT scan also limits the use of CT in long-term studies. Histology is the process of taking thin slices of tissue samples and exam- 
ining them on microscopic slides. These slides can be evaluated using stereological methods for quantitative parameters such the mean chord length, which in the lung can be converted to the surface-to-volume ratio of the alveoli. The major drawback of using lung histology to characterize lung disease is the invasive nature of sample collection. Histology is a common tool when studying animal models of lung disease, but is significantly less useful in human research.

Hyperpolarized gas MRI does not share any of these drawbacks, and has the potential to become a standard measure of lung disease characterization. Lung imaging with hyperpolarized gas MRI can provide regional information about lung function and structure, with a great potential for use in long-term research studies. However the gases and technologies required for imaging are not currently well disseminated or optimized for wide-spread use. Achieving the goal of establishing hyperpolarized gas MRI as a key tool in lung disease assessment depends on further optimization and development, which are the underlying goals of this work.

An area where the benefits of hyperpolarized gas MR can contribute significantly is in diagnosing and monitoring chronic obstructive pulmonary disease (COPD). COPD is responsible for 2-3 million deaths worldwide each year [8], and is projected to be the third leading cause of death in the US by 2020 [9]. Patients with COPD are primarily smokers, although not all smokers develop the disease. COPD has two main components which can present jointly or independently, airflow limitation (chronic bronchitis) and tissue destruction (emphysema). The MRI techniques developed in this thesis are primarily aimed at studying emphysema.

Emphysema progresses by destroying alveolar walls, which change both the structure and performance of the lungs. Healthy lungs consist of many alveolar sacs of about $\sim 200-300 \mu \mathrm{m}$ diameter [10]. As the disease progresses, the walls be- 
tween the alveoli are destroyed which reduces the surface area of lung tissue and decreases the capacity for gas exchange. Currently, emphysema is categorized by a pulmonary function test [11], but this gives no regional information about the disease. There is usually considerable tissue destruction before lung function suffers to the point of diagnosis. CT imaging can provide regional information about destruction through a reduction in tissue density, however the radiation dose required means that it is not a practical alternative to pulmonary function tests for long-term studies of emphysema.

There is currently no cure for emphysema, only treatments to manage the symptoms. Future drug treatment developments depend on the ability to assess the effectiveness of treatments. Hyperpolarized gas MRI has the potential to provide a tool for frequently assessing disease characteristics without contributing ill-effects (such as radiation dose) from the measurement technique.

While lung airspace images produced by hyperpolarized gas MRI (often referred to as ventilation images) are useful for characterizing many aspects of lung disease, they are unable to reach the spatial resolution required to visualize the effects of emphysema on alveolar walls. The minimum resolution in a ventilation MR image is limited by several factors, such as the size of field gradients that can be generated by the MRI scanner and gas diffusion during the image acquisition. Because the gas particles are continually moving while being imaged, they cannot be localized to an region smaller than the distance they have traveled. The resolution limit due to gas diffusion over a typical hyperpolarized gas MR acquisition is $\sim 1 \mathrm{~mm}$. This distance is several times larger than an alveolor diameter, and many times larger than the thickness of the alveolor walls themselves ( $\sim 10 \mu \mathrm{m}[12])$.

The diffusion process describes how particles of a gas or liquid move through a fluid over time. Particles undergo Brownian motion in which they randomly 


\begin{tabular}{|c|c|c|}
\hline Species & $D_{0}$ (pure) & $D_{0}$ (in air) \\
\hline${ }^{3} \mathrm{He}$ & $1.9 \mathrm{~cm}^{2} / \mathrm{s}$ & $0.88 \mathrm{~cm}^{2} / \mathrm{s}$ \\
\hline${ }^{129} \mathrm{Xe}$ & $0.06 \mathrm{~cm}^{2} / \mathrm{s}$ & $0.14 \mathrm{~cm}^{2} / \mathrm{s}$ \\
\hline $\mathrm{H}_{2} \mathrm{O}$ & $2.3 * 10^{-5} \mathrm{~cm}^{2} / \mathrm{s}$ & - \\
\hline
\end{tabular}

Table 1.1: Free diffusion coefficients of common MR nuclei.

explore the medium. Diffusivity $D(t)$ is defined as the mean-squared displacement of a particle over a given period of time $t$ :

$$
\begin{array}{r}
D(t) \equiv \frac{\left\langle[\mathbf{r}(t)-\mathbf{r}(0)]^{2}\right\rangle}{2 d t}, \\
d=1,2,3 \text { for 1D, 2D, 3D diffusion, }
\end{array}
$$

where $\mathbf{r}(t)$ is the particle location. For particles diffusing in free space, $D(t)=D_{0}$, the free diffusion coefficient. $D_{0}$ is an intrinsic time-independent quantity that is characteristic of specific partial pressures and mixtures of gases. In Table 1.1, the free diffusivities are listed for the two most common hyperpolarized imaging gases helium-3 and xenon-129, as well as for water. The gas particles diffuse much more quickly than the liquid particles.

For particles diffusing in the empty spaces of a porous medium, the diffusive motion is restricted not only by other freely diffusing particles, but also by the walls of the pore space. In this situation, referred to as restricted diffusion, the diffusivity is time-dependent, and decreases as a function of measurement time $t$.

Diffusion-weighted MRI is a technique that exploits particle movement during the imaging acquisition to produce image contrast. As will be explained in Chapter 2, the contrast can be manipulated to extract a measurement of diffusivity. When applied to inhaled hyperpolarized gases, diffusion-weighted MRI can be used to reveal details about the area in which the particles are diffusing (the alveoli), providing structural information below the pixel resolution limit of the raw 
images. When diffusivity is measured in the body by MRI, the values are referred to as apparent diffusion coefficients, or ADCs. This distinction is made because $\mathrm{ADC}$ values generally have some residual dependence on measurement parameters, whereas the true diffusivity is an intrinsic property of the diffusing particles and the containing space.

The length scale being probed by a diffusion measurement is given by the measurement time and the diffusivity, obtained by rearranging Equation 1.4:

$$
\Delta r=\sqrt{2 d D t} .
$$

When diffusing particles are placed in a restrictive space, the mean-squared displacement no longer increases in proportion to measurement time, which results in diffusivity $D(t)$ that is lower than the free diffusion coefficient $D_{0}$ and decreases with measurement time. The degree to which the particles are confined will determine how much lower than $D_{0}$ the diffusivity is and how quickly it decreases with time. However if the diffusivity of confined particles is measured at short diffusion times, i.e. before most particles have traveled far enough to contact a wall, then the value measured would be very close to the free diffusion coefficient $\left(D(0)=D_{0}\right)$. This dependency on measurement time is essential to our measurement technique, which will be detailed in Chapters 2 and 4 .

There are many examples in the literature of hyperpolarized gas diffusionweighted MRI in which the ADC is measured in the lung using diffusion times of $t \sim 1-2 \mathrm{~ms}$ [13-18]. Assuming three-dimensional diffusion and a diffusivity in healthy lungs of $D \sim 0.27 \mathrm{~cm}^{2} / \mathrm{s}$ [18], we can use Equation 1.5 to calculate the length scale that diffusivity measurements at $t=1-2 \mathrm{~ms}$ are sensitive to:

$$
\Delta r=\sqrt{2 d D t}=400-570 \mu \mathrm{m},
$$


which is larger than the alveolar size of $200-300 \mu \mathrm{m}$.

There is significant value in developing a diffusion-weighted MR technique that is sensitive to length scales closer to alveolar size, as it would potentially be more sensitive to the early stages of lung tissue destruction caused by emphysema. The goal of this thesis is to develop a method of making hyperpolarized gas diffusion MR measurements at shorter diffusion times such that the length scale probed approaches the alveolar length scale. The methods we developed to make diffusion-weighted measurements at short diffusion times is described in Chapter 4. The key feature of our basic strategy is an MR pulse sequence technique known as steady state free precession (SSFP). SSFP is a class of pulse sequences that uses the rapid application of RF pulses to generate a large coherent MR signal.

A diffusion-weighted SSFP MR pulse sequence was used to make global (nonimaging) ADC measurements of hyperpolarized helium-3 in structured glass cells and human subjects, and these results are presented in Chapter 5. In Chapter 6, an imaging version of the pulse sequence was used to create ADC maps in human subjects at a diffusion time of $t=500 \mu \mathrm{s}$, reducing our length scale sensitivity to $\Delta r \approx 280 \mu \mathrm{m}$. 


\section{Chapter 2}

\section{Magnetic Resonance Imaging}

Magnetic resonance imaging (MRI) is a technique which combines nuclear magnetic resonance (NMR) with magnetic field gradients to produce spin-density images [1]. When imaging the human body, the most common object of MRI is the hydrogen nucleus $\left({ }^{1} H\right)$ which is primarily present in the body in the forms of water or fat. The high density of hydrogen nuclei $\left(\sim 90,000 \mathrm{~mole} / \mathrm{cm}^{3}\right)$ creates a large NMR signal from the thermal equilibrium polarization generated by the MRI scanner's main magnetic field, which for the work in this thesis was primarily at $B_{0}=1.5 \mathrm{~T}$. Areas in the body that have a lower density of water or fat, such as the lungs, are difficult to image with MRI. Hyperpolarized gas MR is a field which has emerged to address this problem by filling the lungs with a mixture of air and a pre-polarized noble gas (typically helium-3 or xenon-129) which can be imaged.

In this chapter I describe how magnetic resonance images are made, and how they can be used to study lung disease such as emphysema. First, I discuss how particles with spin behave in the presence of a static, uniform magnetic field. Section 2.2 outlines how the application of resonant magnetic fields leads to NMR, followed by how the addition of magnetic field gradients leads to imaging in Section 2.3. In Section 2.4 I discuss the changes required for adapting MRI from the de- 
tection of thermally-polarized hydrogen nuclei to the detection of hyperpolarized gases in the lung. The method of incorporating diffusion sensitization into MR is described in Section 2.5.

\subsection{Spins in a Magnetic Field}

Hydrogen nuclei and other targets of NMR have a non-zero spin $\vec{S}$ which causes them to have an intrinsic magnetic moment,

$$
\vec{\mu}=\gamma \vec{S}
$$

where $\gamma$ is the particle's gyromagnetic ratio. In the presence of an external magnetic field, the classical description of the spin's behavior is that it will tend to align with the direction of the field and precess around it when not perfectly aligned (Figure 2.1a).

In the quantum description, the quantization of spin results in a set of eigenstates $m_{S}$ which have discrete directions of alignment. In the case of $\operatorname{spin} S=\frac{1}{2}$ particles like the hydrogen nucleus, there are only two eigenstates of the spin magnetic moment, $m_{S}= \pm \frac{1}{2}$ (Figure 2.1b).

The quantum expectation value of the magnetic moment is analogous to the classical magnetization and is a superposition of the eigenstates. The expectation value is given by:

$$
\langle\Psi|\vec{\mu}(t)| \Psi\rangle
$$

where $|\Psi\rangle$ is the wavefunction of the spin $1 / 2$ system,

$$
\mathbf{\Psi}(\mathbf{t})=\sum_{m= \pm 1 / 2} c_{m} \mathbf{\Psi}_{m} e^{-\frac{i}{\hbar} E_{m} t}
$$




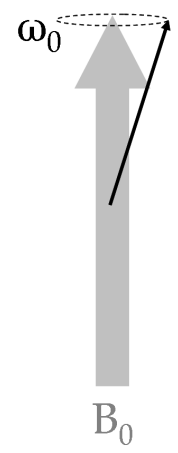

(a) Classical magnetic particle

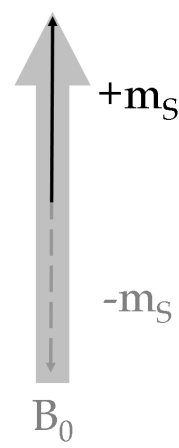

(b) Quantum spin eigenstates

Figure 2.1: A spin in a magnetic field $B_{0}$.

The $\Psi_{m}$ are the vector eigenstates, and the complex coefficients $c_{m}$ are determined by initial conditions,

$$
c_{ \pm 1 / 2}=a_{ \pm} e^{i \alpha_{ \pm}}
$$

The coefficients satisfy the normalization condition:

$$
\sum_{m}\left|c_{m}\right|^{2}=1
$$

In the presence of an external field $\vec{B}=B_{0} \hat{z}$, the energy of each state is given by:

$$
E=-\vec{\mu} \cdot \vec{B}=-\hbar \gamma m_{z} B_{0}
$$

where $\hbar$ is Planck's constant and $m_{z}= \pm \frac{1}{2}$ is the component of spin along the $\hat{z}$-direction. The energy required to transition between the two states is:

$$
E_{1}-E_{2}=\Delta E= \pm \hbar \gamma B_{0}= \pm \hbar \omega_{0}
$$

where $\omega_{0}$ is known as the Larmor frequency, which is the precession frequency of the classical magnetization. 
The correspondence between the classical and the quantum mechanical pictures can be further appreciated by considering the component of the magnetic moment that is transverse to the applied field. When determining the expectation value of a spin $1 / 2$ system in the basis of the eigenstates of $S_{z}$, the spin operator $\vec{S}$ can be mathematically represented by the Pauli spin matrices, $\vec{S}=$ $\frac{\hbar}{2}\left(\sigma_{x} \hat{x}+\sigma_{y} \hat{y}+\sigma_{z} \hat{z}\right):$

$$
\sigma_{x}=\left(\begin{array}{ll}
0 & 1 \\
1 & 0
\end{array}\right), \quad \sigma_{y}=\left(\begin{array}{cc}
0 & -i \\
i & 0
\end{array}\right), \quad \sigma_{z}=\left(\begin{array}{cc}
1 & 0 \\
0 & -1
\end{array}\right)
$$

The expectation value of $\mu_{x}(t)$ is given by:

$$
\left\langle\mathbf{\Psi}\left|\mu_{x}(t)\right| \mathbf{\Psi}\right\rangle=\sum_{m} \sum_{m^{\prime}} c_{m^{\prime}}^{*} \mathbf{\Psi}_{\mathbf{m}^{\prime}}^{\dagger} \gamma \frac{\hbar}{2} \sigma_{x} c_{m} \mathbf{\Psi}_{\mathbf{m}} e^{\frac{i}{\hbar}\left(E_{m^{\prime}}-E_{m}\right) t}
$$

Only the cross-terms survive due to the orthonormality of the eigenstates.

$$
\left\langle\mu_{x}(t)\right\rangle=\frac{\hbar \gamma}{2}\left(c_{-1 / 2}^{*} c_{1 / 2} e^{i \omega_{0} t}+c_{1 / 2}^{*} c_{-1 / 2} e^{-i \omega_{0} t}\right)
$$

Substituting in the complex representation of the $c_{m}$ :

$$
\begin{gathered}
\left\langle\mu_{x}(t)\right\rangle=\frac{\hbar \gamma}{2}\left(a_{-} a_{+} e^{-i\left(\alpha_{-}-\alpha_{+}-\omega_{0} t\right)}+a_{+} a_{-} e^{i\left(\alpha_{-}-\alpha_{+}-\omega_{0} t\right)}\right) \\
=\frac{\hbar \gamma}{2} a_{-} a_{+}\left(e^{-i\left(\alpha_{-}-\alpha_{+}-\omega_{0} t\right)} e^{i\left(\alpha_{-}-\alpha_{+}-\omega_{0} t\right)}\right) \\
=\hbar \gamma a_{-} a_{+} \cos \left(\alpha_{-}-\alpha_{+}-\omega_{0} t\right) .
\end{gathered}
$$

From the normalization condition of the $c_{m}$, we have:

$$
1=\left|a_{+}^{2}+a_{-}^{2}\right|
$$


This condition can be satisfied by:

$$
a_{+}=\cos (\Theta / 2) \quad \alpha_{-}=\sin (\Theta / 2),
$$

where the significance of the angle $\Theta$ will be explained later. We substitute the $a_{ \pm}$ into the solution for $\left\langle\mu_{x}(t)\right\rangle$,

$$
\begin{gathered}
\left\langle\mu_{x}(t)\right\rangle=\hbar \gamma \cos (\Theta / 2) \sin (\Theta / 2) \cos \left(\alpha_{-}-\alpha_{+}-\omega_{0} t\right) \\
=\frac{\hbar}{2} \gamma \sin \Theta \cos \left(\alpha_{-}-\alpha_{+}-\omega_{0} t\right) .
\end{gathered}
$$

Substituting in $\phi_{0} \equiv \alpha_{-}-\alpha_{+}$into Equation 2.17 we have:

$$
\left\langle\mu_{x}(t)\right\rangle=\frac{\hbar}{2} \gamma \sin \Theta \cos \left(\phi_{0}-\omega_{0} t\right) .
$$

The solutions for $\left\langle\mu_{y}(t)\right\rangle$ and $\left\langle\mu_{z}(t)\right\rangle$ are:

$$
\begin{gathered}
\left\langle\mu_{y}(t)\right\rangle=\frac{\hbar}{2} \gamma \sin \Theta \sin \left(\phi_{0}-\omega_{0} t\right), \\
\left\langle\mu_{z}(t)\right\rangle=\frac{\gamma \hbar}{2} \cos \Theta .
\end{gathered}
$$

The expectation value of the magnetic moment $\langle\Psi|\vec{\mu}(t)| \Psi\rangle$ in the presence of a constant external magnetic field in $\hat{z}$-direction is a vector tipped by an angle $\Theta$ which precesses around the field at the frequency $\omega_{0}$. $\Theta$ is determined by the relative weighting of the two eigenstates, and $\phi_{0}$ represents an initial angle in the $\hat{x}-\hat{y}$ plane. The solution for the quantum expectation value of the magnetic moment is consistent with the classical description of a spin precessing around the external field at $\omega_{0}$. In the classical picture, the angle $\Theta$ represents the angle between the spin vector and the external field. 
Larmor precession frequencies for hydrogen nuclei and the two noble gases commonly used for hyperpolarized gas MRI can be found in Table 2.1 for MRI scanner main field values of $1.5 \mathrm{~T}$ and $3 \mathrm{~T}$.

\begin{tabular}{|c|c|c|c|}
\hline Nuclei & $\gamma / 2 \pi$ & $f_{0}=\frac{\omega_{0}}{2 \pi}(1.5 T)$ & $f_{0}=\frac{\omega_{0}}{2 \pi}(3 T)$ \\
\hline${ }^{1} \mathrm{H}$ & $42.576 \mathrm{MHz} / \mathrm{T}$ & $63.67 \mathrm{MHz}$ & $123.19 \mathrm{MHz}$ \\
\hline${ }^{3} \mathrm{He}$ & $-32.43 \mathrm{MHz} / \mathrm{T}$ & $48.50 \mathrm{MHz}$ & $93.83 \mathrm{MHz}$ \\
\hline${ }^{129} \mathrm{Xe}$ & $-11.78 \mathrm{MHz} / \mathrm{T}$ & $17.62 \mathrm{MHz}$ & $34.08 \mathrm{MHz}$ \\
\hline
\end{tabular}

Table 2.1: Resonant frequencies for MR nuclei.

\subsection{Nuclear Magnetic Resonance}

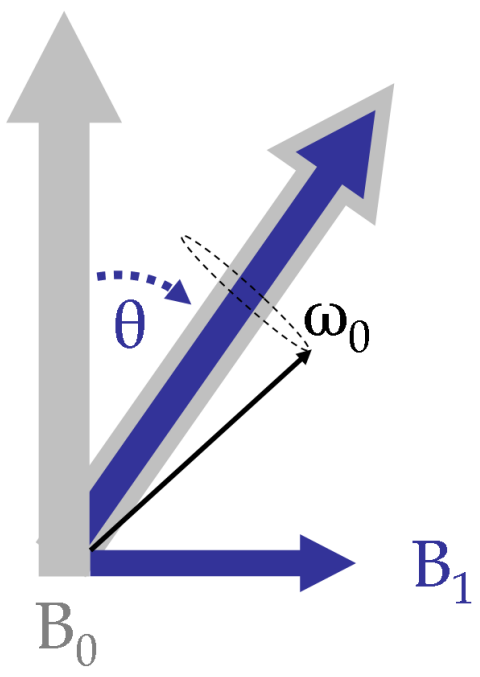

(a) Application of transverse field.

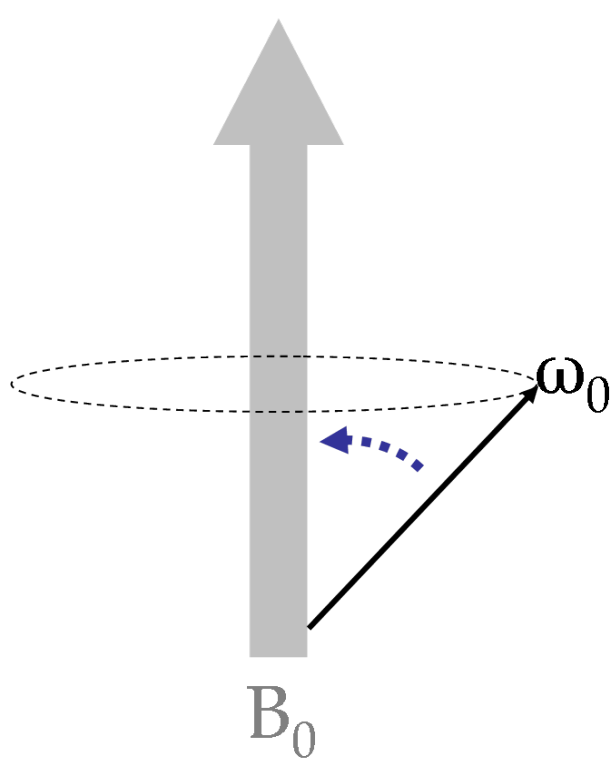

(b) Decay toward equilibrium.

Figure 2.2: Nuclear magnetic resonance: (a) A transverse field $B_{1}\left(\omega_{0}, t\right)$ induces precession around the effective field, the combination of $B_{0}$ and $B_{1}$. (b) After $B_{1}$ is removed, the spin continues to precess as it decays back to the equilibrium orientation.

In nuclear magnetic resonance, the alignment direction of the magnetic moment with respect to the main magnetic field can be altered by the application 
of an additional resonant frequency (RF) magnetic field. In the classical picture shown in Figure 2.2, a short pulse of perpendicular magnetic field $B_{1}\left(\omega_{0}, t\right)$ is applied. The spin will precess about the new effective field, the combination of the static $B_{0}$ and the resonant $B_{1}$ and thus rotate the spin away from the main external field into the transverse plane by an angle $\theta$, referred to as the flip angle. The duration and amplitude of the transverse RF field determine $\theta$.

In the quantum description, the resonant field drives transitions between spin eigenstates in the particles and has the net effect of altering the relative weighting of the states, thereby changing the expectation value of the magnetic moment. In the presence of a transverse RF field, $\vec{B}_{\text {Trans }}(t)=B_{1}\left(\cos \omega_{0} t \hat{x}+\sin \omega_{0} t \hat{y}\right)$, the spin Hamiltonian becomes:

$$
H(t)=-\vec{\mu} \cdot \vec{B}_{\text {Total }}(t)=-\frac{\gamma \hbar}{2}\left(B_{1} \sigma_{x} \cos \omega_{0} t+B_{1} \sigma_{y} \sin \omega_{0} t+B_{0} \sigma_{z}\right) .
$$

The wavefunction for this system is given by:

$$
\mathbf{\Psi}(\mathbf{t})=\sum_{m= \pm 1 / 2} c_{m}(t) \Psi_{m} e^{-\frac{i}{\hbar} E_{m} t}
$$

The wave variables $c_{m}(t)$ satisfy the Schrödinger equation:

$$
\frac{d c_{-1 / 2}}{d t}=\frac{i}{2} \omega_{0} c_{+1 / 2}
$$

with solutions as follows:

$$
\begin{array}{r}
c_{+1 / 2}=a \sin \frac{\omega_{0} t}{2}+b \cos \frac{\omega_{0} t}{2} \\
c_{-1 / 2}=-i b \sin \frac{\omega_{0} t}{2}+i a \cos \frac{\omega_{0} t}{2},
\end{array}
$$


where $a$ and $b$ are arbitrary constants. The normalization condition of:

$$
1=\left(|a|^{2}+|b|^{2}\right)
$$

allows $a$ and $b$ to be written as:

$$
a=\cos (\Theta / 2) e^{-i \alpha}, \quad b=\sin (\Theta / 2) e^{-i \phi}
$$

where $\Theta, \alpha$, and $\phi$ are determined by initial conditions.

The eigenstates for the Hamiltonian are used to calculate a new expectation value for the magnetic moment $\langle\vec{\mu}(t)\rangle$. The behavior of the spin in response to the transverse field can be derived more easily in a reference frame rotating about the $\hat{z}$ axis at the resonant frequency $\omega_{0}$ pictured in Figure 2.3. The rotating frame of reference is often denoted by $\hat{x}^{\prime}-\hat{y}^{\prime}$. In this frame, the magnetic moment and transverse RF field are constant in time. The spin matrices translate between the rotating frame and the laboratory frame as follows:

$$
\sigma_{x^{\prime}}=\cos \omega_{0} t \sigma_{x}-\sin \omega_{0} t \sigma_{y,} \quad \sigma_{y^{\prime}}=\sin \omega_{0} t \sigma_{x}+\cos \omega_{0} t \sigma_{y}
$$

We can solve for the new expectation value of $\mu_{x^{\prime}}$,

$$
\left\langle\Psi\left|\mu_{x}^{\prime}(t)\right| \mathbf{\Psi}\right\rangle=\sum_{m} \sum_{m^{\prime}} c_{m^{\prime}}^{*} \mathbf{\Psi}_{\mathbf{m}^{\prime}}^{\dagger} \gamma \frac{\hbar}{2} \sigma_{x^{\prime}} c_{m} \mathbf{\Psi}_{\mathbf{m}} e^{\frac{i}{\hbar}\left(E_{m^{\prime}}-E_{m}\right) t}
$$

Substituting in the values of the $c_{m}$ from Equations 2.25 and 2.27 we have:

$$
\left\langle\mu_{x^{\prime}}(t)\right\rangle=\frac{\gamma \hbar}{2} \sin \Theta \cos (\alpha-\phi-\pi / 2)
$$

where $\Theta$ can be thought of as the initial polar angle and $\Phi \equiv(\alpha-\phi-\pi / 2)$ the 


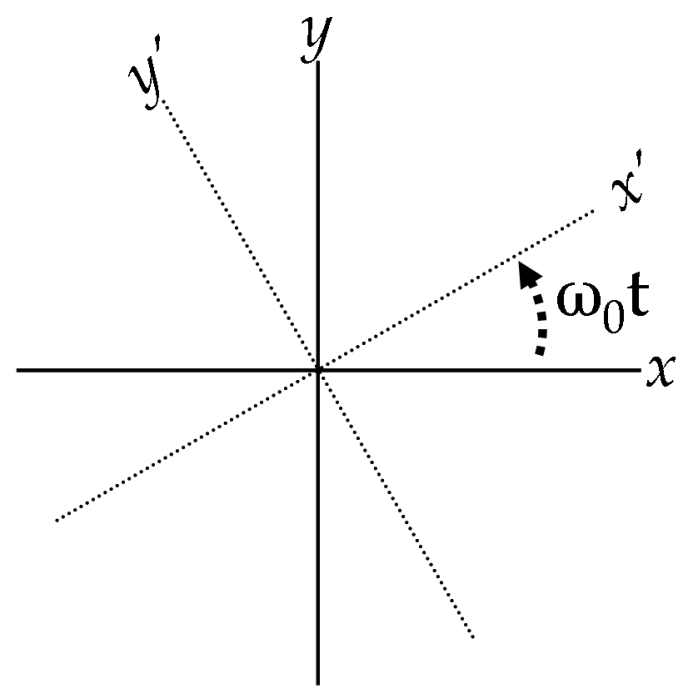

Figure 2.3: A reference frame which is rotating at the resonant frequency $\omega_{0}$ with respect to the laboratory.

initial azimuthal angle. The values of $\left\langle\mu_{y^{\prime}}(t)\right\rangle$ and $\left\langle\mu_{z}(t)\right\rangle$ are as follows:

$$
\begin{aligned}
& \left\langle\mu_{y^{\prime}}(t)\right\rangle=\frac{\gamma \hbar}{2} \cos \Theta \sin \omega_{0} t+\sin \Theta \sin \Phi \cos \omega_{0} t \\
& \left\langle\mu_{z}(t)\right\rangle=\frac{\gamma \hbar}{2} \cos \Theta \cos \omega_{0} t-\sin \Theta \sin \Phi \sin \omega_{0} t
\end{aligned}
$$

In the presence of a transverse field, the magnetic moment expectation value has a constant component in the $\hat{x}^{\prime}$ direction, and is precessing about that axis at $\omega_{0}$. The classical picture of a spin tipped by the angle $\theta=\omega_{0} t$ is preserved in the quantum formalism. In practice the flip angle $\theta$ is specified for a given application, and the RF pulse strength and duration are calculated based on the particular system being used for the experiment.

The connection between the quantum mechanical expectation value of the magnetic moment and the classical magnetization vector allows for consideration of the latter to be generally sufficient to describe the behavior of the system. For the remainder of the discussion, I will refer to a spin's (or collection of spins') magne- 
tization $\vec{M}$, rather than the quantum magnetic moment. For spin $1 / 2$ particles, the two quantities are related by:

$$
M_{i}(t)=N P \mu_{i}(t)
$$

where $N$ is the number of particles, and $P$ is the ensemble polarization. The signal $S(t)$ measured by NMR is proportional to the magnetization in the transverse plane:

$$
S(t) \propto M_{T}(t)=M_{x}(t)+i M_{y}(t)
$$

After the RF pulse ends, the magnetization precesses around the main field as it decays back to the equilibrium alignment. This decay process is characterized by two time constants $T_{1}$ and $T_{2}$, listed in Table 2.2 for hydrogen nuclei in various tissues as well as helium-3 and xenon-129 [19-22]. The magnetization in the $\hat{z}$ direction $M_{z}(t)$ relaxes towards the Boltzmann thermal equilibrium value $M_{0}$ with a time constant known as $T_{1}$ :

$$
\frac{\partial M_{z}}{\partial t}=\frac{M_{0}-M_{z}}{T_{1}} .
$$

The longitudinal magnetization is given by:

$$
M_{z}(t)=M_{z}(0) e^{-\frac{t}{T_{1}}}+M_{0}\left(1-e^{-\frac{t}{T_{1}}}\right)
$$

The precession and decay are measured in the transverse plane, with the signal referred to here as a free induction decay (FID). In the $\hat{x}-\hat{y}$ plane, the magnetization components $M_{x}(t)$ and $M_{y}(t)$ relax toward equilibrium (which in this plane is zero) with a time constant known as $T_{2}$. However given an ensemble of spins, the detected signal goes to zero faster than the individual spins' transverse mag- 
netizations. This signal loss is primarily due to random small magnetic field inhomogeneities, $\vec{B}(\vec{r})=\left(B_{0} \pm \epsilon(\vec{r})\right) \hat{z}$, which cause the spins to accumulate phase differences as they precess at slightly different frequencies. The phase differences result in a diminishing vector sum, which goes to zero faster than $T_{2}$ as the spins "fan out" as shown in Figure 2.4a. Spins which precess at $\omega_{0}=\gamma B_{0}$ are referred to as on-resonance, whereas spins with either a higher or lower precession frequency are known as off-resonant spins.

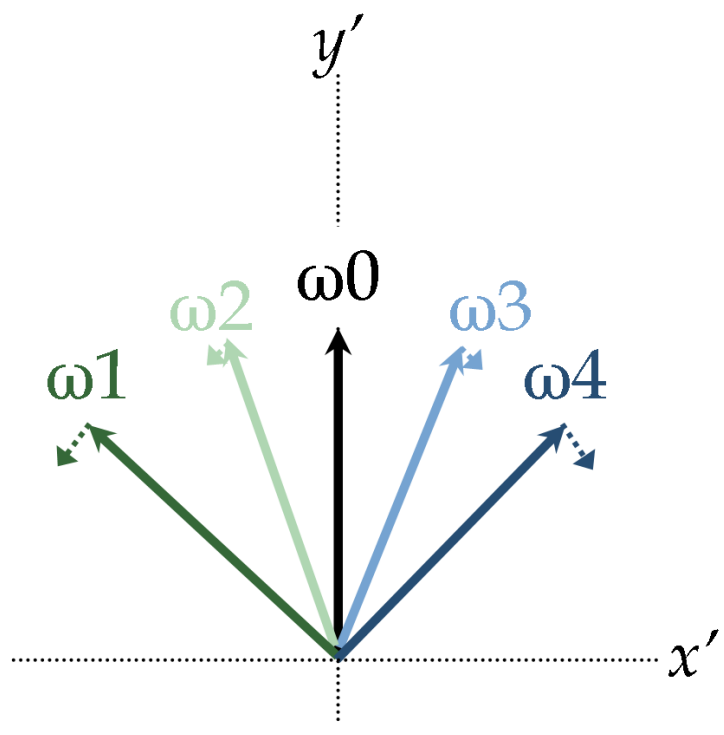

(a) Phase Accumulation

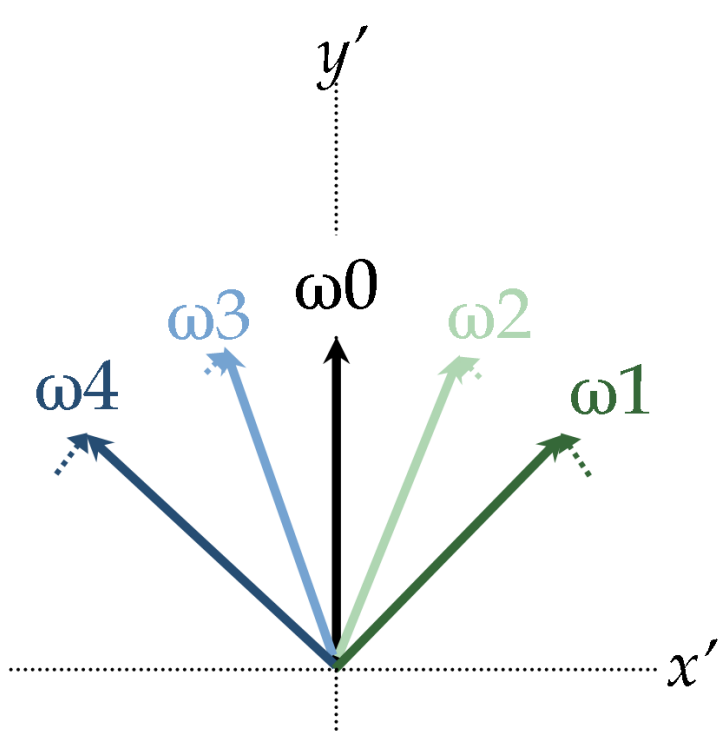

(b) Phase Reversal

Figure 2.4: Signal evolution due to magnetic field inhomogeneities. In the rotating frame, on-resonance spins are stationary, while spins with higher (lower) resonant frequencies precess counter-clockwise (clockwise). (a) Magnetic field inhomogeneities result in a variety of precession frequencies in the sample. The net signal is a coherent sum of all of the transverse magnetization vectors, which is lower than the sum of the individual magnitudes. (b) The spins from (a) are shown immediately after an RF pulse which has induced $180^{\circ}$ rotation about the $\hat{y}^{\prime}$ axis. The accumulated phase of each spin has been reversed, and they continue to evolve with the same frequency offset.

It is a convenient simplification to consider the net transverse magnetization evolution as an exponential decay with a time constant of $T_{2}^{*}$, but the true temporal behavior can be much more complicated [23]. Under the assumption of exponen- 
tial decay, we can solve for $M_{x}(t)$ and $M_{y}(t)$ using:

$$
\begin{aligned}
& \frac{\partial M_{x}}{\partial t}=\omega_{0} M_{y}-\frac{M_{x}}{T_{2}^{*}}, \\
& \frac{\partial M_{y}}{\partial t}=\omega_{0} M_{x}-\frac{M_{y}}{T_{2}^{*}} .
\end{aligned}
$$

The $\hat{x}$ and $\hat{y}$ components of $\vec{M}(t)$ are:

$$
\begin{aligned}
& M_{x}(t)=e^{-\frac{t}{T_{2}^{*}}}\left(M_{x}(0) \cos \omega_{0} t+M_{y}(0) \sin \omega_{0} t\right), \\
& M_{y}(t)=e^{-\frac{t}{T_{2}^{*}}}\left(M_{y}(0) \cos \omega_{0} t-M_{x}(0) \sin \omega_{0} t\right),
\end{aligned}
$$

and the total transverse component is given by:

$$
\begin{gathered}
M_{T}(t)=M_{x}(t)+i M_{y}(t), \\
M_{T}(t)=M_{T}(0) e^{-\left(i \omega_{0}+\frac{1}{T_{2}^{*}}\right) t} .
\end{gathered}
$$

If the magnetic field inhomogeneities which cause dephasing are constant in time, the net signal decay due to loss of coherence can be reversed by applying an additional RF pulse of flip angle $180^{\circ}$ and waiting an appropriate amount of time. Figure $2.4 \mathrm{~b}$ shows the state of the spins from Figure 2.4a immediately after a $180^{\circ}$ pulse about the $\hat{y}^{\prime}$ axis. The phases of the spins are reversed, but they continue to evolve with their previous precession frequencies. The phase accumulation "rewinds", and the net signal recovers to the level that each spin has lost due to the $T_{2}$ time constant. Figure 2.5 graphs the RF excitation on the top graph, and the measured transverse signal on the bottom. The oscillating signal measured in the lab frame (blue dotted line) and the demodulated signal corresponding to the rotating frame magnetization (blue solid line) demonstrate the signal reduction 
due to off-resonant spins. At $t=T E / 2$ the refocusing RF pulse is applied, and the net signal begins to increase. The red line shows the $T_{2}$-decay curve, which the measured signal reaches at $t=T E$, the so-called echo time. The signal peak at $t=T E$ is referred to as a spin echo [24]. The time constant of the reversible portion of the decay is often referred to as $T_{2}{ }^{\prime}$. The relation between the transverse time constants is:

$$
\frac{1}{T_{2}^{*}}=\frac{1}{T_{2}}+\frac{1}{T_{2}^{\prime}} .
$$

Spin-echo pulse sequences with $180^{\circ}$ refocusing-RF pulses are not commonly used with hyperpolarized media, therefore the values listed in Table 2.2 for helium and xenon are measurements of $T_{2}^{*}$, not $T_{2}$. However, RF pulses with flip angles less than $180^{\circ}$ can generate partial refocusing effects. The partial refocusing of offresonant phase accumulation plays an important role in steady state free precession (SSFP) signal dynamics which are discussed in Chapter 4.

\begin{tabular}{|c|c|c|c|c|}
\hline Tissue & $T_{1}(1.5 \mathrm{~T})$ & $T_{2}(1.5 \mathrm{~T})$ & $T_{1}(3 \mathrm{~T})$ & $T_{2}(3 \mathrm{~T})$ \\
\hline${ }^{1} \mathrm{H}$ in muscle & $1000 \mathrm{~ms}$ & $45 \mathrm{~ms}$ & $1400 \mathrm{~ms}$ & $50 \mathrm{~ms}$ \\
\hline Fat & $290 \mathrm{~ms}$ & $160 \mathrm{~ms}$ & $360 \mathrm{~ms}$ & $130 \mathrm{~ms}$ \\
\hline Tissue & $T_{1}(1.5 \mathrm{~T})$ & $T_{2}^{*}(1.5 \mathrm{~T})$ & $T_{1}(3 \mathrm{~T})$ & $T_{2}^{*}(3 \mathrm{~T})$ \\
\hline${ }^{3} \mathrm{He}$ in lung & $20 \mathrm{~s}$ & $28 \mathrm{~ms}$ & - & $14 \mathrm{~ms}$ \\
\hline${ }^{129} \mathrm{Xe}$ in lung & $30 \mathrm{~s}$ & $50 \mathrm{~ms}$ & - & $27 \mathrm{~ms}$ \\
\hline
\end{tabular}

Table 2.2: Time constants for common MR tissues.

We have shown that the presence of random magnetic field inhomogeneities can result in a reduction of signal coherence in the NMR measurement. However, systematic magnetic field variations such as a linear field gradient would lead to systematic precession frequency differences. The next section details how applied magnetic field gradients can be used to localize spins through the resulting interference patterns and produce magnetic resonance images. 


\section{Spin Echo Technique}
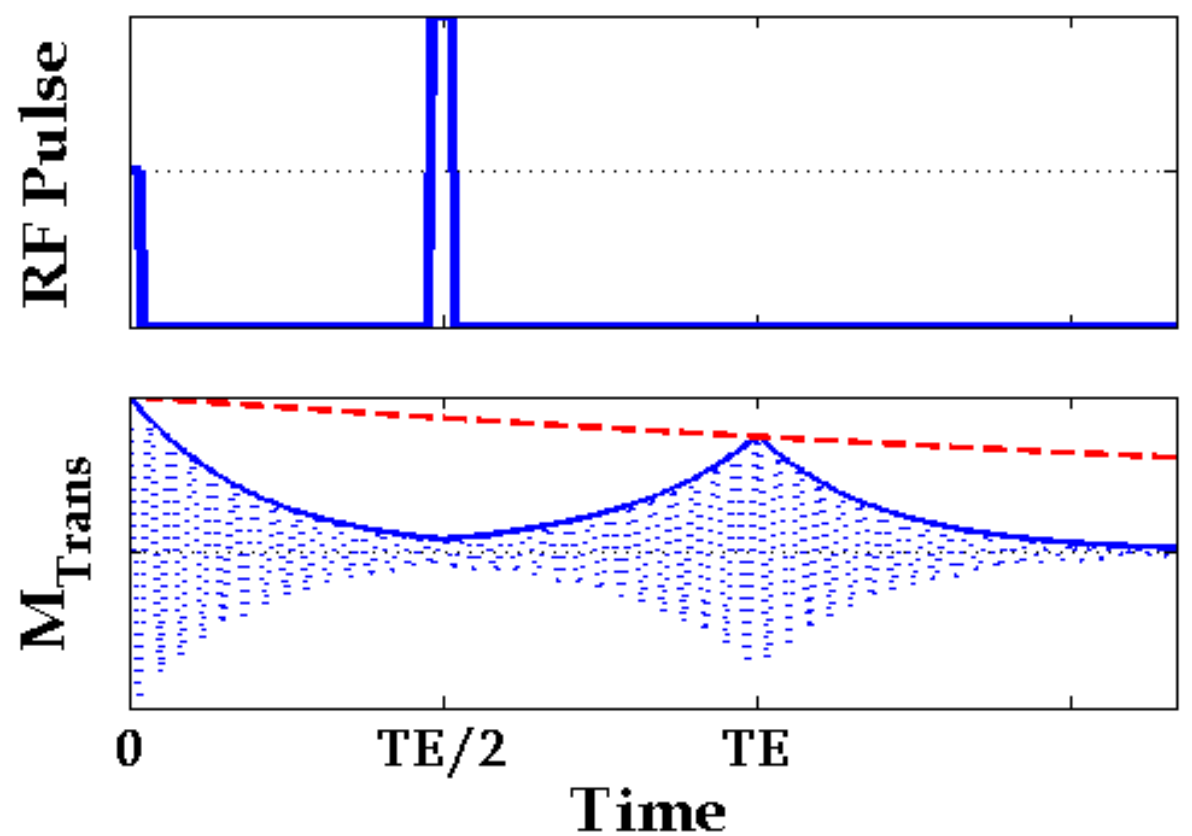

Figure 2.5: Transverse magnetization evolution to produce a spin echo. The signal is measured at the echo time $t=T E$, and the $180^{\circ}$ pulse is applied at $t=T E / 2$. The red dashed line shows the $T_{2}$ decay rate, the dashed blue line shows the laboratory signal, and the solid blue line is the "rotating frame" or demodulated signal. 


\subsection{Imaging}

In NMR, the precession frequency of nuclear spins depends on the applied magnetic field. By adding a magnetic field gradient $\vec{G}(\vec{r}, t)$ to the main field, the spin's frequency can be used to identify its location along the gradient direction. The added magnetic field is in the same direction as the main field ( $\hat{z}$ in our case), but the size of the field varies linearly with position along the gradient axis. For a time-dependent gradient along the $\hat{x}$ direction, the total applied field is given by:

$$
\vec{B}(x, t)=\left(B_{0}+G_{x}(t) * x\right) \hat{z}
$$

where here $G_{x}(t)=\frac{\partial B_{z}(t)}{\partial x}$ is the magnetic field gradient in the $\hat{x}$ direction.

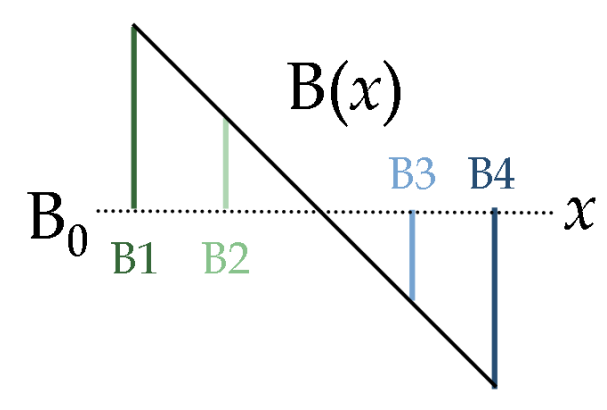

(a) Magnetic field as a function of position.

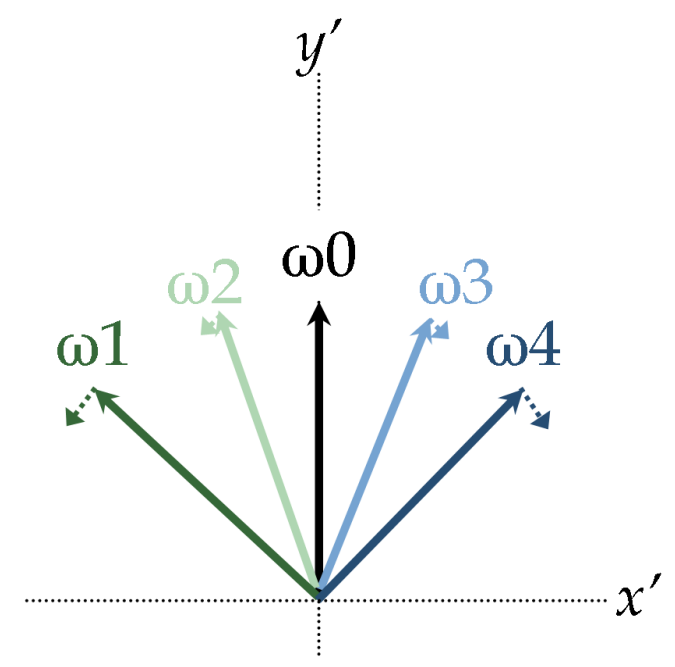

(b) Phase of spins.

Figure 2.6: Phase dispersion and precession frequency due to a magnetic field gradient. The gradient $G(x)$ alters the holding field value experienced by the spins based on their $\hat{x}$ location to be either greater than or less than $B_{0}$, which leads to a precession frequency greater than or less than $\omega_{0}$.

Across the sample along the gradient axis, the magnetic field will vary from less than $B_{0}$ to greater than $B_{0}$, as shown in Figure 2.6a. The spins at the different 
locations precess at different frequencies (Figure 2.6b). When the signal is measured (and the $\omega_{0}$ component factored out), it consists of components at each of the different frequencies:

$$
S(t)=\int M(x) e^{-i \gamma G x t} \mathrm{~d} x,
$$

where $M(x)$ is the position-dependent magnetization. The amplitude of a particular component frequency is proportional to the density of spins at the location which corresponds to that frequency, and this amplitude can be recovered by taking the Fourier transform of the acquired signal. It is convenient to replace time as the Fourier variable linked to position with the quantity:

$$
k \equiv \frac{\gamma}{2 \pi} \int_{0}^{t} G\left(t^{\prime}\right) \mathrm{d} t^{\prime}
$$

The variable $k$ corresponds to a spatial frequency. For the case of a constant gradient in one dimension, $k=\frac{\gamma G t}{2 \pi}$, which we can substitute into Equation 2.45:

$$
S(k)=\int M(x) e^{-i 2 \pi k x} \mathrm{~d} x
$$

By sampling many values of $k$, we can obtain the magnetization density through an inverse Fourier transform of the signal:

$$
M(x)=\int S(k) e^{i 2 \pi k x} \mathrm{~d} k
$$

To create a nuclear magnetic resonance image, magnetic field gradients are applied in multiple directions,

$$
\vec{k}(t)=\frac{\gamma}{2 \pi} \int_{0}^{t} \vec{G}\left(t^{\prime}\right) d t^{\prime}
$$


where

$$
\vec{G}(t)=\frac{\partial B_{z}(t)}{\partial x} \hat{x}+\frac{\partial B_{z}(t)}{\partial y} \hat{y}+\frac{\partial B_{z}(t)}{\partial z} \hat{z},
$$

and the signals obtained at the different gradient strengths are combined.

The process of sampling at different values of spatial frequency is referred to as covering $k$-space. The correspondence between $k$-space acquisition and image formation can be understood as follows: when a gradient is applied, there will be a pattern of signal coherence across the sample that depends on the phase accumulation at each location, which is related to the strength and duration of the magnetic field gradients. Figure $2.7(\mathrm{a}-\mathrm{c})$ illustrate several different signal coherence patterns, and the corresponding $k$ values are marked in (d) by the large circles. The black arrows represent a typical $k$-space acquisition scheme, where the k-space data for each line is acquired sequentially from bottom to top to cover a range of spatial frequencies.

Figure 2.8 is a pulse sequence diagram, which illustrates the timing of an MRI acquisition. The time between successive RF pulses is known as the repetition time $T R$. The area under the curve of the gradient is the gradient moment, which determines the region of $k$-space that is sampled during each acquisition. The most common method of covering a two-dimensional spatial frequency range is Cartesian sampling shown in Figure $2.7 \mathrm{~d}$, in which the $\hat{y}$ gradient is stepped from the largest negative value to the largest positive value in constant intervals for each $\mathrm{TR}$, and is referred to as the phase-encode gradient $\left(G_{P E}\right)$ in Figure 2.8. Each $\hat{y}$ step in the phase-encode gradient yields data on a line in k-space as the $\hat{x}$, or read-out gradient $G_{\text {Read }}$ is swept negative to positive. The data acquisition window (DAQ) is open during the read-out gradient sweep, and the echo time TE refers to the time point at which the $\hat{x}$ gradient moment is zero. To provide selectivity in the $\hat{z}$ direction, either a slice selective gradient $G_{\text {slice }}$ can be applied during the RF 

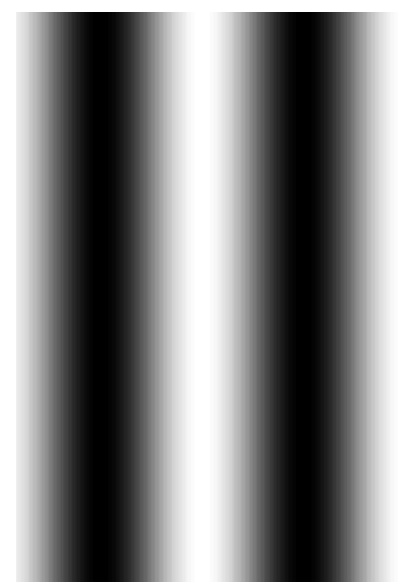

(a) Small Gradient in $\hat{x}$ (green point).

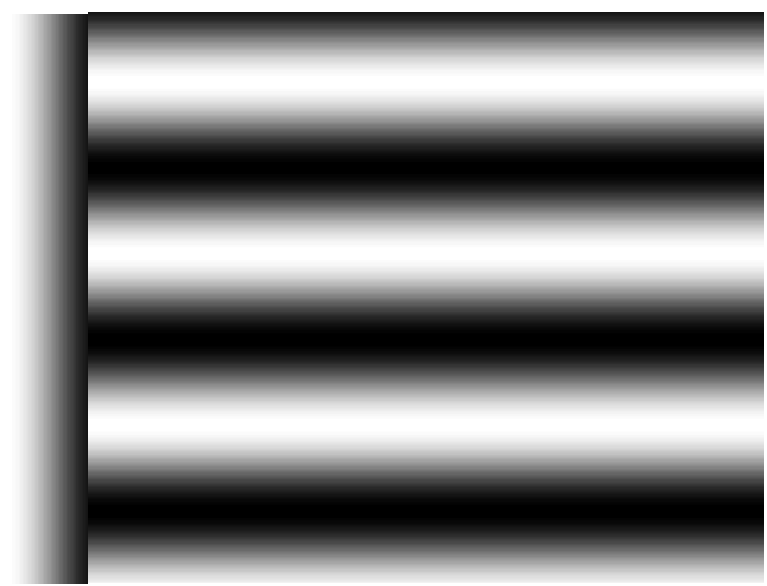

(b) Small Gradient in $\hat{y}$ (red point).

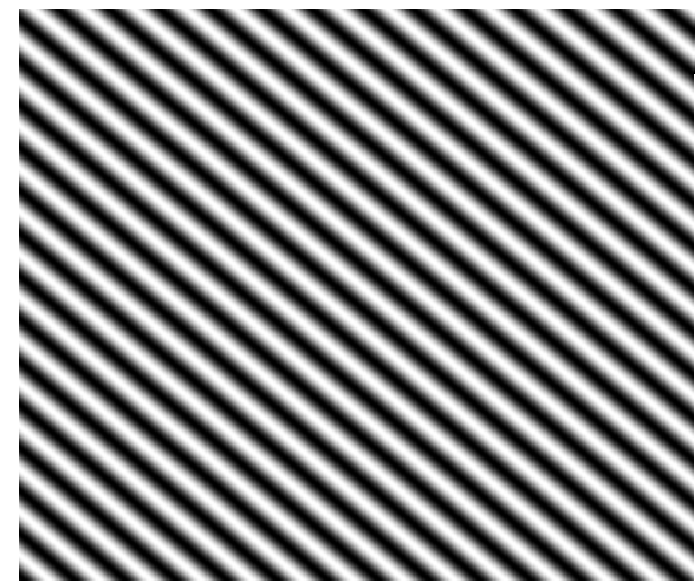

(c) Large Gradient in both $\hat{x}$ and $\hat{y}$ (blue point).

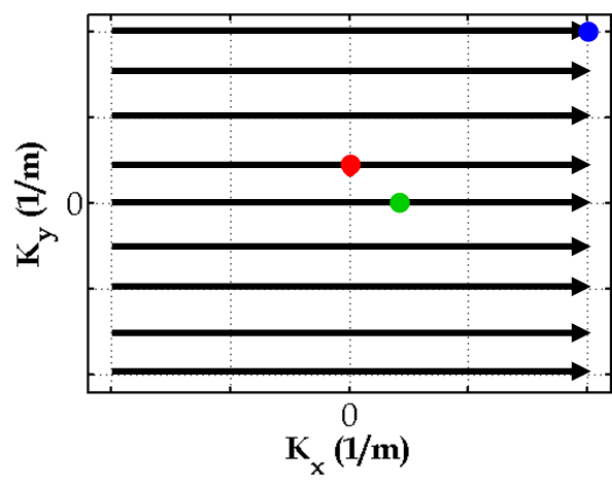

(d) Points on K-Space plane

Figure 2.7: Sample phase coherence maps and the corresponding k-space points. (a) A weak gradient in $\hat{x}$ (green point) produces a broad vertical coherence pattern, (b) a weak gradient in $\hat{y}$ (red point) produces a broad horizontal coherence pattern, (c) a strong combined gradient (blue point) produces a fine diagonal coherence pattern. 
pulse which causes only a segment of the particles to have their magnetizations tipped into the transverse plane (as shown in Figure 2.8), or $\hat{z}$ gradients can also be stepped through similar to $\hat{y}$ gradients (not shown, referred to as partition-encode gradients).
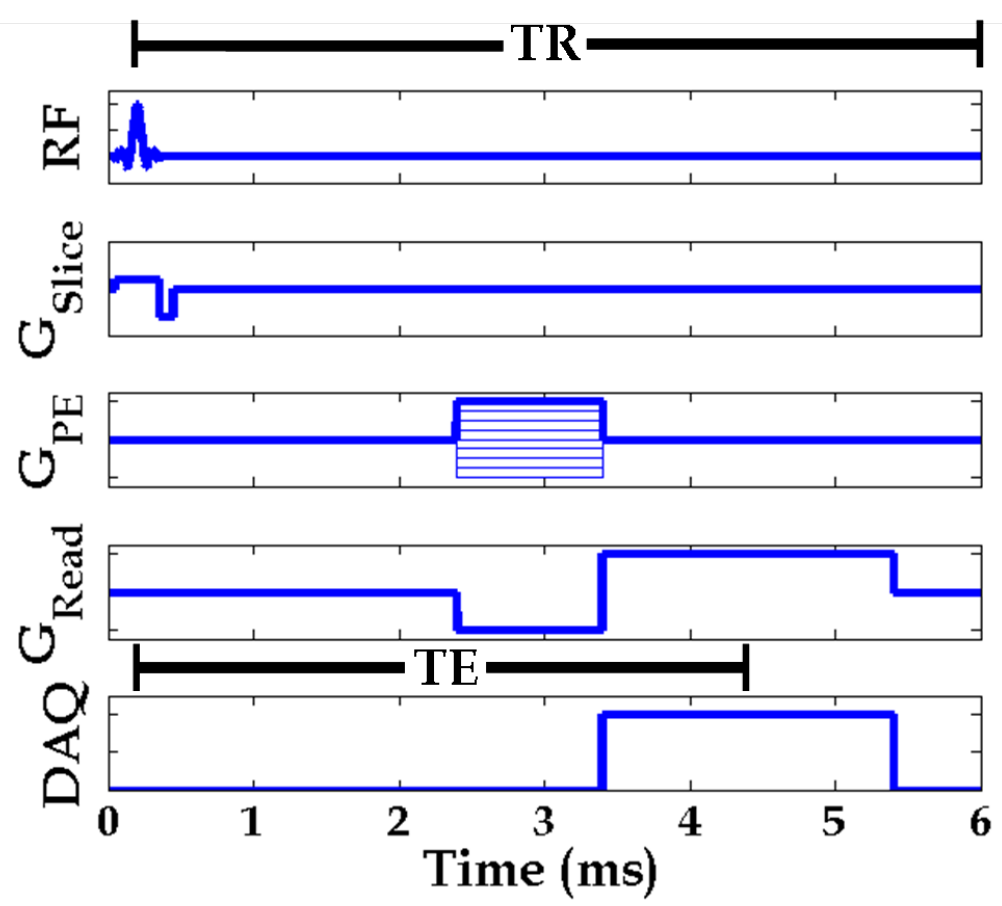

Figure 2.8: A magnetic resonance image pulse sequence diagram. The RF application repeated every $t=T R$ tips the magnetization into the transverse plane. The gradient applied during the RF pulse, $G_{\text {Slice }}$, selects a portion of the sample to be measured along the gradient direction. Phase-encode (PE) and read-out (Read) gradients are applied to induce precession frequency differences across the sample. The data acquisition (DAQ) is centered at $t=T E$.

The signal size $S(\vec{k})$ measured for each value of $k$ applied during the acquisition is related to how closely the coherence pattern created by the gradients matches features found in the sample,

$$
S(\vec{k})=\int M_{T}(\vec{r}) e^{-i 2 \pi \vec{k} \cdot \vec{r}} d \vec{r},
$$

where $M_{T}(\vec{r})$ is the transverse magnetization. 
Figure 2.9a shows k-space data from an MRI acquisition. This image is formed by displaying the natural $\log$ of the signal size versus the $k$-value to bring out the low values on the edges of $k$-space. Central $k$-space points have low gradient strengths, and points away from the center have large gradient strengths. By taking the Fourier transform of the $k$-space data, the relative contribution from each spatial frequency can be determined:

$$
M_{T}(\vec{r})=\int S(\vec{k}) e^{i 2 \pi \vec{k} \cdot \vec{r}} d \vec{k}
$$

The final image is a coherent superposition of all of the signal interference patterns produced by the gradients.

The equations above treat $k$ as a continuous variable, but in practice it is stepped through discretely. The pattern made by stepping through k-space determines several factors about the reconstructed image. Large gradients produce rapid changes in phase, which will result in high resolution in the final image. Very fine steps in k-space or gradient strength allow a large field of view to be included in the image. Figure $2.9 \mathrm{~b}$ is the Fourier transform of the data in Figure 2.9a, an MRI of the torso of a volunteer imaged for this work. The image shows bright signal in the heart and major blood vessels. Also present in the field of view are various muscles, organs, bone, and fat, each with varying degrees of brightness. The lung tissue region is dark, as the density of hydrogen nuclei is too low in this region to generate measurable signal.

MR imaging of the body provides information about the density of hydrogen nuclei in a particular region. There are a variety of techniques that can be applied to gain more information from the image than spin density. Diffusion MR is capable of distinguishing particles based on how far they have moved during the imaging process. This technique will be the topic of Section 2.5. 


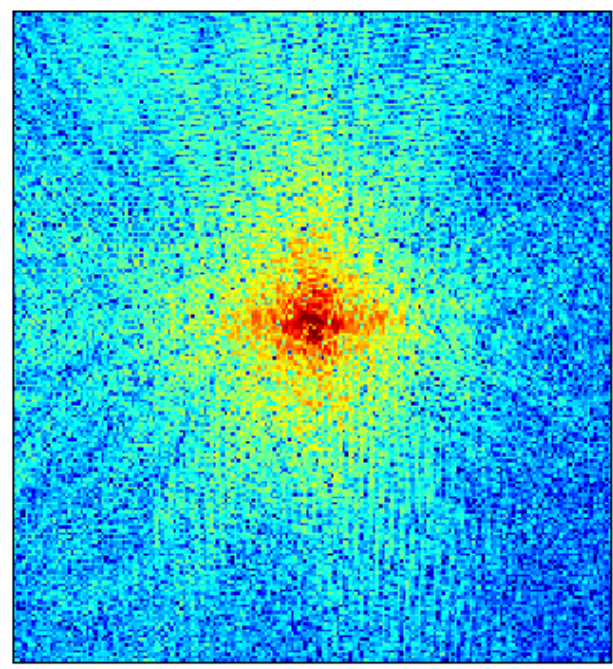

(a) K-Space data.

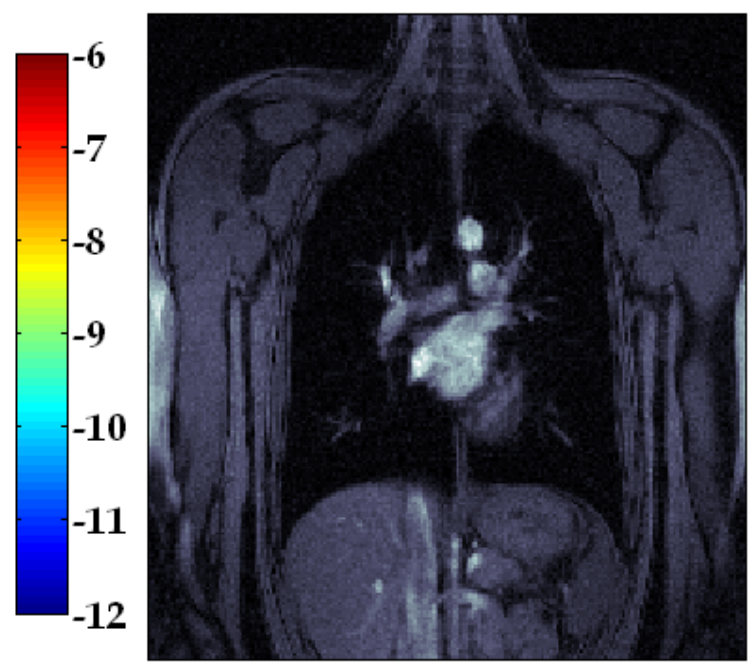

(b) MRI.

Figure 2.9: (a) K-space data (displayed on a logarithmic scale to enhance low values): The signal at each point determines how much the corresponding spatial frequency contributes to the final image. (b) The Magnetic Resonance Image shows the density of ${ }^{1} \mathrm{H}$ spins in each location, and it is the Fourier transform of the kspace data shown in (a).

\subsection{Hyperpolarized Gas MRI}

Lung tissue has a low density of hydrogen nuclei compared to other tissue in the body, making it difficult to image with conventional ${ }^{1} \mathrm{H}$ MRI. Hyperpolarized gas MRI is a technique in which the airspaces in the lungs can be imaged after a subject inhales a noble gas which has been polarized above the thermal equilibrium value [2].

Both helium-3 and xenon-129 are commonly used as imaging gases [25], but the work in this thesis focused on helium-3 as the target for MR. The process of producing highly polarized helium-3 is the subject of Chapter 3 . The signal increase from thermal equilibrium polarization to hyperpolarization allows for a compensation of the density reduction when switching from hydrogen nuclei to gases. Table 2.3 lists the densities and polarizations for hydrogen nuclei in body tissue and 


\begin{tabular}{|c|c|c|}
\hline Nucleus & Density & $\mathrm{P}$ \\
\hline${ }^{1} \mathrm{H}$ & $10^{5} \mathrm{~mole} / \mathrm{cm}^{3}$ & $P_{\text {Thermal }}=10^{-6}$ \\
\hline${ }^{3} \mathrm{He}$ & $50 \mathrm{~mole} / \mathrm{cm}^{3}$ & $P_{H P}=0.1-1$ \\
\hline
\end{tabular}

Table 2.3: Density and polarization for hydrogen nuclei in body tissue and hyperpolarized helium-3 gas.

helium-3 gas in the lung. The density reduction between liquid to gas is a factor of $10^{3}-10^{4}$, whereas the polarization increase from thermal equilibrium magnetization to hyperpolarized magnetization contributes a factor of $10^{6}$. Magnetization is proportional to the product of density and polarization (Equation 2.33), meaning hyperpolarized gas MR has the potential to generate a larger magnetization (and thus a larger signal) than conventional MR with thermally polarized hydrogen nuclei.

There are several differences between hyperpolarized gas MR and conventional ${ }^{1} \mathrm{H} \mathrm{MR}$, the first being the time evolution of the longitudinal magnetization. In both cases, the magnetization approaches the thermal equilibrium value with the time constant of $T_{1}$. However, in the case of hydrogen nuclei the magnetization increases towards thermal equilibrium while the hyperpolarized helium-3 magnetization decreases from the hyperpolarized value towards thermal equilibrium as illustrated in Figure 2.10.

The signal in conventional MR can be considered renewable as it regrows toward equilibrium after each RF pulse. By waiting a few seconds in between excitations, the polarization can recover to the maximum level, depending on $T_{1}$. In contrast, the hyperpolarized signal is non-renewable and decays in the lung with $T_{1} \approx 20 \mathrm{~s}$. The first consequence of this signal behavior is that data acquisition must be completed before the signal has decayed. Since hyperpolarized gas MR in vivo is typically performed at breath-hold, acquisition times are further limited 


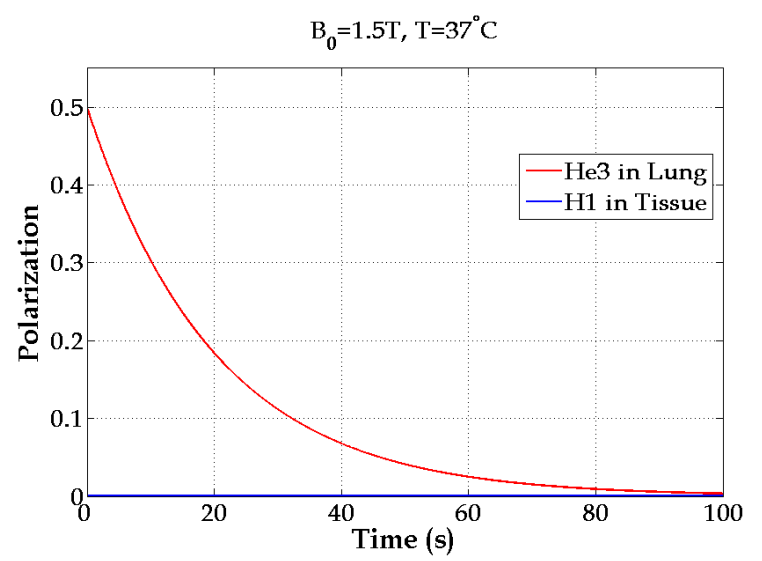

(a)

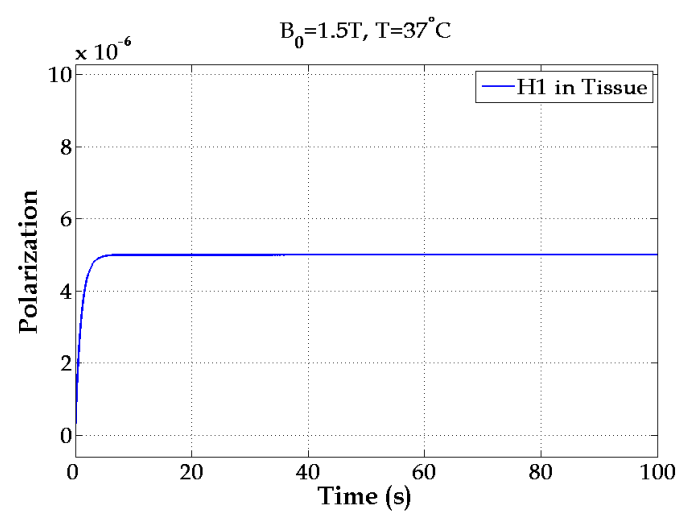

(b) (a) zoomed in to show ${ }^{1} \mathrm{H}$.

Figure 2.10: Time evolution of longitudinal magnetization in thermal equilibrium and hyperpolarized cases. (a) In the lung, the helium-3 signal decays from hyperpolarized to thermal equilibrium polarization $P_{\text {Thermal }}(\mathrm{He} 3)=7 \times 10^{-6}$ with the time constant $T_{1}=20 \mathrm{~s}$. (a) In body tissue, the hydrogen signal grows towards thermal equilibrium $P_{\text {Thermal }}(H 1)=5 \times 10^{-6}$ with a time constant $T_{1}=1 \mathrm{~s}$.

by this requirement to $\leq 20 \mathrm{~s}$ in healthy subjects, whereas $\leq 10 \mathrm{~s}$ is preferable for subjects with impaired lung function.

An additional consideration when working with hyperpolarized magnetization is the size and duration of the RF excitation pulses used to tip longitudinal magnetization into the transverse plane. As discussed in Section 2.2, the flip angle $\theta$ is determined by the RF pulse amplitude and duration, and the signal measured is proportional to $\sin \theta$, with the remaining longitudinal magnetization proportional to $\cos \theta$. In conventional MR it is common to use $\theta \sim 90^{\circ}$ and a long repetition time to generate the maximum transverse signal.

For hyperpolarized gas MR, every RF pulse diminishes the available longitudinal magnetization, so low flip angles are typically used so as not to exhaust the available magnetization too quickly. An efficient flip angle choice would reduce the longitudinal magnetization at such a rate that the signal is nearly gone by the last excitation. The optimal flip angle can be calculated based on the number of excitations in the pulse sequence [26]. 
The gyromagnetic ratio is lower for helium-3 than hydrogen nuclei, meaning that the spins precess at a lower frequency in the same holding field generated (see Table 2.1). For conventional MR, the RF excitations and data acquisition are executed by magnetic coils and amplifiers that commonly have a narrow operating frequency range around the ${ }^{1} \mathrm{H}$ resonance frequency to reduce noise. In order to perform hyperpolarized gas MR on a clinical scanner dedicated amplifiers and coils for helium-3 must be built or purchased. Figure 2.11 shows two examples of helium-3 coils used in this work, a homebuilt birdcage-style coil [27] used for phantom studies, and a commercial coil used with human subjects (Rapid Biomedical, Rimpar Germany).

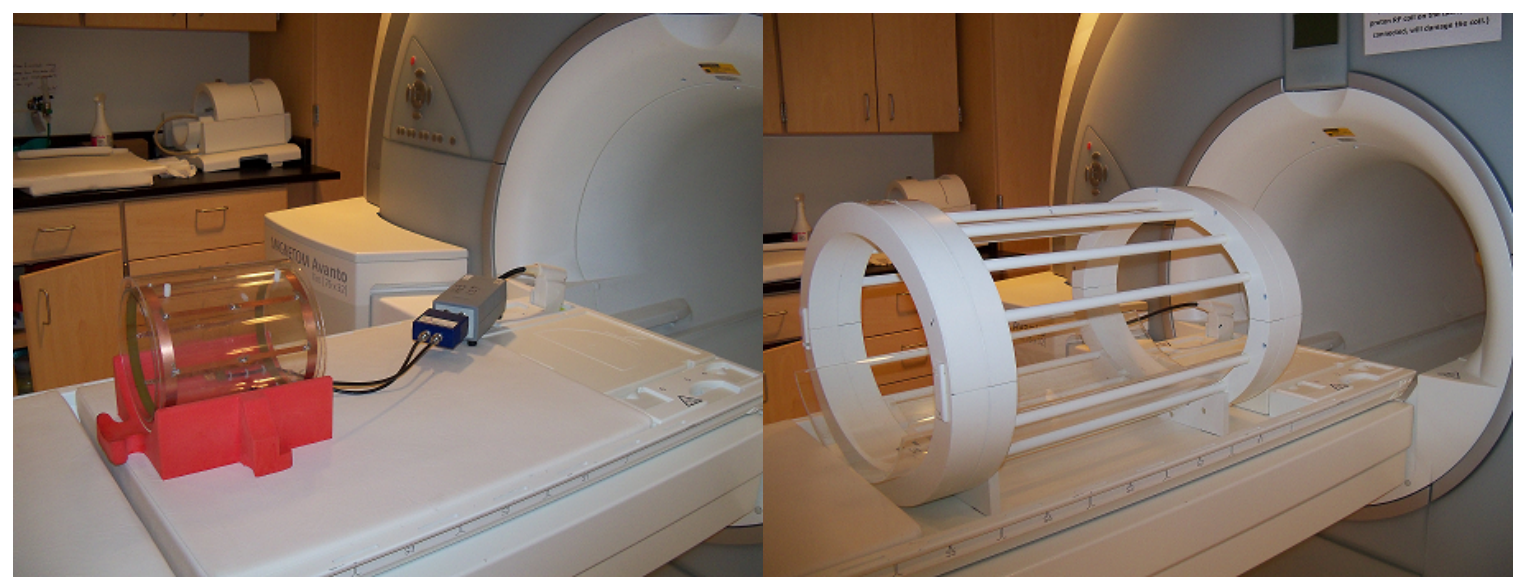

(a) Helium-3 Coil for phantoms.

(b) Helium-3 Coil for subjects.

Figure 2.11: Specialized coils used for MRI with hyperpolarized helium-3. (a) Homebuilt birdcage-style coil used in phantom studies. (b) Commercial coil used with human subjects.

An MR image acquired with hyperpolarized gas is shown in Figure $2.12 \mathrm{~b}$, along with a conventional ${ }^{1} \mathrm{H}$ image taken in the same subject. Both images were acquired at breath hold, with the subject inhaling a dose of hyperpolarized xenon129 prior to the gas image. The conventional image has dark regions where the lungs are located, due to the low density of tissue this area. The hyperpolarized 
gas image shows the ventilated airspaces of the lung, so this type of image is often referred to as a ventilation image.

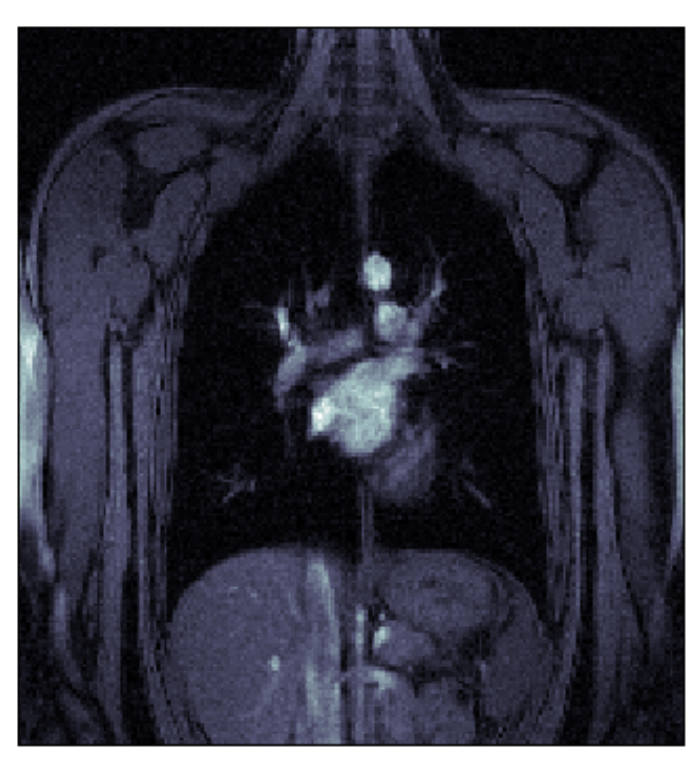

(a) ${ }^{1} \mathrm{H}$ MRI

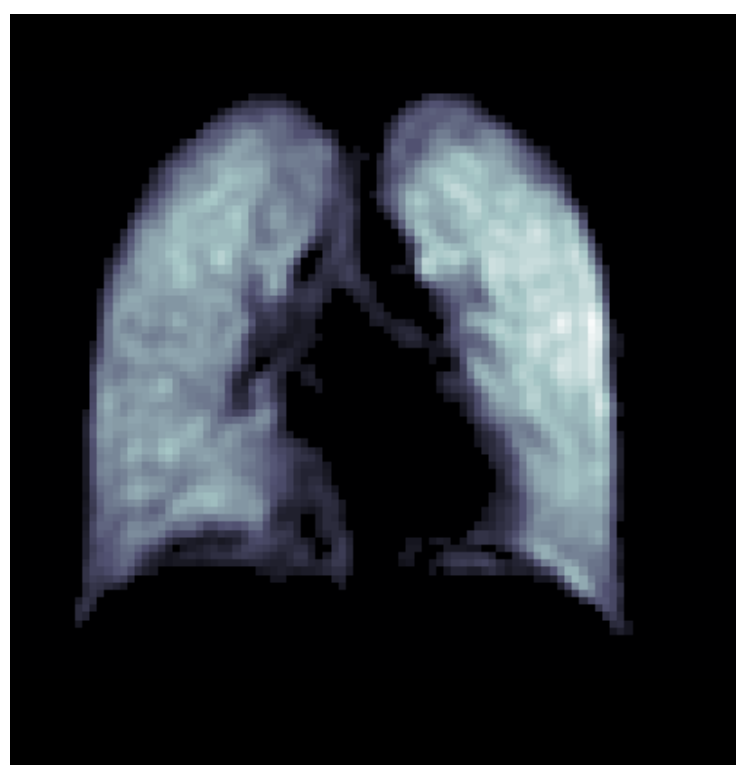

(b) $\mathrm{HP}^{129}$ Xe MRI

Figure 2.12: ${ }^{1} \mathrm{H}(\mathrm{a})$ and hyperpolarized ${ }^{129} \mathrm{Xe}(\mathrm{b}) \mathrm{MR}$ images of a healthy volunteer. The lung tissue contributes no signal to the conventional MRI, but the lung airspaces can be imaged by inhaling hyperpolarized gas prior to imaging.

The resolution for 2-D human ventilation images is typically a few millimeters in the plane of the image, with slices $10-20 \mathrm{~mm}$ thick. The ventilation image provides regional information regarding both lung structure and function. This type of image is very useful for showing regional ventilation patterns in diseases such as asthma [28] and cystic fibrosis [5], where poorly ventilated areas can be identified by the lack of helium-3 signal. For a disease like emphysema, ventilation imaging alone provides a resolution that is too coarse to directly observe tissue destruction. The next section describes diffusion-weighted imaging, which allows the fine structure of the lung below the resolution limit to be probed, based on its influence on gas diffusion. 


\subsection{Diffusion-weighted Imaging}

Throughout the course of any MR acquisition, the particles being detected (liquid water in the case of ${ }^{1} \mathrm{H} \mathrm{MR}$, or helium-3 atoms in the case of hyperpolarized gas MR) are constantly moving. As the particles undergo this random diffusion, they experience slightly different magnetic fields at different times due to magnetic field inhomogeneities. The different field values alter the spins' precession frequencies, which results in an accumulation of relative phase between different particles that reduces net signal coherence. The farther particles move in a given period, the more field variation they can experience, resulting in more phase dispersion. Since the motion is random, the phase dispersion cannot be reversed by the application of spin echo pulses. This effect is present to some degree in every MR acquisition, and effectively shortens $T_{2}$.

Applied magnetic field gradients also produce a location-based frequency dependence resulting in phase dispersion. This phenomenon is exploited in diffusionweighted MRI. Figure 2.13 illustrates the relationship between gradient application and phase dispersion, where in $\left(\mathrm{a}, \mathrm{c}\right.$, and e) the label $B_{i}$ indicates the field at the $\hat{x}$ location of particle $i$. When a linear magnetic field gradient $+G$ is applied (Figure 2.13a), the particles accumulate relative phase according to their location (Figure 2.13b). In the absence of diffusion, the phase dispersion can be reversed by the application of the opposite gradient $-G$, as shown in Figure 2.13c-e. If instead the spins are allowed to move during the time between the application of $G$ and $-G$, some will not experience the exact opposite magnetic field value (Figure 2.13fg). During the application of $-G$, the spins alter their precession frequencies, but the new frequencies do not bring spins which have moved between $+G$ and $-G$ back into full coherence, as shown in Figure 2.13h.

Diffusion-sensitization is created in hyperpolarized gas MR through the ap- 


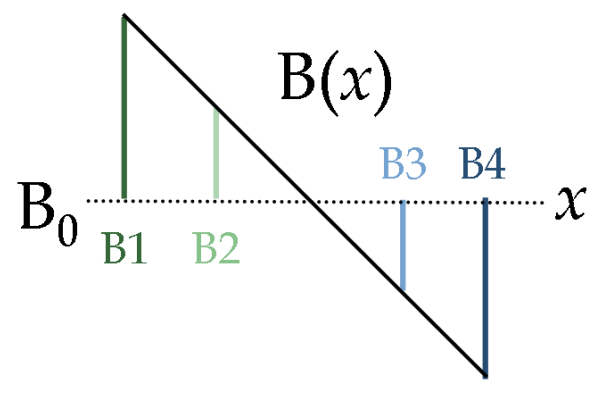

(a) Magnetic field as a function of position when gradient pulse $+G$ is applied.

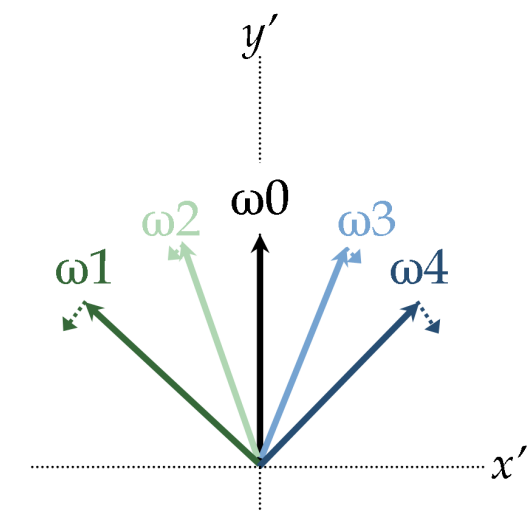

(b) Phases and precession frequencies of spins after gradient pulse $+G$ has been applied.

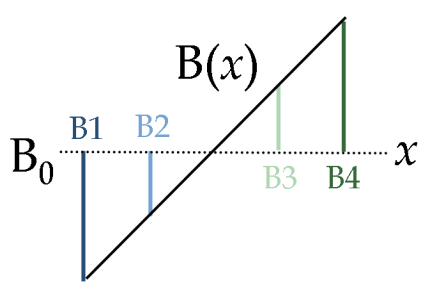

(c) Magnetic field as a function of position when gradient pulse $-G$ is applied.

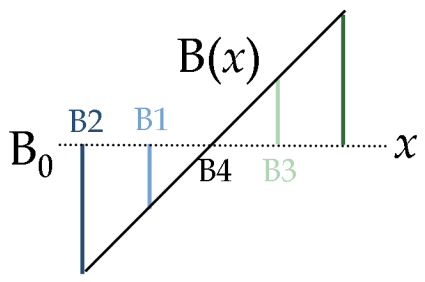

(f) Magnetic field as a function of position when gradient pulse $-G$ is applied in the presence of diffusion.

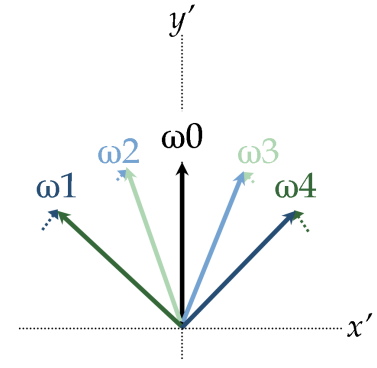

(d) Phases and precession frequencies during application of gradient pulse $-G$.

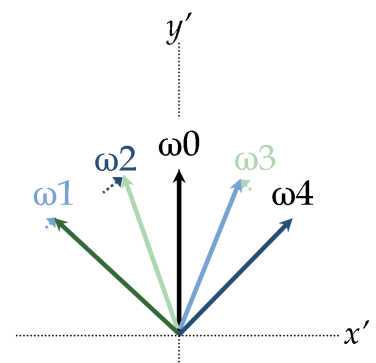

(g) Possible phases and precession frequencies during application gradient pulse $-G$ in the presence of diffusion.

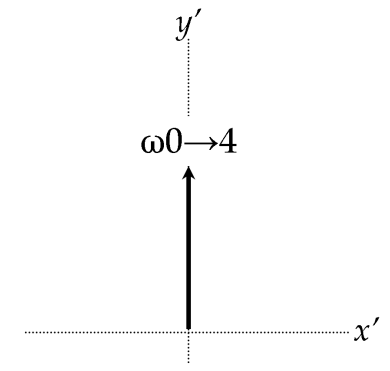

(e) Phases and precession frequencies after gradient pulse $-G$ has been applied.

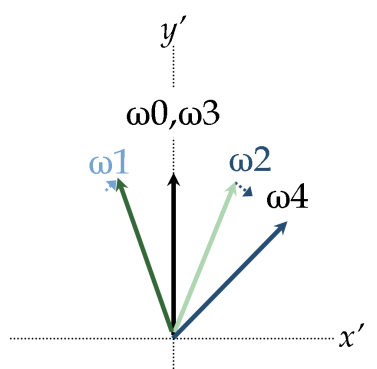

(h) Possible phases and precession frequencies after gradient pulse $-G$ has been applied in the presence of diffusion.

Figure 2.13: Magnetic field gradients and phase accumulation. (a) A gradient pulse $+G$ alters the magnetic field based on position, such that after it is applied spins have different precession frequencies (b). Subsequently, the opposite gradient pulse $-G(\mathrm{c})$ reverse the precession frequencies of the spins (d) in the absence of diffusion, resulting in total coherence (e). If some spins diffuse before the application of the reversed gradient pulse $-G(\mathrm{f})$, the spins do not all reverse the accumulated phase completely $(\mathrm{g})$, resulting in a diminished total signal $(\mathrm{h})$. 
plication of a bipolar diffusion gradient, which consists of a gradient with positive (or negative) moment followed by a gradient with the equal and opposite moment. During a diffusion-weighted MR acquisition shown in Figure 2.14, a bipolar diffusion gradient $\left(G_{D i f f}\right)$ is applied before the signal is measured in one of the two component images [29]. The time that separates the two halves of the diffusion gradient is the diffusion time, referred to here as $\Delta$. The net phase accumulation due to this gradient pair will be zero for a particle that has not moved during $\Delta$, and non-zero for a particle that has moved.

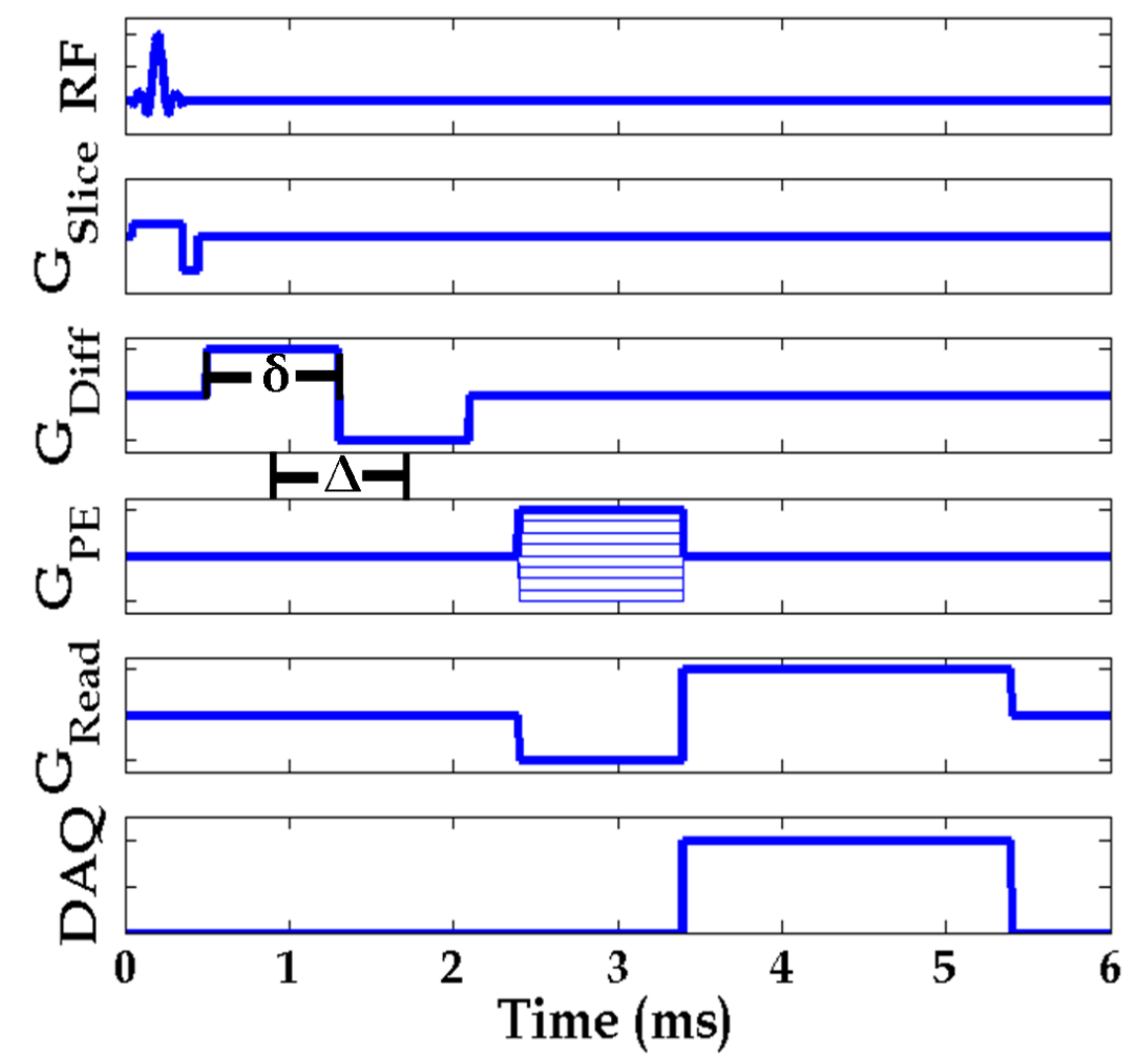

Figure 2.14: A standard Diffusion-weighted MRI pulse sequence diagram for use with hyperpolarized gas. In addition to the RF pulse and imaging gradients used in conventional MRI, a bipolar diffusion gradient $G_{D i f f}$ is applied before the data acquisition. The image acquired with this pulse sequence is compared to an image acquired without any diffusion gradients. The diffusion gradient can be applied on any physical axes.

The measured signal is reduced due to the phase dispersion by an amount that 
depends on the diffusivity $D$ as well as characteristics of the gradients being applied. After the application of a diffusion-sensitizing gradient $G(t)$, the transverse magnetization is modified from Equation 2.42 as follows:

$$
M_{T}(t)=M_{T}(0) e^{-\left(i \omega_{0}+\frac{1}{T_{2}^{*}}\right) t} e^{-b D},
$$

where

$$
b \equiv \gamma^{2} \int_{0}^{t} \mathrm{~d} t^{\prime \prime}\left(\int_{0}^{t^{\prime \prime}} \vec{G}\left(t^{\prime}\right) \mathrm{d} t^{\prime}\right)^{2} .
$$

The variable $b$ parameterizes the amount of diffusion attenuation induced by a specific gradient. The equations defining $b$ in terms of the parameters describing several possible single-axis bipolar gradient configurations shown in Figure 2.15 are given in Table 2.4 [23].

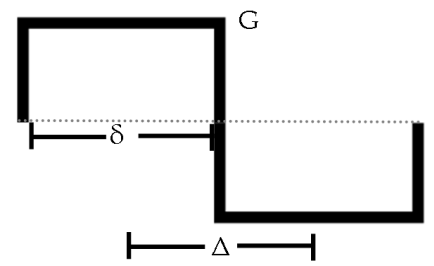

(a) Rectangular Gradient Pulse

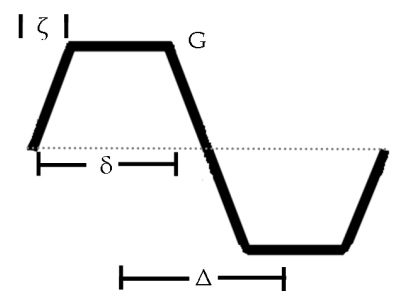

(b) Trapezoidal Gradient Pulse

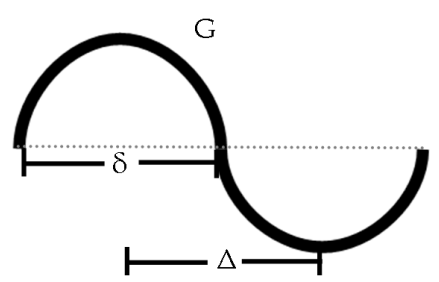

(c) Sinusoidal Gradient Pulse

Figure 2.15: Gradient pulse shapes (a) rectangular, (b)trapezoidal, and (c) sinusoidal. $\Delta$ is the diffusion time, the time between lobe centers. $\delta$ is the lobe duration, $G$ is the magnitude, and $\zeta$ is the trapezoidal ramp up time.

In practice, the signal is measured in scans with $\left(S_{1}\right)$ and without $\left(S_{0}\right)$ applied diffusion gradients. The ratio of the two signals can be used extract the ADC of the particles in the space, either on a pixel by pixel basis in an MRI or in a global NMR measurement using Equation 2.53:

$$
A D C=-\frac{\log \frac{S_{1}}{S_{0}}}{b} .
$$




\begin{tabular}{|c|c|}
\hline Shape & Equation \\
\hline Rectangular & $b=\gamma^{2} G^{2} \delta^{2}(\Delta-\delta / 3)$ \\
\hline Trapezoidal & $b=\gamma^{2} G^{2}\left[\delta^{2}(\Delta-\delta / 3)+\zeta^{3} / 30-\delta \zeta^{2} / 6\right]$ \\
\hline Sinusoidal & $b=4 \pi^{-2} \gamma^{2} G^{2} \delta^{2}(\Delta-\delta / 4)$ \\
\hline
\end{tabular}

Table 2.4: B-Values for Common Gradient Shapes $\gamma$-gyromagnetic ratio, G-Gradient Amplitude, $\delta$-Gradient lobe duration, $\Delta$-Time between lobe centers, $\zeta$-Trapezoidal Gradient ramp time

Figure 2.16 shows an example of an ADC map acquired with hyperpolarized helium3 in a healthy subject. Two ventilation image acquisitions were interleaved line by line, with one containing a trapezoidal diffusion gradient with $\Delta=1.58 \mathrm{~ms}$, $\zeta=300 \mu \mathrm{s}$, and $b=1.6 \mathrm{~s} / \mathrm{cm}^{2}$ between the excitation and read-out gradient, as shown in Figure 2.14. The in-plane resolution of the images is $6.6 \times 6.6 \mathrm{~mm}$ and the slice thickness is $25 \mathrm{~mm}$.
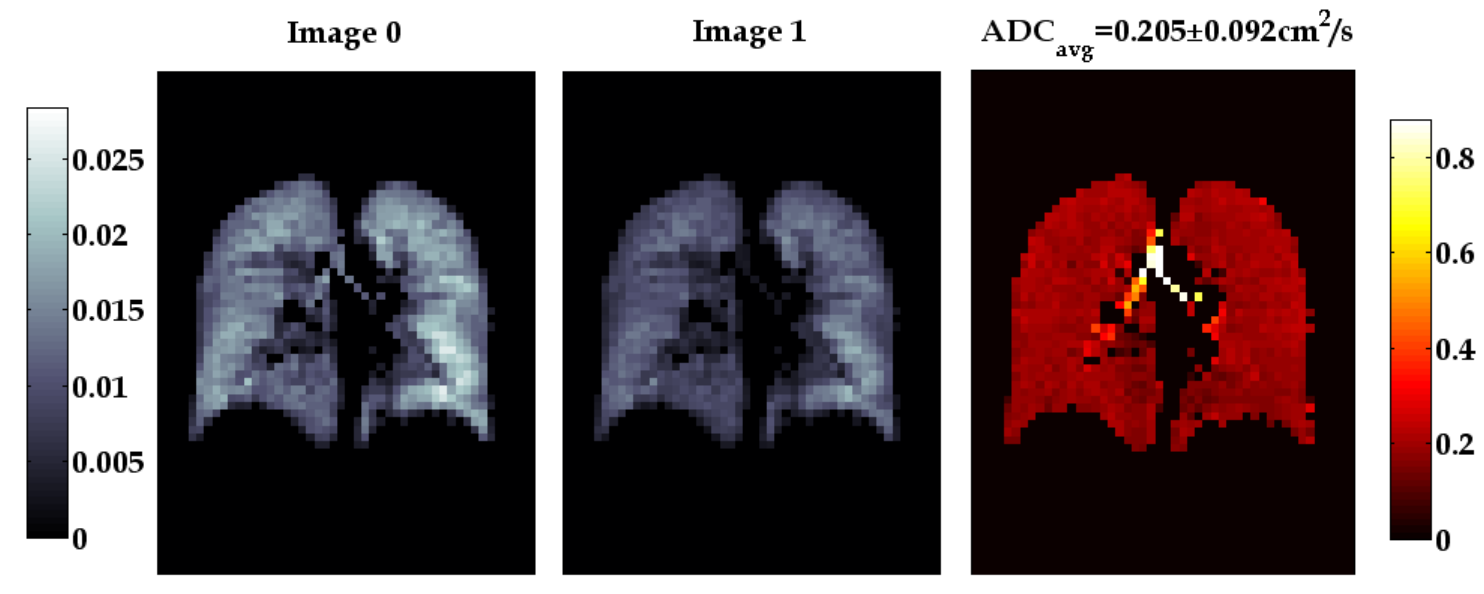

Figure 2.16: Diffusion-weighted MRI of the lung in a healthy volunteer. The images are acquired without diffusion gradients (Image 0 ) and with diffusion gradients (Image 1). The ADC map is calculated from the ratio of the two images using the b-value associated with the gradient applied. The data was acquired using a diffusion time of $\Delta=1.58 \mathrm{~ms}, b=1.6 \mathrm{~s} / \mathrm{cm}^{2}$. The measured values show low diffusion $\left(A D C \sim 0.2 \mathrm{~cm}^{2} / \mathrm{s}\right.$ ) in the alveoli where the gas is very restricted, and high diffusion $A D C \sim 0.8 \mathrm{~cm}^{2} / \mathrm{s}$ in the trachea where the gas is free to move during the scan. 
There is a general signal decline between Image 0 and Image 1 due to diffusion attenuation. The largest difference between the two images appears in the airways, which are barely visible in the second image. The ADC was calculated pixel by pixel from the two images using Equation 2.55. The measured values of the apparent diffusion coefficient reveal information about the lung structure, as the airway values of $A D C \sim 0.8 \mathrm{~cm}^{2} / \mathrm{s}$ indicate nearly free diffusion in this region and the values of $A D C \sim 0.2 \mathrm{~cm}^{2} / \mathrm{s}$ in the bulk of the lung suggest a very restrictive diffusing environment.

By measuring diffusion coefficients in healthy and diseased lungs, characteristics of the lung airspaces can be determined. In healthy lungs $\sim 95 \%$ of the total gas is in the alveoli, which have a very uniform structure of small airspaces and therefore are highly restrictive to gas diffusion, resulting in low diffusion coefficients. In emphysematous lungs, the walls between alveoli break down to create larger airspaces that are less restrictive to gas diffusion, resulting in larger values of $D$.

As mentioned in Chapter 1, in confined spaces the diffusivity depends on measurement time. For very short diffusion times particles away from the walls diffuse as if they were in free space, while particles near the walls are restricted in their movement. At long diffusion times, all of the particles will encounter a wall and be restricted. In a simple spherical model for the alveoli, the number of particles that are influenced by the wall is related to the proportion of particles that can strike a wall during the measurement time (Figure 2.17).

The diffusivity measurements are sensitive to different degrees of restriction, but in general that sensitivity cannot be translated into specific information about the environment. However Mitra, et al $[30,31]$ have shown that the diffusion coef- 


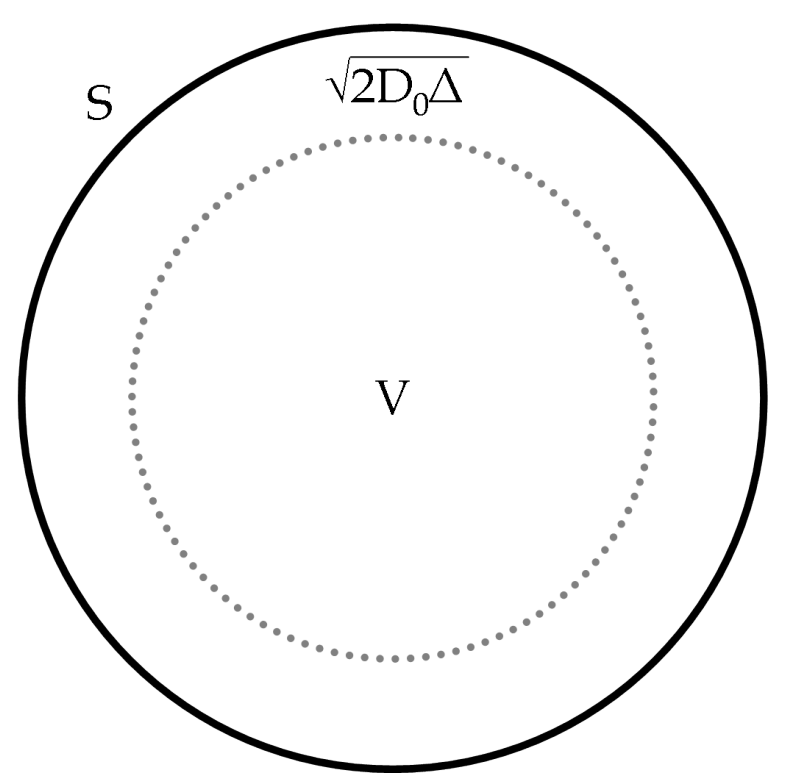

Figure 2.17: Simple model of diffusion in a sphere showing the striking distance to the surface. Spins in the center portion of the volume will not encounter a wall with a diffusion time of $\Delta$. Spins between the dotted line and the wall will be restricted, resulting in a lower measured ADC on the edges of the volume. As $\Delta$ increases, the restricted area grows and the average ADC decreases.

ficient behaves as follows:

$$
D(t)=D_{0}\left[1-\alpha \frac{S}{V} \sqrt{D_{0} \Delta}\right], \quad \alpha=\frac{4}{9 \sqrt{\pi}}
$$

for measurement times that fit the short-time scale criterion:

$$
\Delta \leq \frac{1}{\left(\frac{S}{V}\right)^{2} D_{0}}
$$

where $\Delta$ is the diffusion time, $S / V$ is the surface-to-volume ratio of the space, and $D_{0}$ is the free diffusion coefficient of the gas. In this region of diffusion times, the diffusivity can be directly related to structural parameters of the diffusing environment.

As the particles are allowed to diffuse for more time, eventually all of the particles will be within the striking distance of a wall and the time dependence of the 


\begin{tabular}{|c|c|c|c|}
\hline$\alpha_{\text {narrow }}$ & $\alpha_{\text {square }}$ & $\alpha_{\text {triangle }}$ & $\alpha_{\text {sine }}$ \\
\hline 0.253 & 0.317 & 0.297 & 0.298 \\
\hline
\end{tabular}

Table 2.5: $\alpha$ values for wide gradient pulses. $\alpha_{\text {narrow }}=\frac{4}{9 \sqrt{\pi}}$.

diffusivity approaches what is known as the tortuosity limit [32]:

$$
D(t \rightarrow \infty)=\frac{D_{0}}{\alpha}
$$

where $\alpha$ is the tortuosity of the space.

Previous work using both of these theoretical diffusivity curves to calculate structural parameters from gas diffusion measurements was pioneered by Mair et al [33-35]. Using glass bead phantoms and porous rock samples, Mair measured the time dependence of the xenon-129 diffusion coefficient, observing both the short-time scale $S$ / $V$ behavior and the long-time scale $\sqrt{\phi}$ behavior, with some important systematic effects. Equation 2.56 applies to porous media regardless of geometry, provided that the total system diameter $L$ is large compared to the bead diameter $d, L>>d^{2}$, and the gradient pulses fulfill the narrow-pulse criterion, $\delta<<\Delta$ [30]. When these conditions were violated, the measured ADC deviated from the expected values.

Carl et al were able to characterize effects due to violation of the narrow pulse criterion through simulations and experiments measuring the helium-3 diffusion coefficient in glass bead phantoms [36]. The use of wide pulses relative to the diffusion time can be compensated for by using a value of $\alpha$ appropriate for the pulse shape in equation 2.56. The $\alpha$ values for various gradient pulse shapes are listed in Table 2.5.

The goal of this thesis is to develop a method for making hyperpolarized gas diffusion measurements at diffusion times of $\Delta<1 \mathrm{~ms}$, where the diffusivity 
should be sensitive to a length scale closer to the alveolar size in humans. The short diffusion times impart a much lower diffusion attenuation than the conventional $\Delta$ values, due to the gradient slew-rate limitations on the scanner. To increase the net attenuation, a technique has been implemented by Carl et al in which short-duration gradients are repeated several times in the $T R$ period, with each still maintaining the short time-scale dependence [37].

This work explores an alternate method of measuring the small diffusion attenuation created by short diffusion times. Using a sequence technique known as steady-state free precession the sensitive measurement is made on a large, nearly constant signal. The full description of the method will be detailed in Chapter 4 . 


\section{Chapter 3}

\section{Hybrid Rubidium/Potassium Helium Polarizer for Medical Imaging}

In order to perform hyperpolarized-gas magnetic resonance imaging, it is necessary to produce a polarized noble gas. Polarization of noble gases is typically achieved by one of two processes- Spin-Exchange Optical Pumping (SEOP) [38] or Metastable Exchange Optical Pumping (MEOP) [39]. The first hyperpolarizedgas MR images were taken with ${ }^{129} \mathrm{Xe}$ at $P_{\mathrm{Xe}} \sim 25 \%$ polarization in 1994 [2]. Over the last two decades, helium-3 has become the more common gas for human lung imaging and until the work described in this chapter, the polarization performance was $P_{\mathrm{He}} \sim 40 \%$ in SEOP-based systems [3] and $P_{\mathrm{He}} \sim 50 \%$ in MEOP-based systems [40]. In the following chapter, we describe a new SEOP-based helium-3 polarizer for use in medical imaging experiments which produces $3 \mathrm{~L}$ of helium-3 at $P_{\mathrm{He}}>60 \%$, the Hybrid Polarizer.

In building a new SEOP ${ }^{3} \mathrm{He}$ polarizer, we looked to recent atomic and nuclear physics experiments for methods to increase performance. The most important advances incorporated into the hybrid polarizer are line-narrowed lasers, a hybrid alkali-metal mixture, and the benefits of extensive optimization. It is the combi- 
nation of these features that produces high helium-3 polarization and volume in the Hybrid Polarizer. With the scarcity and rising price of helium-3 [41], higher performance polarizers take on new importance for their ability to more efficiently use ${ }^{3} \mathrm{He}$. This chapter will first discuss the process of SEOP for producing hyperpolarized helium-3. I will then describe the design of a medical-imaging polarizer, with the specifics of the Hybrid Polarizer featured. The main components that improve helium polarization, the lasers and the alkali mixture, will be detailed in Sections 3.3 and 3.4. The helium-3 polarization measurement and calibration will be explained in Section 3.5, and the polarizer performance results are presented in Section 3.6.

\subsection{Spin-Exchange Optical Pumping}

In the process of spin-exchange optical pumping, hyperpolarized helium-3 is produced via spin-exchange collisions with optically pumped alkali metal atoms. Historically, the most common alkali metal used in SEOP was rubidium, which is ideal for several reasons: it has a wide energy spacing between the D1 and D2 transitions, it has a transition wavelength that can be easily reached by commerciallyavailable lasers, and it has a high vapor pressure among alkali metals. The rubidium and helium are placed in a glass cell with a small amount of nitrogen, and the total gas pressure in the cell can be up to $\sim 10 \mathrm{~atm}$. The cell is placed in an oven within a magnetic field, in our case $B_{0} \approx 20-30 \mathrm{G}$, and the oven is heated to vaporize the rubidium. Figure 3.1 illustrates the energy levels of rubidium- 87 . The hyperfine structure energy levels are the result of the coupling between the electron and nuclear magnetic moments, $\vec{F}=\vec{I}+\vec{J}$. In the presence of a magnetic field, the magnetic sublevels of the rubidium atom separate due to the Zeeman effect and are characterized by the quantum number $m_{F}$. Circularly polarized laser 


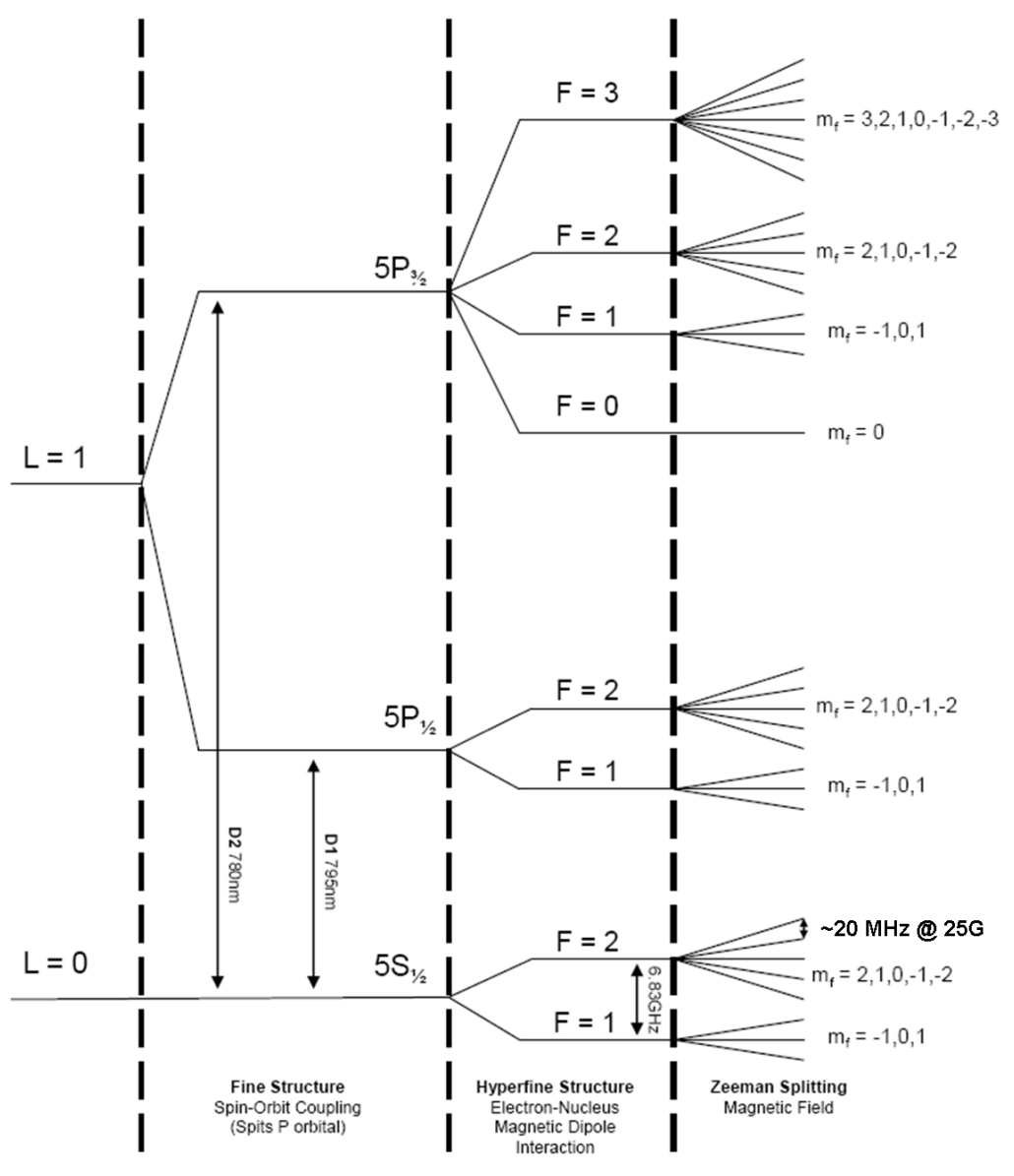

Figure 3.1: Energy levels of the $\mathrm{Rb}^{87}$ atom $(I=3 / 2)$ [42]. 
light is directed at the cell, tuned to the rubidium electron D1 transition.

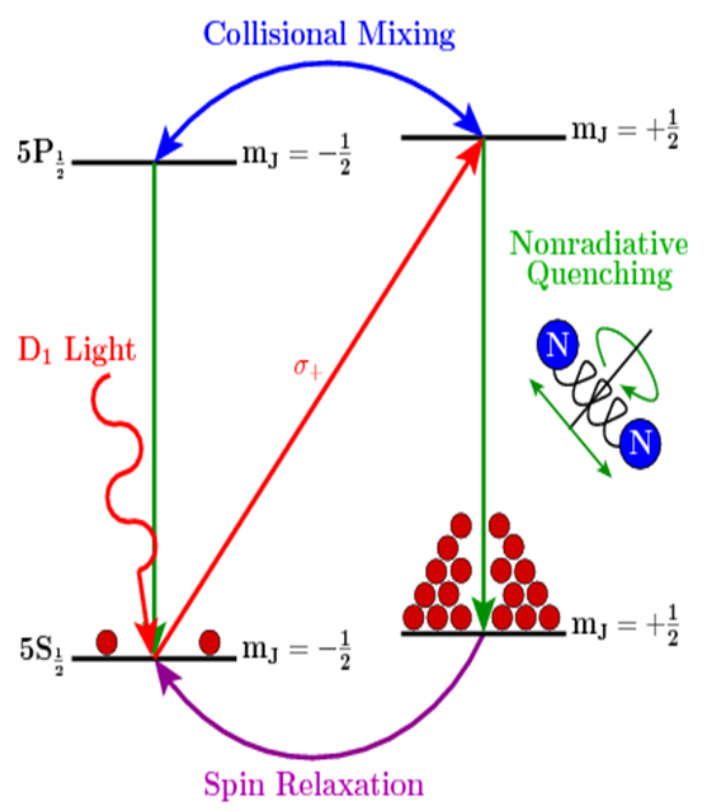

Figure 3.2: Optical Pumping of Rubidium [43]. Nuclear contributions are suppressed in this depiction.

The interaction between the incident light and the atom can be considered "sudden" with respect to the nuclear spin [44], and as such the hyperfine structure is not addressed in the following discussion. The incident light excites the electron from the $5 S$ ground state by transferring angular momentum to the valence electron as shown in Figure 3.2. This angular momentum transfer, $\Delta m_{J}= \pm 1$ where the sign is determined by the circular polarization of the laser, restricts the excitation to one of the ground state levels that are present in the magnetic field, here $m_{J}=-\frac{1}{2}$. From the $5 P_{\frac{1}{2}}$ excited state, decay back to the ground state is mediated by the addition of nitrogen gas which transfers the released energy into its rotational degrees of freedom, allowing for non-radiative quenching of the excited atoms [45]. This ensures there are no unpolarized photons in the cell. The system reaches an equilibrium with all of the rubidium atoms in the ground state which can not be excited by the incident light. Collisions between atoms result in some 
mixing of the $m_{J}$ levels in both the $5 P_{\frac{1}{2}}$ and the $5 S_{\frac{1}{2}}$ energy states, decreasing the equilibrium from a completely aligned state. Equilibrium polarization is reached in milliseconds at the temperatures and laser powers used in the Hybrid Polarizer. The saturation alkali polarization $P_{R b}$ is given by:

$$
P_{R b}(t \rightarrow \infty)=P_{\text {Circ }} * \cos \theta \frac{R_{o p}(\vec{r})}{R_{o p}(\vec{r})+\Gamma_{s d}},
$$

where $P_{\text {Circ }}$ is the circular polarization of the incident light, $\theta$ is the angle between the light and the magnetic holding field direction. The optical pumping rate $R_{o p}(\vec{r})$ is determined by the photon spectral flux density $\Phi(v, \vec{r})$ and the photon absorption cross section $\sigma(v)$ by:

$$
R_{o p}(\vec{r})=\int \Phi(v, \vec{r}) * \sigma(v) d v
$$

The spin destruction rate $\Gamma_{s d}$ is given by:

$$
\Gamma_{s d}=\sum_{N} \kappa_{s d}^{R b-N}[N]
$$

where the sum is over the rates due to each type of atom present, with density $[N]$, weighted by the associated spin-destruction rate constant $\kappa_{s d}^{R b-N}$. The key factors in equation 3.1 that can be adjusted to optimize $P_{R b}$ are the circular polarization of the laser, the laser power, and the photon absorption cross section. The photon absorption cross section can be adjusted by raising (lowering) the pressure of the gases in the cell to widen (narrow) the absorption peak.

Once the rubidium atom has been polarized, it is able to transfer that polarization to the helium-3 nucleus via binary collisions. The build up of helium polarization is a slow process, usually accomplished overnight for a medical imaging 
session the next day and is referred to as a "spinup". The helium-3 polarization $P_{\mathrm{He}}$ increases as:

$$
P_{\text {He }}(t)=P_{\text {HeSat }}\left(1-e^{-\frac{t}{T_{u p}}}\right),
$$

where $T_{u p}$ is defined here as the "spinup time constant", and $P_{\text {HeSat }}$ is the saturation ${ }^{3}$ He polarization given by:

$$
P_{\text {HeSat }}=P_{R b} * \frac{\gamma_{s e}}{\gamma_{s e}+\Gamma_{s d}}
$$

where $\gamma_{s e}$ is the spin-exchange rate:

$$
\gamma_{s e}=\kappa_{s e}[\mathrm{Rb}]
$$

and the spin-exchange rate constant for helium-rubidium has been measured to be $\kappa_{s e}=6.7 \pm 0.610^{-20} \mathrm{~cm}^{3} / \mathrm{s}$ [46]. At the operating temperatures of helium-3 SEOP polarizers utilizing rubidium alone $\left(T \sim 190{ }^{\circ} \mathrm{C}\right),[R b] \approx 6.2 * 10^{14}$ atoms $/ \mathrm{cm}^{3}$ and $\gamma_{s e} \approx \frac{1}{6.7}$ hours.

The spin-destruction rate $\Gamma_{s d}$ for helium-3 has contributions from collisions, field inhomogeneities, and wall relaxation:

$$
\Gamma_{\text {sd }}=\Gamma_{\text {collisions }}+\Gamma_{\text {field }}+\Gamma_{\text {wall }}
$$

$\Gamma_{s d}$ can be measured as a cell performance predictor by what is known as a "spindown", measuring the decay of helium-3 polarization at room temperature with the lasers turned off. From Equation 3.5, cells with smaller values of $\Gamma_{s d}$ will have higher helium-3 saturation polarizations. When seeking to improve the final helium-3 polarization in the SEOP process, one can either increase the spinexchange rate $\gamma_{s e}$, or decrease the spin-destruction rate, $\Gamma_{s d}$. In building the hybrid 
polarizer we focused on the former to improve ${ }^{3} \mathrm{He}$ polarization, in addition to increasing capacity and adding improved diagnostics.

\subsection{Hybrid Polarizer Design}

Systems for polarizing helium-3 with SEOP generally include a laser system, a polarization cell in an oven, a magnetic field, and an electronics system (Figure 3.3). When such systems are used for atomic or nuclear physics experiments, they typically include a sealed glass polarization cell $[47,48]$. For use in imaging, a polarizer needs a polarization cell which can dispense helium-3, and a gas handling system. The polarization cell used by the Hybrid Polarizer was a spherical glass cell $\sim 350 \mathrm{~mL}$ in volume made of alumina-silicate glass. The cell contained a small quantity of alkali metal, as well as helium gas which was replenished when necessary via a glass valve which extended out of the spherical cell connected by a narrow capillary. The cell was suspended in a ceramic oven with windows for light transmission. The oven was surrounded by several sets of coils- the main holding field coils, NMR drive coils, NMR pickup coils, and EPR drive coils as shown in Figure 3.4. On the Hybrid Polarizer there was also a set of counter-wound gradient coils that served to decrease observed "masing" effects which limited helium-3 polarization and are discussed in Appendix A.

Each time the hybrid polarizer was used for imaging, the helium-3/nitrogen mixture was added to the cell so that the pressure reached $\sim 120 \mathrm{psig}$. The oven was heated via forced air with two heaters (PureFlow PF-12 1200 W HotWatt, Danvers MA), and the holding field of $B_{\text {main }}=25 \mathrm{G}$ was supplied by a Helmholtz coil pair (Walker Scientific, Worchester MA) powered by a $36 \mathrm{~V}-12$ A power supply (BOP 36-12 Kepco, Flushing NY). The hybrid polarizer was equipped with four lasers (25 W Comet VBG-locked diodes Spectra-Physics, Santa Clara CA). Typical 


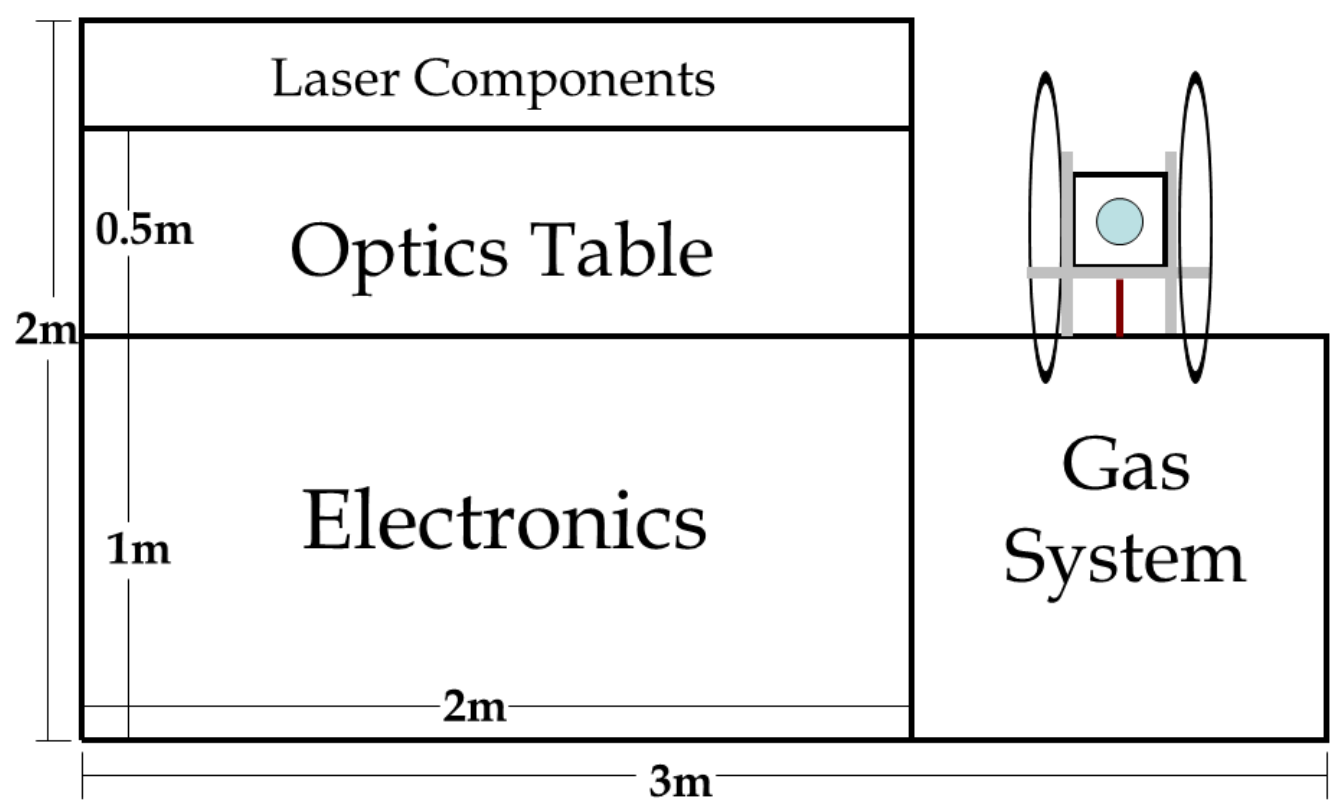

(a) Schematic

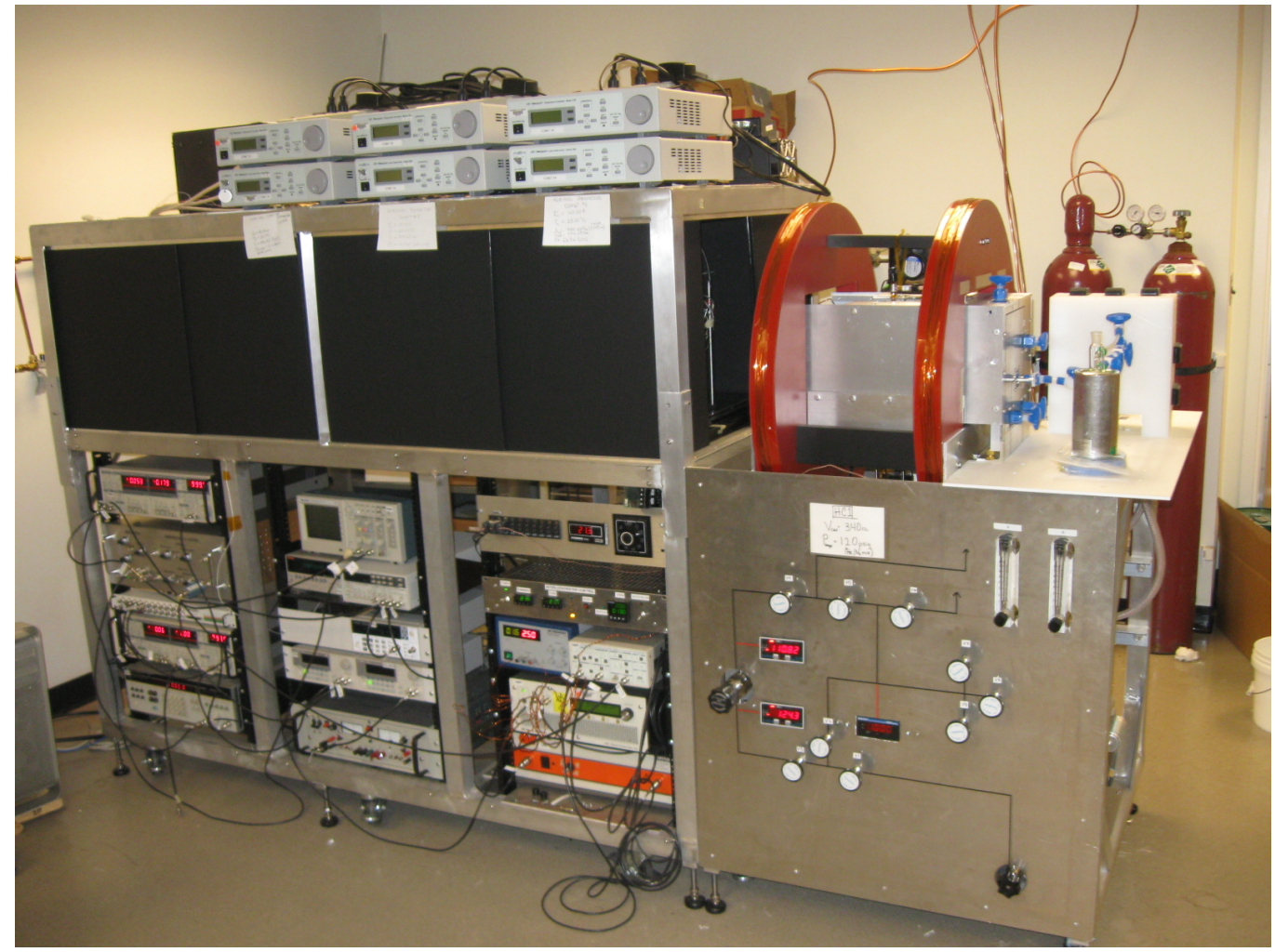

(b) Actual

Figure 3.3: Hybrid Polarizer. 


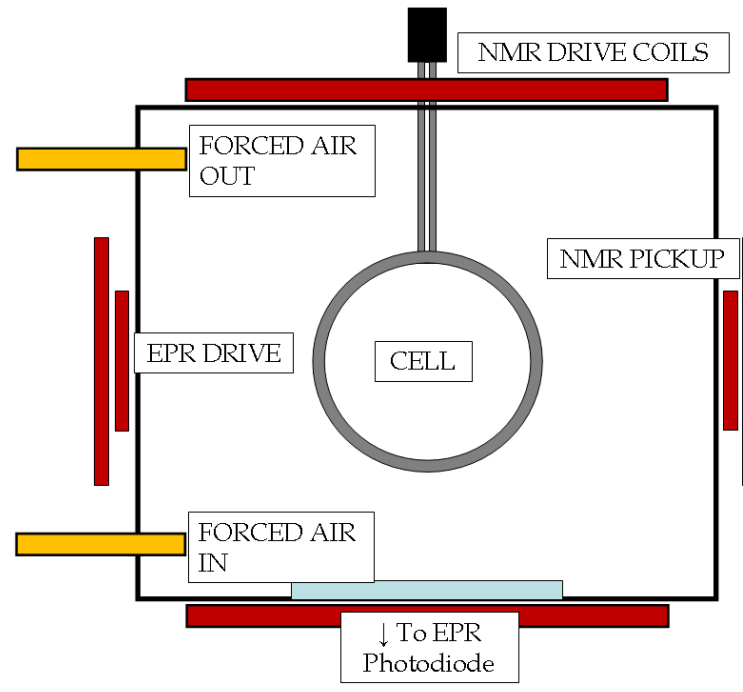

(a) Schematic

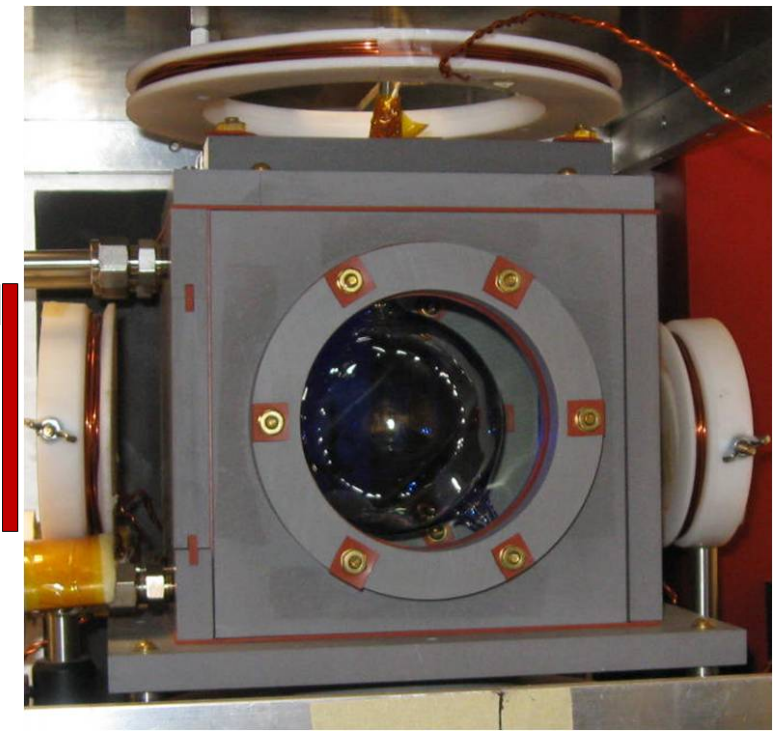

(b) Actual

Figure 3.4: Polarization cell in the ceramic oven. Various coils shown are used for monitoring the helium-3 polarization.

operation involved the use of three of these lasers to provide circularly polarized light. The specific lasers used were alternated to extend the lasers' lifetimes. They will be discussed in more detail in the next section.

The hybrid polarizer was equipped with a gas-handling system (Figure 3.5) that supplied the polarization cell with the appropriate helium-3 and nitrogen mixture for polarization, and provided a pathway for dispensing the helium for imaging. There were two main parts to the system: the fill manifold and the dispense manifold. The filling manifold connected the helium bottle to the cell through a regulator. The dispense manifold connected the cell to the vacuum pump, medical nitrogen, and the outlet.

Throughout the process of polarizing the helium-3, the signal was monitored using NMR as described in Section 3.5. After several hours of pumping, the oven was cooled using forced air at room temperature. To ensure that no alkali metal was removed from the cell, the cell valve was not opened until the oven had been 


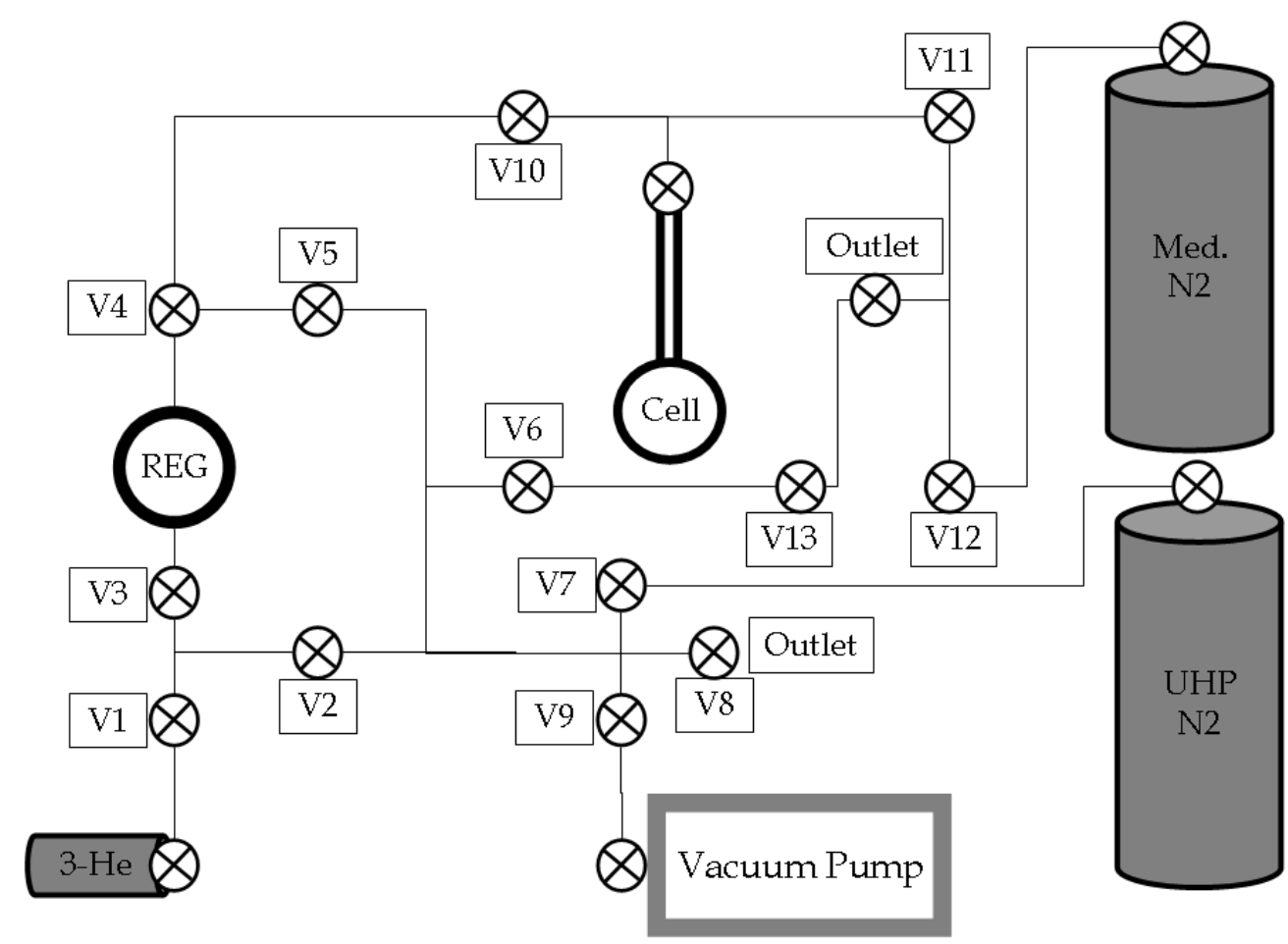

Figure 3.5: The gas system layout of the hyprid polarizer. The manifold consisted of stainless steel Swagelok tubes, fittings, and valves from valves V1-V9. The remaining components were plastic Swagelok tubes, fittings, and valves. Specific parts can be found listed in Appendix B.

below $40^{\circ} \mathrm{C}$ for 30 minutes. After leaving the dispense manifold, the helium-3 passed through a Teflon filter to ensure no alkali metal was being passed to subjects. The amount of gas dispensed was measured in a plastic syringe, and dispensed ${ }^{3} \mathrm{He}$ was mixed with medical-grade nitrogen when dosed to subjects for imaging to increase the total volume of gas inhaled. The helium polarization was measured in a separate calibration station to verify the hybrid polarizer calibration. ${ }^{3} \mathrm{He}$ quantities used in imaging varied based on polarization and imaging application from $50-500 \mathrm{~mL}$ and the total volume inhaled varied based on the subject and technique as well.

Several components of this design were improved based on the performance of 
the helium-3 target cells in the E02-013 experiment $\left(G_{E}^{n}\right.$ 2005-2006) [49] at Thomas Jefferson National Accelerator Facility (Newport News VA). As previously mentioned, the lasers and alkali metal mixture are features that enable the hybrid polarizer to produce highly polarized helium-3. In addition, the polarization cell capacity of 3L per batch was larger than the commercial helium-3 SEOP polarizer previously used at U.Va., which produced $\sim 1.6 \mathrm{~L}$ per batch. The optical setup of the Hybrid Polarizer was very different from previous helium-3 SEOP polarizers, in order to maximize efficient use of laser power. Lastly, measurement and calibration of the helium polarization were performed using Adiabatic Fast Passage NMR and Electron Paramagnetic Resonance frequency shift calibration. These techniques provided a helium polarization measurement that could be calibrated under various conditions in situ. All of these features will be outlined in more detail below.

\subsection{Lasers and Optics}

When previous helium-3 SEOP polarizers were designed, rubidium was chosen as the pumping alkali metal primarily because of its relatively low vapor pressure curve and transition properties which worked well with commercially available lasers. The most important change in the last ten years has been the availability of spectrally-narrowed lasers. The spectra of the newest diode lasers are an order of magnitude narrower, $0.2 \mathrm{~nm}$ compared to $2.0 \mathrm{~nm}$ on so-called broadband lasers (Figure 3.6). The narrowed spectra more closely match the rubidium absorption cross section, which is about $0.3 \mathrm{~nm}$ at operating temperature and cell pressure [43]. There is evidence in the literature that simply moving from broadband lasers to narrowed lasers can result in a $40 \%$ increase in ${ }^{3} \mathrm{He}$ polarization [50]. The lasers on the hybrid polarizer were Volume Bragg Grating-locked Comet line- 


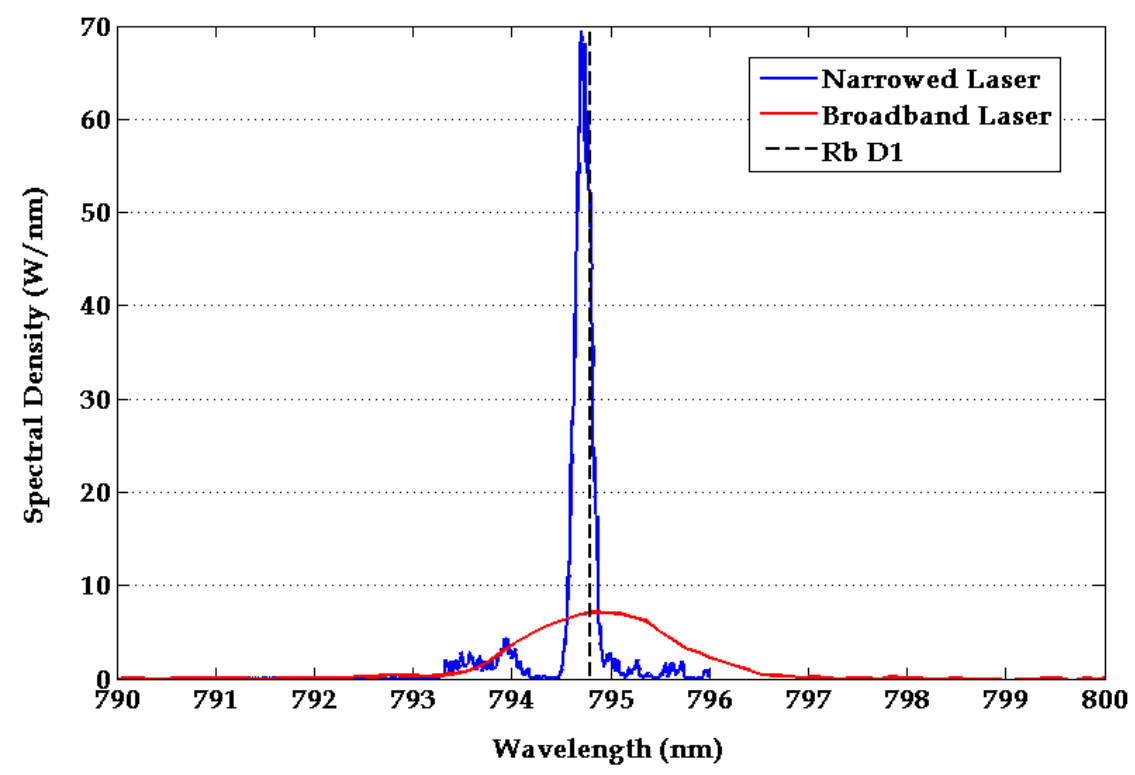

Figure 3.6: Spectral Density of narrowed lasers (blue) versus broadband diode lasers (red), with the rubidium D1 transition wavelength (black).

narrowed diode lasers centered at $794.8 \mathrm{~nm}$ with a linewidth of $0.2 \mathrm{~nm}$ (SpectraPhysics, Santa Clara CA). The central wavelength of the laser was determined by the diode current and temperature, and could be adjusted to match the rubidium D1 transition, which shifted to lower wavelengths when in the presence of highpressure helium.

The two functions of the optics setup shown in Figure 3.7 were to converted the linearly polarized light from the diodes to circularly polarized light for optical pumping, and to direct the light onto the polarization cell. Circular polarization was achieved through a linear beam-splitting cube and a quarterwave plate. The beam path was directed by the placement of mirrors and lenses. The lens placement in the Hybrid Polarizer serves to focus and overlap the beams from each laser on the center of the cell. The lasers are coupled together through a 5-to-1 fiber com- 


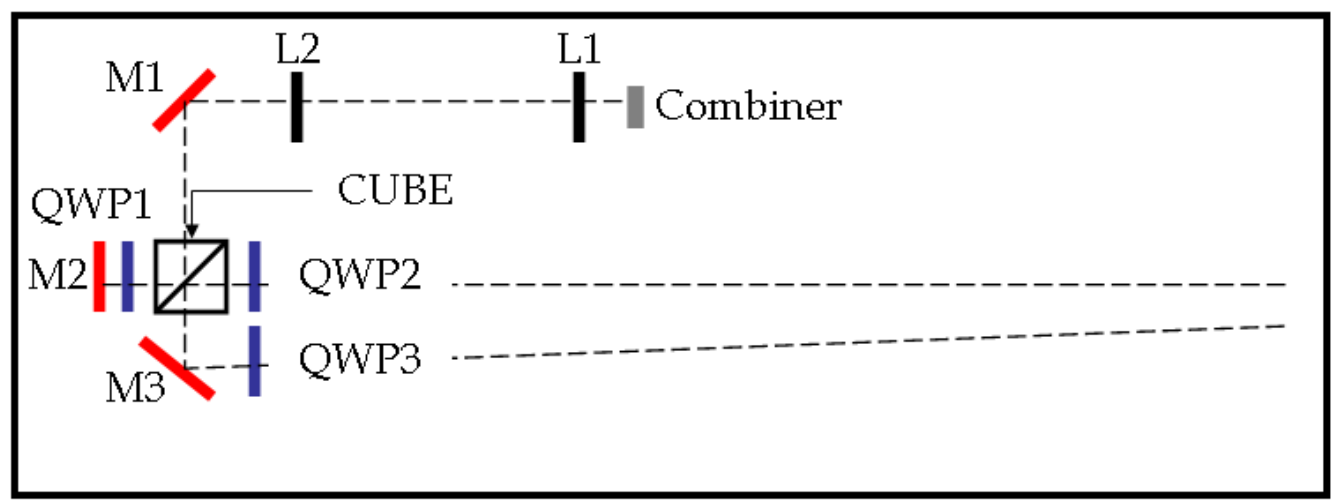

Figure 3.7: Hybrid polarizer optics layout (not to scale) includes three mirrors (M), a beam-splitting cube, three quarterwave plates (QWP), two lenses (L) and a fiveto-one fiber combiner.

biner (Avantes, Broomfield CO) which positions the individual laser fibers close to each other. Laser light exited the combiner as five distinct beams. Through the use of Fourier optics, the uniform angular distribution of the source beams enables their conversion into a spatial homogeneous beam at the center of the cell [51]. The location of the first lens L1 determines the beam size at the center of the cell, and the second lens (L2) controls the overlap of the beams. This technique allows optical coverage of the cell that is far more homogeneous than simply using the output of the fiber combiner unchanged, as demonstrated in Gaussian beam simulations shown in Figure 3.8. 


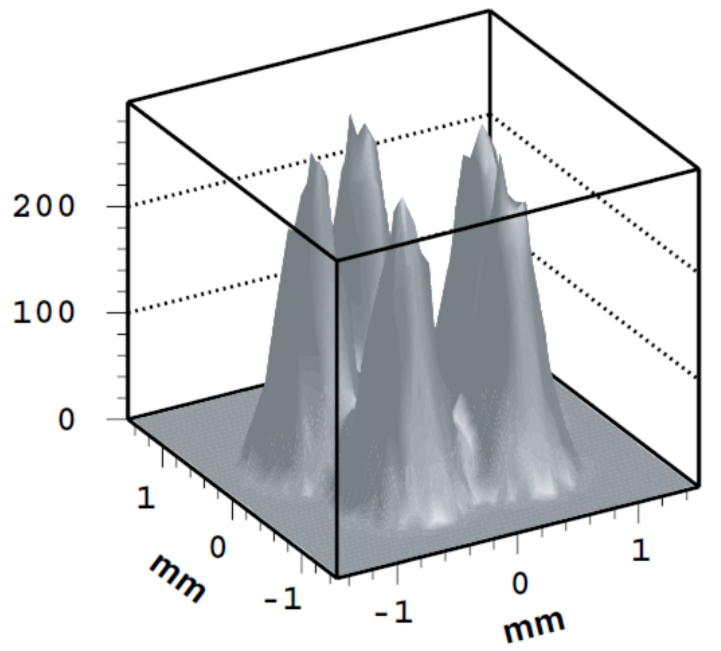

(a) Beam Intensity Profile at the first lens

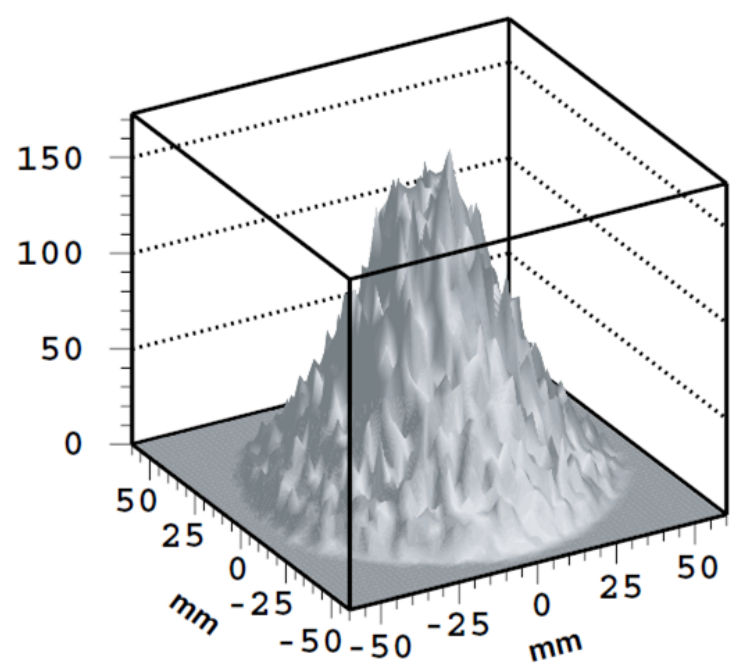

(b) Beam Intensity Profile at the center of the cell

Figure 3.8: Simulations showing the profiles of the beams at (a) L1 and (b) the center of the cell [52]. 


\subsection{Hybrid SEOP}

In Hybrid Spin-Exchange Optical Pumping, potassium is added to the rubidium in the polarization cell to increase the efficiency of transferring polarization to the helium-3 [53]. Whereas only $\sim 2 \%$ of the spin of a rubidium atom is ultimately transferred to the helium-3 nuclei, the spin exchange efficiency approaches $25 \%$ for potassium and helium-3 [54]. An important feature of hybrid SEOP is that alkali metals readily spin exchange with each other so the potassium polarization will be nearly equal to that of the rubidium. By using a mixture that is rich in potassium and lean in rubidium, the same lasers used in rubidium-only SEOP can be used, but with a much higher spin-exchange efficiency for polarizing helium-3 (Figure 3.9).

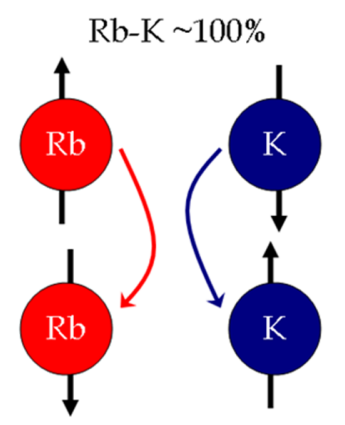

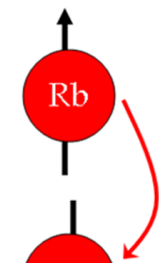
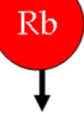

$\mathrm{Rb}-3 \mathrm{He} \sim 2 \%$
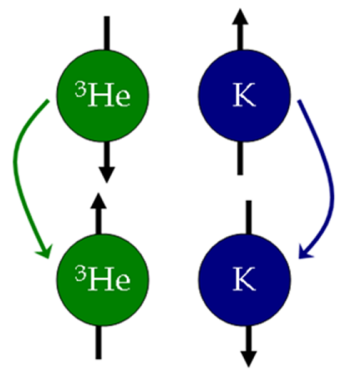

$\mathrm{K}-3 \mathrm{He} \sim 25 \%$

Figure 3.9: Hybrid spin-exchange between helium-3 and rubidium or potassium.

The ratio of potassium to rubidium in the alkali mixture can have a great effect on how much advantage is gained from hybrid pumping. When the hybrid mix- 
ture contains too large an amount of potassium, there is not enough rubidium to be pumped by the lasers and keep the alkali polarization high. If the mixture contains too little potassium the extra efficiency does not significantly impact the final helium-3 polarization. Studies performed by our group have produced a guideline that the optimal ratio of potassium to rubidium for helium-3 polarization is $\sim 6 / 1$ at operating temperature, but that strict control is not necessary as substantial improvement is attained over a relatively wide range of mixtures [43].

For a traditional rubidium SEOP polarizer the cell is usually held at $\sim 190^{\circ} \mathrm{C}$ during pumping, which gives a rubidium density of $[\mathrm{Rb}] \sim 10^{15}$ atoms $/ \mathrm{cm}^{3}$. Due to the higher vapor pressure of potassium, the oven temperature for hybrid SEOP should be increased, in our conditions to $240^{\circ} \mathrm{C}$ [42]. Finding the optimal set temperature was part of the commissioning process covered in Section 3.6.

\subsection{Measurement and Calibration}

The helium polarization in the cell is monitored periodically throughout the spinup using NMR. The Hybrid Polarizer used Adiabatic Fast Passage (AFP) NMR [55] due to the large signal-to-noise ratio in this method, and calibrated the NMR measurement using Electron Paramagnetic Resonance (EPR) [56].

\subsubsection{AFP}

In Adiabatic Fast Passage, the helium-3 spins are flipped $180^{\circ}$ and back during the course of one measurement. On the Hybrid Polarizer, the spin flip was accomplished by applying a transverse RF field $B_{1}$ at a frequency $\omega_{0}$ which is slightly above the Larmor frequency of the holding field $B_{\text {Hold }}=25 \mathrm{G}$. The holding field was increased past the resonance field $B_{0}$ and then ramped back down to the orig- 
inal level. The effective magnetic field in the rotating frame of the helium-3 nuclei becomes:

$$
\vec{B}_{e f f}=\left[B_{\text {main }}(t)-B_{0}\right] \hat{z}+B_{1}\left(\omega_{0}\right) \hat{x}^{\prime},
$$

where $B_{\text {main }}(t)$ described the field sweep. A set of perpendicular "pickup" coils detected the signal in the transverse plane increased as the spins go past, resulting in two peaks as the spins flip over and flip back. Figure 3.10 displays an AFP measurement trace from the Hybrid Polarizer. The red line indicates the value of the holding field, and the blue curve shows the signal detected by the pickup coils.

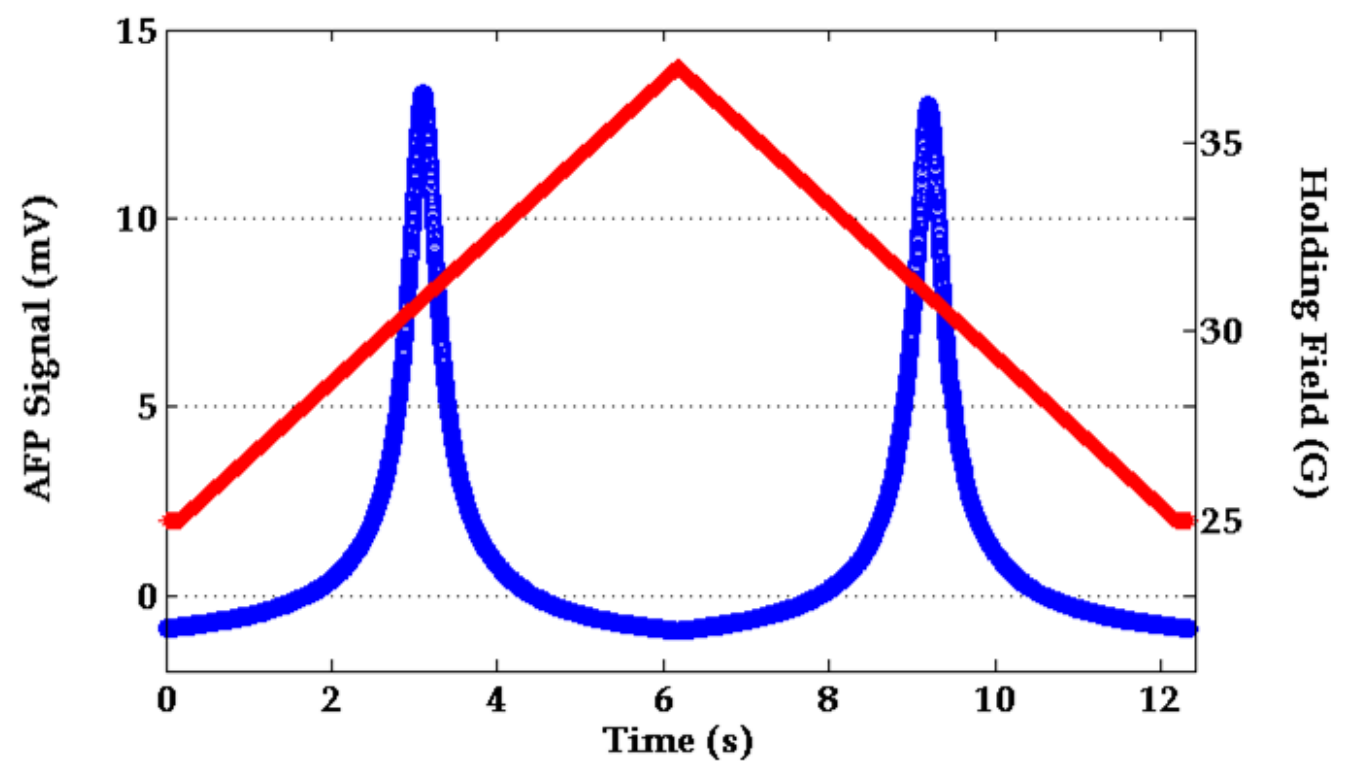

Figure 3.10: Sample AFP trace: The ${ }^{3} \mathrm{He}$ transverse magnetization signal is shown in blue, and the holding field magnitude is shown in red.

The name Adiabatic Fast Passage derives from two competing timing requirements which serve to minimize polarization loss during a measurement. In order for the field sweep to be considered adiabatic, the main field must not change 
much per revolution of the helium-3 spins so that the spins can follow it:

$$
\frac{\dot{B}_{\text {main }}}{B_{1}} \ll \omega_{0} .
$$

The fast requirement of the field sweep timing relates the changing magnetic field to the spin-relaxation rate. Magnetic field inhomogeneities lead to spin relaxation, and the relative size of inhomogeneities is largest when the effective magnetic field is the smallest, when $B_{\operatorname{main}}(t)=B_{0}$. The longitudinal spin-relaxation rate in the rotating frame depends on the size of the inhomogeneities compared to the transverse RF field, and how quickly the gas explores the area as follows [57]:

$$
\frac{1}{T_{1_{r}}}=D \frac{\left|\nabla B_{z}\right|^{2}}{B_{1}^{2}} \ll \frac{\dot{B}_{\text {main }}}{B_{1}},
$$

where $T_{1}$ is the spin-relaxation time constant, $D$ is the gas diffusivity, and $\nabla B_{z}$ is the gradient of $B_{z}$. When the sweep parameters are chosen to meet both of the above conditions,

$$
D \frac{\left|\nabla B_{z}\right|^{2}}{B_{1}^{2}} \ll \frac{\dot{B}_{\text {main }}}{B_{1}} \ll \omega_{0},
$$

the AFP NMR process can result in a minimal polarization loss per measurement.

The diffusion coefficient and field variation are fixed by the system, but the other parameters are can be varied. In the use of the Hybrid Polarizer, the AFP condition was satisfied by the following values

$$
\begin{array}{r}
D \approx 0.2 \mathrm{~cm}^{2} / \mathrm{s} \quad \nabla B_{z}^{2} \approx\left(\frac{10 \mathrm{mG}}{\mathrm{cm}}\right)^{2} \\
B_{1} \approx 100 \mathrm{mG} \quad \dot{B}_{\text {main }}=\frac{10 \mathrm{G}}{6 \mathrm{~s}} \quad \omega_{0}=2 \pi * 100 \mathrm{kHz} \\
0.002 \mathrm{~Hz} \ll 16.67 \mathrm{~Hz} \ll 2 \pi * 100 \mathrm{kHz}
\end{array}
$$


We measured helium-3 signal losses of $1 \%$ per measurement in the initial tests of the Hybrid Polarizer, but those losses increased over the course of the experiments in this work. These results are discussed in more detail in Section 3.6.

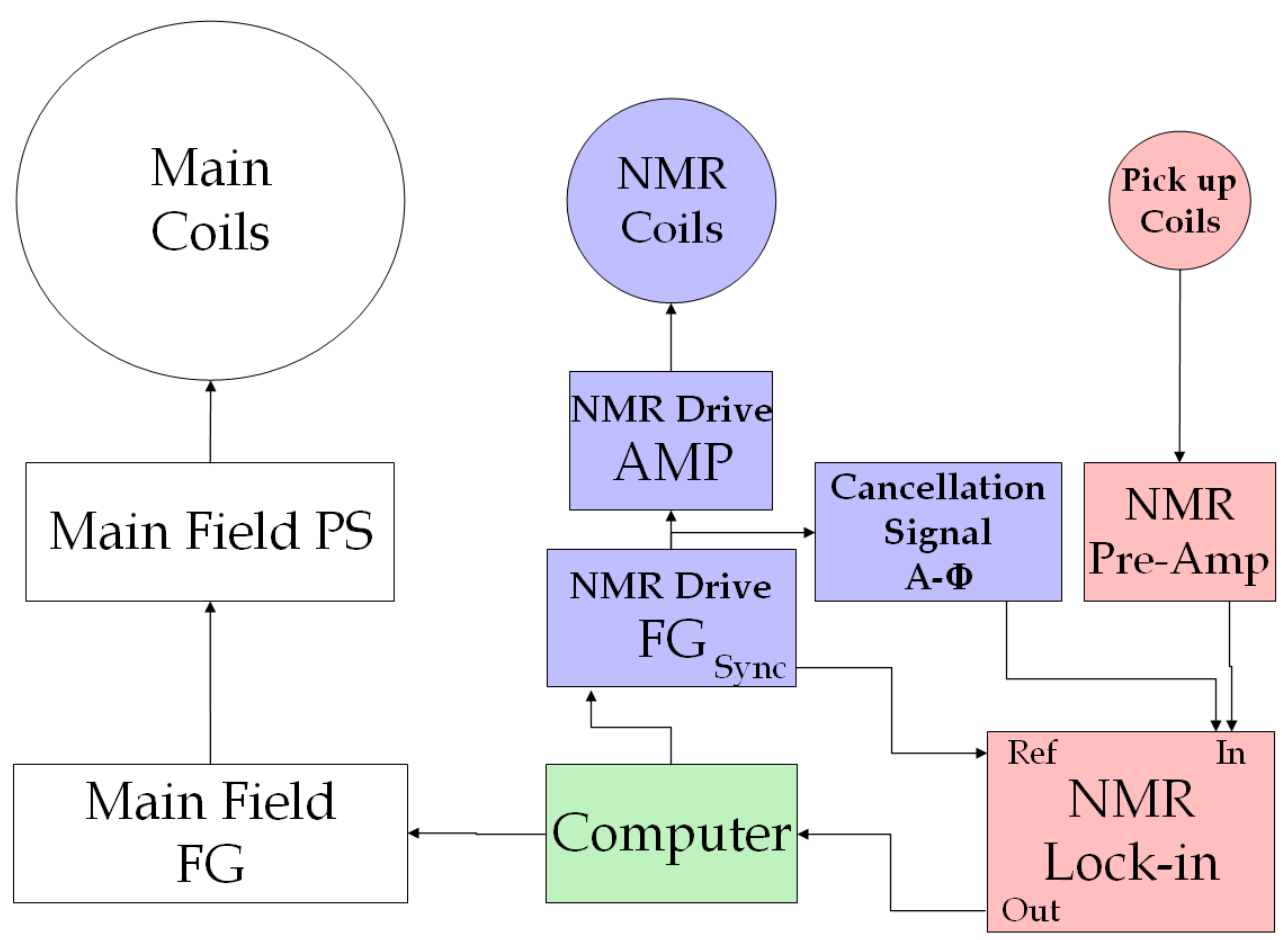

Figure 3.11: Electronics used to make an NMR AFP measurement. A function generator (FG) controls the main field sweep input to the power supply (PS). The transverse field is controlled by the NMR Drive FG and amplifier. The signal from the pick up coils is amplified before being combined with an optional cancellation signal at the lock-in amplifier. Specific model numbers and settings can be found listed in Appendix B.

The timing and execution of an AFP NMR measurement was controlled by a LabVIEW program (National Instruments, Austin TX). The electronics required are illustrated in Figure 3.11. A complete listing of model numbers and settings is provided in Appendix B. The measurement began with the main field at its default value $B_{\text {Hold }}=25 \mathrm{G}$. Referring to labels in Figure 3.11, first the NMR drive function generator (FG) was activated, then the main field FG was triggered to sweep 
up through resonance and back down, $B_{\text {main }}(t)=25 \rightleftarrows 35 \mathrm{G}$. The signal from the pickup coils was sent to a pre-amp before being sent to lock-in amplifier. An additional input to the lock-in was used as a background cancellation tool. A sine wave signal at the NMR drive frequency with a variable amplitude and phase was generated by a home-built A- $\Phi$ box. This source was used to cancel any spurious signal received by the pickup coils due to imperfect alignment. Without this background cancellation signal, the AFP trace would sit on top of a background that could be eliminated in data analysis, but might lead to amplifier saturation.

The signal peaks from the up and down legs of the holding field sweep (Figure 3.10) were each fit to a Lorentzian function:

$$
y=A \frac{w}{\sqrt{\left(x-x_{0}\right)^{2}+w^{2}}},
$$

where $A$ is the peak amplitude parameter, $w$ is the peak width, and $x_{0}$ is the peak center. The amplitude fit parameter was plotted against time to produce a spinup plot as shown in Figure 3.12. Measurements were made more frequently early in the pumping, when the polarization was changing most rapidly with time. As the signal increased, the points were taken further apart in time. The spinup curve was fit to:

$$
R(t)=R_{\max } *\left(1-e^{-\frac{t-t_{0}}{T_{u p}}}\right),
$$

where $R_{\max }$ is the saturation amplitude, $t_{0}$ is the time offset from zero polarization, and $T_{u p}$ is the spinup time constant. The values of $R_{\max }$ and $T_{u p}$ were used to characterize cells and optimize performance, but it is useful to convert $R_{\max }$ to absolute helium polarization to compare performance under different conditions. 


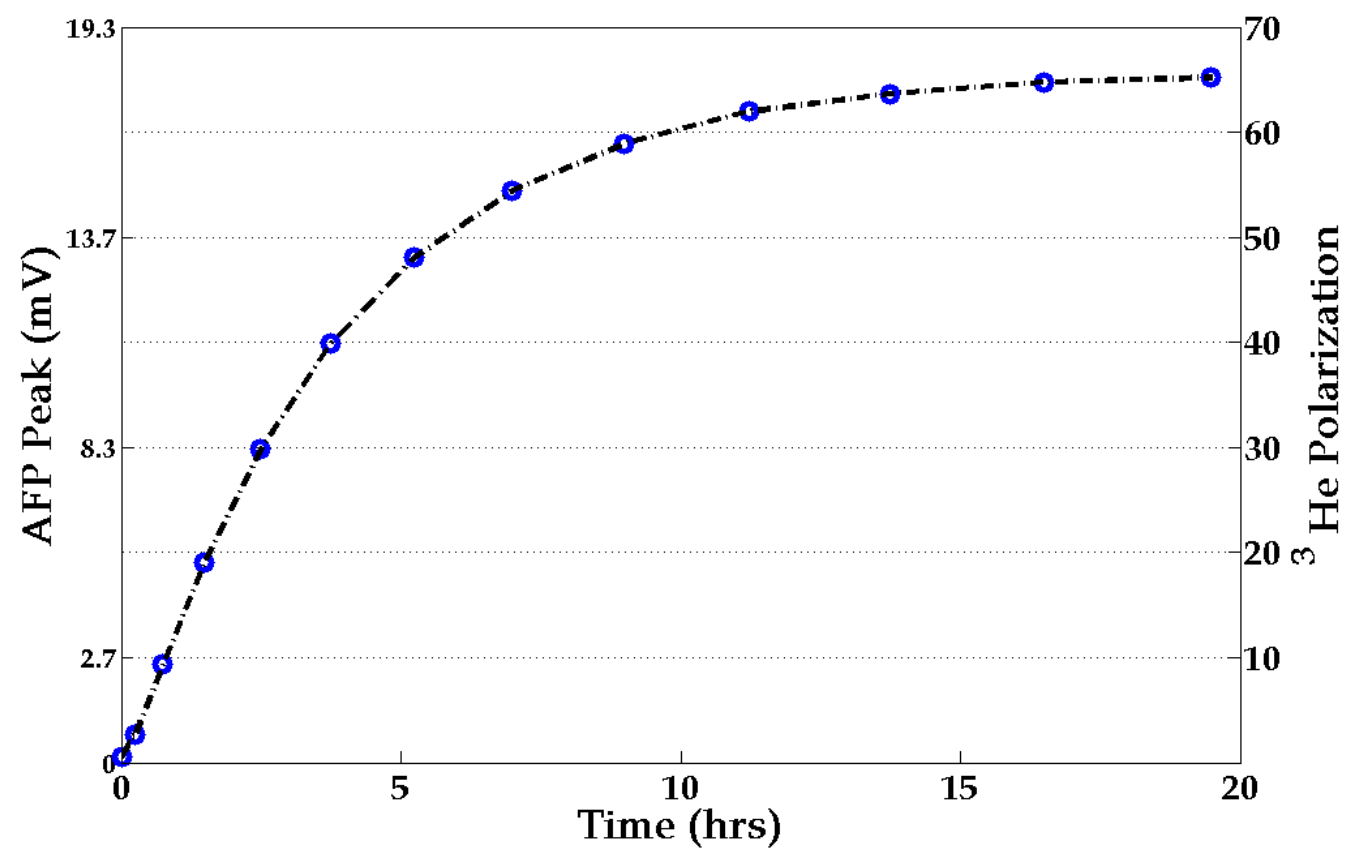

Figure 3.12: A calibrated helium-3 spinup from 08/10/2010: $T_{\text {oven }}=230^{\circ} \mathrm{C}, P_{\text {cell }}=$ 120 psig, 3 COMET Lasers. Spinup fit parameters: $R_{\text {sat }}=18.18 \mathrm{mV}, P_{\text {sat }}=65.4 \%$, $T_{\text {up }}=3.9 \mathrm{hrs}$.

\subsubsection{EPR}

A calibration technique based on Electron Paramagnetic Resonance (EPR) is used in the Hybrid Polarizer to convert an AFP peak amplitude to an absolute helium-3 polarization. EPR calibration involves monitoring the alkali metal electron transition frequency between Zeeman levels under two different magnetic field conditions. The net magnetization of the helium-3 nuclei produces its own magnetic field which can add to or subtract from the main holding field. A change in the magnetic field alters the resonant frequency of the alkali metal valence electrons' Zeeman transitions (figure 3.1). The spacing between the $m_{F}$ levels varies approximately linearly with field at the low magnitude of the Hybrid Polarizer's holding field, $B_{0}=25 \mathrm{G}$, but our calibration also accounts for quadratic terms [58]. The largest portion of the helium-generated field's effect on the alkali metal frequency comes from alkali- ${ }^{3} \mathrm{He}$ collisions, with a smaller portion due to long-range 
interactions of the gas [59], [60]. The calibration is performed by monitoring this frequency while the direction of the helium-3 field is reversed. The change in frequency is proportional to the field generated by the helium magnetization. This field magnitude can be converted to an absolute ${ }^{3} \mathrm{He}$ polarization.

The EPR calibration method takes advantage of the fact that even with the addition of nitrogen as a buffer, some of the alkali electrons in the excited states decay via photon emission. Due to collisional mixing, both D1 and D2 photons are produced [61]. The rubidium D2 photons are monitored during an EPR measurement, to avoid the large background created by the scattering of the pump beam at the D1 wavelength. During steady state pumping the intensity of photon emission is relatively low, as most of the atoms are in the outer most $m_{F}$ level. Upon the application of a resonant field, electrons are driven to adjacent levels which enables them to be pumped again by the laser. This results in an increase in D2 fluorescence. The light passes through a window on the bottom of the oven and is collected by a photodiode mounted under the oven, which has an optical filter to select the D2 wavelength and block the pump light background.

The electronics setup for an EPR measurement is illustrated in Figure 3.13, and a complete listing of model numbers and settings is provided in Appendix B. To begin the EPR calibration procedure an RF field was generated from the EPR voltagecontrolled oscillator (VCO). The output of the EPR VCO was modulated at a low frequency $\sim 200 \mathrm{~Hz}$ by the EPR MOD FG, which also served as a reference for the lock-in amplifier receiving the photodiode signal. The alkali transition frequency was determined via a frequency-modulated (FM) sweep, in which the frequency of the transverse field was incrementally increased and the photodiode output was recorded at each step. The resulting lock-in output signal is shown in Figure 3.14. The trace due to an FM sweep is the derivative of the intensity of the light, so it 


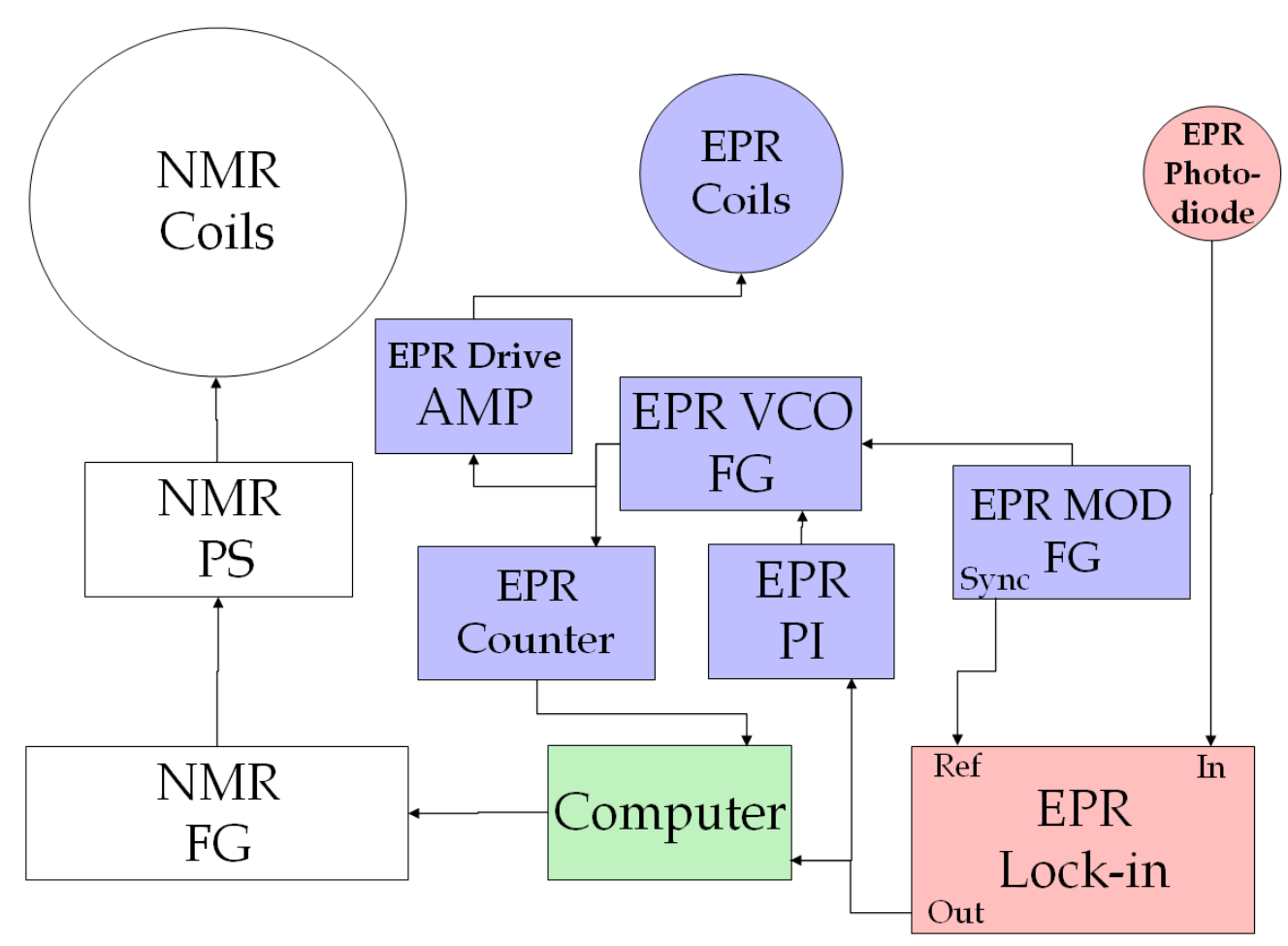

Figure 3.13: EPR electronics setup on the hybrid polarizer. The EPR VoltageControlled Oscillator function generator (FG) is used to generate the field to drive alkali electron transitions. The output of the EPR VCO FG is modulated by the MOD FG which allows the photodiode signal to be easier to detect isolate from the laser background. The transition frequency of the VCO is determined by a frequency sweep. During the measurement, the photodiode signal drives a proportional integration (PI) circuit which changes the VCO frequency as the helium-3 spins are flipped by the NMR drive.

goes through zero at resonance [54,62].

The EPR transition occurs at a slightly different frequency for each metal species present in the alkali mixture, as indicated by the multiple peaks present in Figure 3.14. This signal was generated measuring the intensity of rubidium D2 light, but the transitions were induced by depolarizing different alkali species their unique frequencies. The species that are present in the hybrid metal mixture are rubidium87 (peak labeled A), rubidium-85 (not pictured), potassium-39 (large peak), and potassium-41 (peak labeled B). EPR measurements on the Hybrid Polarizer were 


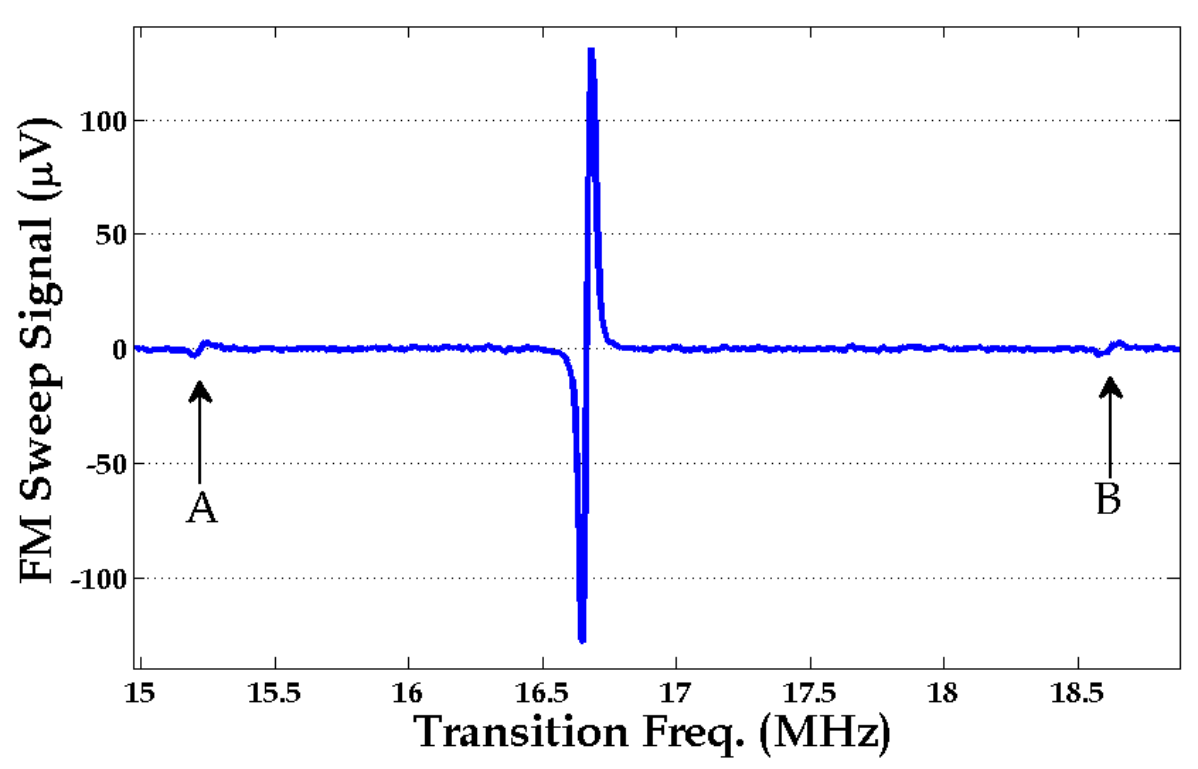

Figure 3.14: An EPR FM Sweep trace. The frequency of the RF field was increased, and the photodiode signal recorded at each step. The amplitude of the lock-in signal crosses zero as the D2 fluorescense reaches a maximum. There are spikes representing transitions of each of the alkali species in the cell: ${ }^{39} \mathrm{~K}$ (large peak), ${ }^{41} \mathrm{~K}(\mathrm{~A})$, and ${ }^{87} \mathrm{Rb}(\mathrm{B})$.

made by inducing transitions in potassium-39, as it produced the largest signal. The exact transition frequency was determined by fitting the trace to a line as it crosses through zero.

To make a calibration measurement, the EPR VCO was set to the measured transition frequency. The lock-in output was sent to a Proportional-Integration (PI) Feedback circuit [63]. When activated by a switch, the PI circuit sent a signal to the VCO control input which adjusted the center frequency of the EPR drive to keep the lock-in signal at zero. As the effective field experienced by the alkali metal changed, the EPR drive field was no longer resonant and the lock-in signal increased/decreased. The PI circuit forced the EPR drive FG to change its central frequency so that the signal went back to zero. A frequency counter was used to track the changing central frequency of the VCO during the measurement.

Once the VCO frequency had been set, the calibration procedure was as fol- 
lows:

1. Take an AFP measurement.

2. Turn on the EPR drive field, begin monitoring the EPR frequency and activate the feedback circuit via a switch.

3. Turn on the NMR drive and flip the ${ }^{3} \mathrm{He}$ spins by sweeping the frequency through resonance (keeping the main field constant).

4. Turn off the NMR drive and monitor the new frequency.

5. Turn on the NMR drive and flip the spins back with another frequency sweep.

6. Turn off the NMR drive and monitor the final frequency.

7. Turn off the EPR drive field.

8. Take another AFP measurement.

Figure 3.15 displays a representative EPR measurement. The black arrows represent the direction of the holding field, and the red arrows represent the direction of the magnetic field due to the polarized helium-3. Frequency data from each segment was averaged to calculate a change in frequency.

The equations used for converting the change in frequency to a magnetic field strength are detailed in Appendix B. Once the helium-3 magnetic field strength $B_{H e}$ had been calculated, it was directly converted into a helium-3 polarization $P_{H e}$ using the formula:

$$
P_{\mathrm{He}}=\frac{2}{\kappa_{0} *\left[{ }^{3} \mathrm{He}\right]} * \frac{3 * B_{\mathrm{He}}}{2 \mu_{0}},
$$

where $\mu_{0}$ is the permeability of free space, $\left[{ }^{3} \mathrm{He}\right]$ is the density of helium-3, and $\kappa_{0}$ 


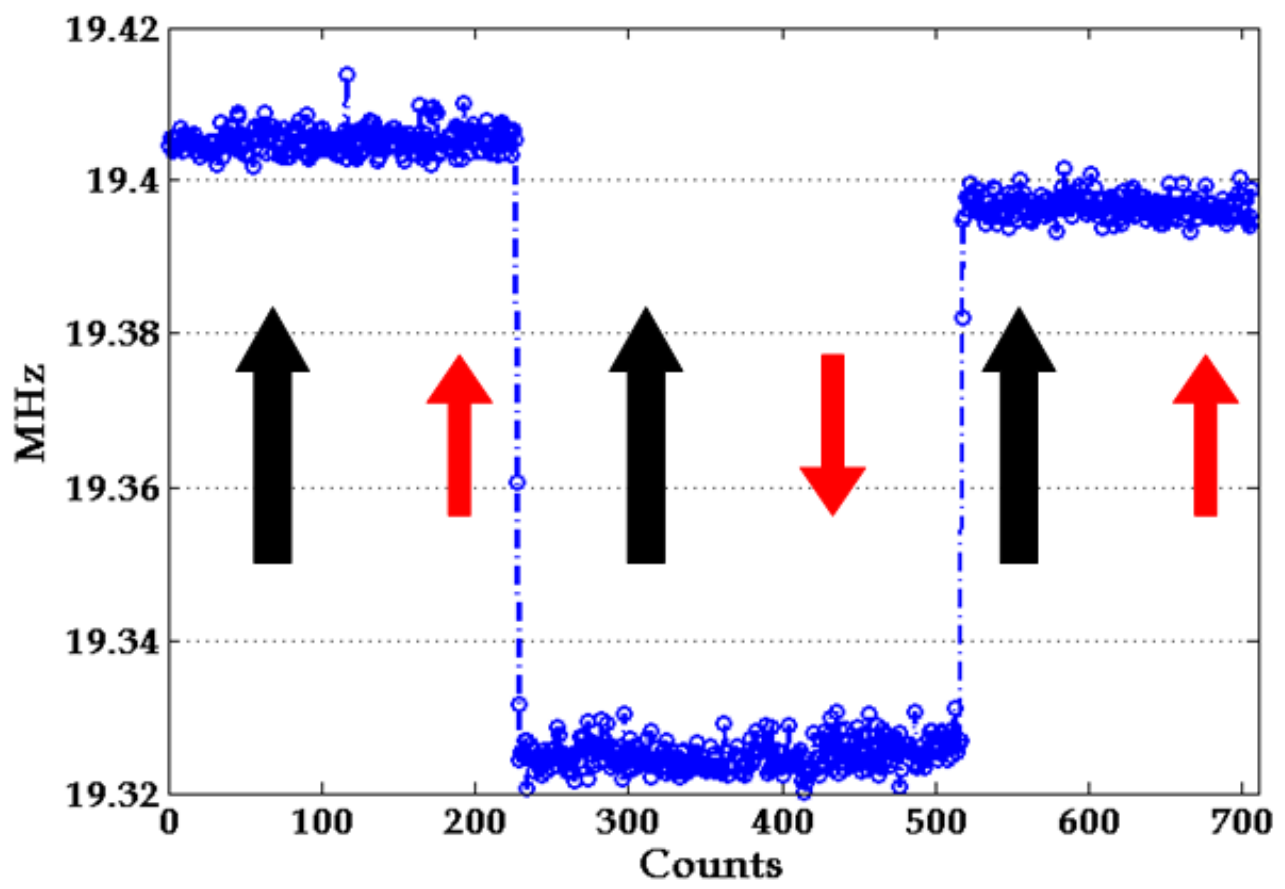

Figure 3.15: EPR frequency counter measurement while performing a calibration measurement. The black arrow indicates the direction of the main field, and the red arrow indicates the direction of the field induced by polarized ${ }^{3} \mathrm{He}$.

is the empirical shift constant:

$$
\kappa_{0}=5.99+0.0086\left(T-200{ }^{\circ} \mathrm{C}\right),
$$

and $\mathrm{T}$ is the polarization cell temperature in Celsius. Once the polarization was measured, it was used to calibrate the AFP measurements taken immediately before and after the measurement. There were helium-3 polarization losses associated with each of the frequency-sweep spin flips, as well as during the period the spins remain flipped. Each AFP peak height was matched with its corresponding frequency change, and the average calibration constant was used. This method of calibration of the helium-3 polarization could be repeated easily as new operating parameters were used, such as oven temperature and cell pressure. 


\subsection{Optimization and Performance}

In order to determine the peak performance of the hybrid polarizer, spinups were performed using three lasers with the oven at different set temperatures. Metrics such as saturation polarization and spinup time constant from the spinup fit were calculated, but for optimization we looked at the helium-3 polarization after $\sim 15$ hours of pumping. This parameter, $P_{15}$, was relevant due to the manner in which the Hybrid Polarizer was commonly used for medical imaging, with the cell pumped overnight for a study in the morning. We also measured the helium-3 polarization after five hours of pumping, $P_{5}$, for information about performance of the polarizer for the situation in which a second imaging session was planned for the same day. The results of these temperature tests are presented in Figure 3.16. The optimum oven set temperature for Hybrid Cell 1 (HC1) with three lasers was $T_{\text {oven }}=225^{\circ} \mathrm{C}$. In the shortened spinup case, we found that it was best to use the highest oven temperature $T_{\text {oven }}=240{ }^{\circ} \mathrm{C}$, which gave the fastest spinup rate.
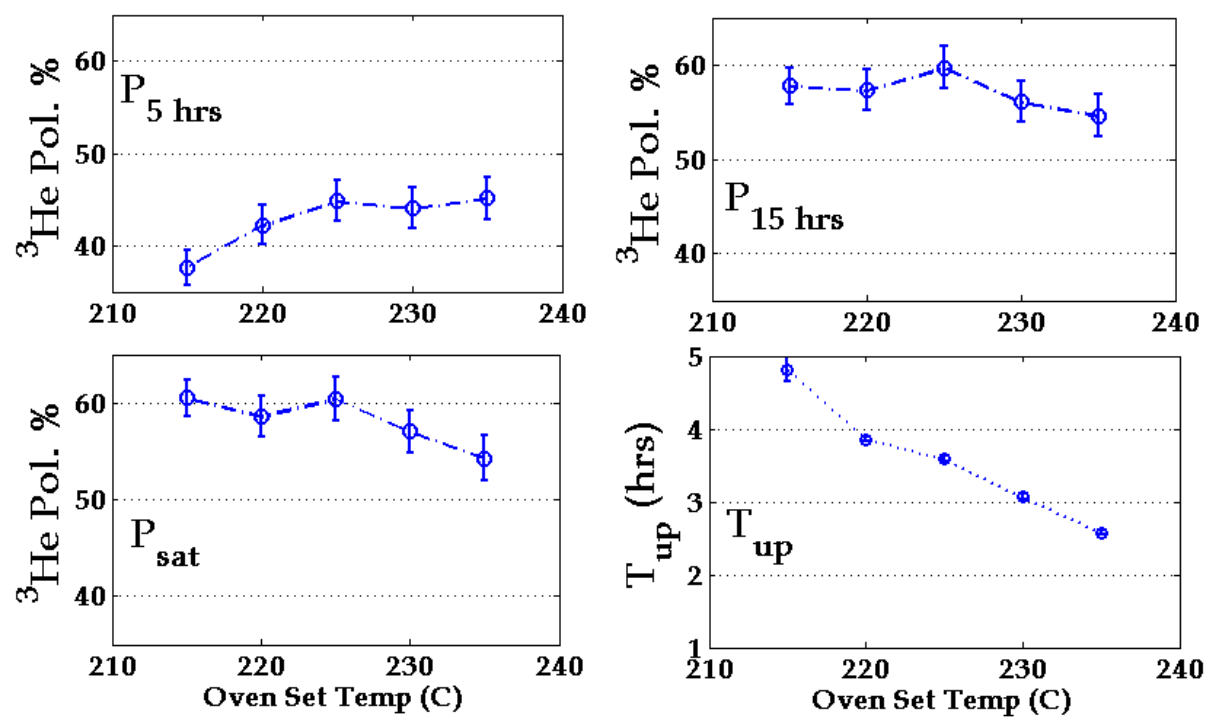

Figure 3.16: Temperature optimization results for Hybrid Cell 1 with three lasers. Helium-3 polarization after five and 15 hours, as well as fit parameters $P_{\text {sat }}$ and $T_{u p}$ are plotted versus oven set temperature. 
Over the last three years, the hybrid polarizer has been used to provide gas for human, animal, and phantom helium-3 MR studies. Gas dispensed was consistently measured to have ${ }^{3} \mathrm{He}$ polarization above $50 \%$, but there has been a noticeable decline from the initial performance as illustrated in Figure 3.17a. In addition to the polarization decline, the losses per AFP measurement increased from 1.3\% to $7 \%$, and the EPR FM sweep amplitude decreased by a factor of 100 , which made calibration difficult. These effects are illustrated in Figure 3.18.

Starting in April-May 2011, we reduced the frequency with which AFP measurements were made once the signal neared saturation, to reduce polarization loss due to the measurements. The polarization measurements on Figure 3.17a from that period and beyond represent the final point acquired during the spinup, often 2-3 hours before pumping was stopped. Independent measurements of dispensed gas polarization lead us to the conclusion that the hybrid polarizer was consistently reaching $\sim 50 \%$ helium-3 polarization with $\mathrm{HC} 1$, but not much higher. The gradual decline in performance led us to change the cell in June 2011.

Upon removing $\mathrm{HC} 1$ from the hybrid polarizer, we observed that the alkali metal had formed a large pool on the bottom and sides of the polarization cell throughout the course of its use (Figure 3.19a). We believe that some of the performance loss was due to this alkali pool. The metal obscured the cell from the EPR photodiode and possibly shielded the cell during AFP measurements. With the installation of the next cell Hybrid Cell 2 (HC2), the hybrid polarizer was once again be able to produce $>60 \%$ polarized ${ }^{3} \mathrm{He}$, as shown in Figure $3.17 \mathrm{~b}$. AFP losses decreased with the new cell, and the EPR FM sweep amplitudes recovered to approximately the same level as the initial measurements of HC1. It should be noted that at the same time as the cell change we began using all four available lasers to polarize, as performance of the lasers decreased over time as well. 


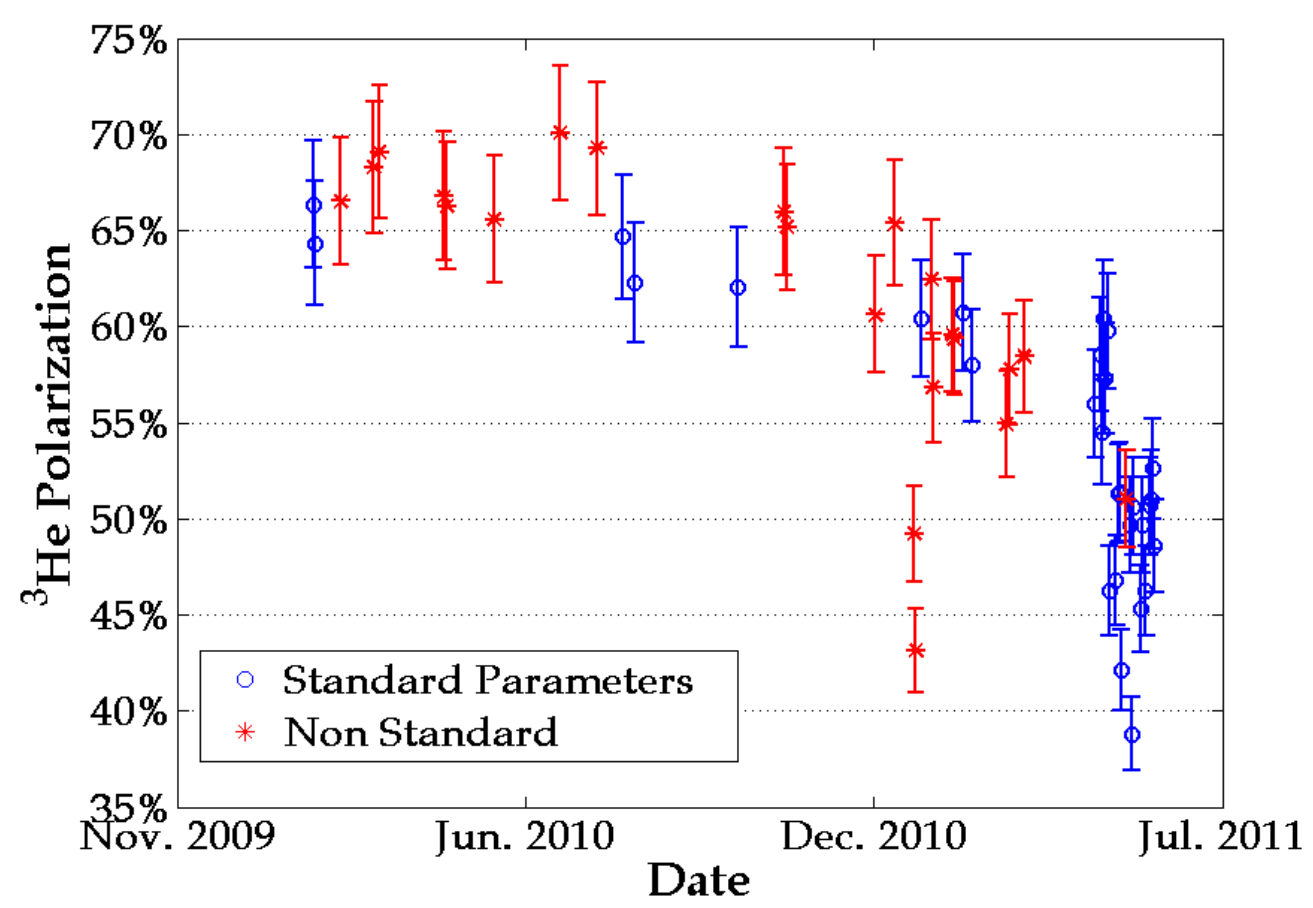

(a) HC1

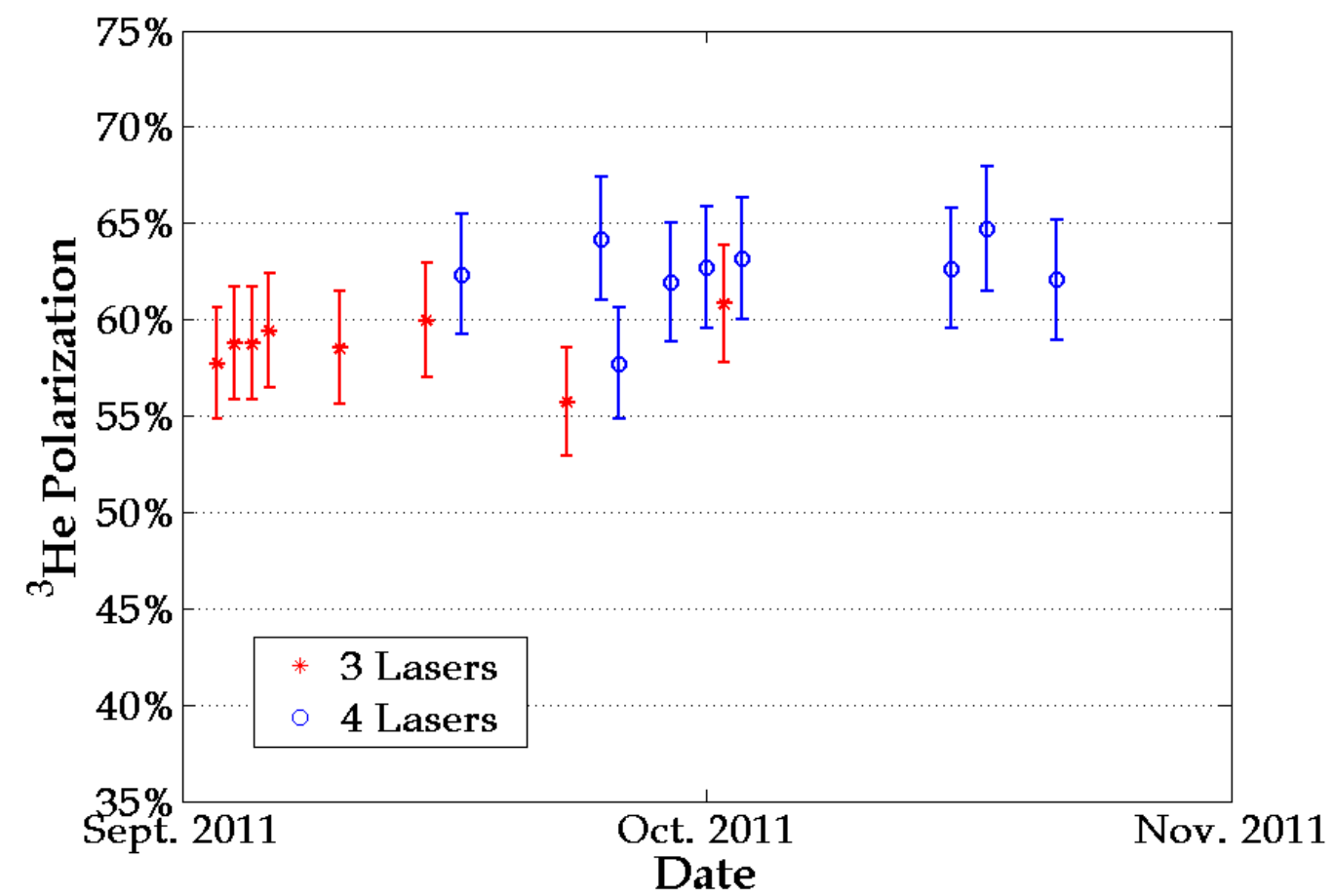

(b) HC2

Figure 3.17: Hybrid polarizer performance over time. Data points on these plots were taken from overnight spinups, so the durations range from 11-24 hours. The blue points were taken at the standard pressure $(P=120$ psig $)$ using $3 / 4$ lasers (HC1/HC2). The red points have either a different pressure or different number of lasers. The cell set temperature for all points was $225-235^{\circ} \mathrm{C}$. 


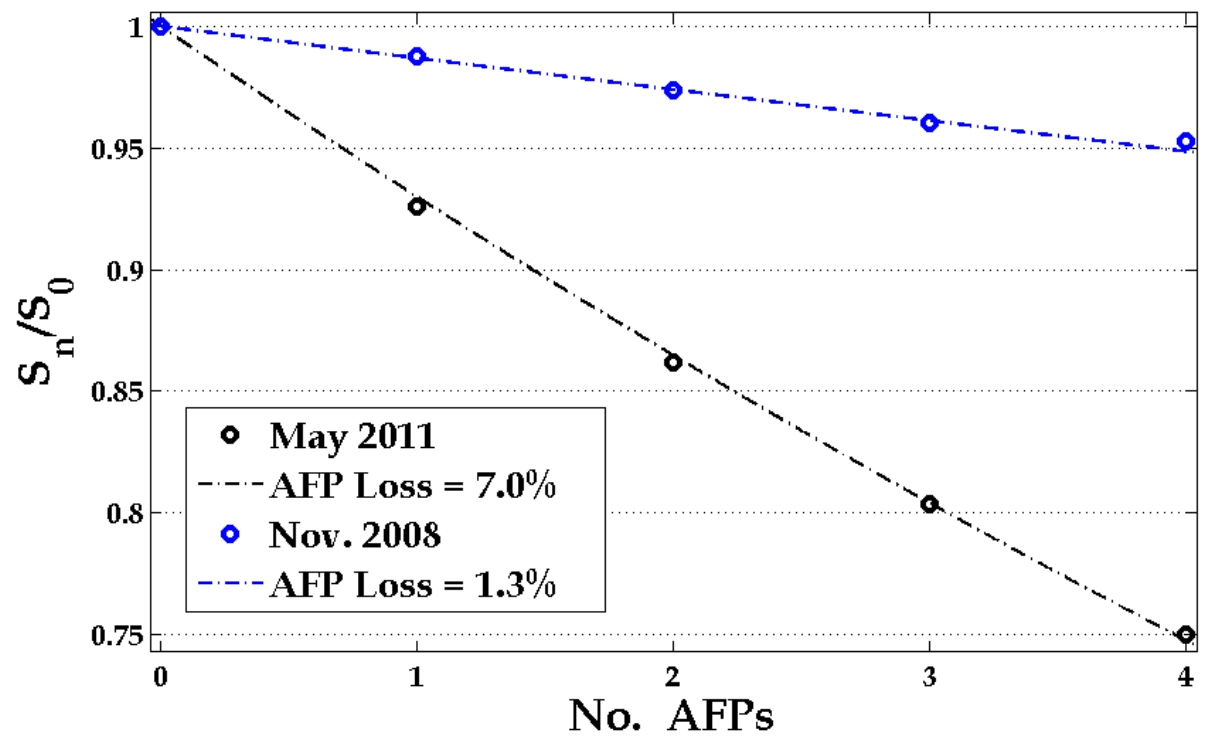

(a) AFP Loss per measurements from 2008 and 2011. Five AFP measurements are made at one minute intervals. The points are normalized to the first measurement and fit to a line which has a slope of percent loss per measurement.

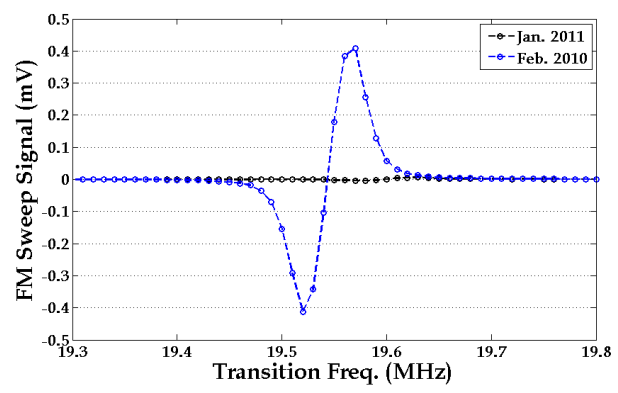

(b) EPR FM sweep signal amplitude measured in Feb 2010.

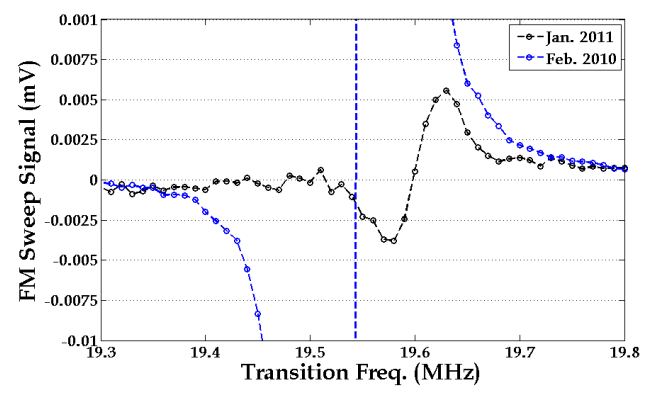

(c) Same graph as (b), zoomed in to show Jan 2011 data.

Figure 3.18: Hybrid polarizer diagnostic performance indicators. The AFP loss per measurement increased and the EPR FM sweep signal declined throughout the three-year lifetime of HC1. 


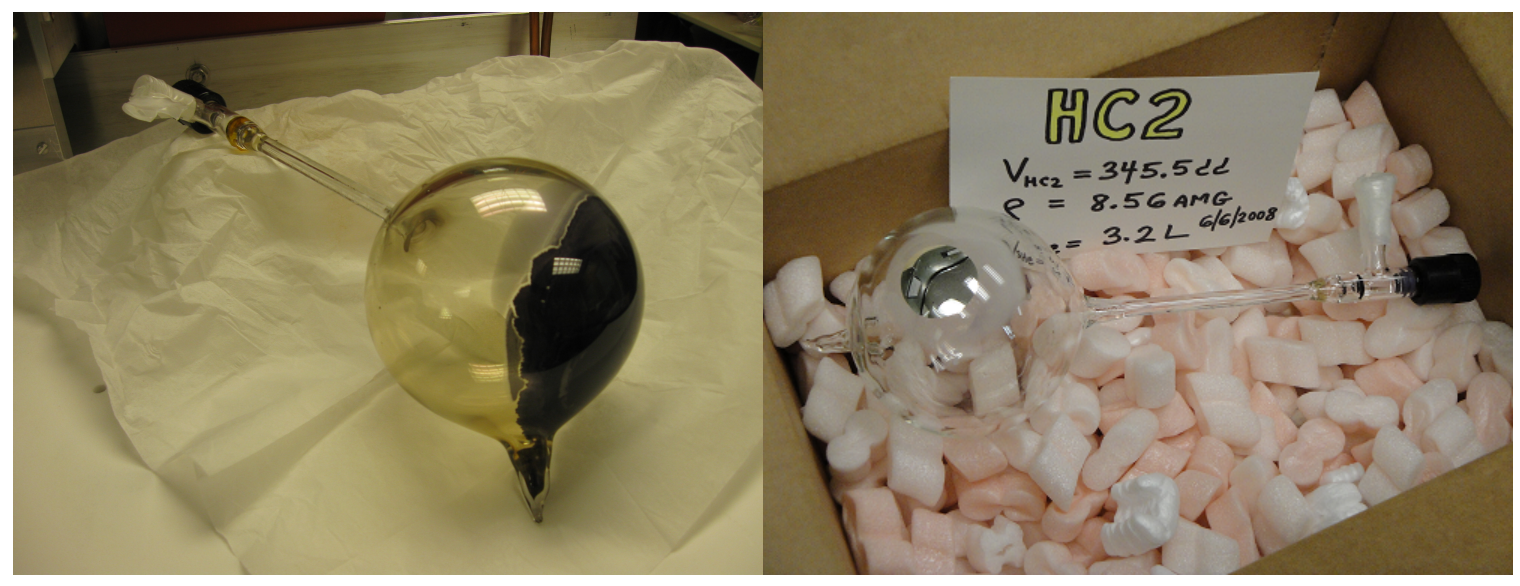

(a) Picture of HC1 after removal in June 2011.

(b) Picture of HC2 before installation.

Figure 3.19: Comparison of alkali metal pool in two polarizer cells. 


\subsection{Conclusions}

The Hybrid Polarizer represents a significant advance in performance over previous helium-3 polarizers used in medical imaging. Its peak performance was $\sim 65 \%$ helium-3 polarization overnight for $3.0 \mathrm{~L}$ of gas. The main features that drive this improvement are the use of narrowed lasers, hybrid alkali SEOP, and a well-optimized system. The larger capacity of the Hybrid Polarizer allows for the possibility of more than one imaging session per day if all of the gas is not used in the first session, as the polarization in the afternoon does not drop far from its peak value. The fast spinup time associated with hybrid SEOP means that the polarization cell could also be refilled and pumped to $\sim 40 \%$ helium-3 polarization in a few hours for a second same-day study if all of the gas were used in the first session.

The work of optimizing the technology used of the Hybrid Polarizer was initially done for nuclear target experiments, and the field of helium-3 lung imaging has been able to share in the benefits. The Hybrid Polarizer enables more efficient use of helium-3 in imaging as the cost of the gas is on the rise. Two of the key features on the Hybrid Polarizer, the narrowed lasers and the hybrid alkali mixture, could be added to existing helium-3 SEOP polarizers with relatively little effort and should produce significant polarization gains across the helium-3 lung imaging community. 


\section{Chapter 4}

\section{Steady State Free Precession}

The term steady state free precession (SSFP) describes a class of pulse sequences which are coherent, which means the magnetization vector acted on by each RF excitation has both a longitudinal and transverse component [64]. The repetition time (TR) between excitation RF pulses is shorter than the transverse magnetization decay constant $\left(T R<T_{2}\right.$ ) and the signal measured in the transverse plane consists of a mixture of the longitudinal and transverse magnetizations from the previous excitation. In contrast, incoherent pulse sequences drive the transverse magnetization to zero before the next excitation either by waiting long enough for it to decay $\left(T R>>T_{2}\right)$, by actively destroying the magnetization coherence by applying a dephasing gradient pulse (gradient spoiling), or by constantly changing the axis of each RF excitation pulse (RF spoiling). Therefore, the signal measured by an incoherent pulse sequence depends only on the longitudinal magnetization immediately before the RF excitation.

Coherent pulse sequences generally yield higher signal than incoherent pulse sequences due to the transverse magnetization being "recycled" by the RF excitation into the next TR period. The SSFP pulse sequence produces the highest signal per unit time in conventional MR of thermal-equilibrium magnetization [65]. 
SSFP pulse sequences have also been used for hyperpolarized gas MR ventilation imaging, and can yield a three-fold increase in signal-to-noise ratio over incoherent pulse sequences [66].

This thesis presents the first use of SSFP pulse sequences to generate diffusionweighted images of hyperpolarized gases. For reasons that will be discussed in Section 4.2 , it is highly advantageous to use the minimum repetition time possible when imaging hyperpolarized gases with an SSFP pulse sequence ( $T R \leq 4 \mathrm{~ms}$ ). Such short values of TR make it difficult or impossible to accommodate the bipolar diffusion gradients commonly used in previous hyperpolarized gas diffusion MR, which have diffusion times of $\Delta=1-2 \mathrm{~ms}$ (Chapter 2), in the same TR period as imaging gradients. In order to incorporate diffusion-weighting into an SSFP pulse sequence, one is therefore forced use shorter diffusion times. Since we are motivated to measure ADC values at shorter diffusion times, due to the potential for enhanced sensitivity to disease-related alterations of lung microstructure, and the high signal levels generated by SSFP pulse sequences will enhance our ability to measure the small signal attenuation that can be generated by the diffusion gradients achievable at short diffusion times, SSFP pulse sequences are an attractive option for measuring short time-scale diffusion.

This chapter describes the behavior of hyperpolarized magnetization under the influence of an SSFP pulse sequence, and the use of this behavior to develop methods for incorporating diffusion-weighting into the SSFP pulse sequence. First, I provide a description of RF excitation as it is implemented in an SSFP pulse sequence. Next, I discuss the equations used to model the effect of an SSFP pulse sequence (shown in Figure 4.2) on thermal equilibrium magnetization. In Section 4.3, I use the same formalism to derive a closed-form expression for the steady state behavior of hyperpolarized magnetization. We introduce diffusion sensitiza- 
tion through several different methods and derive the altered steady state magnetization behavior for each. Each closed-form expression was compared with magnetization simulations to verify the results and guide the pulse sequence design. The steady state equations were derived assuming on-resonant spins, but the effects of off-resonant magnetization were incorporated into the simulations and contributed greatly to determining how useful a particular method would be for obtaining quantitative measurements of hyperpolarized-gas diffusion in the lung. The resulting diffusion-weighted SSFP pulse sequences represent a balance of considerations such as minimal TR and data acquisition timing within the TR period.

\subsection{RF Excitation}

In Chapter 2, I discussed how a perpendicular resonant field will induce rotation of a spin out of alignment with the main magnetic field into the transverse plane. The relative phase between the applied RF pulse and the rotation of the particle determines the direction of that rotation, as illustrated in Figure 4.1. The transverse magnetization $M_{T}$ is not precessing in the rotating frame of $\hat{x}^{\prime}-\hat{y}^{\prime}$. Our convention is that an RF excitation of flip angle $\theta$ with zero phase produces counter-clockwise rotation about the $\hat{x}^{\prime}$-axis, and an RF pulse with a phase of $180^{\circ}(-\theta)$ produces clockwise rotation about the $\hat{x}^{\prime}$-axis.

In the SSFP pulse sequences we explored, the magnitude of the flip angle is the same for each RF pulse, but the phase alternates by $180^{\circ}$ between successive excitations. The switching can be thought of in two ways: either the sign of the angle rotation is constant, but the axis about which the spins rotate changes $180^{\circ}$, or the axis of rotation is constant but the sign of the rotation angle alternates (switching between $\pm \theta$ ). The result of an RF excitation with alternating phase is to produce transverse magnetization with alternating phase as well (Figure 4.1). 


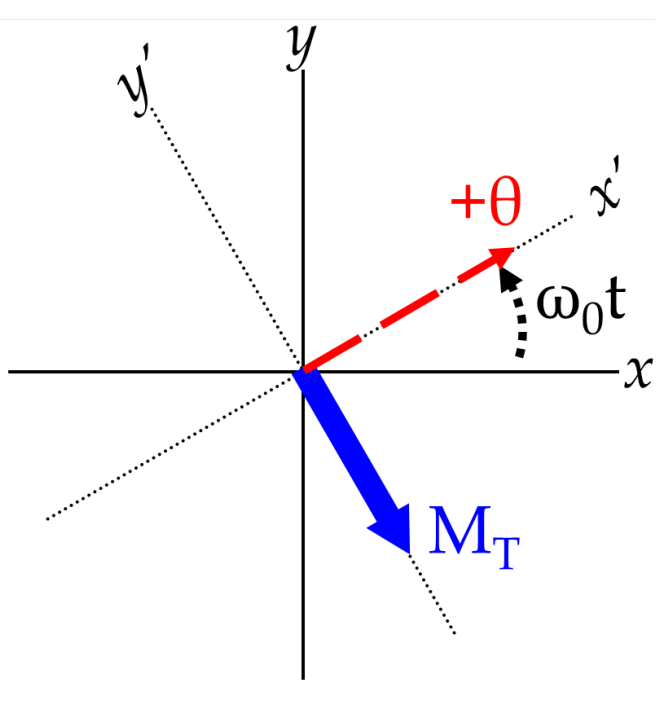

(a) $+\theta$

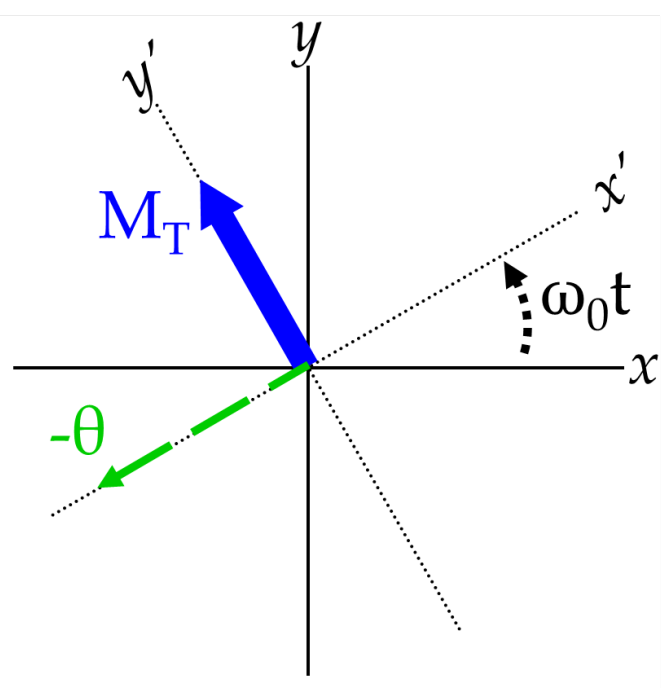

(b) $-\theta$

Figure 4.1: The rotating frame $\hat{x}^{\prime}-\hat{y}^{\prime}$ is determined by the resonant frequency of the spins in the holding field, $\omega_{0}=\gamma B_{0}$. The transverse magnetization $M_{T}$ is rotated counter-clockwise about the $\hat{x}^{\prime}$-axis by a positive flip angle (a), and clockwise by a negative flip angle (b).

\subsection{Magnetization Evolution under Steady State Free Precession}

When an SSFP pulse sequence like the one shown in Figure 4.2 is applied to thermal equilibrium polarization, the magnetization evolution eventually reaches a steady state in which the longitudinal and transverse components of the magnetization following each RF excitation are constant. Due to the alternating phase of the RF excitations, the transverse signal will experience some refocusing of offresonant phase accumulation, similar to the effect in spin echo pulse sequences discussed in Chapter 2. The refocusing due to RF phase alternation occurs even for the low flip angles used with hyperpolarized magnetization. Figure 4.3 demonstrates the alternating behavior of the signal, with $T_{1}$ and $T_{2}$ relaxation suppressed. Throughout the time between RF pulses, the transverse and longitudinal compo- 

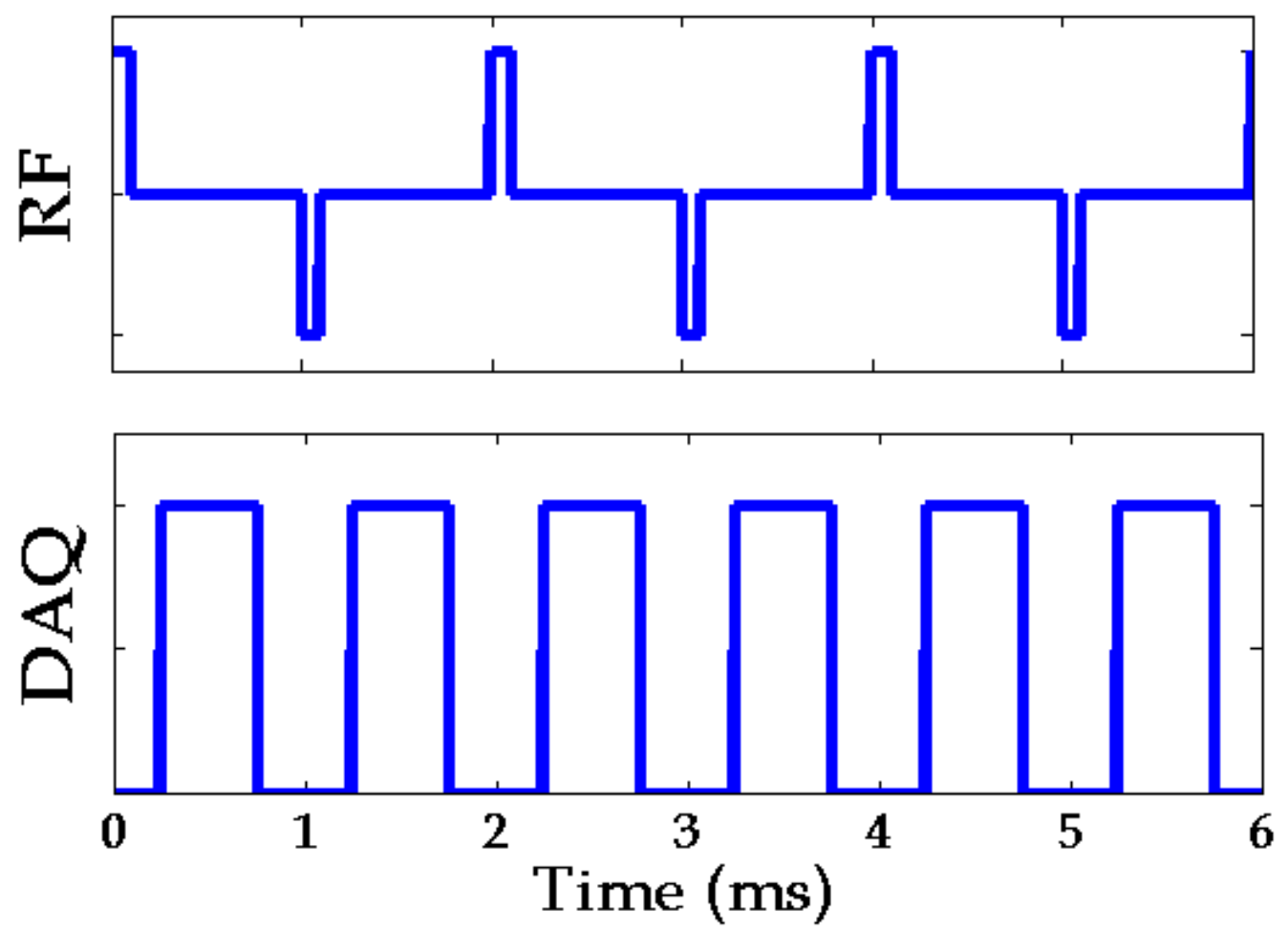

Figure 4.2: A non-imaging version of a steady state free precession pulse sequence with excitation RF pulses that alternate between flip angles of $\pm \theta$.

nents are relaxing toward thermal equilibrium. However, the signal is the same after each RF excitation at any point in the TR period.

The magnetization evolution under the influence of an SSFP pulse sequence is modeled as follows: we begin by assuming a purely longitudinal initial magnetization,

$$
\mathbf{M}(0)=\left(\begin{array}{c}
0 \\
0 \\
M_{i}
\end{array}\right),
$$

where the components of the vector $\mathbf{M}$ are the magnitudes of the magnetization in the $\hat{x}^{\prime}, \hat{y}^{\prime}$, and $\hat{z}$ directions. In the case of thermal equilibrium polarization, $M_{i} \equiv$ $M_{0}$ the thermal equilibrium value of magnetization. To take full advantage of the refocusing effects of alternating the excitation RF phase, the signal is measured at 


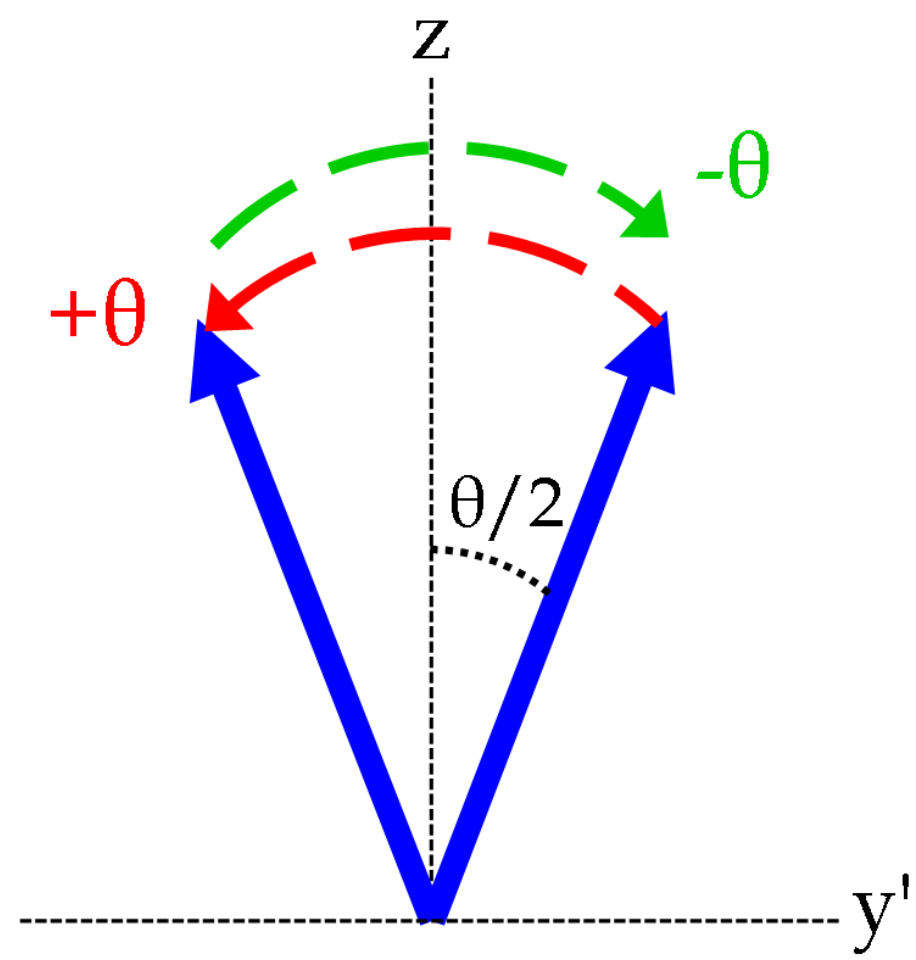

Figure 4.3: In steady state, the magnetization vector alternates orientation between $\pm \theta / 2$. The relaxation behavior has been suppressed in this picture.

$t=\mathrm{TR} / 2$. The measured signal evolves from one TR period to the next via the following matrix equation:

$$
\mathbf{M}(n+1)=\Gamma_{\text {half }} * R_{ \pm}(\theta) * \Gamma_{\text {half }} * \mathbf{M}(n)
$$

where $\mathbf{M}(n)$ is the magnetization vector after the n-th RF excitation pulse, $\Gamma_{\text {half }}$ is a relaxation matrix for evolving the magnetization through half of a TR period, and $R_{ \pm}$is the rotation matrix associated with each RF pulse, which alternates between $\pm \theta$. The RF excitations are assumed to rotate the magnetization about the $\hat{x}^{\prime}$-axis, 
for which the rotation matrix is:

$$
R_{ \pm}( \pm \theta)=\left(\begin{array}{ccc}
1 & 0 & 0 \\
0 & \cos \theta & \pm \sin \theta \\
0 & \mp \sin \theta & \cos \theta
\end{array}\right)
$$

A positive flip angle $+\theta$ corresponds to a counter-clockwise rotation about $\hat{x}^{\prime}$. The relaxation matrix is:

$$
\Gamma_{\text {half }}=\left(\begin{array}{ccc}
e^{-\frac{T R / 2}{T 2}} & 0 & 0 \\
0 & e^{-\frac{T R / 2}{T 2}} & 0 \\
0 & 0 & e^{-\frac{T R / 2}{T 1}}+M_{0}\left(1-e^{-\frac{T R / 2}{T 1}}\right)
\end{array}\right)
$$

In hyperpolarized magnetization, the thermal equilibrium polarization is negligible compared to the hyperpolarized longitudinal magnetization $\left(M_{0} \approx 0\right)$ and $\Gamma_{\text {half }}$ becomes:

$$
\Gamma_{\text {half }}=\left(\begin{array}{ccc}
e^{-\frac{T R / 2}{T 2}} & 0 & 0 \\
0 & e^{-\frac{T R / 2}{T 2}} & 0 \\
0 & 0 & e^{-\frac{T R / 2}{T 1}}
\end{array}\right)
$$

The simulations of magnetization evolution that will be described in this chapter are based on iteratively applying Equation 4.2 to a magnetization vector using the matrices defined in Equation 4.3 and either Equation 4.4 or 4.5. Each simulation began with the initial magnetization vector shown in equation 4.1 , with $M_{i}=1$. The $\hat{x}^{\prime}, \hat{y}^{\prime}$, and $\hat{z}$ components of the magnetization were calculated at three time points in each TR period, which are denoted by the vectors $\mathbf{M}_{+}(n)$ (immediately following the n-th RF excitation), $\mathbf{M}(n)$ (at $t=T R / 2)$, and $\mathbf{M}_{-}(n+1)$ (immediately before the next RF pulse).

We first simulated the evolution of the magnetization vector for thermal equi- 
librium polarization. The input parameters to the simulation were chosen to be physically realistic: $T R=5 \mathrm{~ms}, \theta=5^{\circ}, T_{1}=2 \mathrm{~s}$ and $T_{2}=100 \mathrm{~ms}$. The simulation ran for 2000 RF excitations. At the end of this simulation, the evolutions of the $\mathbf{M}_{+}$(1999) and $\mathbf{M}_{+}$(2000) magnetization vectors were then evolved over their respective TR periods using the relaxation matrix:

$$
\mathbf{M}(t)=\left(\begin{array}{ccc}
e^{-\frac{t}{T 2}} & 0 & 0 \\
0 & e^{-\frac{t}{T 2}} & 0 \\
0 & 0 & e^{-\frac{t}{T 1}}+M_{0}\left(1-e^{-\frac{t}{T 1}}\right)
\end{array}\right) * \mathbf{M}_{+}(2000) .
$$

The results of this calculation are shown in Figure 4.4. The magnitude of the transverse magnetization, $M_{y^{\prime}}(t)$ is plotted versus the magnitude of the longitudinal magnetization, $M_{z}(t)$, over the course of consecutive TR periods. The signal evolution begins at position 1, immediately following an RF pulse of flip angle $+\theta$. From position 1 to 2 relaxation occurs, resulting in an increased longitudinal component and a decreased transverse component. The RF excitation of $-\theta$ rotates the vector from position 2 to 3 . Relaxation from position 3 to position 4 again increases the longitudinal component and decreases the transverse component.

This simulation allows us to understand the qualitative features of the steadystate magnetization for representative physical parameters. We see that the magnetization reaches a steady state in which:

$$
\mathbf{M}(n)=\mathbf{M}(n+1) .
$$

The equilibrium magnetization $\mathbf{M}(\infty)$ must satisfy Equation 4.2:

$$
\mathbf{M}(\infty)=\Gamma_{\text {half }} * R_{ \pm}(\theta) * \Gamma_{\text {half }} * \mathbf{M}(\infty)
$$




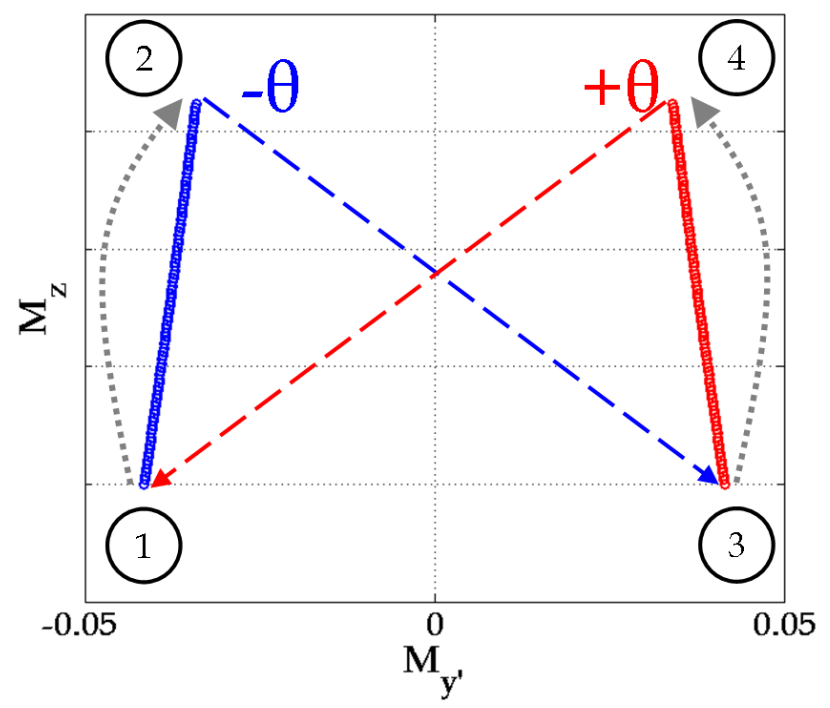

Figure 4.4: Simulated magnetization magnitude at steady state (after 2000 RF excitations) during two consecutive TR windows for thermal equilibrium polarization. Magnetization evolves from 1 to 2 and 3 to 4 via $T_{1}$ and $T_{2}$ relaxation (dotted gray lines). Rotation due to RF excitations (dashed lines) move the magnetization vector from 2 to 3 and 4 to 1 . Simulation parameters were: $T R=5 \mathrm{~ms}, T_{1}=2 \mathrm{~s}$, $\mathrm{T}_{2}=100 \mathrm{~ms}$, and $\theta=5^{\circ}$.

Using this equality and Equations 4.3 and 4.4, the steady state transverse magnetization $\left(M_{y^{\prime}}(\infty)\right)$ can be calculated for the general case:

$$
M_{y^{\prime}}(\infty)=M_{0} e^{-\frac{T R}{T_{2}}}\left(1-e^{-\frac{T R}{T_{1}}}\right) \frac{\sin \theta\left(1-e^{-\frac{T R}{T_{2}}}\right)}{x}
$$

where:

$$
x \equiv\left(1-e^{-\frac{T R}{T_{1}}} \cos \theta\right)\left(1-e^{-\frac{T R}{T_{2}}}\right)-e^{-\frac{T R}{T_{2}}}\left(e^{-\frac{T R}{T_{1}}}-\cos \theta\right)\left(e^{-\frac{T R}{T_{2}}}-1\right) .
$$

Equation 4.9 is well-studied, and used in conventional MR to optimize the choice of SSFP pulse sequence parameters. We use the same formalism to guide a derivation of the steady state signal for hyperpolarized magnetization in the next section. 


\subsection{Steady State Free Precession in Hyperpolarized Gas}

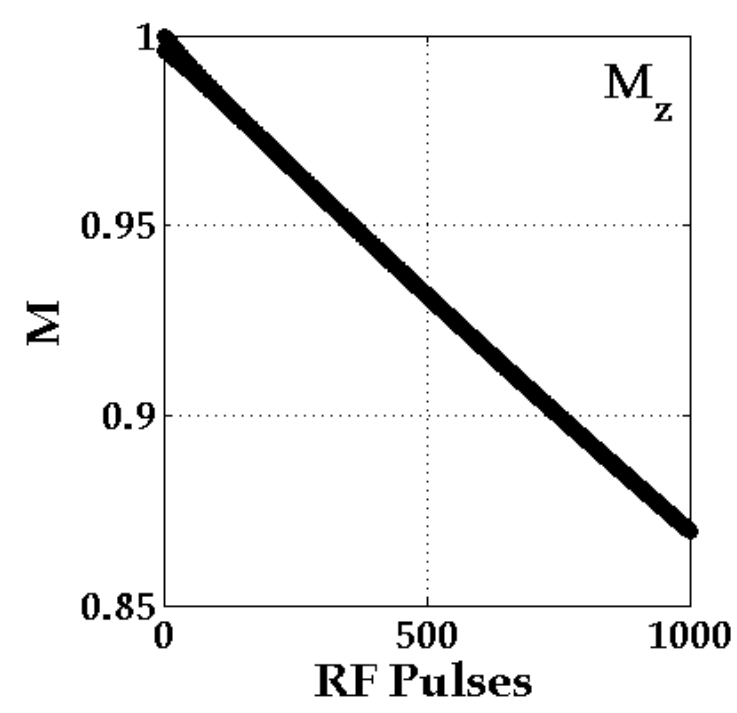

(a) Longitudinal Magnetization

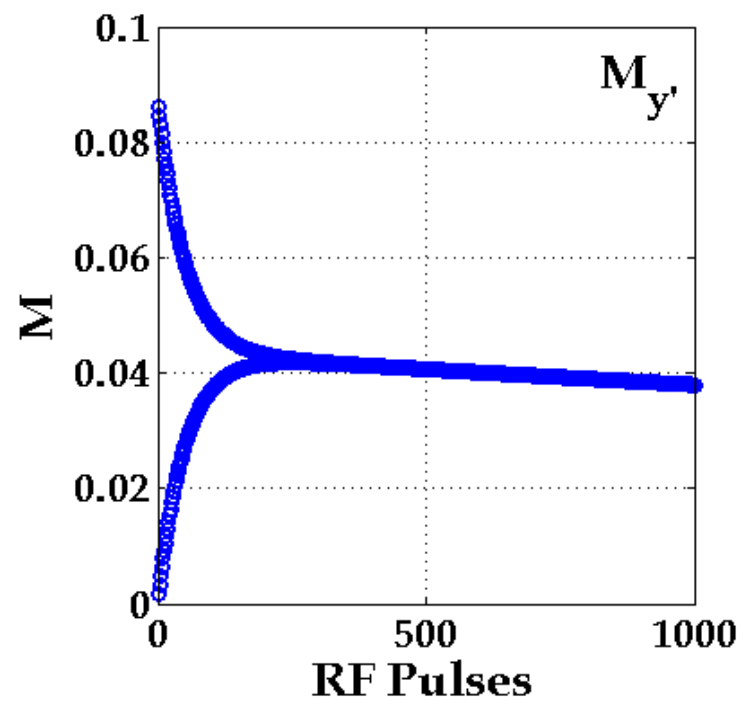

(b) Transverse Magnetization

Figure 4.5: Simulated (a) longitudinal and (b) transverse magnetization for hyperpolarized media and an SSFP pulse sequence with $T R=2 m s, T_{1}=20 s, T_{2}=$ $100 \mathrm{~ms}$, and $\theta=5^{\circ}$.

The difference between thermal equilibrium magnetization and hyperpolarized magnetization is that the longitudinal component decays instead of regrowing following each RF excitation, as reflected in Equation 4.5. We next simulated the evolution of hyperpolarized magnetization using the formalism developed in the previous section. Once again, the input parameters were chosen to be physically realistic: $T R=5 \mathrm{~ms}, T_{1}=20 \mathrm{~s}, T_{2}=100 \mathrm{~ms}$, and $\theta=5^{\circ}$ and the simulation ran for 2000 RF excitations. At the end of the simulation the evolutions of the $\mathbf{M}_{+}$(1998), $\mathbf{M}_{+}$(1999), and $\mathbf{M}_{+}$(2000) magnetization vectors were then calculated over their respective TR periods using the relaxation matrix in Equation 4.6 but with $M_{0}=0$. The simulated magnetization is shown in Figure 4.5 versus RF excitation $n$. The longitudinal component after each RF excitation (Figure 4.5a) has an exponential decline due to both $T_{1}$ and the excitation RF pulses. The transverse magnetization 
in Figure 4.5b initially oscillates in the approach to steady state before reaching a constant rate of decay.

Figure 4.6 illustrates the magnetization behavior during the time between RF pulses once the system has reached steady state, over the course of three consecutive TR periods for hyperpolarized magnetization. For the purposes of demonstration, $T_{2}$ was shortened to $50 \mathrm{~ms}$ to emphasize the transverse signal decay. The signal evolution begins at position 1, immediately following an RF pulse of flip angle $+\theta$. From position 1 to 2 relaxation occurs, resulting in a decreased longitudinal and transverse magnetization magnitudes. The RF excitation of $-\theta$ rotates the vector from position 2 to 3 . Relaxation from position 3 to position 4 again decreases both the longitudinal and transverse magnitudes. The RF excitation of $+\theta$ rotates the vector from position 4 to 5 . Another period of relaxation from position 5 to 6 reduces both components.

Figure 4.7 plots the steady state transverse magnetization ratio $M_{y^{\prime}}(n+1) / M_{y^{\prime}}(n)$ from the SSFP magnetization simulation in Figure 4.5b. This plot shows that the ratio of consecutive transverse magnetizations is constant, even as the absolute signal levels decline.

As in the thermally-polarized case, our simulation allows us to understand the qualitative features of the magnetization evolution in the hyperpolarized case. Now we can use Equations 4.2, 4.3, and 4.5 to derive a closed-form expression for the steady state signal ratio $\mathbf{M}(n+1) / \mathbf{M}(n)$ produced by an SSFP pulse sequence acting on hyperpolarized magnetization. We use the steady state nature of the system,

$$
\frac{\mathbf{M}(n+1)}{\mathbf{M}(n)}=\frac{\mathbf{M}(n+2)}{\mathbf{M}(n+1)}
$$

to derive an expression for the ratio strictly in terms of TR, $T_{1}, T_{2}$, and $\theta$. We use the following shorthand notation for expressions which appear in the relaxation 


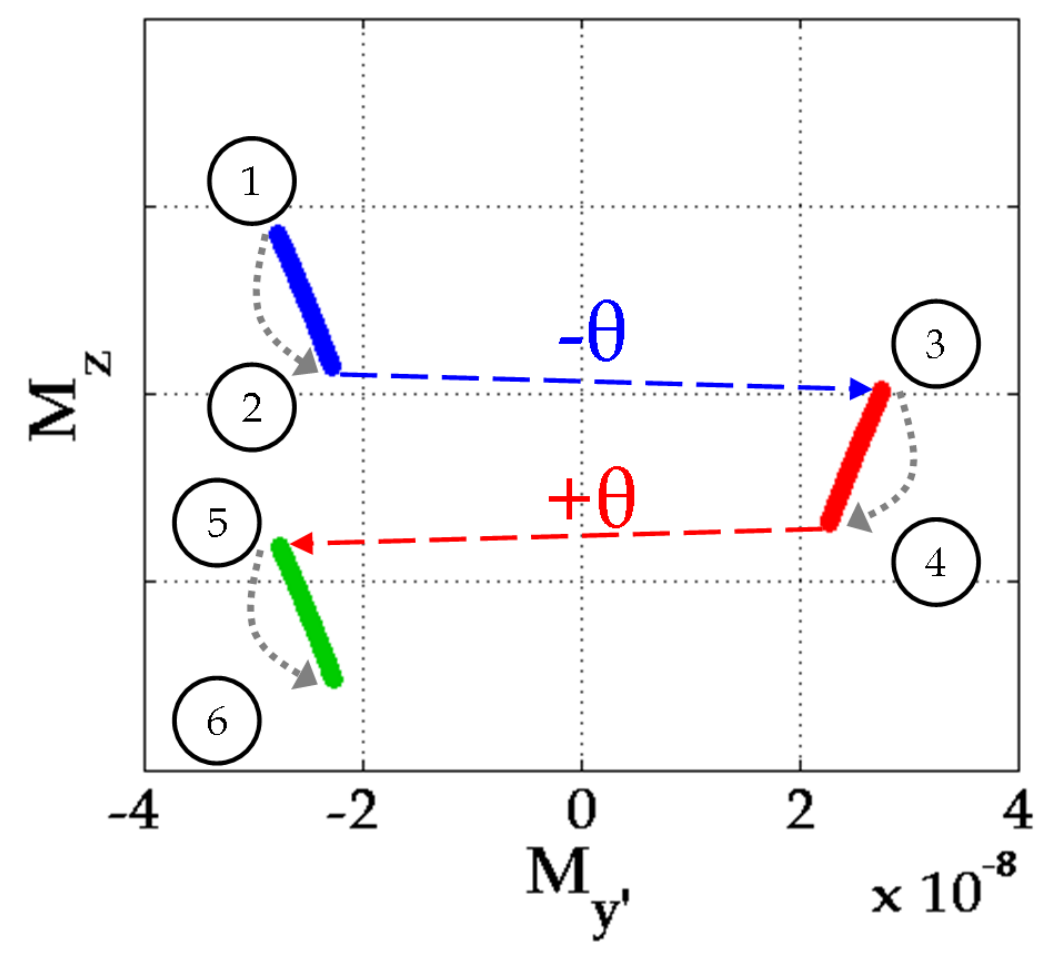

Figure 4.6: Simulated magnetization magnitude at steady state (after 2000 RF excitations) during three consecutive TR windows for hyperpolarized magnetization. Simulation parameters were $T R=5 \mathrm{~ms}, T_{1}=20 \mathrm{~s}, T_{2}=50 \mathrm{~ms}$, and $\theta=5^{\circ}$. The dashed lines represent the rotation effects of an RF excitation pulse and the dotted gray lines represent relaxation effects from $T_{1}$ and $T_{2}$ during the TR period.

and rotation matrices:

$$
\begin{gathered}
E_{1} \equiv e^{-\frac{T R}{T 1}}, \quad E_{2} \equiv e^{-\frac{T R}{T 2}}, \quad s \equiv \sin \theta, \quad c \equiv \cos \theta \\
\sqrt{E_{1}} \equiv e^{-\frac{T R / 2}{T 1}}, \quad \sqrt{E_{2}} \equiv e^{-\frac{T R / 2}{T 2}}
\end{gathered}
$$

. Here we assume that the magnetization is already at steady state, and thus has 


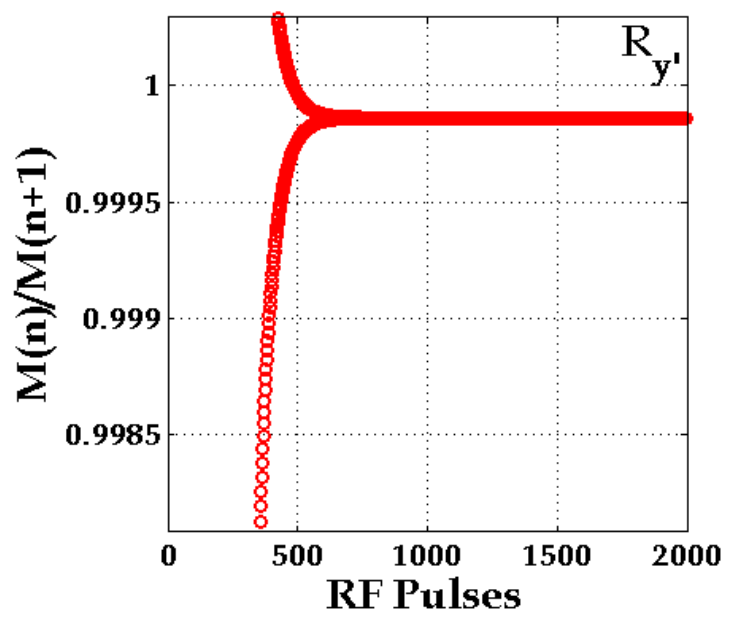

Figure 4.7: Simulated transverse signal ratio $M_{y^{\prime}}(n+1) / M_{y^{\prime}}(n)$ for hyperpolarized media and an SSFP pulse sequence with TR $=2 m s, T_{1}=20 s, T_{2}=$ $100 \mathrm{~ms}$, and $\theta=5^{\circ}$.

both transverse and longitudinal components:

$$
\mathbf{M}(n)=\left(\begin{array}{c}
0 \\
M_{y^{\prime}} \\
M_{z}
\end{array}\right)
$$

The magnetization is measured at $t=T R / 2$, and evolves between measurements as described previously:

$$
\mathbf{M}(n+1)=\Gamma_{\text {half }} * R_{-}(\theta) * \Gamma_{\text {half }} * \mathbf{M}(n),
$$

where we have arbitrarily chosen to start with $R_{-}(\theta)$. We apply the relaxation and rotation matrices to $\mathbf{M}(n)$ in order to obtain $\mathbf{M}(n+1)$ :

$$
\mathbf{M}(n+1)=\left(\begin{array}{c}
0 \\
c E_{2} M_{y^{\prime}}(n)-s \sqrt{E_{1} E_{2}} M_{z}(n) \\
s \sqrt{E_{1} E_{2}} M_{y^{\prime}}(n)+c E_{1} M_{z}(n)
\end{array}\right) .
$$


Next we apply Equation 4.2 with $R_{+}(\theta)$ to $M(n+1)$ to get $M(n+2)$ :

$$
\begin{aligned}
& \mathbf{M}(n+2)=\Gamma_{\text {half }} * R_{+}(\theta) * \Gamma_{\text {half }} * \mathbf{M}(n+1) \\
& =\left(\begin{array}{c}
0 \\
c E_{2} M_{y^{\prime}}(n+1)+s \sqrt{E_{1} E_{2}} M_{z}(n+1) \\
-s \sqrt{E_{1} E_{2}} M_{y^{\prime}}(n+1)+c E_{1} M_{z}(n+1)
\end{array}\right) .
\end{aligned}
$$

Substituting the components of $\mathbf{M}(\mathbf{n}+\mathbf{1})$ from Equation 4.15:

$$
\mathbf{M}(n+2)=\left(\begin{array}{c}
0 \\
\left(c^{2} E_{2}^{2}+s^{2} E_{1} E_{2}\right) M_{y^{\prime}}(n)+c s \sqrt{E_{1} E_{2}}\left(E_{1}-E_{2}\right) M_{z}(n) \\
c s \sqrt{E_{1} E_{2}}\left(E_{1}-E_{2}\right) M_{y^{\prime}}(n)+\left(s^{2} E_{1} E_{2}+c^{2} E_{1}^{2}\right) M_{z}(n)
\end{array}\right)
$$

We focus on the transverse $\left(\hat{y}^{\prime}\right)$ component, as it is proportional to the signal measured by the MR scanner. The left side of Equation 4.11 becomes:

$$
\frac{M_{y^{\prime}}(n+1)}{M_{y^{\prime}}(n)}=c E_{2}-s \sqrt{E_{1} E_{2}} \frac{M_{z}(n)}{M_{y^{\prime}}(n)} .
$$

We can rearrange Equation 4.11 as follows:

$$
M_{y^{\prime}}(n+1) * M_{y^{\prime}}(n+1)=M_{y^{\prime}}(n+2) * M_{y^{\prime}}(n),
$$

and use the expression for $M_{y^{\prime}}(n+2)$ to solve for the quantity $\frac{M_{z}(n)}{M_{y^{\prime}}(n)}$. Substituting in the expressions for $M_{y^{\prime}}(n+1)$ and $M_{y^{\prime}}(n+2)$ into Equation 4.20 and simplifying, we have: 


$$
\begin{array}{r}
-c s E_{2} \sqrt{E_{1} E_{2}} M_{y^{\prime}} M_{z}+s^{2} E_{1} E_{2} M_{z}^{2} \\
=\left(s^{2} E_{1} E_{2}\right) M_{y^{\prime}}^{2}+c s \sqrt{E_{1} E_{2}}\left(E_{1}\right) M_{z} M_{y^{\prime}} .
\end{array}
$$

Now we rearrange terms and divide by $M_{y^{\prime}}^{2}$ to get an expression involving $M_{z} / M_{y^{\prime}}$ :

$$
s^{2} E_{1} E_{2}\left(\frac{M_{z}}{M_{y^{\prime}}}\right)^{2}-c s \sqrt{E_{1} E_{2}}\left(E_{2}+E_{1}\right) \frac{M_{z}}{M_{y^{\prime}}}-s^{2} E_{1} E_{2}=0 .
$$

Solving this quadratic equation for $M_{z} / M_{y^{\prime}}$ :

$$
\begin{gathered}
\frac{M_{z}}{M_{y^{\prime}}}=\frac{c s \sqrt{E_{1} E_{2}}\left(E_{1}+E_{2}\right) \pm \sqrt{c^{2} s^{2} E_{1} E_{2}\left(E_{2}+E_{1}\right)^{2}+4 s^{4} E_{1}^{2} E_{2}^{2}}}{2 s^{2} E_{1} E_{2}} \\
=\frac{c\left(E_{1}+E_{2}\right) \pm \sqrt{c^{2}\left(E_{2}^{2}+E_{1}^{2}\right)+2 E_{1} E_{2}\left(1+s^{2}\right)}}{2 s \sqrt{E_{1} E_{2}}} .
\end{gathered}
$$

Finally, we substitute Equation 4.24 into Equation 4.19 to get a closed-form expression for the ratio $M_{y^{\prime}}(n+1) / M_{y^{\prime}}(n)$ in terms of $T_{1}, T_{2}, \mathrm{TR}$, and $\theta$ :

$$
\frac{M_{y^{\prime}}(n+1)}{M_{y^{\prime}}(n)}=-\frac{c}{2}\left(E_{1}-E_{2}\right) \pm \frac{\sqrt{c^{2}\left(E_{2}^{2}+E_{1}^{2}\right)+2 E_{1} E_{2}\left(1+s^{2}\right)}}{2}
$$

Equation 4.25 defines the relationship between any two consecutive signal magnitudes assuming on-resonance magnetization. The ratio is a negative number since the transverse components alternate sign at each RF excitation. The expression has a complicated dependence on both the pulse sequence parameters $T R$ and $\theta$ and the relaxation parameters $T_{1}$ and $T_{2}$. In Figure 4.8, we have plotted the absolute 
values of both the solutions $( \pm)$ and the magnetization simulation for parameters common in hyperpolarized helium-3 MR in humans: $T_{1} \approx 20 s, T_{2} \approx 100 \mathrm{~ms}$, and $\theta=5^{\circ}$.

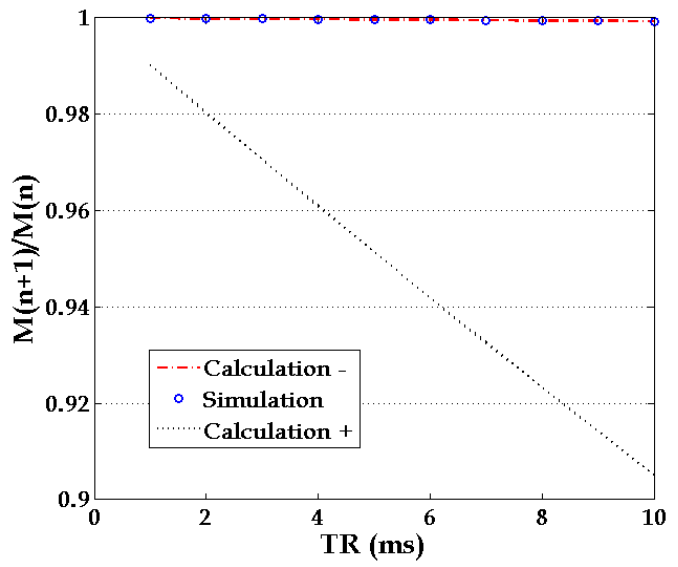

(a)

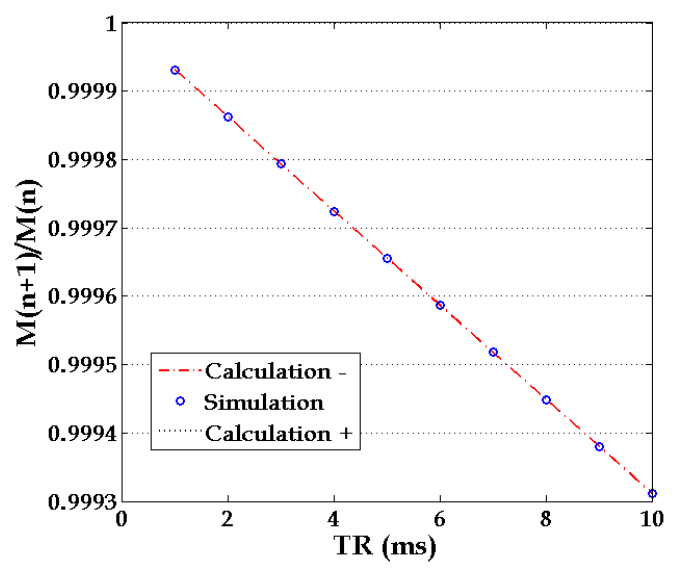

(b) (a) on smaller scale

Figure 4.8: Plot of simulation results (blue points) and Equation 4.25 minus/plus solutions (red dashed line/black dotted line) versus TR. (a) Shows all three plots, and (b) has been zoomed in to show the overlap of the magnetization simulation and Equation $4.25(-)$. Relaxation and excitation values used were $T_{1}=20 \mathrm{~s}, T_{2}=$ $100 \mathrm{~ms}, \theta=5^{\circ}$.

The (-) solution matches the output of our simulation, so we take this to be the correct physical description of the system:

$$
\frac{M_{y^{\prime}}(n+1)}{M_{y^{\prime}}(n)}=-\frac{c}{2}\left(E_{1}-E_{2}\right)-\frac{\sqrt{c^{2}\left(E_{2}^{2}+E_{1}^{2}\right)+2 E_{1} E_{2}\left(1+s^{2}\right)}}{2} .
$$

For the relaxation and excitation parameters simulated, the steady state ratio $M_{y^{\prime}}(n+$ 1) $/ M_{y^{\prime}}(n)=0.9993 \sim 0.9999$ is only slightly less than unity for the range of repetition times plotted in Figure 4.8. By contrast, in an incoherent pulse sequence the consecutive signal ratio equals $\cos \theta$, which here would be $M_{y^{\prime}}(n+1) / M_{y^{\prime}}(n)=$ 0.996 for $\theta=5^{\circ}$. The order of magnitude difference in signal loss between pulses of the same flip angle in SSFP versus incoherent pulse sequences means that SSFP 
pulse sequences can use larger flip angles to obtain the same rate of signal decline over the course of an acquisition. That is, it takes a larger flip angle to "use up" the available hyperpolarized magnetization by the end of the scan. Since larger flip angles generate larger transverse magnetization and thus higher signal, SSFP pulse sequences are capable of producing higher signal-to-noise ratio in the acquired data.

\subsection{Effects of Off-resonant Magnetization}

In addition to the potential SNR advantage of SSFP over incoherent pulse sequences, there are other tradeoffs which will factor into our design of diffusionweighted SSFP pulse sequences. A disadvantage of coherent pulse sequences such as SSFP is sensitivity of the signal size to off-resonant magnetization. Spins which precess at the frequency of the rotating frame $\hat{x}^{\prime}-\hat{y}^{\prime}\left(\omega_{0}\right)$ are said to be on-resonance, while spins with either a higher or lower precession frequency are known as offresonant spins. The rotating-frame frequency is input to the MR scanner, and it determines the excitation frequency of the RF pulse and the demodulation frequency of the data acquisition. Most importantly for our application, it also determines the phase of the RF excitation and thus the axis about which the flip angle is applied.

The transverse components of on-resonance spins are stationary in the rotating frame. Off-resonance spins rotate in this frame and thus accumulate phase during the TR period, mixing the $\hat{y}^{\prime}$ and $\hat{x}^{\prime}$ components of the transverse magnetization. During the TR period, an off-resonant spin rotates in the $\hat{x}^{\prime}-\hat{y}^{\prime}$ plane through an angle we will refer to as $\beta$. To demonstrate the effects of off-resonant magnetization on the steady state signal, we modified the our simulation to allow for a rotation of the transverse magnetization component by an angle $\beta$ during each TR period.

Figure 4.9a shows the simulated steady-state evolution of the transverse mag- 
netization for several different values of $\beta: 100^{\circ}$ (red and blue curves), $130^{\circ}$ (green and black curves), or $160^{\circ}$ (purple and orange curves). The magnetization evolved from the point labeled with a plus sign to the point labeled with a diamond for each curve, with the $t=T R / 2$ point labeled with a circle. The transverse magnetization now has both $\hat{y}^{\prime}$ and $\hat{x}^{\prime}$ components, in contrast to the simulations plotted in Figures 4.4 and 4.6. Figure $4.9 \mathrm{~b}$ shows the total transverse magnitude $M_{T}=$ $\sqrt{M_{y^{\prime}}^{2}+M_{x^{\prime}}^{2}}$ measured at $t=T R / 2$ for the full range of possible precession angles $\beta=-180^{\circ} \rightarrow+180^{\circ}$ at two different flip angles, $\theta=5^{\circ}$ (blue circles) and $\theta=10^{\circ}$ (red stars).

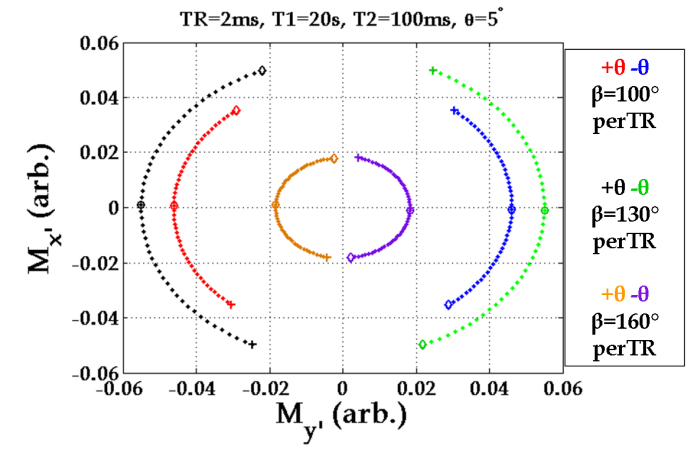

(a) Magnetization Evolution during TR.

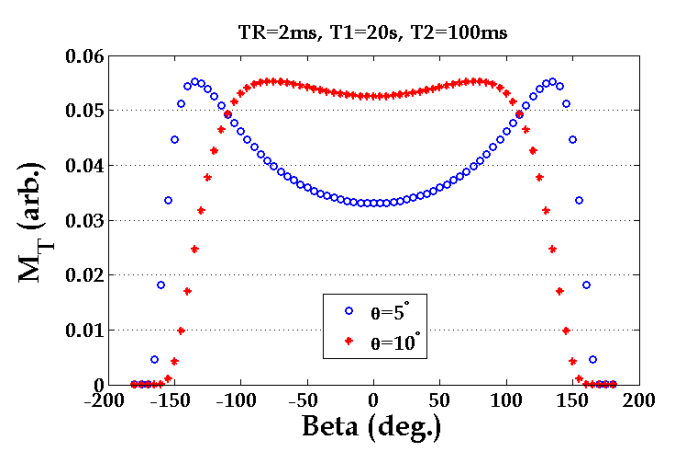

(b) Magnetization Vs $\beta$

Figure 4.9: (a) Plot of simulated transverse magnetization $\left(\hat{y}^{\prime}\right.$ vs. $\left.\hat{x}^{\prime}\right)$ for two consecutive TR periods in the presence of off-resonance: $\beta=\left[100^{\circ}, 130^{\circ}\right.$, and $160^{\circ}$ ] per TR. (b) The total transverse magnetization measured at $t=T R / 2$ from $\beta=[-180 \rightarrow+180]$ for $\theta=5^{\circ}, 10^{\circ}$. Simulation parameters for hyperpolarized magnetization were $\mathrm{TR}=2 \mathrm{~ms}, \mathrm{~T} 1=20 \mathrm{~s}$, and $\mathrm{T} 2=100 \mathrm{~ms}$.

For off-resonance precession angles of $\beta \leq 100^{\circ}$ per TR, the transverse magnetization magnitude measured at $t=T R / 2$ is nearly equal to the value in the on-resonance case $(\beta=0)$, as shown in Figure $4.9 \mathrm{~b}$. Thus at this flip angle, the measured signal will be relatively insensitive to off-resonant magnetization when $\beta \leq 100^{\circ}$ per TR. For larger values of $\beta$, the transverse magnetization is much lower than the on-resonance value at all times throughout the TR period. 
When using an SSFP pulse sequence to create an image, regions in which $\beta \sim$ $180^{\circ}$ per TR will not have appreciable signal, leading to dark areas in the final image. The main source of off-resonant magnetization precession is relatively smooth spatial variations in the main holding field of the MR scanner. Since the minimum signal occurs along contour lines where the resulting frequency offset corresponds to $\beta=180^{\circ}$, this effect usually appears in SSFP images as curved lines of greatly reduced signal, often referred to as "banding artifacts". The most common (and effective) means of suppressing off-resonance effects in an SSFP imaging pulse sequence is to reduce $T R$, which reduces $\beta$ proportionally. Another method of reducing signal loss due to off-resonant magnetization is to improve the active magnetic field shim to reduce macroscopic field variation across the sample, although this approach is impractical for hyperpolarized gas imaging. Since the off-resonance precession angle $\beta$ is also proportional to gamma, SSFP acquisitions are more susceptible to banding artifacts at higher holding fields.

While macroscopic field variations generally produce gentle variations in $\beta$ across the imaged volume, microscopic field inhomogeneities will result in a range of $\beta$ values that exist within a given sampling region. This type of environment is present in the lungs, where there are susceptibility-induced magnetic field gradients at the air/tissue interfaces. For a collection of $\beta$ values in the same sampling region, the measured signal is a coherent average over the magnitudes shown in Figure $4.9 \mathrm{~b}$. The effect of microscopic field variations is to reduce the net signal compared to the on-resonance value. When making diffusion measurements in the lung, both macroscopic and microscopic field variations are present. In developing an SSFP-based method for measuring diffusion in hyperpolarized gas MR, we therefore evaluated our pulse sequences under both of these conditions. 


\subsection{Preliminary SSFP Measurements}

Our calculations and simulations indicate that the ratio of consecutive signals at steady state should be $M(n+1) / M(n)=1$ for magnetization at thermal equilibrium, and $M(n+1) / M(n)<1$ for hyperpolarized magnetization. To confirm this theoretical result, we collected data using the basic global (non-imaging) SSFP sequence shown in Figure 4.2 in both a plastic water bottle phantom and a glass sphere phantom filled with hyperpolarized helium-3. A 1.5 T Siemens Magnetom Avanto MR scanner was used to acquire data (Siemens Medical Solutions, Malvern PA). The pulse sequence used for these tests had the following parameters: $T R_{H 1}=2.0 \mathrm{~ms}, T R_{H e 3}=2.1 \mathrm{~ms}, \theta_{H 1}=2^{\circ}, \theta_{H e 3}=4^{\circ}, \mathrm{RF}$ pulse duration $=$ $100 \mu$ s, and RF phase $=0^{\circ} / 180^{\circ}$. Figure 4.10 shows the signal measured at $t=$ $T R / 2$ using the SSFP pulse sequence in (a) the water sample and (b) the hyperpolarized helium-3 sample. Figure 4.10c graphs the ratio of consecutive points $M(n+1) / M(n)$ from the helium-3 data.

The raw data from these tests show a systematic difference between signal measurements acquired after RF pulses with flip angles of $+\theta$ and $-\theta$. In Figure 4.10a and (b), the blue points represent data taken after an excitation RF pulse with the phase set to $0^{\circ}(+\theta)$, and the red points represent RF phase set to $180^{\circ}(-\theta)$. The size of the difference between red and blue points is similar in water and helium, $\sim 4 \%$ of the total signal level.

While such a "baseline difference" has not been reported previously in a global acquisition, it seems to be present in data taken by Wild, et al in the form of the small oscillations in global MR data (Figure 2, reference [67]). This baseline difference appears similar to an effect observed in conventional ${ }^{1} \mathrm{H}$ SSFP imaging, in which small oscillations in steady-state signal corresponding to RF phase are known to cause a type of imaging artifacts referred to as $N / 2$ ghosts. The $N / 2$ 
ghost artifacts can be removed by repeating phase-encode lines in each RF phase position $( \pm \theta)$ and averaging them before taking a Fourier transform to create the image.

The source of these RF phase-dependent oscillations is traditionally believed to be eddy currents produced by the imaging gradients [68]. However, as no gradient pulses were applied in our experiment, eddy currents due to gradients cannot be the cause of the altered steady state we observe. It has been suggested that imperfection in the excitation RF pulses, such as a phase difference between pulses not equal to $180^{\circ}$, can result in oscillations in the steady state [69].

As outlined in Section 2.5, the ADC value is typically calculated from the ratio of two consecutive MR acquisitions, one with diffusion-weighting and one without. Thus, a baseline difference in signal levels correlated with alternating RF phase angles would compromise our ability to extract an accurate ADC value from a diffusion-weighted SSFP pulse sequence. To better understand this potential confounder, we performed additional tests of the non-diffusion-weighted SSFP pulse sequence to investigate the size and dependence of the baseline asymmetry.

As shown in Figure 4.10c, the steady state ratio of consecutive measurements in a system with a baseline asymmetry alternates between values above and below one. The convention for the results presented below is to only show one of these ratios, red points divided by blue points. This gives a ratio of less than one for RF phase set to $0^{\circ} / 180^{\circ}$. The pulse sequence used for our next series of tests had the following default parameters: $\mathrm{TR}=2.1 \mathrm{~ms}, \theta=4^{\circ}$, RF pulse duration $=100 \mu \mathrm{s}$, and RF phase $=0^{\circ} / 180^{\circ}$. We varied each of these parameters separately to see the dependence of the baseline asymmetry on each. We performed these tests using a hyperpolarized helium-3 phantom on the 1.5 Tesla MRI scanner and using a water phantom on the $1.5 \mathrm{~T}$ and $3 \mathrm{~T}$ scanners. Figure 4.11 shows the ratio of the steady 

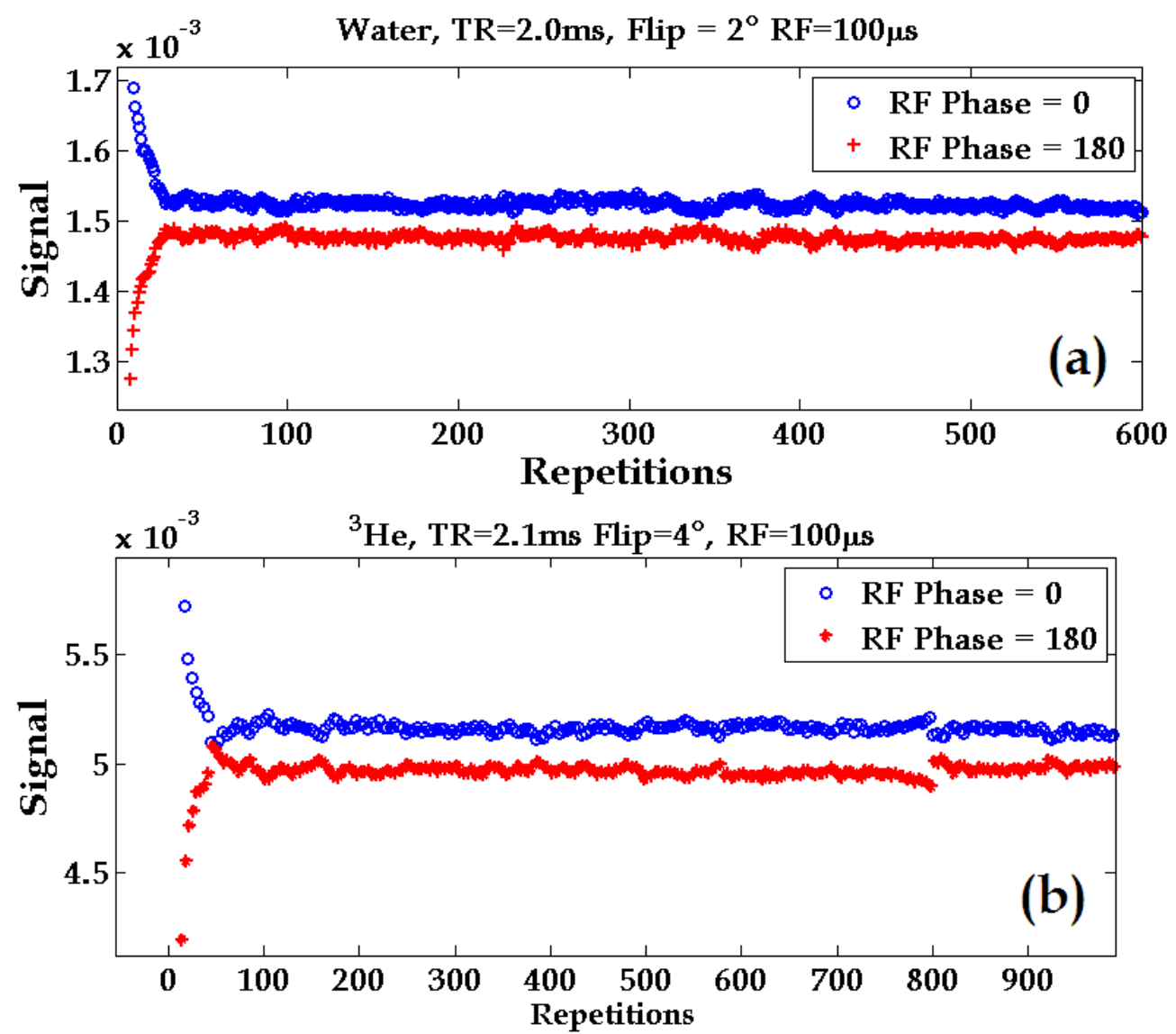

$\mathrm{TR}=2.1 \mathrm{~ms}, \mathrm{Flip}=4^{\circ}, \mathrm{RF}=100 \mu \mathrm{s}$

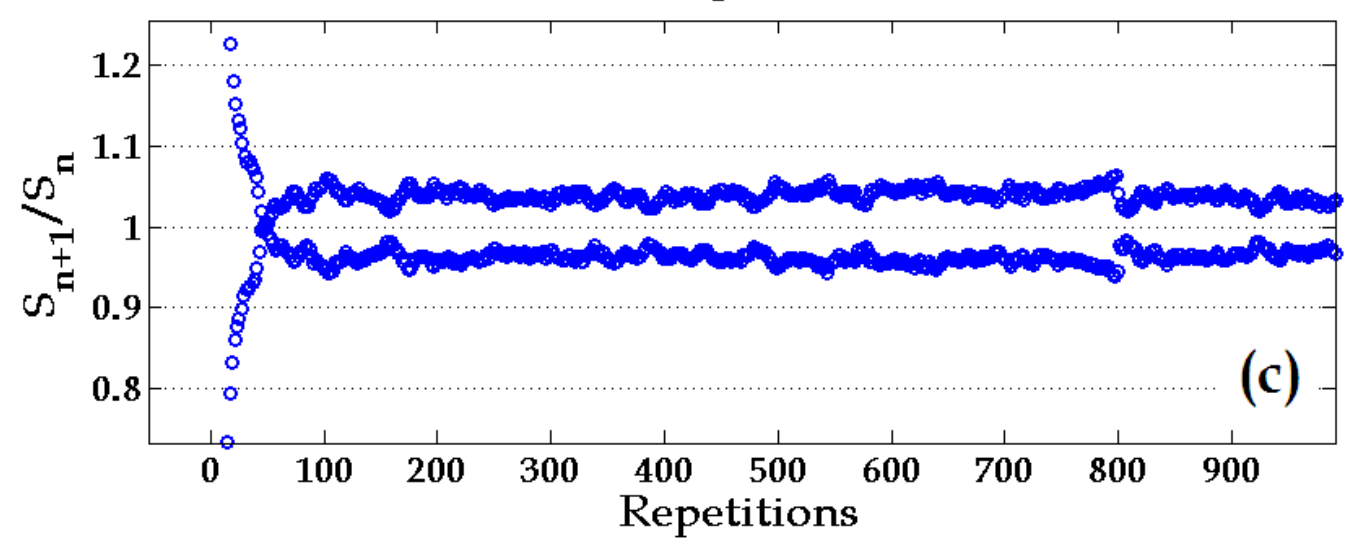

Figure 4.10: Raw global SSFP data from (a) water and (b) hyperpolarized ${ }^{3} \mathrm{He}$. (c) Ratio of consecutive signals from helium data in (b). 
state signal as function of (a) RF phase angle, (b) RF pulse duration, (c) TR, and (d) flip angle.

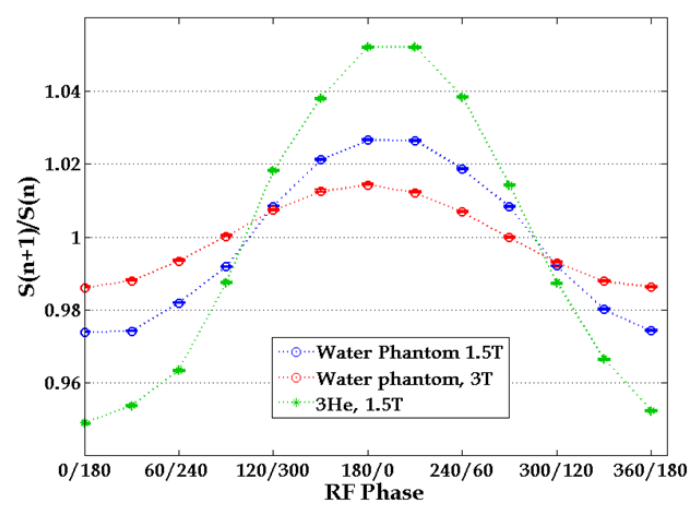

(a) Vary RF Phase Angle

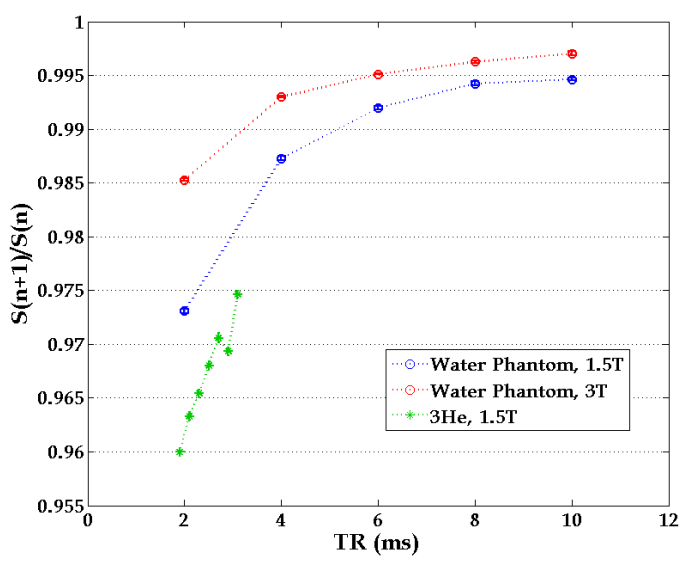

(c) Vary TR

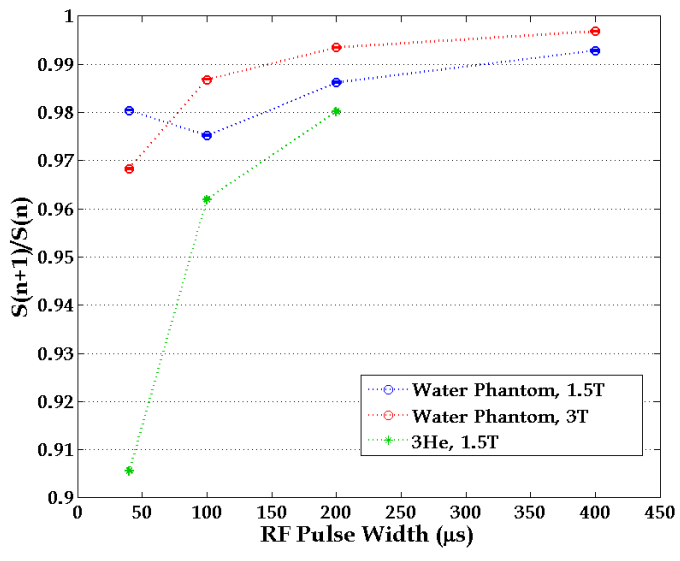

(b) Vary RF Duration

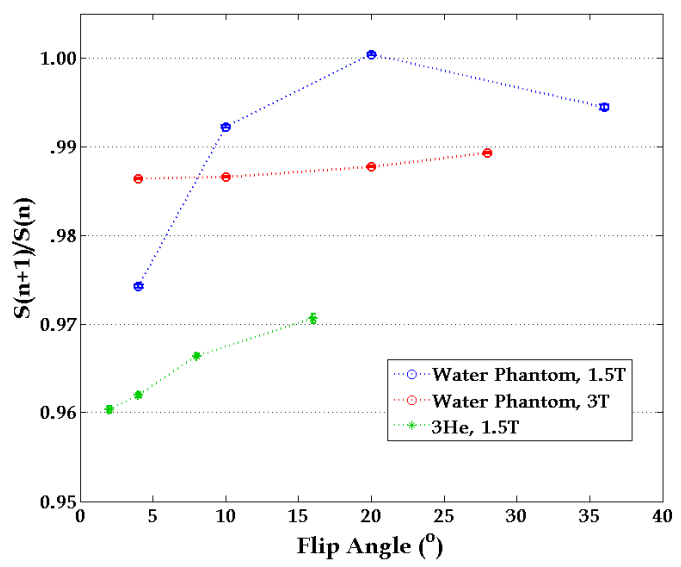

(d) Vary $\theta$

Figure 4.11: Steady-state ratio baseline asymmetry vs (a) RF phase, (b) RF duration, (c) $\mathrm{TR}$, and (d) $\theta$. Data was taken in a water phantom on both the $1.5 T$ and $3 T$ scanners, and in a hyperpolarized ${ }^{3} \mathrm{He}$ phantom at $1.5 \mathrm{~T}$. Unless noted: $\mathrm{TR}=$ 2.1 $\mathrm{ms}, \theta=4^{\circ}$, RF pulse duration $=100 \mu s$, and RF phase $=0^{\circ} / 180^{\circ}$.

In all three of the phantom-scanner tests we performed, the size of the baseline asymmetry has clearly depends on each of the varied parameters. As the RF phase angle (a) changes, the flip-angle axis of rotation is being varied in the $x^{\prime} y^{\prime}-$ plane. Over the course of the $360^{\circ}$ cycle, the rotation axis changes from about $\hat{x}^{\prime}\left(\mathrm{RF}\right.$ phase $\left.=0^{\circ}\right) \rightarrow \hat{y}^{\prime}\left(\mathrm{RF}\right.$ phase $\left.=90^{\circ}\right) \rightarrow-\hat{x}^{\prime}\left(\mathrm{RF}\right.$ phase $\left.=180^{\circ}\right) \rightarrow-\hat{y}^{\prime}(\mathrm{RF}$ 
phase $\left.=270^{\circ}\right) \rightarrow \hat{x}^{\prime}\left(\right.$ RF phase $\left.=360^{\circ}\right)$. Our experiment shows the measured signal ratio goes from less than one to greater than one and back to its initial value. The angular dependence is slightly different for the two scanners (1.5 $\mathrm{T}$ and $3 \mathrm{~T})$, as evidenced by the different zero-crossing for the $3 \mathrm{~T}$ water data compared to both the helium-3 and water data at $1.5 \mathrm{~T}$. It appears that the baseline asymmetry can be minimized by careful selection of the RF phase angles to the zero-crossing, located at approximately $100^{\circ}$ and $280^{\circ}$ for the $1.5 \mathrm{~T}$ MR scanner.

As for the other parameter spaces we explored, the regimes in which the asymmetry is the smallest are also regimes in which it is less desirable to operate when using hyperpolarized magnetization. Increasing RF pulse duration (b) or TR (c) does reduce the size of the asymmetry, however increased TR would lead to an increase in the effects of off-resonant magnetization on the signal. The use of large flip angles (d) also seems to reduce the baseline asymmetry, but large values of $\theta$ could quickly exhaust the signal available from the hyperpolarized gas.

In summary, we measured the baseline difference in SSFP signal levels due to RF phase on two different MRI scanners and using several different RF coils. Although we were unable to determine the origin of the baseline difference, by characterizing the behavior of the asymmetry we were able to devise a strategy for minimizing its effect on our steady state ratio, which is detailed in Section 5.4. 


\subsection{Diffusion-weighted SSFP with Hyperpolarized Mag- netization}

Although this thesis presents the first application of SSFP to hyperpolarized gas diffusion MR, SSFP diffusion-weighted pulse sequences have been implemented for conventional MR, primarily for use in the brain [70-72]. McNab and Miller provide a thorough review of the technique in reference [73]. The most common method of inducing diffusion attenuation in an SSFP pulse sequence is to apply a single diffusion gradient lobe during one TR period, and apply the rephasing gradient lobe during a different TR period several RF excitations later. The large temporal separation of the two gradient lobes is necessary to generate measurable diffusion attenuation, due to the very low diffusion coefficient of water. However, the coherent nature of the SSFP pulse sequence means that each RF excitation converts some fraction of the existing longitudinal magnetization into transverse magnetization and vice versa. Since only the transverse component of the magnetization is affected by the diffusion-sensitizing gradient pulses, and the magnetization in a given sampling region is continually being split along different "pathways" that spend different amounts of time in the transverse plane, there is no universal diffusion time $\Delta$. The diffusion gradient lobes do not have a well-defined $b$ value, therefore it is difficult to extract ADC measurements from this type of diffusionweighted SSFP pulse sequence.

The diffusion coefficient of helium-3 gas is several orders of magnitude larger than that of liquid water (1.1), which means that ample diffusion attenuation can be achieved by a single bipolar gradient contained in one TR period. By being able to induce the diffusion attenuation within one TR period, we can incorporate diffusion sensitization into an SSFP pulse sequence and still have a well-defined 
diffusion time and b-value, which should allow us to extract quantitative ADC measurements. Furthermore, the SSFP pulse sequence should enhance our ability to measure small diffusion attenuations at short diffusion times, as the signal size is larger (and thus has higher SNR) than in an incoherent diffusion-weighted pulse sequence.

We explored four methods of inducing diffusion sensitization in the SSFP se-

quence: (1) apply a bipolar diffusion gradient after every RF pulse and measure the signal before and after the gradient, (2) apply the diffusion gradient after every other RF pulse and compare the signal measured in diffusion-weighted TR periods to non-diffusion-weighted TR periods, (3) apply the diffusion gradient after every third RF pulse and compare the signals measured in the other two TR periods, and (4) apply the diffusion gradient in either the first half or the second half of each TR period and compare the signal measured at the center of each TR period. Each of these methods produces a different effect on the steady-state magnetization, and has advantages and drawbacks for extracting accurate ADC measurements which are explored below.

\subsubsection{Symmetric Diffusion-Weighted SSFP}

The first method we explored for measuring hyperpolarized gas diffusion with an SSFP pulse sequence consists of applying a bipolar diffusion gradient in each TR period. The signal is sampled both before and after the diffusion gradient is applied, and the ratio of the two signal levels is expected to be proportional to the product of the diffusion attenuation and transverse relaxation. The magnetization immediately after the $\mathrm{n}$-th RF pulse is referred to as $M_{+}(n)$. The magnetization evolves throughout the TR window to just before the next RF pulse, where it is 
labeled $M_{-}(n+1)$. To extract a diffusivity measurement, we assume:

$$
M_{-}(n+1)=M_{+}(n) e^{-\frac{T R}{T_{2}}} e^{-b D}
$$

We refer to this pulse sequence as the "Symmetric" diffusion-weighted SSFP pulse sequence, as there is the same amount of signal attenuation in every TR period, producing a steady state magnetization vector that flips symmetrically about the $\hat{z}$ axis, similar to the illustration in Figure 4.3. Because the same amount of relaxation and diffusion attenuation occurs in every TR period, this pulse sequence design maintains a steady state in which the ratio of consecutive pulses is a constant. In order to extract an ADC measurement from the steady state ratio of $M_{-}(n+$ 1) $/ M_{+}(n)$ given in Equation 4.27 , the transverse relaxation must be accounted for by making an independent measurement of $T_{2}$. In order to determine if this method can produce accurate ADC measurements, we simulated the evolution of hyperpolarized magnetization under the influence of the Symmetric diffusionweighted SSFP pulse sequence. Diffusion attenuation was incorporated into the relaxation matrix of Equation 4.5 by replacing $E_{2} \rightarrow E_{2} * E_{D}=e^{-\frac{T R}{T_{2}}} e^{-b D}$. We simulated the magnetization evolution for several values of diffusion attenuation $b D$ using $T R=2 \mathrm{~ms}, T_{1}=20 \mathrm{~s}, T_{2}=100 \mathrm{~ms}$, and $\theta=5^{\circ}$.

Figure 4.12 shows the results of the magnetic simulation versus $b D$. As expected, the ratio $M_{-}(n+1) / M_{+}(n)$ is lower than the diffusion attenuation by a constant factor due to $T_{2}$-decay. When this transverse decay is factored out through an independent measurement, the ratio can be used to calculate an ADC.

An important feature for any diffusion-weighted SSFP pulse sequence is how the ADC measurement is affected by the presence of off-resonant magnetization. As mentioned previously, substantial magnetic field variations are present in the lung due to the magnetic susceptibility differences at the air/tissue interfaces. The 


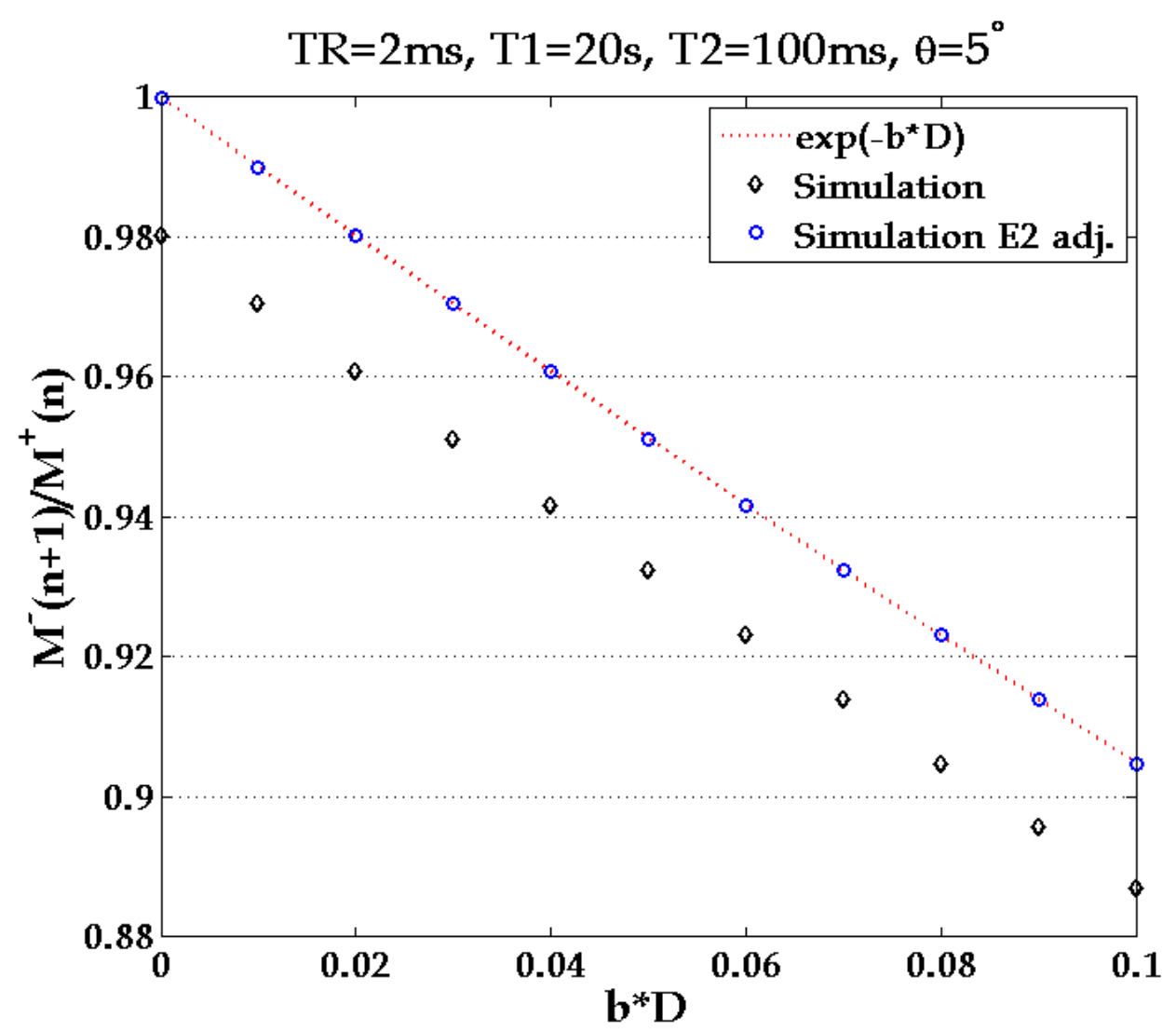

Figure 4.12: Plot of signal ratio $M_{-}(n+1) / M_{+}(n)$ resulting from the simulation of Symmetric diffusion SSFP vs applied diffusion attenuation. The signal ratio is equal to the diffusion attenuation when adjusted for transverse relaxation.

signal measured in an SSFP pulse sequence contains both transverse and longitudinal magnetization recycled from the previous RF pulses. In the presence of magnetic field variations, the previous transverse component will be experiencing refocusing throughout the TR period, in addition to relaxation due to $T_{2}$ and attenuation due to diffusion gradient. In contrast, the longitudinal component will only be declining due to transverse relaxation and diffusion attenuation, not refocusing. The interaction between these different signal evolutions has the potential to affect the signal ratio used for ADC measurement.

We added the ability to simulate off-resonant spins to our simulation of magnetization evolution during an SSFP pulse sequence in order to determine if this 
method of measuring diffusion attenuation is robust in an environment with offresonant magnetization. Instead of a single vector representing the magnetization being measured $M$, a matrix of 101 vectors $M_{j}$ were acted upon by the relaxation and rotation matrices. Each vector was initialized to have only longitudinal magnetization, and was acted on by the rotation matrix as described before. During the transverse evolution, each of the vectors $M_{j}$ was rotated in the transverse plane through an angle of $\beta_{j} / 2$ over each half of the TR period in addition to experiencing transverse relaxation. Two cases of off-resonant magnetization were considered, off-resonant precession due to macroscopic magnetic field variations and precession due to microscopic magnetic field variations. To represent a frequency mismatch due to macroscopic field inhomogeneities, the same $\beta$ value was applied to all of the vectors, $\beta_{j}=\beta$. The effect of microscopic field variations was simulated by rotating the vectors by a collection of $\beta_{j}$ values centered about zero, ranging from $+\beta_{\max }$ to $-\beta_{\max }, \sum_{j=1}^{101}-\beta_{\max }+\frac{j-1}{50}$. In both cases, at the end of the simulation the magnetization was averaged over all of the vectors $M_{j}$.

In the case of a single off-resonance precession angle $\beta$ experienced by every vector, the simulation shows that the ratio of $M_{-}(n+1) / M_{+}(n)$ is proportional to the diffusion attenuation, and thus can be used to calculate an ADC if $T_{2}$ is known. However, Figure 4.13 shows the resulting ratio if there are multiple spins with different values of $\beta$. The ratio is no longer proportional to diffusion attenuation and therefore cannot be used to calculate an ADC. These results can be understood as follows: in the single- $\beta$ case the net transverse magnetization rotates about the $\hat{z}$ axis during the TR period, but otherwise maintains phase coherence and thus the signal magnitude is preserved. In the multiple $\beta$ case, the signal is reduced at the end of the TR period due to loss of phase coherence, as the net signal is a summation over vectors that have each precessed a different amount. Therefore 
the signal has decreased more than by the diffusion attenuation alone, reducing the measured ratio as shown in Figure 4.13 and resulting in an overestimation of the ADC.

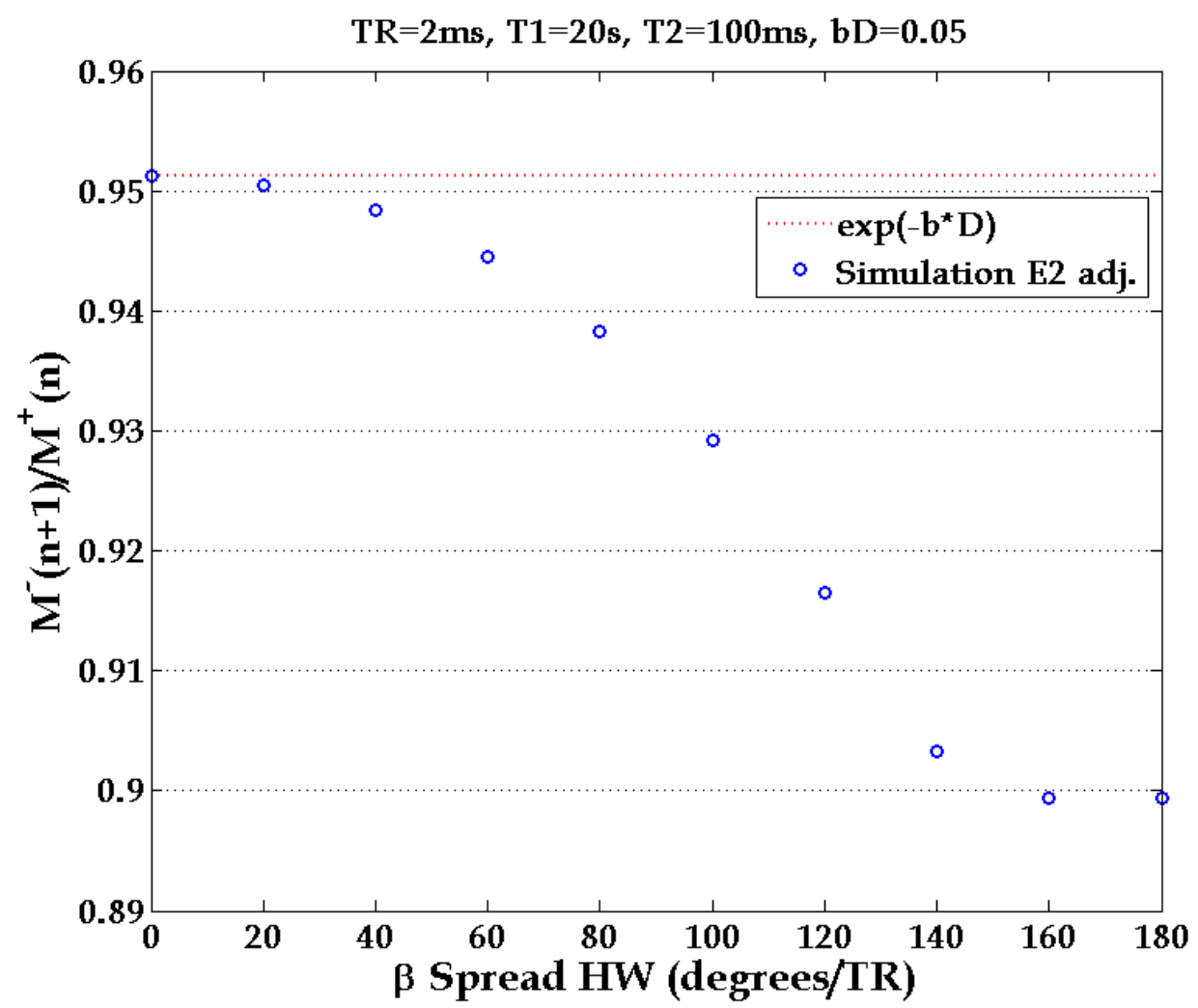

Figure 4.13: Plot of signal ratio $M_{-}(n+1) / M_{+}(n)$ resulting from the simulation of Symmetric diffusion SSFP vs increasing spread of off-resonance precession angle $\beta$. The ratio deviates from the applied diffusion attenuation as a larger spread of off-resonant precession angles are averaged together.

Thus we see that the Symmetric diffusion-weighted SSFP is a straight forward way to generate diffusion-weighting, but it is not well-suited to making ADC measurements in the lung. Furthermore, the comparison of signals measured at different times in the TR period requires in an independent measurement of transverse relaxation $T_{2}$ in order to calculate an ADC from the signal ratio. In an incoherent pulse sequence, this can be achieved by omitting diffusion-sensitizing gradient in every other TR period and measuring the signal ratio in the absence of diffusion 
attenuation. However, it will be seen in the next section that in a coherent pulse sequence, alternating TR periods with and without diffusion attenuation leads to a highly asymmetric steady state which further complicates the measurement.

\subsubsection{Asymmetric Diffusion-Weighted SSFP}

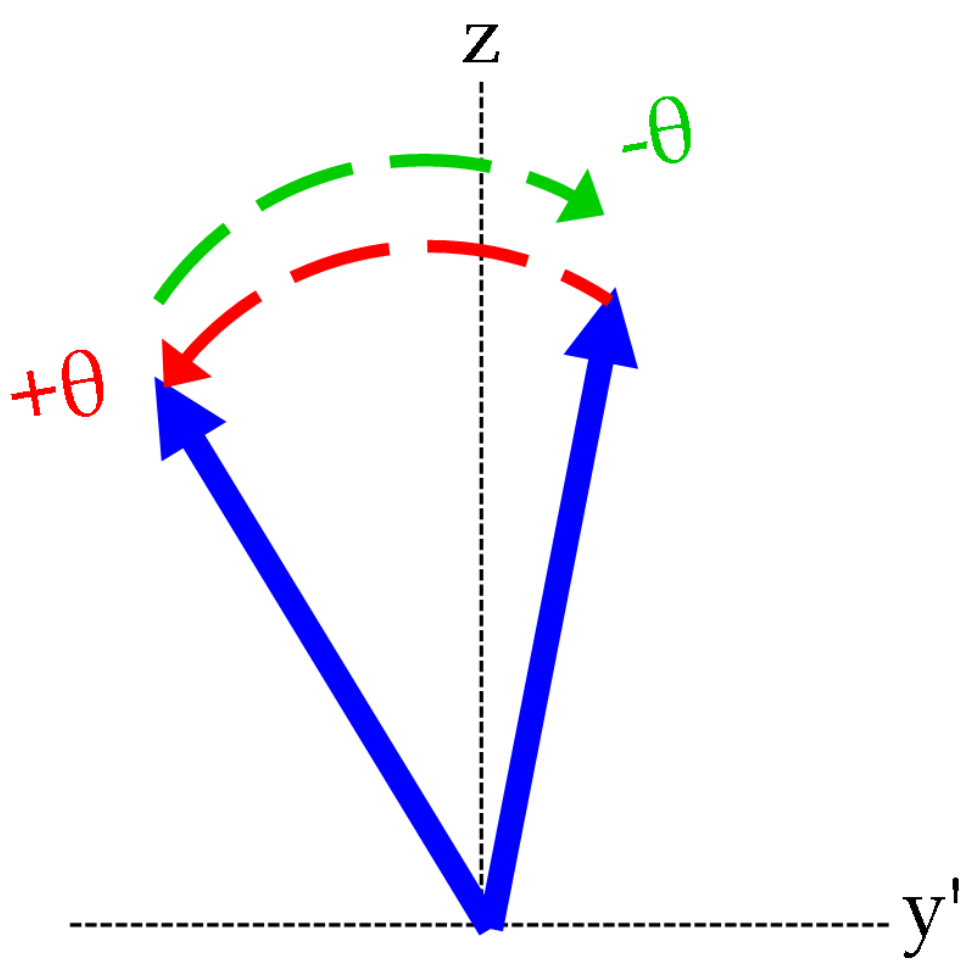

Figure 4.14: In steady state, the magnetization vector alternates asymmetrically. The relaxation behavior has been suppressed in this picture.

The next method we developed for introducing diffusion sensitization into the SSFP pulse sequence mimics the way in which diffusion measurements are made with incoherent pulse sequences, by alternating acquisitions with and without diffusion gradients. However, because the sign of the flip angle also alternates every acquisition, the diffusion attenuation occurs only when the (on-resonance) transverse magnetization vector is on one side of the $\hat{x}^{\prime}$ axis. Since the diffusion attenu- 
ation is applied asymmetrically with respect to the axis of symmetry as shown in Figure 4.14, we refer to this method as the "Asymmetric" diffusion-weighted SSFP pulse sequence.

The simulated magnetization evolution of the Asymmetric diffusion-weighted SSFP pulse sequence is plotted in Figure 4.15. The asymmetric signal attenuation results in a very different steady state than the symmetric case shown in Figure 4.5. Our simulation shows that in this steady state the magnitude of the transverse magnetization is alternating between two levels, although in the hyperpolarized case both levels are decreasing (Figure 4.15a). Figure 4.15b shows the simulated magnetization over four consecutive TR periods after the system has reached steady state. The ratio of the two signal levels is constant at steady state. This ratio depends on the balance between asymmetric magnetization attenuation (due to diffusion) and symmetric attenuation (due to relaxation).

Our simulation indicates that the steady-state signal ratio $M_{y^{\prime}}(n+1) / M_{y^{\prime}}(n)$ can actually be much lower than the signal ratio between diffusion-weighted and non-diffusion weighted signals measured with an incoherent diffusion-weighted pulse sequence:

$$
R_{\text {Incoherent }}=\frac{S(D W)}{S(N D W)}=e^{-b * D},
$$

where $S(D W)$ is the signal from a TR period which contains a bipolar diffusion gradient, and $S(N D W)$ is the signal a TR period with no diffusion gradient. The degree to which $M_{y^{\prime}}(n+1) / M_{y^{\prime}}(n)$ in the Asymmetric diffusion-weighted SSFP pulse sequence is less than $e^{-b * D}$ depends on the relative size of the symmetric attenuation (due to $T_{1}, T_{2}$, and $\theta$ ) compared to the diffusion attenuation. The potential for enhanced sensitivity to diffusion made the Asymmetric diffusion-weighted SSFP pulse sequence attractive for making measurements at very short diffusion times, where the achievable diffusion attenuation is small due to scanner gradient 


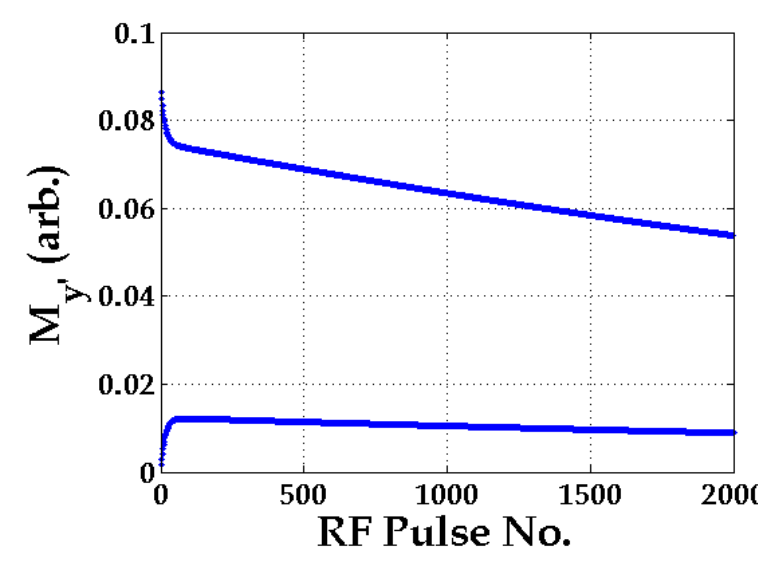

(a) $M_{y^{\prime}}$ vs RF pulse

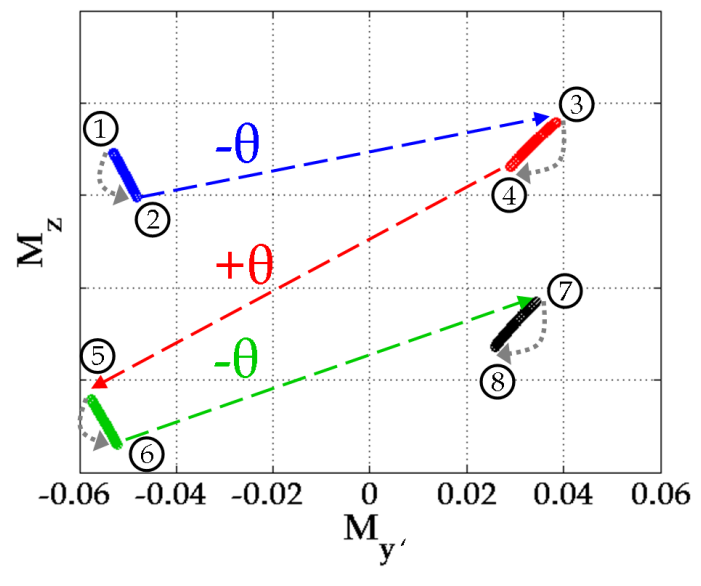

(b) $M_{y^{\prime}}$ vs $M_{z}$

Figure 4.15: Simulated magnetization (a) versus RF pulse and (b) components over several TR periods for hyperpolarized media and an asymmetric diffusionweighted SSFP pulse sequence with TR $=2 m s, T_{1}=20 s, T_{2}=50 m s$, and $\theta=5^{\circ}$. In (a) the signal alternates between the two levels after each RF pulse. In (b)The magnetization evolves sequentially from position 1 to position 8 , and the dashed lines represent RF excitation rotation while the dotted gray lines represent relaxation.

limitations. However, extracting quantitative ADC measurements from the signal ratio $M_{y^{\prime}}(n+1) / M_{y^{\prime}}(n)$ is very complicated as the expression for the ratio will depend on several unknown parameters in addition to $D$. We will derive the steady state ratio $M_{y^{\prime}}(n+1) / M_{y^{\prime}}(n)$ for the Asymmetric diffusion-weighted SSFP pulse sequence, and explore how the ratio is affected by the presence of off-resonance magnetization.

The simulations of the symmetric application of diffusion gradients in the previous section illustrated the importance of measuring the signal at $t=T R / 2$. Figure 4.16 shows the timing of the Asymmetric diffusion-weighted SSFP pulse sequence. The diffusion gradients are applied before and after the data acquisition to maximize the applied diffusion attenuation while maintaining a centered data acquisition. To incorporate diffusion attenuation into our equations and simula- 


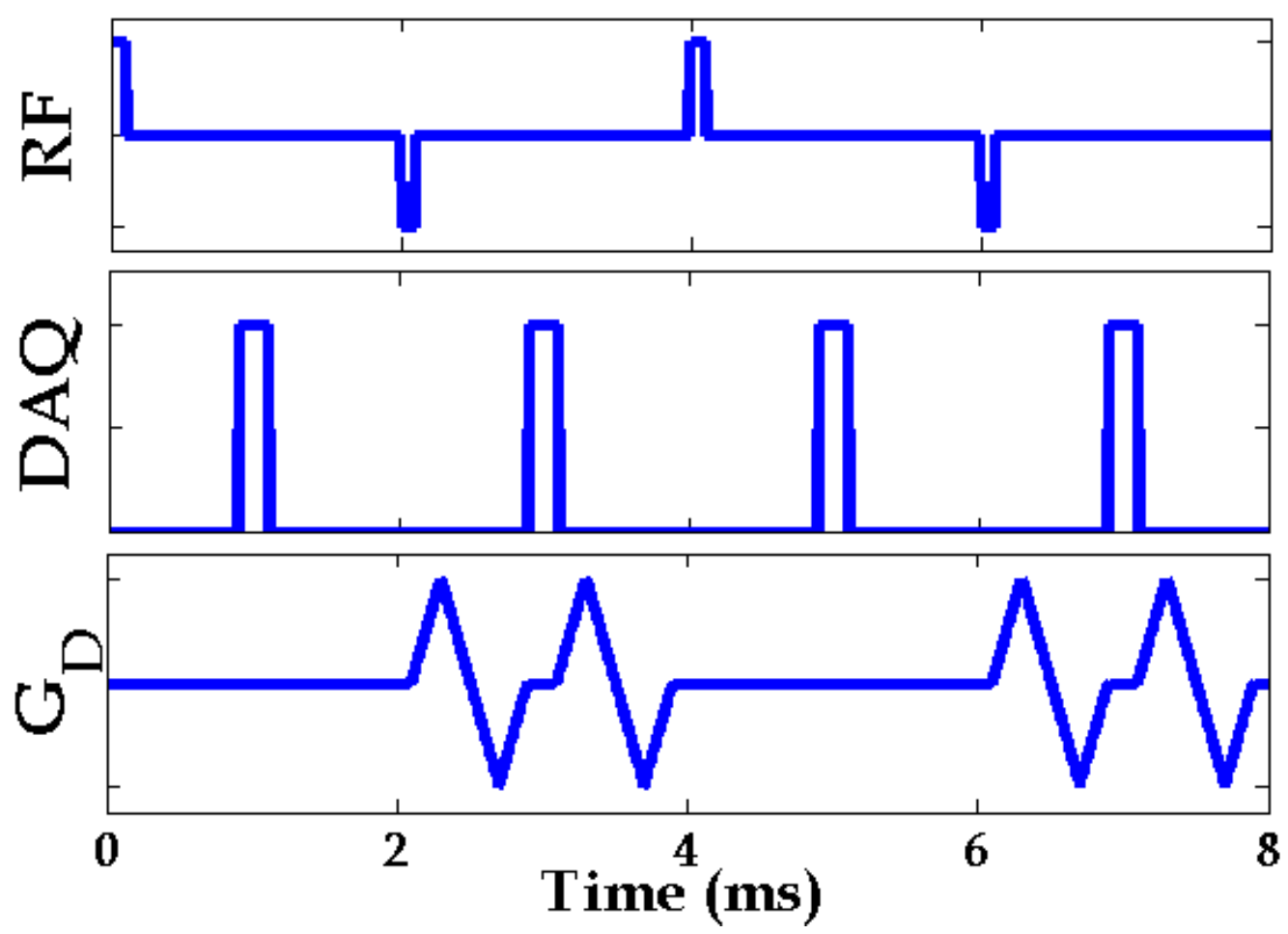

Figure 4.16: Asymmetric diffusion-weighted SSFP pulse sequence diagram. Diffusion gradients $\left(G_{D}\right)$ are applied in every other TR window both before and after the $\mathrm{DAQ}$, in order to measure the signal at $t=T R / 2$.

tion, we model it as an additional transverse relaxation:

$$
E_{D} \equiv e^{-b * D}, \quad \Gamma_{D-1 / 2}=\left(\begin{array}{ccc}
\sqrt{E_{2} E_{D}} & 0 & 0 \\
0 & \sqrt{E_{2} E_{D}} & 0 \\
0 & 0 & \sqrt{E_{1}}
\end{array}\right) \text {. }
$$

The progression of the magnetization is modified from the non-diffusion weighted 
case as follows:

$$
\begin{array}{r}
\mathbf{M}(n+1)=\Gamma_{D-1 / 2} * R_{-}(\theta) * \Gamma_{\text {half }} * \mathbf{M}(n), \\
\mathbf{M}(n+2)=\Gamma_{\text {half }} * R_{+}(\theta) * \Gamma_{D-1 / 2} * \mathbf{M}(n+1), \\
\text { and } \\
\mathbf{M}(n+3)=\Gamma_{D-1 / 2} * R_{-}(\theta) * \Gamma_{\text {half }} * \mathbf{M}(n+2) .
\end{array}
$$

Due to the asymmetry of the diffusion timing the steady state ratio becomes:

$$
\frac{\mathbf{M}(n+1)}{\mathbf{M}(n)}=\frac{\mathbf{M}(n+3)}{\mathbf{M}(n+2)}
$$

Using this equation, we can solve for the transverse component $M_{y^{\prime}}(n+1) / M_{y^{\prime}}(n)$ in terms of $T_{1}, T_{2}, T R, \theta, b$, and $D$. We begin with some steady state magnetization:

$$
\mathbf{M}(n)=\left(\begin{array}{c}
0 \\
M_{y^{\prime}} \\
M_{z}
\end{array}\right)
$$

Solving for $M(n+1)$ :

$$
\begin{gathered}
\mathbf{M}(n+1)=\Gamma_{D-1 / 2} * R_{-}(\theta) * \Gamma_{\text {half }} * \mathbf{M}(n) \\
=\left(\begin{array}{c}
0 \\
c E_{2} \sqrt{E_{D}} M_{y^{\prime}}-s \sqrt{E_{1} E_{2} E_{D}} M_{z} \\
s \sqrt{E_{1} E_{2}} M_{y^{\prime}}+c E_{1} M_{z}
\end{array}\right) .
\end{gathered}
$$


Now we can solve for the left side of equation 4.34:

$$
\frac{M_{y^{\prime}}(n+1)}{M_{y^{\prime}}(n)}=c E_{2} \sqrt{E_{D}}-s \sqrt{E_{1} E_{2} E_{D}} \frac{M_{z}}{M_{y^{\prime}}} .
$$

We solve for $\frac{M_{z}}{M_{y^{\prime}}}$ by getting expressions for $\mathbf{M}(n+2)$ and $\mathbf{M}(n+3)$ and rearranging Equation 4.34. We determine $\mathbf{M}(n+2)$ :

$$
\begin{gathered}
\mathbf{M}(n+2)=\Gamma_{\text {half }} * R_{+}(\theta) * \Gamma_{\text {Dhalf }} * \mathbf{M}(n+1) \\
=\left(\begin{array}{c}
0 \\
c E_{2} \sqrt{E_{D}} M_{y^{\prime}}(n+1)+s \sqrt{E_{1} E_{2}} M_{z}(n+1) \\
-s \sqrt{E_{1} E_{2} E_{D}} M_{y^{\prime}}(n+1)+c E_{1} M_{z}(n+1)
\end{array}\right)
\end{gathered}
$$

Substituting the values for $\mathbf{M}(n+1)$ from Equation 4.37, we have:

$$
\mathbf{M}(n+2)=\left(\begin{array}{c}
0 \\
\left(c^{2} E_{2}^{2} E_{D}+s^{2} E_{1} E_{2}\right) M_{y^{\prime}}+c s \sqrt{E_{1} E_{2}}\left(E_{1}-E_{2} E_{D}\right) M_{z} \\
c s \sqrt{E_{1} E_{2}}\left(E_{1}-E_{2} E_{D}\right) M_{y^{\prime}}+\left(s^{2} E_{1} E_{2} E_{D}+c^{2} E_{1}^{2}\right) M_{z}
\end{array}\right) .
$$

Now we solve for $\mathbf{M}(n+3)$ :

$$
\begin{gathered}
\mathbf{M}(n+3)=\Gamma_{\text {Dhalf }} * R_{-}(\theta) * \Gamma_{\text {half }} * \mathbf{M}(n+2) \\
=\left(\begin{array}{c}
0 \\
c E_{2} \sqrt{E_{D}} M_{y^{\prime}}(n+2)-s \sqrt{E_{1} E_{2} E_{D}} M_{z}(n+2) \\
s \sqrt{E_{1} E_{2}} M_{y^{\prime}}(n+2)+c E_{1} M_{z}(n+2)
\end{array}\right) .
\end{gathered}
$$


Substituting the values for $\mathbf{M}(n+2)$ from Equation 4.41, we have:

$$
\mathbf{M}(n+3)=\left(\begin{array}{c}
0 \\
\left(c^{3} E_{2}^{3} E_{D} \sqrt{E_{D}}+c s^{2} E_{1} E_{2}^{2} \sqrt{E_{D}}\right) M_{y^{\prime}}+c^{2} E_{2} s \sqrt{E_{1} E_{2} E_{D}}\left(E_{1}-E_{2} E_{D}\right) M_{z} \\
-\left(c s^{2} E_{1} E_{2} \sqrt{E_{D}}\left(E_{1}-E_{2} E_{D}\right) M_{y^{\prime}}+\left(s^{3} E_{1} E_{2} E_{D} \sqrt{E_{1} E_{2} E_{D}}+c^{2} s E_{1}^{2} \sqrt{E_{1} E_{2} E_{D}}\right) M_{z}\right) \\
\left(c^{2} s E_{2}^{2} E_{D} \sqrt{E_{1} E_{2}}+s^{3} E_{1} E_{2} \sqrt{E_{1} E_{2}}\right) M_{y^{\prime}}+c s^{2} E_{1} E_{2}\left(E_{1}-E_{2} E_{D}\right) M_{z} \\
+c^{2} s \sqrt{E_{1} E_{2}}\left(E_{1}^{2}-E_{1} E_{2} E_{D}\right) M_{y^{\prime}}+\left(c s^{2} E_{1}^{2} E_{2} E_{D}+c^{3} E_{1}^{3}\right) M_{z} \\
0 \\
c E_{2} \sqrt{E_{D}}\left(c^{2} E_{2}^{2} E_{D}+s^{2}\left(E_{1} E_{2}-E_{1}^{2}+E_{1} E_{2} E_{D}\right)\right) M_{y^{\prime}} \\
+s \sqrt{E_{1} E_{2} E_{D}}\left(c^{2}\left(E_{1} E_{2}-E_{2}^{2} E_{D}-E_{1}^{2}\right)-s^{2} E_{1} E_{2} E_{D}\right) M_{z} \\
s \sqrt{E_{1} E_{2}}\left(s^{2} E_{1} E_{2}+c^{2}\left(E_{1}^{2}-E_{1} E_{2} E_{D}+E_{2}^{2} E_{D}\right)\right) M_{y^{\prime}} \\
+c E_{1}\left(c^{2} E_{1}^{2}+s^{2}\left(E_{1} E_{2} E_{D}+E_{1} E_{2}-E_{2}^{2} E_{D}\right)\right) M_{z}
\end{array}\right) .
$$

With these expressions, we can substitute into the transverse component of Equation 4.34:

$$
\frac{M_{y^{\prime}}(n+1)}{M_{y^{\prime}}(n)}=\frac{M_{y^{\prime}}(n+3)}{M_{y^{\prime}}(n+2)}
$$

and solve for $\frac{M_{z}}{M_{y^{\prime}}}$ in Equation 4.38. Rearranging our terms in Equation 4.46:

$$
M_{y^{\prime}}(n+1) * M_{y^{\prime}}(n+2)=M_{y^{\prime}}(n+3) * M_{y^{\prime}}(n),
$$

we can substitute in the components of $M_{y^{\prime}}(n+1), M_{y^{\prime}}(n+2)$, and $M_{y^{\prime}}(n+3)$ in 
terms of $M_{y^{\prime}}$ and $M_{z}$ alone:

$$
\begin{gathered}
\left(c E_{2} \sqrt{E_{D}} M_{y^{\prime}}-s \sqrt{E_{1} E_{2} E_{D}} M_{z}\right) \\
*\left(\left(c^{2} E_{2}^{2} E_{D}+s^{2} E_{1} E_{2}\right) M_{y^{\prime}}+c s \sqrt{E_{1} E_{2}}\left(E_{1}-E_{2} E_{D}\right) M_{z}\right) \\
=c E_{2} \sqrt{E_{D}}\left(c^{2} E_{2}^{2} E_{D}+s^{2}\left(E_{1} E_{2}-E_{1}^{2}+E_{1} E_{2} E_{D}\right)\right) M_{y^{\prime}}^{2} \\
+s \sqrt{E_{1} E_{2} E_{D}}\left(c^{2}\left(E_{1} E_{2}-E_{2}^{2} E_{D}-E_{1}^{2}\right)-s^{2} E_{1} E_{2} E_{D}\right) M_{z} M_{y^{\prime}} .
\end{gathered}
$$

We simplify both sides of the expression:

$$
\begin{gathered}
c E_{2} \sqrt{E_{D}}\left(c^{2} E_{2}^{2} E_{D}+s^{2} E_{1} E_{2}\right) M_{y^{\prime}}^{2} \\
+s \sqrt{E_{1} E_{2} E_{D}}\left(c^{2}\left(E_{1} E_{2}-2 E_{2}^{2} E_{D}\right)-s^{2} E_{1} E_{2}\right) M_{z} M_{y^{\prime}} \\
-c s^{2} E_{1} E_{2} \sqrt{E_{D}}\left(E_{1}-E_{2} E_{D}\right) M_{z}^{2} \\
=c E_{2} \sqrt{E_{D}}\left(c^{2} E_{2}^{2} E_{D}+s^{2}\left(E_{1} E_{2}-E_{1}^{2}+E_{1} E_{2} E_{D}\right)\right) M_{y^{\prime}}^{2} \\
+s \sqrt{E_{1} E_{2} E_{D}}\left(c^{2}\left(E_{1} E_{2}-E_{2}^{2} E_{D}-E_{1}^{2}\right)-s^{2} E_{1} E_{2} E_{D}\right) M_{z} M_{y}
\end{gathered}
$$

and rearrange terms:

$$
\begin{aligned}
0 & =c s^{2} E_{1} E_{2} \sqrt{E_{D}}\left(E_{1}-E_{2} E_{D}\right) M_{z}^{2} \\
& +s \sqrt{E_{1} E_{2} E_{D}}\left(c^{2} E_{2}^{2} E_{D}+s^{2} E_{1} E_{2}\right) M_{z} M_{y^{\prime}} \\
& -s \sqrt{E_{1} E_{2} E_{D}}\left(c^{2} E_{1}^{2}+s^{2} E_{1} E_{2} E_{D}\right) M_{z} M_{y^{\prime}} \\
& +c s^{2} E_{1} E_{2} \sqrt{E_{D}}\left(E_{2} E_{D}-E_{1}\right) M_{y^{\prime}}^{2} .
\end{aligned}
$$


Dividing by $M_{y^{\prime}}^{2}$, we have a quadratic equation involving $\frac{M_{z}}{M_{y^{\prime}}}$ :

$$
\begin{aligned}
0 & =c s^{2} E_{1} E_{2} \sqrt{E_{D}}\left(E_{1}-E_{2} E_{D}\right)\left(\frac{M_{z}}{M_{y^{\prime}}}\right)^{2} \\
& +s \sqrt{E_{1} E_{2} E_{D}}\left(c^{2}\left(E_{2}^{2} E_{D}-E_{1}^{2}\right)+s^{2}\left(E_{1} E_{2}-E_{1} E_{2} E_{D}\right)\right) \frac{M_{z}}{M_{y^{\prime}}} \\
& -c s^{2} E_{1} E_{2} \sqrt{E_{D}}\left(E_{2} E_{D}-E_{1}\right) .
\end{aligned}
$$

Solving the quadratic equation for $\frac{M_{z}}{M_{y^{\prime}}}$ :

$$
\frac{M_{z}}{M_{y^{\prime}}}=\frac{-s \sqrt{E_{1} E_{2} E_{D}}\left(c^{2}\left(E_{2}^{2} E_{D}-E_{1}^{2}\right)+s^{2}\left(E_{1} E_{2}-E_{1} E_{2} E_{D}\right)\right) \pm \sqrt{X}}{2 c s^{2} E_{1} E_{2} \sqrt{E_{D}}\left(E_{1}-E_{2} E_{D}\right)}
$$

where

$$
X \equiv s^{2} E_{1} E_{2} E_{D}\left(c^{2}\left(E_{2}^{2} E_{D}-E_{1}^{2}\right)+s^{2}\left(E_{1} E_{2}-E_{1} E_{2} E_{D}\right)\right)^{2}+4 c^{2} s^{4} E_{1}^{2} E_{2}^{2} E_{D}\left(E_{1}-E_{2} E_{D}\right)^{2}
$$

Simplifying the expression for $\mathrm{X}$ :

$$
\begin{array}{r}
X=s^{2} E_{1} E_{2} E_{D}\left[c^{4}\left(E_{2}^{2} E_{D}-E_{1}^{2}\right)^{2}+s^{4}\left(E_{1} E_{2}-E_{1} E_{2} E_{D}\right)^{2}\right. \\
+2 c^{2} s^{2}\left(E_{1} E_{2}^{3} E_{D}-E_{1}^{3} E_{2}-E_{1} E_{2}^{3} E_{D}^{2}+E_{1}^{3} E_{2} E_{D}\right) \\
\left.+4 c^{2} s^{2} E_{1} E_{2}\left(E_{1}^{3} E_{2}-2 E_{1}^{2} E_{2}^{2} E_{D}+E_{1} E_{2}^{3} E_{D}^{2}\right)\right] \\
=s^{2} E_{1} E_{2} E_{D}\left[c^{4}\left(E_{2}^{2} E_{D}-E_{1}^{2}\right)^{2}+s^{4}\left(E_{1} E_{2}-E_{1} E_{2} E_{D}\right)^{2}\right. \\
\left.+2 c^{2} s^{2}\left(E_{1} E_{2}^{3} E_{D}+E_{1}^{3} E_{2}+E_{1} E_{2}^{3} E_{D}^{2}+E_{1}^{3} E_{2} E_{D}-4 E_{1}^{2} E_{2}^{2} E_{D}\right)\right]
\end{array}
$$




$$
\begin{aligned}
= & s^{2} E_{1} E_{2} E_{D}\left[c^{4}\left(E_{2}^{2} E_{D}-E_{1}^{2}\right)^{2}+s^{4}\left(E_{1} E_{2}-E_{1} E_{2} E_{D}\right)^{2}\right. \\
& \left.+2 c^{2} s^{2}\left(\left(E_{1}^{3} E_{2}+E_{1} E_{2}^{3} E_{D}\right)\left(1+E_{D}\right)-4 E_{1}^{2} E_{2}^{2} E_{D}\right)\right] .
\end{aligned}
$$

Now we can insert the expression for $\frac{M_{z}}{M_{y^{\prime}}}$ into Equation 4.38:

$$
\begin{gathered}
\frac{M_{y^{\prime}}(n+1)}{M_{y^{\prime}}(n)}=c E_{2} \sqrt{E_{D}} \\
-s \sqrt{E_{1} E_{2} E_{D}} * \frac{-s \sqrt{E_{1} E_{2} E_{D}}\left(c^{2}\left(E_{2}^{2} E_{D}-E_{1}^{2}\right)+s^{2}\left(E_{1} E_{2}-E_{1} E_{2} E_{D}\right)\right) \pm s \sqrt{E_{1} E_{2} E_{D}} * \sqrt{X}}{2 c s^{2} E_{1} E_{2} \sqrt{E_{D}}\left(E_{1}-E_{2} E_{D}\right)},
\end{gathered}
$$

where

$$
\begin{gathered}
X \equiv c^{4}\left(E_{2}^{2} E_{D}-E_{1}^{2}\right)^{2}+s^{4}\left(E_{1} E_{2}-E_{1} E_{2} E_{D}\right)^{2} \\
+2 c^{2} s^{2}\left(\left(E_{1}^{3} E_{2}+E_{1} E_{2}^{3} E_{D}\right)\left(1+E_{D}\right)-4 E_{1}^{2} E_{2}^{2} E_{D}\right) .
\end{gathered}
$$

Simplifying the expression for $M_{y^{\prime}}(n+1) / M_{y^{\prime}}(n)$ :

$$
\begin{gathered}
\frac{M_{y^{\prime}}(n+1)}{M_{y^{\prime}}(n)}=c E_{2} \sqrt{E_{D}} \\
+s^{2} E_{1} E_{2} E_{D} * \frac{\left(c^{2} \sqrt{E_{D}}\left(E_{2}^{2} E_{D}-E_{1}^{2}\right)+s^{2} \sqrt{E_{D}}\left(E_{1} E_{2}-E_{1} E_{2} E_{D}\right)\right) \mp \sqrt{X}}{2 c s^{2} E_{1} E_{2} \sqrt{E_{D}}\left(E_{1}-E_{2} E_{D}\right)}, \\
\frac{M_{y^{\prime}}(n+1)}{M_{y^{\prime}}(n)}=c E_{2} \sqrt{E_{D}} \\
+\sqrt{E_{D}} * \frac{\left(c^{2}\left(E_{2}^{2} E_{D}-E_{1}^{2}\right)+s^{2}\left(E_{1} E_{2}-E_{1} E_{2} E_{D}\right)\right) \mp \sqrt{X}}{2 c\left(E_{1}-E_{2} E_{D}\right)} .
\end{gathered}
$$


Equation 4.60 describes the ratio of consecutive transverse magnetizations which results from introducing diffusion attenuation into alternating TR periods. The ratio of the next pair of signals $M_{y^{\prime}}(n+2) / M_{y^{\prime}}(n+1)$ would be the reciprocal as the diffusion attenuation switches from numerator to denominator. Equation 4.60 has a complicated dependence on the relaxation, excitation, and diffusion parameters, so it is instructive to plot the result. We simulated the Asymmetric diffusionweighted SSFP pulse sequence on hyperpolarized magnetization, and the results of the simulation are plotted versus $b D$ in Figure 4.17 with Equation 4.60. The minus (-) expression (red stars) again matches the simulation of the magnetization (blue points). The ratio of consecutive signals is much less than the incoherent signal ratio $e^{-b * D}$ (black line) indicating that $\mathrm{ADC}$ maps made using this sequence would have increased contrast compared to conventional incoherent ADC maps. The enhancement of contrast due to diffusion would aid in the measurement diffusion attenuation due to short-time scale gradients.

Due to the complexity of Equation 4.60, extracting an ADC value from data acquired with the Asymmetric diffusion-weighted SSFP pulse sequence requires knowledge of $T_{1}, T_{2}$, and $\theta$. These parameters are not necessarily uniform throughout a sample, which makes the Asymmetric diffusion SSFP pulse sequence impractical for quantitative measurements. However it is a promising option for diffusion-weighted imaging due to the high contrast, which would be useful in comparing healthy and diseased lung areas even if the signal ratio did not translate into an $\mathrm{ADC}$ value.

The behavior of $M_{y^{\prime}}(n+1) / M_{y^{\prime}}(n)$ in the presence of off-resonance magnetization is an important feature to study, as off-resonance magnetization is unavoidable in the lung. We incorporated off-resonance magnetization into our simulation using the same basic methods described in the previous section. The results are 


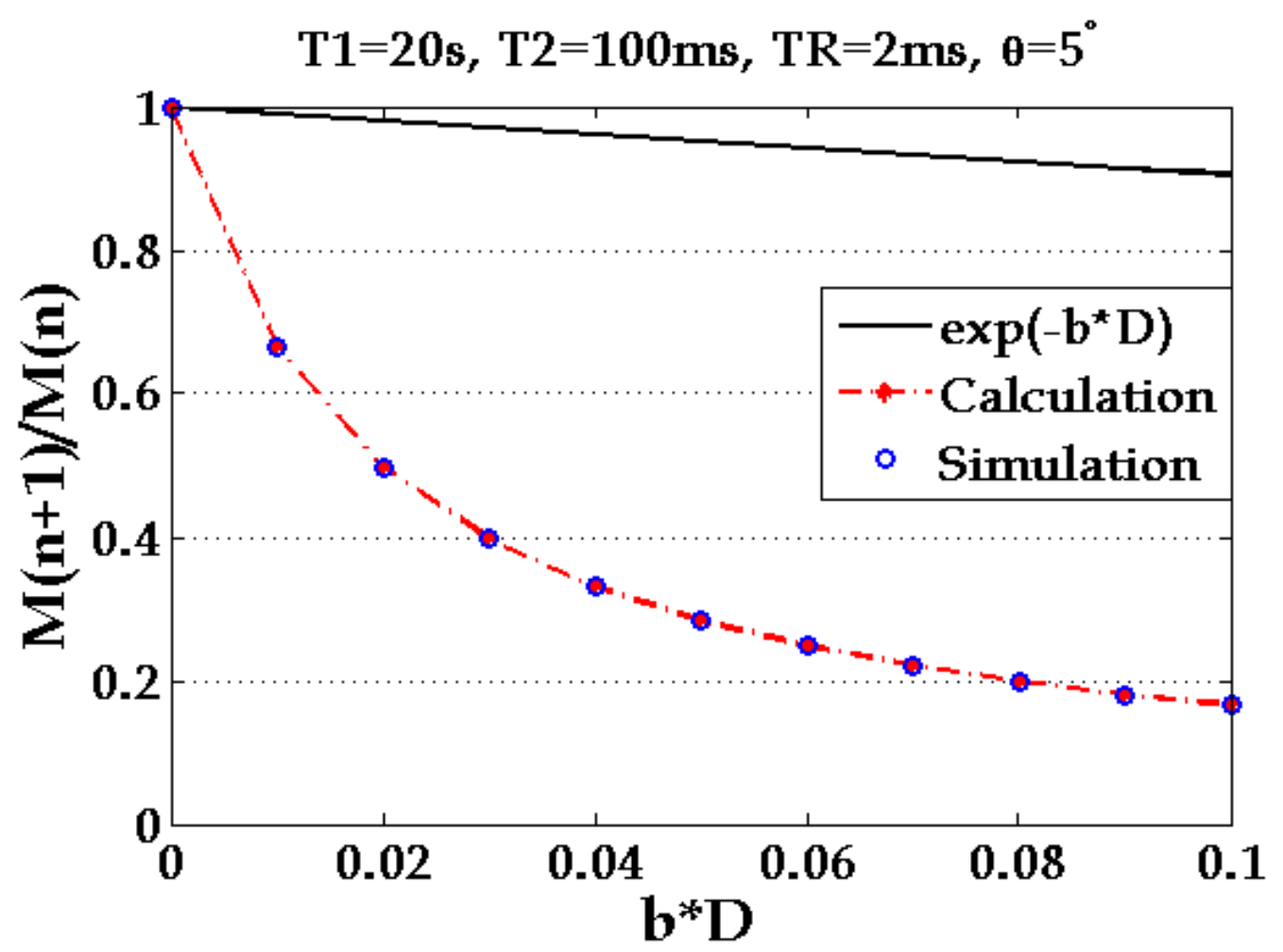

Figure 4.17: Plot of signal ratio resulting from the simulation of Asymmetric diffusion SSFP (blue points), diffusion attenuation (black line), and Equation 4.60 (red line) versus $b^{*} \mathrm{D}$. $T 1=20 \mathrm{~s}, \mathrm{~T} 2=100 \mathrm{~ms}, \theta=5^{\circ}$.

plotted in Figure 4.18a for a single off-resonance precession angle $\beta$, and in Figure $4.25 \mathrm{~b}$ for the superposition of a range of off-resonance precession angles. The simulated ratio values are plotted as blue points, along with the calculated steadystate ratio from Equation 4.60 as the red line, and the incoherent ratio $e^{-b * D}$ as the black line.

These results show that the significant contrast enhancement the occurs for on-resonance magnetization is greatly reduced even for small off-resonant precession angles, and becomes less than the incoherent ratio $e^{-b * D}$ for a single $\beta>$ $10^{\circ}$ per TR. Thus an extremely uniform holding field would be necessary to make this diffusion-weighted pulse sequence work in practice. 


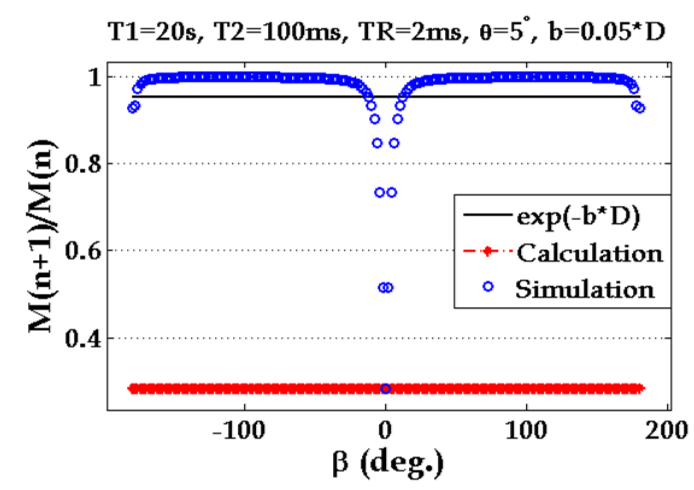

(a) Frequency Offset

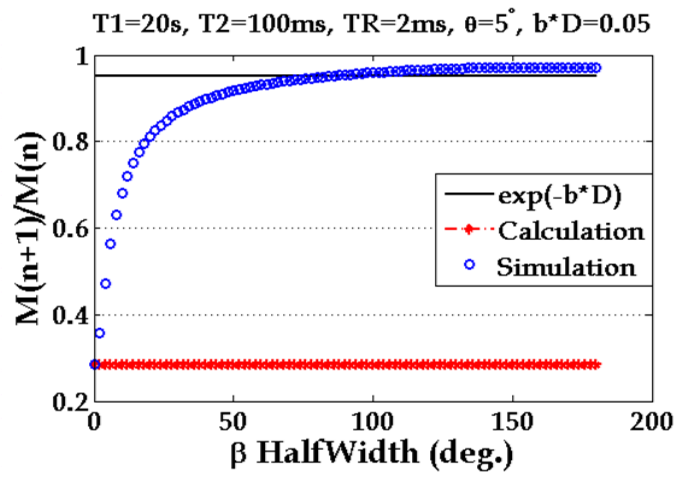

(b) Frequency Spread

Figure 4.18: Plot of signal ratio resulting from the simulation of Asymmetric diffusion SSFP (blue points), $e^{-b * D}$ (black line), and equation 4.60 (red line) versus off resonance width, in degrees per TR. The parameters used in simulation and calculation were: $T_{1}=20 \mathrm{~s}, T_{2}=100 \mathrm{~ms}, \theta=5^{\circ}$, TR $=2 \mathrm{~ms}$. (a) shows the effect of a single $\beta$, (b) shows effect of averaging over a collection of $\beta$ values. 


\subsubsection{Group of Three Diffusion-Weighted SSFP}

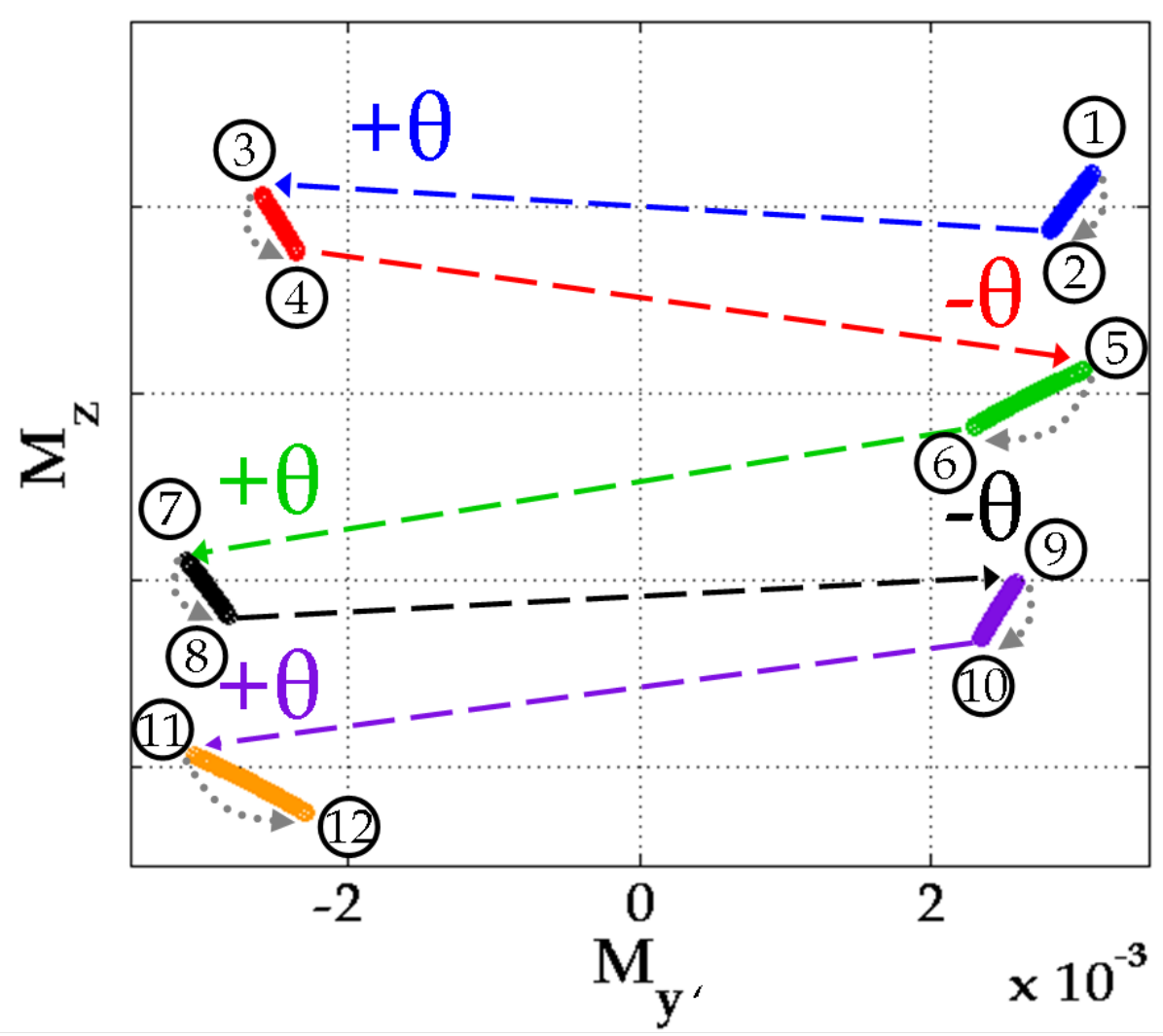

Figure 4.19: Simulated magnetization for the Group of Three diffusion-weighted SSFP pulse sequence with TR $=2 m s, T_{1}=20 s, T_{2}=50 m s$, and $\theta=5^{\circ}$. The magnetization evolves sequentially from position 1 to position 12, and the dashed lines represent RF excitation rotation while the dotted gray lines represent relaxation.

The next method we explored for quantitative obtaining quantitative measurements of ADC values involves applying the diffusion gradient after every third pulse and measuring the signal in the TR periods that do not contain diffusion gradients. Figure 4.19 shows the magnetization evolution through six TR periods. The periods between $1 / 2,3 / 4,7 / 8$, and $9 / 10$ experience relaxation only, whereas the periods between 5/6 and 11/12 experience both relaxation and diffusion attenuation. The steady state that develops has constant signal ratio between the TR 
periods in which there was no diffusion attenuation:

$$
\frac{S(3 \rightarrow 4)}{S(1 \rightarrow 2)}=\frac{S(9 \rightarrow 10)}{S(7 \rightarrow 8)}
$$

where $S(1 \rightarrow 2)$ is the signal measured between time points 1 and 2 , etc. The size of the steady state ratio is again determined by a combination of diffusion and relaxation parameters. The signal evolution during the TR periods displayed on the graph with the larger transverse attenuation $(5 / 6$ and $11 / 12)$ cannot actually be measured, as the magnetization is dephased during the application of the diffusion gradients.
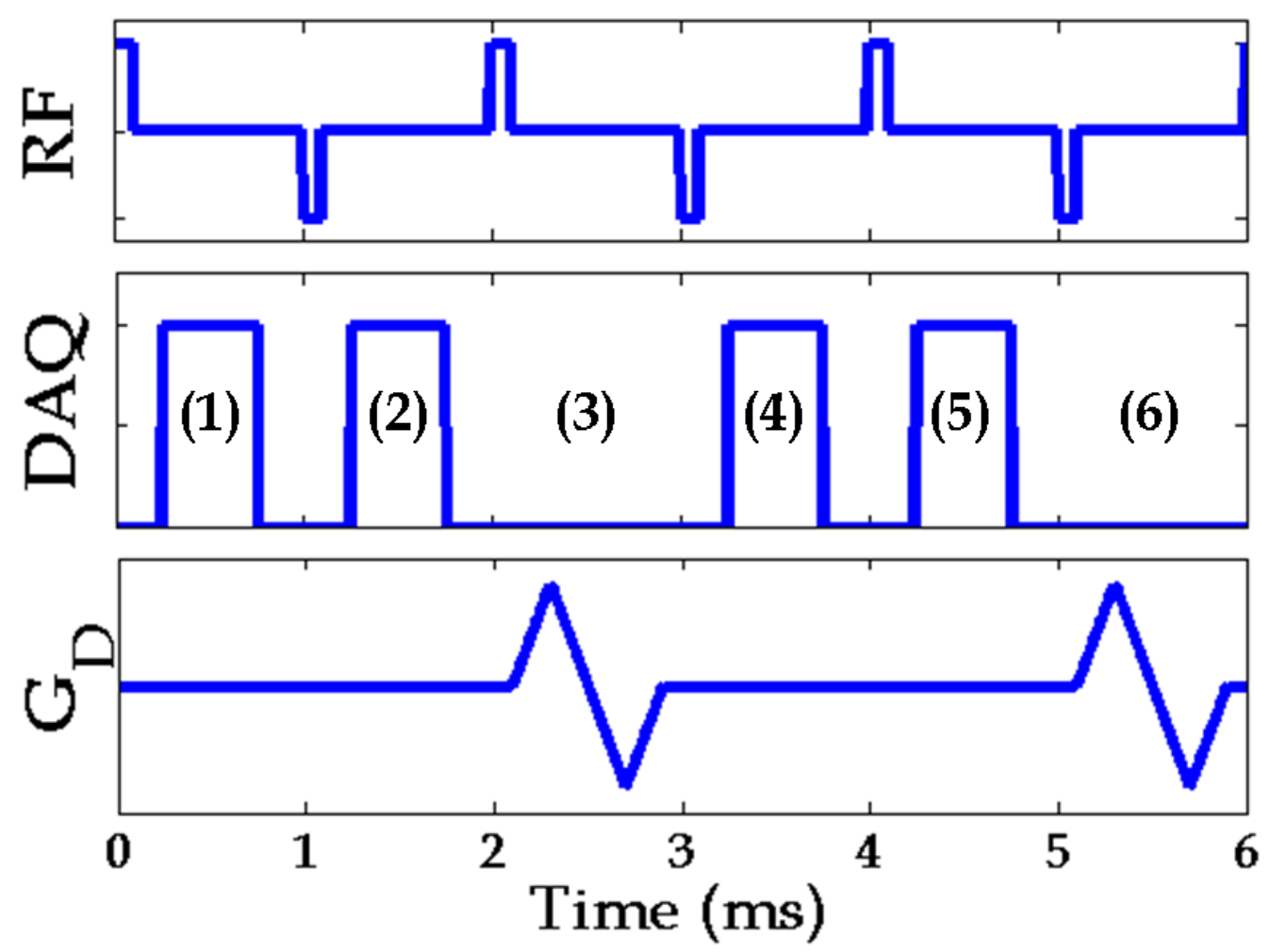

Figure 4.20: Group of Three diffusion-weighted SSFP pulse sequence diagram. Diffusion gradients $\left(G_{D}\right)$ are applied in every third TR window (3 and 6), and the signal ratio of the other two windows $(2 / 1$ and $5 / 4)$ reaches a steady state that is strongly weighted by diffusion.

To be able to extract diffusivity measurements from the measured signal ratio 
in this pulse sequence, we must first derive the expression for the steady state signal ratio $M_{y^{\prime}}(n+1) / M_{y^{\prime}}(n)$, and then examine the behavior of the ratio in the presence of off-resonant magnetization.

The progression of the magnetization as measured at TR/2 is as follows:

$$
\begin{array}{r}
\mathbf{M}(n+1)=\Gamma_{\text {half }} * R_{-}(\theta) * \Gamma_{\text {half }} * \mathbf{M}(n), \\
\mathbf{M}(n+3)=\Gamma_{\text {half }} * R_{-}(\theta) * \Gamma_{D} * R_{+}(\theta) * \Gamma_{\text {half }} * \mathbf{M}(n+1), \\
\text { and } \\
\mathbf{M}(n+4)=\Gamma_{\text {half }} * R_{+}(\theta) * \Gamma_{\text {half }} * \mathbf{M}(n+3),
\end{array}
$$

where $\mathbf{M}(n+2)$ is not calculated because it cannot be measured by the scanner. The Group of Three diffusion pattern results in an altered steady state ratio given by:

$$
\frac{\mathbf{M}(n+1)}{\mathbf{M}(n)}=\frac{\mathbf{M}(n+4)}{\mathbf{M}(n+3)} .
$$

Using this equation, we will solve for $M_{y^{\prime}}(n+1) / M_{y^{\prime}}(n)$ in terms of $T_{1}, T_{2}, \mathrm{TR}, \theta$, $\mathrm{b}$, and $\mathrm{D}$. We begin in steady state with transverse and longitudinal magnetization:

$$
\mathbf{M}(n)=\left(\begin{array}{c}
0 \\
M_{y^{\prime}} \\
M_{z}
\end{array}\right) .
$$

First we evolve the magnetization to $\mathbf{M}(n+1)$ :

$$
\mathbf{M}(n+1)=\Gamma_{\text {half }} * R_{-}(\theta) * \Gamma_{\text {half }} * \mathbf{M}(n)
$$




$$
=\left(\begin{array}{c}
0 \\
c E_{2} M_{y^{\prime}}-s \sqrt{E_{1} E_{2}} M_{z} \\
s \sqrt{E_{1} E_{2}} M_{y^{\prime}}+c E_{1} M_{z}
\end{array}\right) .
$$

Now we can solve for the left side of Equation 4.67:

$$
\frac{M_{y^{\prime}}(n+1)}{M_{y^{\prime}}(n)}=c E_{2}-s \sqrt{E_{1} E_{2}} \frac{M_{z}}{M_{y^{\prime}}} .
$$

On to the right side of Equation 4.67, beginning with $\mathbf{M}(n+3)$,

$$
\begin{gathered}
\mathbf{M}(n+3)=\Gamma_{\text {half }} * R_{-}(\theta) * \Gamma_{D} * R_{+}(\theta) * \Gamma_{\text {half }} * \mathbf{M}(n+1) \\
=\left(\begin{array}{c}
0 \\
M_{z}\left(c^{2} s\left(E_{1} E_{2} E_{D} \sqrt{E_{1} E_{2}}-E_{2}^{2} E_{D} \sqrt{E_{1} E_{2}}-E_{1}^{2} \sqrt{E_{1} E_{2}}\right)-s^{3} E_{1} E_{2} \sqrt{E_{1} E_{2}}\right) \\
M_{y^{\prime}}\left(c^{3} E_{2}^{3} E_{D}+c s^{2}\left(E_{1} E_{2}^{2} E_{D}+E_{1} E_{2}^{2}-E_{1}^{2} E_{2}\right)\right)+ \\
M_{y^{\prime}}\left(c^{2} s\left(E_{2}^{2} E_{D} \sqrt{E_{1} E_{2}}-E_{1} E_{2} \sqrt{E_{1} E_{2}}+E_{1}^{2} \sqrt{E_{1} E_{2}}\right)+s^{3} E_{1} E_{2} E_{D} \sqrt{E_{1} E_{2}}\right) \\
+M_{z}\left(c^{3} E_{1}^{3}+c s^{2}\left(E_{1}^{2} E_{2}+E_{1}^{2} E_{2} E_{D}-E_{1} E_{2}^{2} E_{D}\right)\right)
\end{array}\right) .
\end{gathered}
$$

Solving for $\mathbf{M}(n+4)$ :

$$
\mathbf{M}(n+4)=\Gamma_{\text {half }} * R_{+}(\theta) * \Gamma_{\text {half }} * \mathbf{M}(n+3)
$$




$$
=\left(\begin{array}{c}
0 \\
M_{y^{\prime}}\left(c^{4} E_{2}^{4} E_{D}+s^{4} E_{1}^{2} E_{2}^{2} E_{D}\right. \\
\left.+c^{2} s^{2}\left(E_{1}^{3} E_{2}+E_{1} E_{2}^{3}+2\left(E_{1} E_{2}^{3} E_{D}-E_{1}^{2} E_{2}^{2}\right)\right)\right) \\
+M_{z}\left(c^{3} s\left(E_{1}^{3} \sqrt{E_{1} E_{2}}-E_{1}^{2} E_{2} \sqrt{E_{1} E_{2}}-E_{2}^{3} E_{D} \sqrt{E_{1} E_{2}}-E_{1} E_{2}^{2} \sqrt{E_{1} E_{2}}-E_{1} E_{2}^{2} E_{D}^{2} E_{D} \sqrt{E_{1} E_{2}}+E_{1}^{2} E_{2} E_{D} \sqrt{E_{1} E_{2}}\right)\right. \\
M_{y^{\prime}}\left(c^{3} s\left(E_{1}^{3} \sqrt{E_{1} E_{2}}-E_{1}^{2} E_{2} \sqrt{E_{1} E_{2}}-E_{2}^{3} E_{D} \sqrt{E_{1} E_{2}}+E_{1} E_{2}^{2} E_{D} \sqrt{E_{1} E_{2}}\right)\right. \\
+c s^{3}\left(E_{1}^{2} E_{2} \sqrt{E_{1} E_{2}}-E_{1} E_{2}^{2} \sqrt{E_{1} E_{2}}-E_{1} E_{2}^{2} E_{D} \sqrt{E_{1} E_{2}}+E_{1}^{2} E_{2} E_{D} \sqrt{E_{1} E_{2}}\right) \\
+M_{z}\left(c^{4} E_{1}^{4}+s^{4} E_{1}^{2} E_{2}^{2}\right. \\
+c^{2} s^{2}\left(E_{1}^{3} E_{2} E_{D}+E_{1} E_{2}^{3} E_{D}+2\left(E_{1}^{3} E_{2}-E_{1}^{2} E_{2}^{2} E_{D}\right)\right)
\end{array}\right)
$$

By rearranging the transverse component of Equation 4.67, we have:

$$
\begin{gathered}
M_{y^{\prime}}(n+1) * M_{y^{\prime}}(n+3)=M_{y^{\prime}}(n+4) * M_{y^{\prime}}(n), \\
M_{y^{\prime}}(n+1) * M_{y^{\prime}}(n+3)-M_{y^{\prime}}(n+4) * M_{y^{\prime}}(n)=0 .
\end{gathered}
$$

We again solve for the ratio of $\frac{M_{z}}{M_{y^{\prime}}}$, and substitute that into Equation 4.71. First we 
substitute the expression for $M_{y^{\prime}}(n+4)$ :

$$
\begin{array}{r}
M_{y^{\prime}}(n+4) * M_{y^{\prime}}(n)=M_{y^{\prime}}^{2}\left(c^{4} E_{2}^{4} E_{D}+s^{4} E_{1}^{2} E_{2}^{2} E_{D}\right. \\
\left.+c^{2} s^{2}\left(E_{1}^{3} E_{2}+E_{1} E_{2}^{3}+2\left(E_{1} E_{2}^{3} E_{D}-E_{1}^{2} E_{2}^{2}\right)\right)\right) \\
+M_{y^{\prime}} M_{z} \sqrt{E_{1} E_{2}}\left(c^{3} s\left(E_{1}^{3}-E_{1}^{2} E_{2}-E_{2}^{3} E_{D}+E_{1} E_{2}^{2} E_{D}\right)\right. \\
\left.+c s^{3}\left(E_{1}^{2} E_{2}-E_{1} E_{2}^{2}-E_{1} E_{2}^{2} E_{D}+E_{1}^{2} E_{2} E_{D}\right)\right),
\end{array}
$$

and the expressions for $M_{y^{\prime}}(n+1)$ and $M_{y^{\prime}}(n+3)$ :

$$
\begin{array}{r}
M_{y^{\prime}}(n+1) * M_{y}(n+3)=M_{y^{\prime}}^{2}\left(c^{4} E_{2}^{4} E_{D}+c^{2} s^{2}\left(E_{1} E_{2}^{3} E_{D}+E_{1} E_{2}^{3}-E_{1}^{2} E_{2}^{2}\right)\right) \\
+M_{y^{\prime}} M_{z} \sqrt{E_{1} E_{2}}\left(c^{3} s\left(E_{1} E_{2}^{2} E_{D}-2 E_{2}^{3} E_{D}-E_{1}^{2} E_{2}\right)+c s^{3}\left(E_{1}^{2} E_{2}-2 E_{1} E_{2}^{2}-E_{1} E_{2}^{2} E_{D}\right)\right) \\
+M_{z}^{2}\left(s^{4} E_{1}^{2} E_{2}^{2}+c^{2} s^{2}\left(E_{1} E_{2}^{3} E_{D}+E_{1}^{3} E_{2}-E_{1}^{2} E_{2}^{2} E_{D}\right)\right) .
\end{array}
$$

We now substitute our expressions for $M_{y^{\prime}}(n+1) * M_{y}(n+3)$ and $M_{y^{\prime}}(n+4) *$ $M_{y}(n)$ into Equation 4.77:

$$
\begin{array}{r}
0=M_{z}^{2}\left(s^{4} E_{1}^{2} E_{2}^{2}+c^{2} s^{2}\left(E_{1} E_{2}^{3} E_{D}+E_{1}^{3} E_{2}-E_{1}^{2} E_{2}^{2} E_{D}\right)\right) \\
-M_{y^{\prime}} M_{z} \sqrt{E_{1} E_{2}}\left(c^{3} s\left(E_{1}^{3}+E_{2}^{3} E_{D}\right)+c s^{3}\left(E_{1}^{2} E_{2} E_{D}+E_{1} E_{2}^{2}\right)\right) \\
-M_{y^{\prime}}^{2}\left(c^{2} s^{2}\left(E_{1}^{3} E_{2}+E_{1} E_{2}^{3} E_{D}-E_{1}^{2} E_{2}^{2}\right)+s^{4} E_{1}^{2} E_{2}^{2} E_{D}\right) .
\end{array}
$$


Rearranging terms and dividing by $M_{y^{\prime}}$, we have a quadratic equation in $M_{z} / M_{y^{\prime}}$ :

$$
\begin{array}{r}
0=\left(\frac{M_{z}}{M_{y^{\prime}}}\right)^{2} s^{2} E_{1} E_{2}\left(s^{2} E_{1} E_{2}+c^{2}\left(E_{2}^{2} E_{D}+E_{1}^{2}-E_{1} E_{2} E_{D}\right)\right) \\
-\frac{M_{z}}{M_{y^{\prime}}} c s \sqrt{E_{1} E_{2}}\left(c^{2}\left(E_{1}^{3}+E_{2}^{3} E_{D}\right)+s^{2}\left(E_{1}^{2} E_{2} E_{D}+E_{1} E_{2}^{2}\right)\right) \\
\quad-s^{2} E_{1} E_{2}\left(c^{2}\left(E_{1}^{2}+E_{2}^{2} E_{D}-E_{1} E_{2}\right)+s^{2} E_{1} E_{2} E_{D}\right) .
\end{array}
$$

Solving the quadratic equation for $M_{z} / M_{y^{\prime}}$ gives:

$$
\frac{M_{z}}{M_{y^{\prime}}}=\frac{c s \sqrt{E_{1} E_{2}}\left(c^{2}\left(E_{1}^{3}+E_{2}^{3} E_{D}\right)+s^{2}\left(E_{1}^{2} E_{2} E_{D}+E_{1} E_{2}^{2}\right)\right) \pm \sqrt{X}}{2 s^{2} E_{1} E_{2}\left(s^{2} E_{1} E_{2}+c^{2}\left(E_{2}^{2} E_{D}+E_{1}^{2}-E_{1} E_{2} E_{D}\right)\right)}
$$

where

$$
\begin{gathered}
X \equiv c^{2} s^{2} E_{1} E_{2}\left(c^{2}\left(E_{1}^{3}+E_{2}^{3} E_{D}\right)+s^{2}\left(E_{1}^{2} E_{2} E_{D}+E_{1} E_{2}^{2}\right)\right)^{2} \\
+4 * s^{4} E_{1}^{2} E_{2}^{2}\left(s^{2} E_{1} E_{2}+c^{2}\left(E_{2}^{2} E_{D}+E_{1}^{2}-E_{1} E_{2} E_{D}\right)\right) *\left(c^{2}\left(E_{1}^{2}+E_{2}^{2} E_{D}-E_{1} E_{2}\right)+s^{2} E_{1} E_{2} E_{D}\right) \\
=c^{6} s^{2} E_{1} E_{2}\left(E_{1}^{3}+E_{2}^{3} E_{D}\right)^{2}+4 s^{8} E_{1}^{4} E_{2}^{4} E_{D} \\
+2 c^{4} s^{4} E_{1}^{2} E_{2}^{2}\left(E_{1}^{4}\left(E_{D}+2\right)-E_{1}^{3} E_{2}\left(1+2 E_{D}\right)-E_{1} E_{2}^{3} E_{D}\left(E_{D}+2\right)+6 E_{1}^{2} E_{2}^{2} E_{D}+E_{2}^{4} E_{D}\left(1+2 E_{D}\right)\right) \\
+c^{2} s^{6} E_{1}^{3} E_{2}^{3}\left(E_{1}^{2}\left(E_{D}^{2}+4\left(1+E_{D}\right)\right)+2 E_{1} E_{2}\left(E_{D}-2\left(1+E_{D}^{2}\right)\right)+E_{2}^{2}\left(1+4 E_{D}\left(1+E_{D}\right)\right)\right) \\
\frac{M_{z}}{M_{y^{\prime}}}=\frac{c^{3}\left(E_{1}^{3}+E_{2}^{3} E_{D}\right)+c s^{2}\left(E_{1}^{2} E_{2} E_{D}+E_{1} E_{2}^{2}\right) \pm \sqrt{X}}{2 s \sqrt{E_{1} E_{2}}\left(s^{2} E_{1} E_{2}+c^{2}\left(E_{2}^{2} E_{D}+E_{1}^{2}-E_{1} E_{2} E_{D}\right)\right)}
\end{gathered}
$$

Finally we substitute this equation for $M_{z} / M_{y^{\prime}}$ into equation 4.71 , and have an 
expression for the steady state ratio in terms of $T_{1}, T_{2}, T R, \theta, \mathrm{b}$, and D:

$$
\frac{M_{y^{\prime}}(n+1)}{M_{y^{\prime}}(n)}=c E_{2}-\frac{c^{3}\left(E_{1}^{3}+E_{2}^{3} E_{D}\right)+c s^{2}\left(E_{1}^{2} E_{2} E_{D}+E_{1} E_{2}^{2}\right) \pm \sqrt{X}}{2\left(s^{2} E_{1} E_{2}+c^{2}\left(E_{2}^{2} E_{D}+E_{1}^{2}-E_{1} E_{2} E_{D}\right)\right)}
$$

Equation 4.86 has a very complicated dependence on parameters which make it difficult to determine how the ratio will depend on diffusion. The (-) expression is plotted in red versus $b * D$ in Figure 4.21 with blue points representing the simulation of the magnetization evolution using the Group of Three diffusion-weighted SSFP pulse sequence, and the incoherent ratio $e^{-b D}$ is the black line.

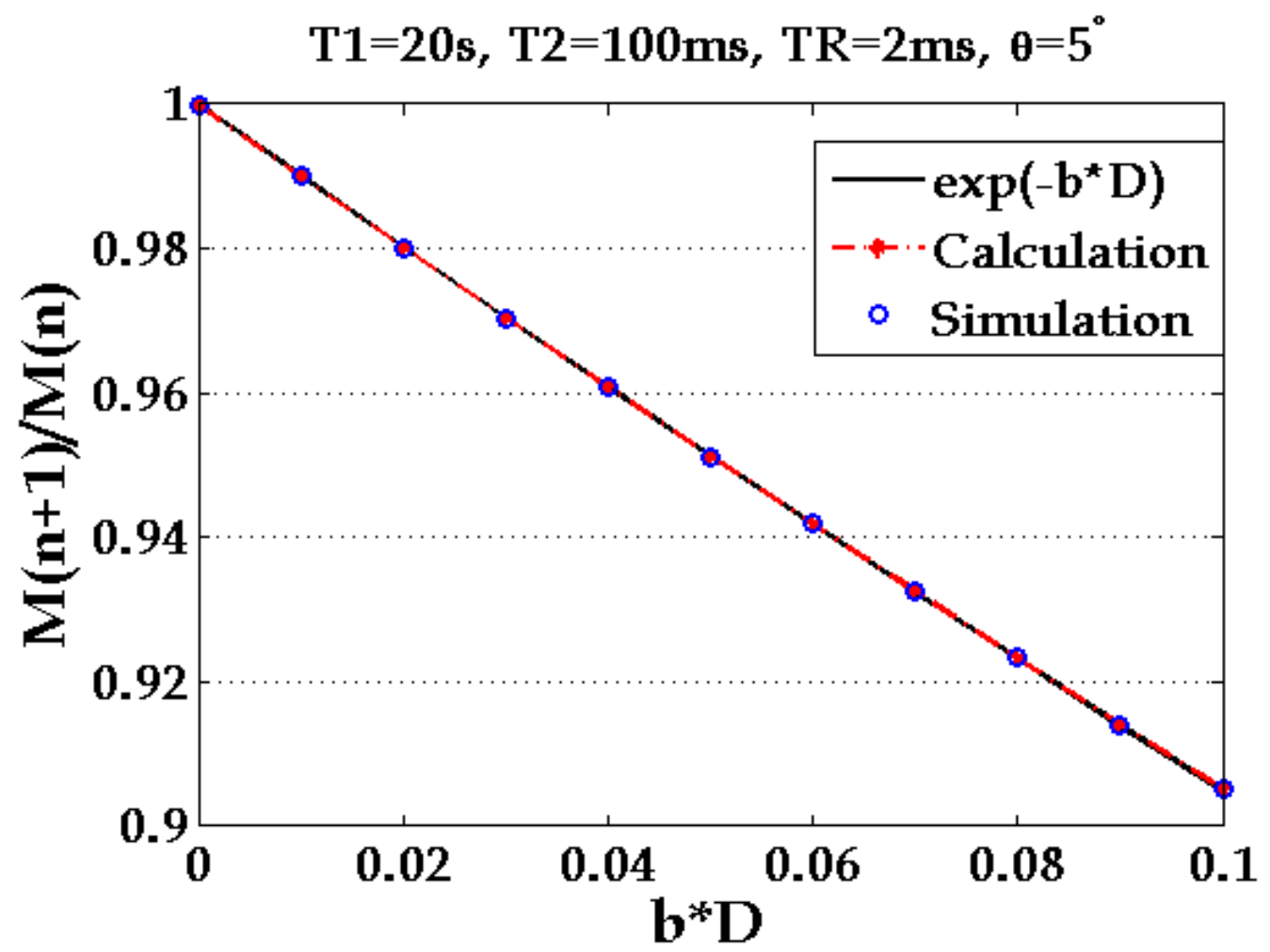

Figure 4.21: Plot of signal ratio resulting from the simulation of Group of Three diffusion SSFP (blue points), $e^{-b D}$ (black line), and Equation 4.86 (red line) versus $\mathrm{b}^{*} \mathrm{D}$. Simulation and calculation parameters were: $T R=2 \mathrm{~ms} T 1=20 \mathrm{~s}, T 2=$ $100 \mathrm{~ms}, \theta=5^{\circ}$.

Despite the complicated dependence on system parameters reflected in Equa- 
tion 4.86 , the signal ratio appears to be dominated by the diffusion attenuation in the regime of relaxation values $T_{1}$ and $T_{2}$ common in hyperpolarized helium-3 MR in the lungs. If the expression was not dominated by diffusion attenuation, it would be difficult to calculate an ADC value from the measured signal ratio given by Equation 4.86, because $T_{1}, T_{2}$, and $\theta$ must be known. However for realistic values of these parameters, the ratio expression can be approximated as:

$$
\frac{M_{y^{\prime}}(n+1)}{M_{y^{\prime}}(n)}=E_{D}=e^{-b * D},
$$

which should allow us to obtain quantitative diffusion measurements from the Group of Three diffusion-weighted SSFP pulse sequence for on-resonant magnetization.

The next step in evaluating the Group of Three method is to determine how the ratio $M_{y^{\prime}}(n+1) / M_{y^{\prime}}(n)$ changes in the presence of off-resonant magnetization. Figure 4.22 shows the results of the magnetization simulation under the Group of Three diffusion-weighted SSFP pulse sequence with off-resonant magnetization. The magnetization behavior is simulated for a particular value of diffusion attenuation, $b * D=0.05$. In Figure 4.22a, one can see there is a transition point at $\beta=60^{\circ}$. Magnetization vectors which undergo an off-resonance precession of $\beta \leq \pm 60^{\circ}$ per TR have steady state signal ratios that are less than one. For offresonance precession angles of $\beta> \pm 60^{\circ}$ per TR, the steady-state signal ratio is greater than one, a reversal of the expected contrast.

In Figure $4.22 \mathrm{~b}$, one can see that for a uniformly distributed collection of offresonance precession angles $|\beta|<70^{\circ}$ per TR, the simulated ratio is close to or less than the calculated value. For a larger spread of off-resonance precession angles, the ratio measured will be greater than the diffusion attenuation, resulting in an underestimation of the diffusion coefficient. 


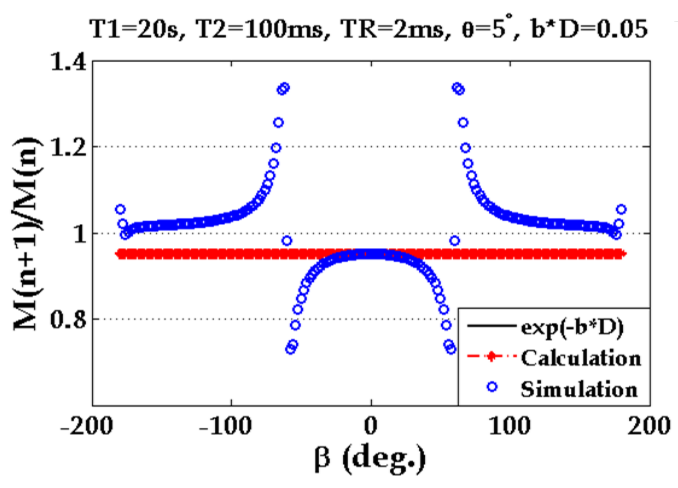

(a) Frequency Offset

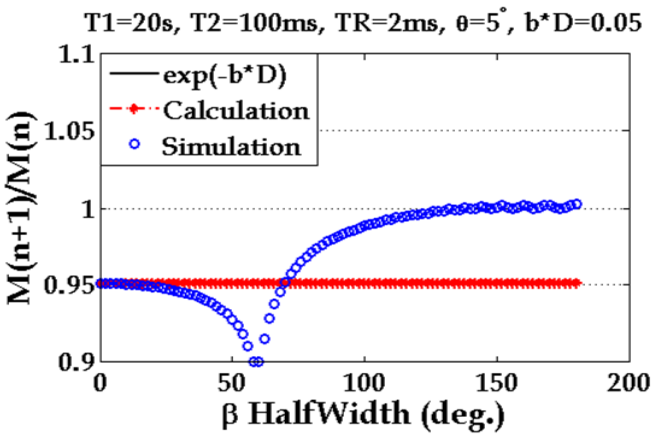

(b) Frequency Spread

Figure 4.22: Plot of signal ratio resulting from the simulation of Group of Three diffusion SSFP (blue points), diffusion attenuation (black line), and Equation 4.86 (red line) versus off resonance width, in Hz. $T_{1}=20 \mathrm{~s}, T_{2}=100 \mathrm{~ms}, \theta=5^{\circ}$, $\mathrm{TR}=2 \mathrm{~ms}$. (a) shows the effect of a single $\beta$, (b) shows effect of averaging over a collection of $\beta \mathrm{s}$.

Because the data acquisition window and the diffusion-sensitizing gradients are contained in different TR periods in the Group of Three diffusion-weighted SSFP pulse sequence, this pulse sequences permits shorter values of TR to be achieved than any of the other three variations considered here. Therefore it is possible that for short enough diffusion times, the range of off-resonant precession angles present in human lungs will be low enough to allow accurate ADC measurements to be made. We tested the Group of Three technique in phantoms and human subjects and the results are presented in Chapter 5 and Chapter 6.

\subsubsection{Before/After Diffusion-Weighted SSFP}

The final method we developed to combine SSFP with diffusion sensitization involves applying diffusion gradients in every TR window, similar to the Symmetric diffusion-weighted SSFP pulse sequence. In the Before/After diffusion-weighted pulse sequence, however, the transverse signal is measured either before or after the gradient is applied, depending on the TR period as shown in Figure 4.23. In 


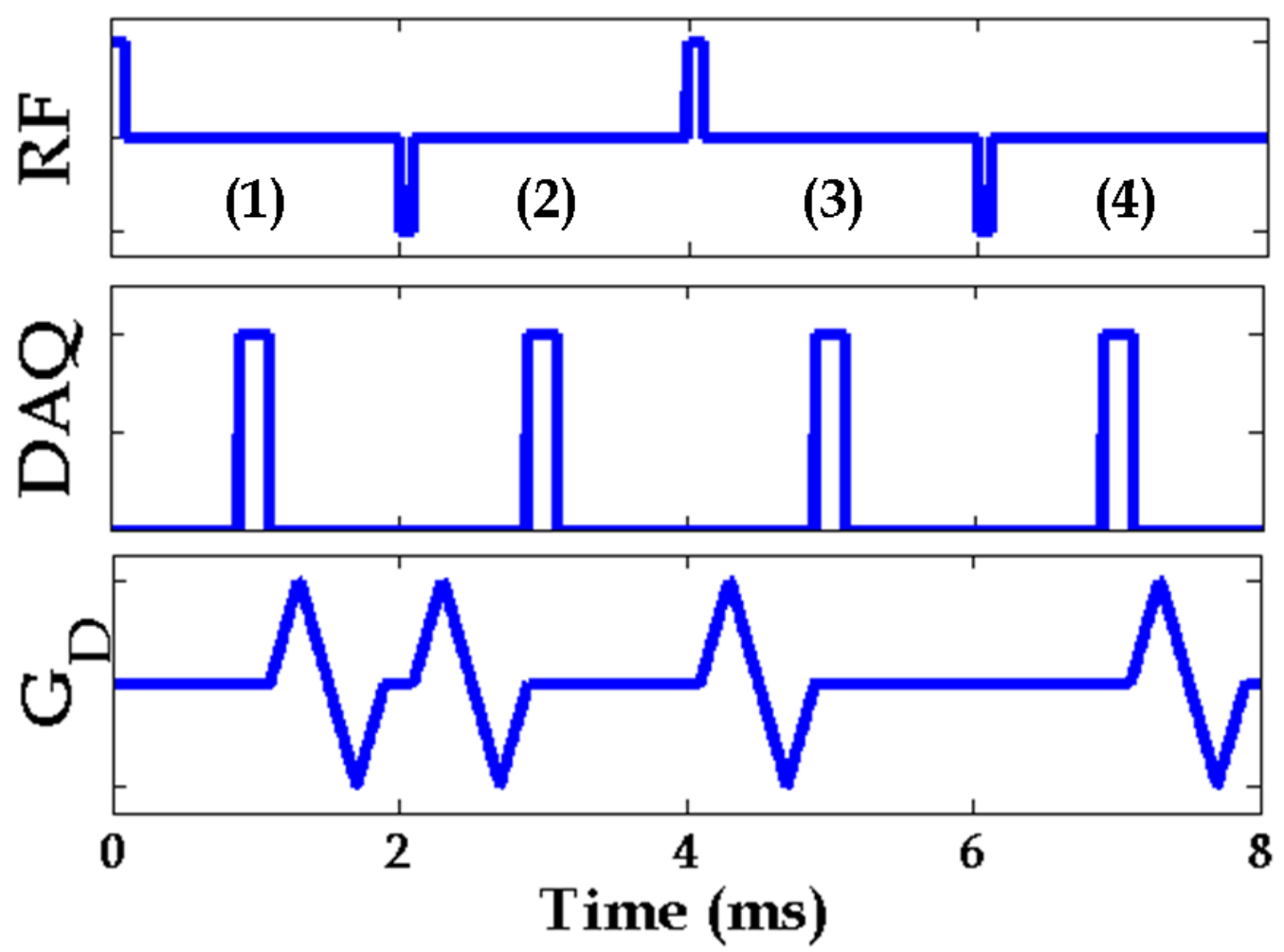

Figure 4.23: Before/After diffusion-weighted SSFP pulse sequence diagram. The diffusion gradients $\left(G_{D}\right)$ are applied either before (2 and 3 ) or after (1 and 4$)$ the centered DAQ. By alternating the order of the gradients after-before-before-after and taking the average ratio of $S$ (before)/S(after), this method normalizes for signal decline and baseline RF asymmetry.

order to avoid sensitivity to relaxation, the signal is measured at the same time $(t=T R / 2)$ during every TR period. To accommodate both of these features the diffusion gradients are placed in either the first half or the second half of the TR period, depending on the desired ordering. The minimum repetition time for the Before/After method must therefore accommodate two bipolar diffusion gradients, the data acquisition window, and the RF excitation pulse, which is much larger than required for the Group of Three pulse sequence presented in the previous section.

The steady state ratio of $M_{y^{\prime}}(n+1) / M_{y^{\prime}}(n)$ is purely determined by the diffu- 
sion attenuation,

$$
\frac{M(\text { before })}{M(\text { after })}=e^{-b * D}
$$

as the transverse relaxation is the same for both signals. As in the Symmetric diffusion pulse sequence, the is no alternating between different signal levels in the Before/After diffusion-weighted SSFP pulse sequence. There is the same amount of signal attenuation in every TR period. We theorized that the constant decay combined with the measurement of the signal at $t=T R / 2$ would eliminate sensitivity of the steady state ratio to off-resonant magnetization. We explored the behavior of the magnetization under the Before/After diffusion-weighted pulse sequence using our magnetization simulation. The results of the simulation are plotted in Figure 4.24 for on-resonant magnetization.

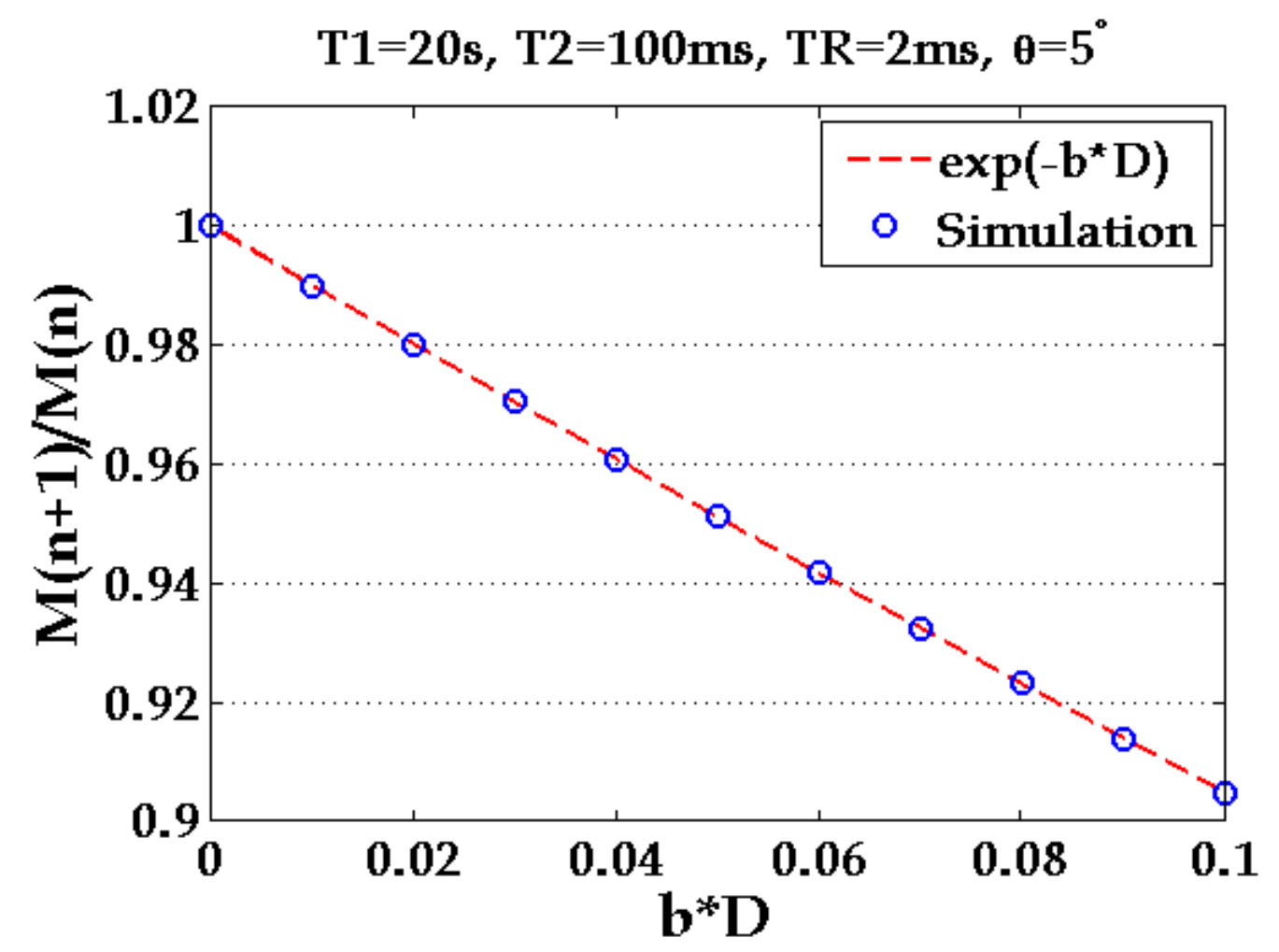

Figure 4.24: Plot of signal ratio resulting from the simulation of Before/After diffusion-weighted SSFP (blue points) and diffusion attenuation (red line) versus $b^{*}$ D. $T 1=20 s, T 2=100 \mathrm{~ms}, \theta=5^{\circ}$. 
As expected, the Before/After diffusion-weighted SSFP pulse sequence produces a signal ratio equal to the diffusion attenuation in the absence of off-resonance magnetization. We incorporated off-resonant magnetization into the simulation as before, and plotted the signal ratio in Figure 4.25.

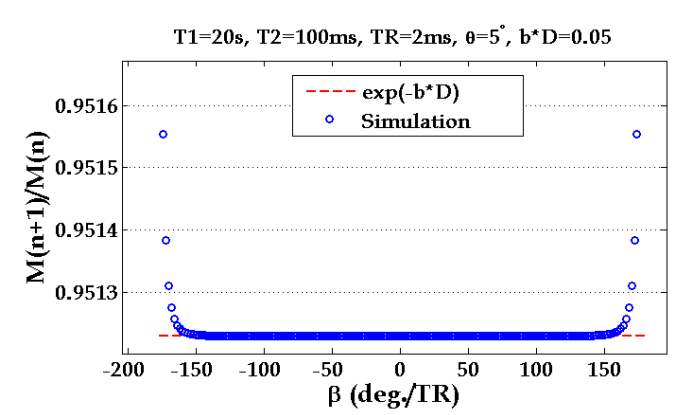

(a) Frequency Offset

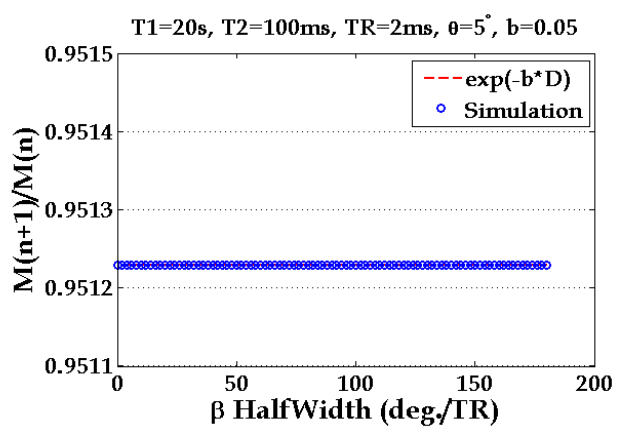

(b) Frequency Spread

Figure 4.25: Plot of signal ratio resulting from the simulation of Before/After diffusion-weighted SSFP (blue points) and diffusion attenuation (red line) versus off-resonant precession angle, in degrees/TR. $T_{1}=20 \mathrm{~s}, T_{2}=100 \mathrm{~ms}, \theta=5^{\circ}$, $\mathrm{TR}=2 m s, b * D=0.05$. (a) shows the effect of a single $\beta$, (b) shows effect of averaging over a collection of $\beta$ values.

The ratio of $M$ (before) $/ M($ after $)$ is robust against off-resonant precession, a clear benefit of the central sampling method compared to the Symmetric diffusion pulse sequence. This effect is illustrated by looking at the signal size at $t=T R / 2$ as a function of off-resonance angle $\beta$ in Figure 4.26. The magnitude of the signal at $t=T R / 2$ goes down as $\beta$ increases, so off-resonant spins do not contribute as much to the value when averaging spins together. 


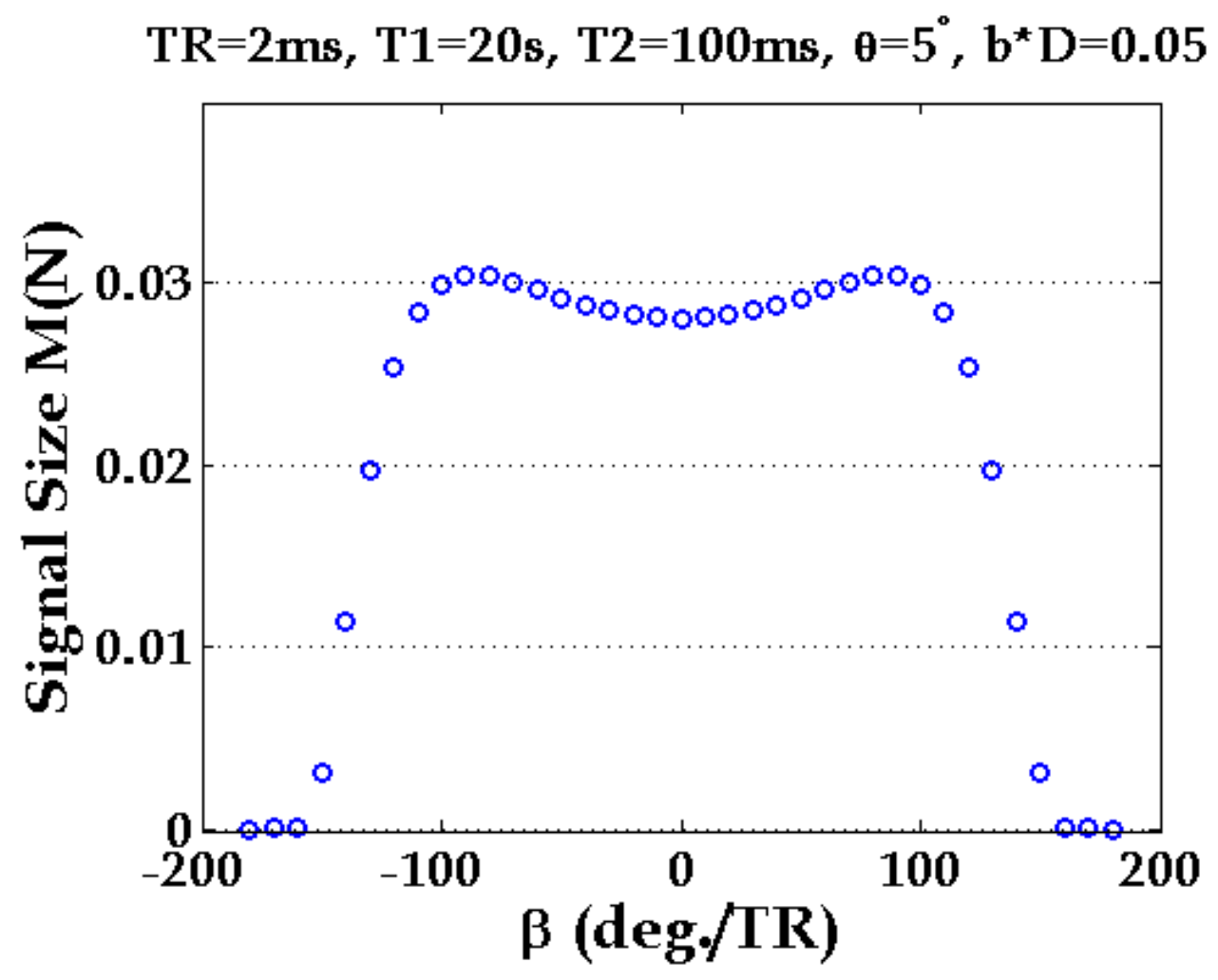

Figure 4.26: Plot of transverse magnetization magnitude at resulting from simulation of Before/After diffusion-weighted SSFP (blue points) vs off-resonance precession angle $\beta$. 


\subsection{Conclusions}

In examining the effect of an SSFP pulse sequence on hyperpolarized magnetization, we have shown that in the absence of diffusion attenuation, the magnetization reaches a steady state in which the ratio of consecutive signals approaches a constant value which depends on relaxation time constants $T_{1}$ and $T_{2}$ and pulse sequence parameters $T R$ and $\theta$. For a given combination of these parameters, the ratio of transverse magnetization in consecutive TR periods, $M_{y^{\prime}}(n+1) / M_{y^{\prime}}(n)$, is much closer to unity in coherent SSFP pulse sequences than in incoherent pulse sequences, due to the recycling of the previous transverse magnetization. This smaller signal decline per RF excitation leads to the ability to use larger flip angles, which results in higher SNR than is possible using an incoherent pulse sequence.

The increased SNR achievable using SSFP pulse sequences lead us to develop a diffusion-weighted application of SSFP for hyperpolarized gases at short diffusion times. The high diffusivity of helium-3 means that measurable diffusion attenuation can be imparted by a bipolar pair of diffusion gradients executed in a single TR period. We investigated several methods of incorporating diffusion sensitization into an SSFP pulse sequence while maintaining a short diffusion time.

Each of the methods we investigated produced well-behaved diffusion-weighted contrast in the presence of strictly on-resonant magnetization. In the case of the Symmetric and the Before/After diffusion-weighted SSFP pulse sequences, the ADC value was straight forward to calculate from the steady state signal ratio $M_{y^{\prime}}(n+1) / M_{y^{\prime}}(n)$. By contrast, the Asymmetric and the Group of Three diffusionweighted SSFP pulse sequences produced very different steady state magnetization behaviors in which the signal attenuation was not the same after each pulse, leading to complex signal ratio expressions. The complicated dependence of the ratio $M_{y^{\prime}}(n+1) / M_{y^{\prime}}(n)$ on parameters other than $D$ makes quantization of diffu- 
sion difficult in the Asymmetric diffusion-weighted SSFP pulse sequence, whereas simplification is possible for the Group of Three version for the values of the relaxation time constants $T_{1}$ and $T_{2}$ present when using hyperpolarized helium-3 MR in the lung.

The simulations of magnetization behavior in the presence of off-resonant magnetization helped further clarify the strengths and weaknesses of each of the pulse sequences we developed for SSFP diffusion weighting. Only the Before/After diffusion-weighted pulse sequence creates a steady state ratio that is robust against the presence of off-resonant magnetization. Each of the other diffusion-weighted SSFP pulse sequences creates a steady state ratio which deviates from the calculated value for off-resonant spins.

In our investigation of the non-diffusion weighted SSFP pulse sequence, we discovered a previously unreported baseline asymmetry between transverse signals measured at alternating RF phase angles. The presence of a baseline asymmetry would compromise our ability to make diffusion measurements from steady state signal ratios, so we studied the effect and developed a technique to reduce our sensitivity to it, which will be discussed in Chapter 5 . 


\section{Chapter 5}

\section{SSFP Measurements of}

\section{Time-Dependent Diffusion}

Our exploration of diffusion-weighted steady state free precession (SSFP) for hyperpolarized gases in the previous chapter produced several methods of incorporating diffusion-sensitizing gradients into an SSFP pulse sequence. The Asymmetric diffusion-weighted SSFP pulse sequence provides a great enhancement of contrast between diffusion-weighted and non-diffusion-weighted acquisitions when compared to incoherent diffusion-weighted pulse sequences, but the degree of contrast is highly sensitive to the presence of off-resonant magnetization. The Group of Three diffusion-weighted SSFP pulse sequence also produces results that are sensitive to off-resonant magnetization, but it has the shortest minimum TR of our candidate diffusion-weighted SSFP pulse sequences and may therefore not have significant off-resonant phase accumulation. The Before/After diffusionweighted SSFP pulse sequence was developed to be insensitive to the presence of off-resonant magnetization for the purposes of calculating an ADC.

In this chapter, we use the SSFP pulse sequences developed in Chapter 4 to make diffusion measurements. As described in Chapter 2, the diffusion coefficient 
$D$ depends on the measurement time in the presence of restriction, $D=D(t)$. To test the ability of diffusion-weighted SSFP pulse sequences to produce accurate diffusion measurements, we measured the time-dependent diffusion coefficient of helium-3 in spherical glass phantoms. We developed versions of the Group of Three and Before/After diffusion-weighted SSFP pulse sequences that measured the ADC at multiple short diffusion times in a single scan. The phantoms were completely filled with glass beads of either $1 \mathrm{~mm}, 2 \mathrm{~mm}$, or $3 \mathrm{~mm}$ diameter. For densely packed spherical beads, the theoretical behavior of the time-dependent diffusivity is known, which allowed us to assess the accuracy of our ADC measurements at short diffusion times. We then tested the multi-diffusion time SSFP pulse sequence in human volunteers, to observe the time dependence of diffusivity in the lung and to better understand the range of diffusivities we should expect to observe in diffusion-weighted SSFP imaging measurements made in the short time-scale regime.

\subsection{Time Dependence of the Restricted Diffusion Co- efficient}

Mitra, et al have shown that for very short diffusion times $t$, the time-dependent diffusivity $D(t)$ depends linearly on the square-root of the diffusion time, with a slope proportional to the surface-to-volume ratio $(S / V)$ of the space available for diffusion [30],

$$
\begin{gathered}
D(t)=D_{0}\left[1-\frac{4}{9 \sqrt{\pi}} \frac{S}{V} \sqrt{D_{0} t}\right] \\
\text { for } t<<\frac{1}{(S / V)^{2} D_{0}} \equiv t_{0}
\end{gathered}
$$


where $t_{0}$ represents a transition diffusion time between the short time-scale linear region, and the non-linear region. The theoretical value of $S / V$ for a dense pack of spherical beads of diameter $d$ is given by [34]:

$$
\frac{S}{V}=\frac{6(1-\phi)}{d \phi}
$$

The parameter $\phi$ is the porosity of the space, defined as the fraction of empty space in the volume available for diffusion. In our bead phantoms, the diffusing space consists of the relatively large gaps or pores between the beads, which are connected to each other by very narrow pathways along the edges of the beads. For a random dense pack of identical spheres, the theoretical minimum porosity is $\phi=0.39$ [34], independent of sphere diameter. The larger beads create larger pores, but there is a higher number of pores in a phantom filled with smaller beads.

In order to extract an $S / V$ ratio from time-dependent ADC data, multiple measurements must be made in the linear region governed by Equation 5.2 to perform a linear fit and calculate a slope. We calculated $t_{0}$ for each bead phantom to give an idea of the short time-scale region extent. We do not expect the diffusion coefficient to vary linearly all the way up to $t_{0}$, but it is unclear from Equation 5.2 how far below $t_{0}$ the linear behavior ceases. The calculated values of $t_{0}$ are presented in Table 5.1 for both pure helium-3 and helium-3 dilute in air. For comparison, $t_{0}$ is also estimated for healthy human lungs, with $S / V=250 \mathrm{~cm}^{-1}$ [74]. Since the motivation for the short time-scale diffusion measurements is for in vivo applications in the lung, we only consider gas mixtures at atmospheric pressure, in contrast to previous applications $[35,36]$.

As we will see later, the minimum diffusion time at which we were able generate measurable diffusion attenuation using our diffusion-weighted SSFP pulse sequence was $\Delta \sim 300 \mu \mathrm{s}$. For the $1 \mathrm{~mm}$ and $2 \mathrm{~mm}$-bead phantoms, the calculated 


\begin{tabular}{|c|c|c|c|}
\hline Gas & $D_{0}$ & Object & $t_{0}$ \\
\hline \multirow{3}{*}{ Pure ${ }^{3} \mathrm{He}$} & \multirow{3}{*}{$1.9 \mathrm{~cm}^{2} / \mathrm{s}$} & $3 \mathrm{~mm}$ Beads & $540 \mu \mathrm{s}$ \\
& & $2 \mathrm{~mm}$ Beads & $240 \mu \mathrm{s}$ \\
& & $3 \mathrm{~mm}$ Beads & $60 \mu \mathrm{s}$ \\
\hline & & $2 \mathrm{~mm}$ Beads & $1160 \mu \mathrm{s}$ \\
${ }^{3} \mathrm{He}$ Dilute in air & \multirow{2}{*}{$0.88 \mathrm{~cm}^{2} / \mathrm{s}$} & $510 \mu \mathrm{s}$ \\
& & 1mm Beads & $130 \mu \mathrm{s}$ \\
& & Healthy Alveoli & $20 \mu \mathrm{s}$ \\
\hline
\end{tabular}

Table 5.1: Short time-scale limits for helium-3 in bead phantoms and healthy human lungs.

values of $t_{0}$ are below $300 \mu$ s for pure helium-3 diffusion, indicating that we would not be able to make measurements in the linear region to calculate an $S / V$ ratio. In the case of diluted helium-3, we may be able to access the linear regime in the $2 \mathrm{~mm}$ and $3 \mathrm{~mm}$-bead phantoms, but that ability greatly depends on how much less than $t_{0}$ the linear region of diffusion time is. We are interested in verifying the accuracy of ADC measurements made with a diffusion-weighted SSFP pulse sequence by comparing experimental results to theoretical time-dependent diffusivity, and the calculations in Table 5.1 indicate that it is unlikely that we will be able to use the linear short time-scale region to do so with helium-3 at atmospheric pressure. In addition, experimental measurements of helium-3 in human lungs will be even further from the linear regime than in the bead phantoms.

At very long diffusion times, the diffusion coefficient in a system of well-connected pores, such as exists in the bead phantoms, approaches a constant value that is known as the tortuosity limit [32]:

$$
\begin{gathered}
D(\Delta)=\frac{D_{0}}{\alpha}, \\
\text { for } \Delta>\frac{L^{2}}{D_{0}} .
\end{gathered}
$$


The constant $\alpha$ quantifies the tortuosity of the space, a geometric factor describing the crookedness of the diffusion path, i.e., how much longer the path is than the direct length from starting position to ending position. At long diffusion times in a well-connected restrictive space, the diffusivity can be thought of as the free diffusion coefficient reduced by the path-increase factor $\alpha$ [75]. In a pack of spherical beads, $\alpha=1 / \sqrt{\phi}[34]$, which is once again independent of sphere diameter. The diffusion time at which Equation 5.4 becomes valid is related to the free diffusivity $D_{0}$, and a diffusion length $L$ that is some fraction of the pore space. In the case of spherical beads, Mair et al observed $L=(0.3 \rightarrow 1.0) * d$ for various bead diameters $d$ [34]. When the diffusion time reaches values described by Equation 5.5, the diffusivity is insensitive to the pore size of the surrounding environment. Therefore, long time-scale diffusion is not of great interest when trying to distinguish between samples with similar connectivity but varying pore size.

In the region between the short time-scale linear behavior and the long timescale tortuosity limit, the so-called Padé approximation can be used to model the behavior of the diffusion coefficient [76, 77]. The Padé approximation is an interpolation between the known short time-scale and long time-scale behavior:

$$
\frac{D(t)}{D_{0}}=1-(1-\sqrt{\phi}) * \frac{\frac{4}{9 \sqrt{\pi}} \frac{S}{V} \sqrt{D_{0} t}+(1-\sqrt{\phi}) \frac{D_{0} t}{D_{0} \theta}}{(1-\sqrt{\phi})+\frac{4}{9 \sqrt{\pi}} \frac{S}{V} \sqrt{D_{0} t}+(1-\sqrt{\phi}) \frac{D_{0} t}{D_{0} \theta}} .
$$

The Padé equation (Equation 5.6) contains a parameter $\theta$, which has the unit of time and represents the point at which the time dependence of the diffusivity transitions between the short and long time-scale regimes. The $\theta$ parameter will be different for different structural environments, such as bead sizes. Instead of using 


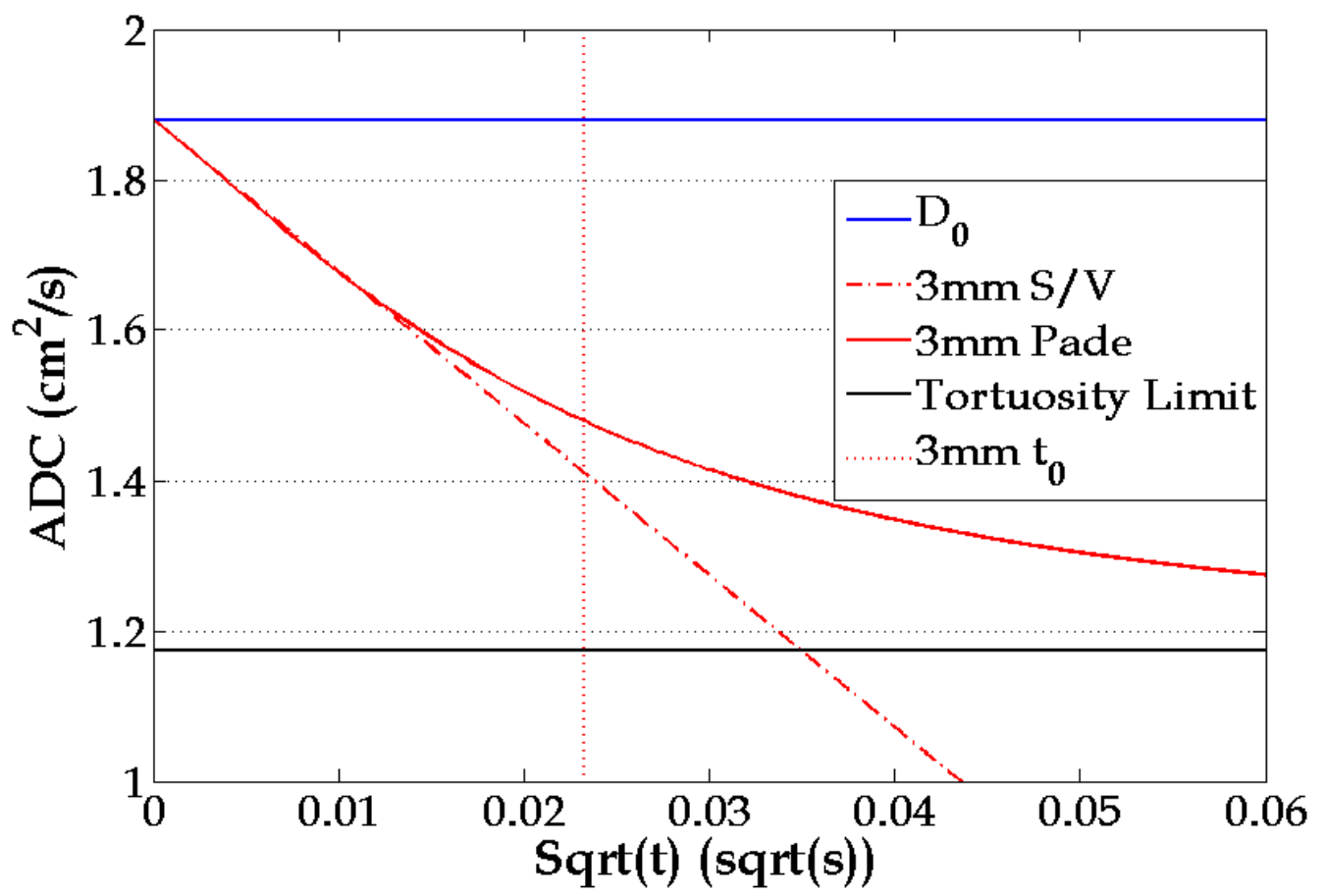

Figure 5.1: The time dependence of the diffusion coefficient of helium-3 under restricted diffusion. The blue line represents the free diffusion coefficient $D_{0}$ and the black line is the long time-scale tortuosity limit, $\sqrt{\phi} * D_{0}$. The dashed red line is the short time-scale linear dependence. The red solid line is the Padé equation, which results from an interpolation between the short and the long time-scale regions. The vertical dotted red line is the square root of the diffusion time $t_{0}$. The linear behavior of the diffusivity breaks down well before the calculated $\sqrt{t_{0}}$.

$\theta$ as a fit parameter, the use of the unitless Padé length,

$$
L_{p}=\frac{\sqrt{D_{0} \theta}}{d}
$$

separates out the dependence on bead diameter $d$. The Padé length was originally given as $L_{p}=0.145$ by Latour et al [76] for water-infused glass beads using an estimation for the tortuosity limit. It was later calculated by Mair et al, using measured short time-scale and long time-scale xenon-infused glass bead data, to be $L_{p}=0.132$ [35]. The graph of the Padé equation in Figure 5.1 shows that the time-dependent diffusivity curve deviates from the linear region at approximately 
$\frac{1}{2} \sqrt{t_{0}}$. Therefore, in order to calculate a surface-to-volume ratio, one would need to measure the ADC at several points below $\frac{t_{0}}{4}$ to perform a linear fit. Our experiment to verify the accuracy of ADC values calculated from a diffusion-weighted SSFP pulse sequence was designed to make diffusion measurements at the shortest practical diffusion times, which fall in the transition region between the short and the long time-scales for helium-3 at atmospheric pressure. Therefore we used the Padé equation to interpret our measurements of time-dependent diffusion.

\subsection{General Experimental Methods}

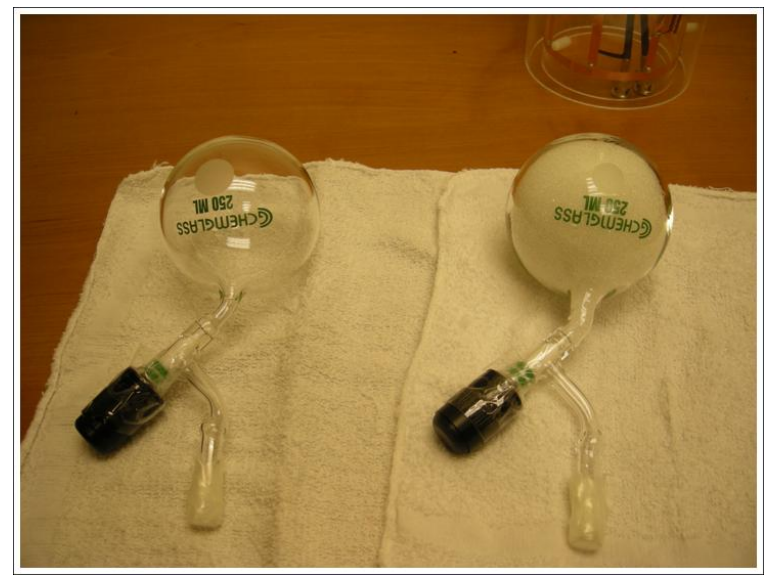

Figure 5.2: Spherical glass phantoms used for global ADC measurements of restricted diffusion. The empty cell on the left is a free-diffusion phantom, and the phantom on the right is filled with $1 \mathrm{~mm}$ diameter beads. Additional phantoms with $2 \mathrm{~mm}$ and $3 \mathrm{~mm}$ beads were also used.

We performed pulse sequence tests and made global ADC measurements in four spherical glass phantoms. The phantoms used for these experiments consisted of four $100 \mathrm{~mL}$ glass spheres, three of which were filled with glass beads of diameters 1, 2, or $3 \mathrm{~mm}$ (Figure 5.2). The empty phantom provided measurements of the free diffusion coefficient of the gas, while the bead-filled phantoms provided measurements of restricted diffusion, similar to lung tissue. Before each 
measurement session, helium-3 was polarized with the Hybrid Helium Polarizer (Chapter 3) to $\sim 60 \%$. The phantom to be used was connected to a $250 \mathrm{~mL}$ Tedlar® plastic bag which served as a gas reservoir, as shown in Figure 5.3. The reservoir was used to provide a supply of helium-3 at atmospheric pressure that could be drawn into the glass phantom. This arrangement ensured that the gas pressure inside the phantom was atmospheric at the time of measurement, and also permitted the hyperpolarized helium-3 to be kept in the relaxation-friendly environment of the Tedlar $\AA$ bag during transport to the scanner and positioning in the coil.

Both the phantom and bag were first evacuated through the gas-handling system of the hybrid polarizer, and the phantom valve was closed. The reservoir bag was then filled with a volume of ${ }^{3} \mathrm{He}$, slightly larger than the empty volume of the phantom cell $\left(V_{\mathrm{He} 3} \sim 40 \mathrm{~mL}\right.$ for the bead-filled phantoms and $V_{\mathrm{He}} \sim 120 \mathrm{~mL}$ for the free-diffusion phantom) measured by a $1.5 \mathrm{~L}$ syringe. The rig was placed in the 1.5 T MR scanner (Avanto, Siemens Medical Solutions, Malvern PA) with a homebuilt RF coil tuned to the helium-3 resonance. The phantom was positioned in the coil and immediately before data collection, the reservoir valve and the phantom valve were opened to draw ${ }^{3} \mathrm{He}$ from the reservoir bag into the phantom.

The resonant frequency of the gas in the phantom was determined using the frequency adjustment tool on the MR scanner. This tool applies an excitation RF pulse at a user-specified frequency and amplitude, and then displays the resulting free-induction decay (FID) as well as its Fourier transform, which shows the frequency profile of the measured signal. The applied voltage of the RF excitation was set to the minimum value $V=0.1 \mathrm{~V}$, resulting in a very low flip angle excitation. The peak signal was recorded, and the system frequency was set to the frequency of the peak. After the calibration was performed, the pulse sequence was applied. Data were analyzed using Matlab software (MathWorks, Natick MA). 


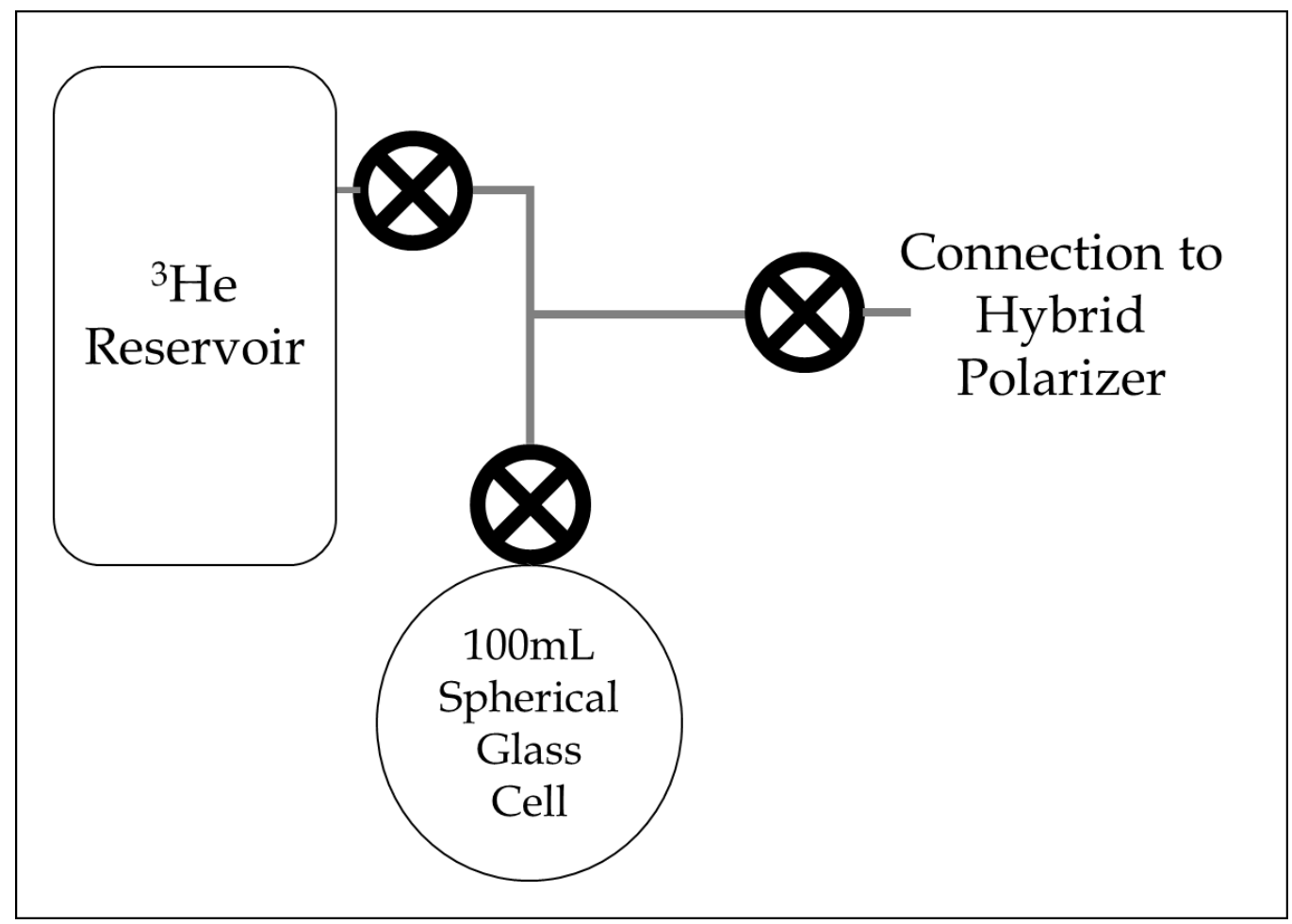

Figure 5.3: Schematic of setup used to dispense polarized helium-3 to each phantom.

\subsection{Bead-Phantom Relaxation Measurements}

When designing the diffusion-weighted SSFP pulse sequences which were used to make ADC measurements in our bead phantoms, we had to consider the environment the magnetization would experience, specifically $T_{1}$ and $T_{2}^{*}$. In contrast to the human hyperpolarized gas $\mathrm{MR}$, there was no breath-hold timing requirement when working with phantoms. However, there was still the requirement that the measurement must be completed before the longitudinal signal decayed to zero. We wanted to apply the exact same pulse sequence in each of our phantoms, so the total acquisition time of the sequence was therefore limited by the phantom with the shortest $T_{1}$. Furthermore, in order for the pulse sequence to be coherent and take advantage of the recycled transverse magnetization, $T R$ must be shorter than $T_{2}^{*}$. The repetition time of our diffusion-weighted SSFP pulse sequence was 
therefore limited by the phantom with the shortest $T_{2}^{*}$.

We measured the longitudinal relaxation $T_{1}$ in our bead phantoms using the frequency-adjustment tool on the scanner, by recording the peak signal from an excitation RF pulse applied every five seconds. The expected signal relationship between consecutive points is

$$
S(n)=S(n-1) e^{-\frac{t}{T_{1}}} \cos \theta,
$$

where $S(n)$ is the peak signal after the n-th excitation, $t$ is the time between excitations, and $\theta$ is the applied flip angle. We used the minimum voltage in the frequency-adjustment tool $V=0.1 \mathrm{~V}$ to minimize the flip angle and ensure that $\cos \theta \sim 1$. The signal measurements were normalized to the first of the series, then the natural logs of the data were fit to a line. The slope of this line was the inverse of the time constant. The results of the relaxation measurements are presented in Figure 5.4a. The shortest values of $T_{1}$ were measured in the $1 \mathrm{~mm}$ and $2 \mathrm{~mm}$ bead phantoms, $T_{1}(1 \mathrm{~mm})=11.9 \mathrm{~s}$ and $T_{1}(2 \mathrm{~mm})=11.4 \mathrm{~s}$.

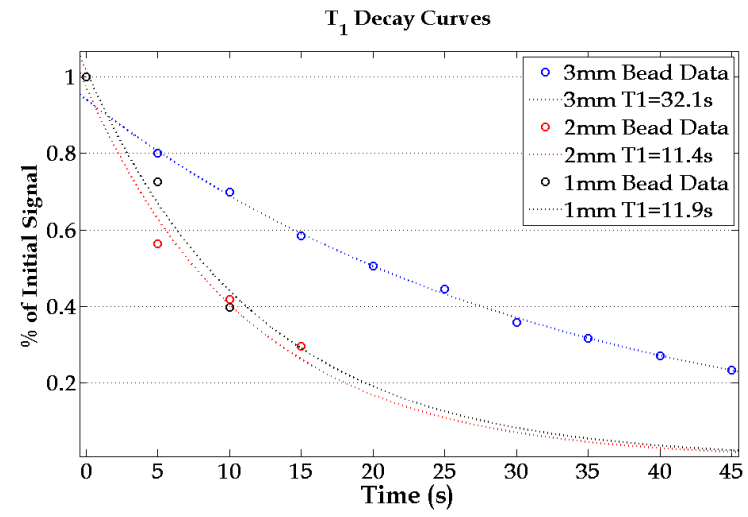

(a) Global bead $T_{1}$.

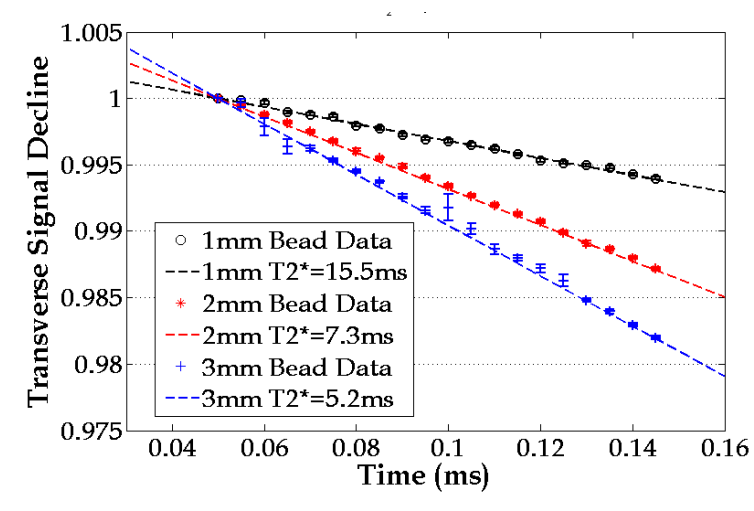

(b) Global bead $T_{2}^{*}$.

Figure 5.4: Measurements of longitudinal and transverse magnetization decay were made in each phantom. (a) Longitudinal decay was measure by applying an excitation RF pulse at a constant interval and recording the peak signal size. (b) Transverse magnization relaxation was measured by applying an excitation pulse and fitting the decay measured during the acquisition window. 
The transverse relaxation was determined by fitting the signal decline during a data acquisition window. We applied a simple SSFP pulse sequence which consisted of slice-selective RF excitations and data acquisition periods repeated several hundred times. The signal was sampled every $5 \mu \mathrm{s}$ for the duration of the DAQ window, in this case $200 \mu \mathrm{s}$. The expected signal decline is:

$$
S(t)=S_{0} e^{-\frac{t}{T_{2}^{*}}},
$$

where $S_{0}$ is the initial signal. A region of 20 samples was selected, and the signal at each point was normalized to the first point in the selected region. The normalized signal values were averaged over all of the RF excitations collected in the scan.

The standard error in the mean of all of the excitations gave a measure of statistical error, represented by the error bars on the points on Figure 5.4b. The statistical error was an additional input to the linear fit of the natural log of the normalized signal measurements. The shortest value of $T_{2}^{*}$ was measured in the $3 \mathrm{~mm}$ bead phantom, $T_{2}^{*}(3 \mathrm{~mm})=5.2 \mathrm{~ms}$. We used these relaxation measurements to guide the design of our SSFP pulse sequences, in particular having a $T R<5 \mathrm{~ms}$, and a total acquisition times $T_{a c q} \leq 10 \mathrm{~s}$.

\subsection{Pulse Sequence Tests}

We tested three diffusion-weighted SSFP pulse sequence variations using our phantoms. First, we tested the Asymmetric SSFP diffusion-weighted pulse sequence shown in Figure 4.16 in the free-diffusion phantom, expecting to measure a larger difference between diffusion-weighted and non-diffusion-weighted acquisitions than $e^{-b * D}$. Our simulations in Chapter 4 showed that the Asymmetric SSFP diffusion-weighted pulse sequence only produced high contrast for on-resonance 
magnetization. The free-diffusion phantom contained the fewest air/glass interfaces of our phantoms, and was expected to have the smallest background magnetic field gradients due to susceptibility changes and therefore the least off-resonant magnetization. The diffusion gradients were applied both before and after the DAQ window, which was centered at $t=T R / 2$. We made diffusion-weighted measurements at a single diffusion time, $\Delta=400 \mu \mathrm{s}$, and the amplitude of the diffusion gradients was varied from $G_{D}=5-20 \mathrm{mT} / \mathrm{m}$. The ratio of consecutive pulses was calculated for each RF excitation, then averaged over the number of repetitions in each scan. The results of this test are plotted in Figure 5.5 versus the b-value, calculated from the applied diffusion gradients according to the trapezoidal b-value equation listed in Table 2.4.

The average signal ratio is far less than the steady state ratio $M(n+1) / M(n)$ predicted by equation 4.60. We see that even in our optimal environment with respect to off-resonant magnetization, the enhanced contrast potential in the Asymmetric diffusion-weighted SSFP pulse sequence is not realized. Due to the lack of contrast enhancement in this test, and the inability to extract an ADC from Asymmetric diffusion-weighted SSFP measurements, we eliminated Asymmetric diffusion as a candidate pulse sequence.

In Section 4.5, we discussed a baseline difference between signal levels measured after RF excitations depending on the direction, $\pm \theta$, or RF phase setting $0 / 180^{\circ}$. When acquiring data with either the Group of Three diffusion-weighted SSFP pulse sequence or the Before/After diffusion-weighted SSFP pulse sequence, we were able to employ two tactics to minimize the effect of the baseline signal differences. First, we set our RF phase to the zero-crossing angles, measured on the $1.5 \mathrm{~T}$ scanner to be $\sim 100^{\circ} / 280^{\circ}$, to minimize the size of the effect. However since we do not know the origin of the asymmetry, we do not know how it might 


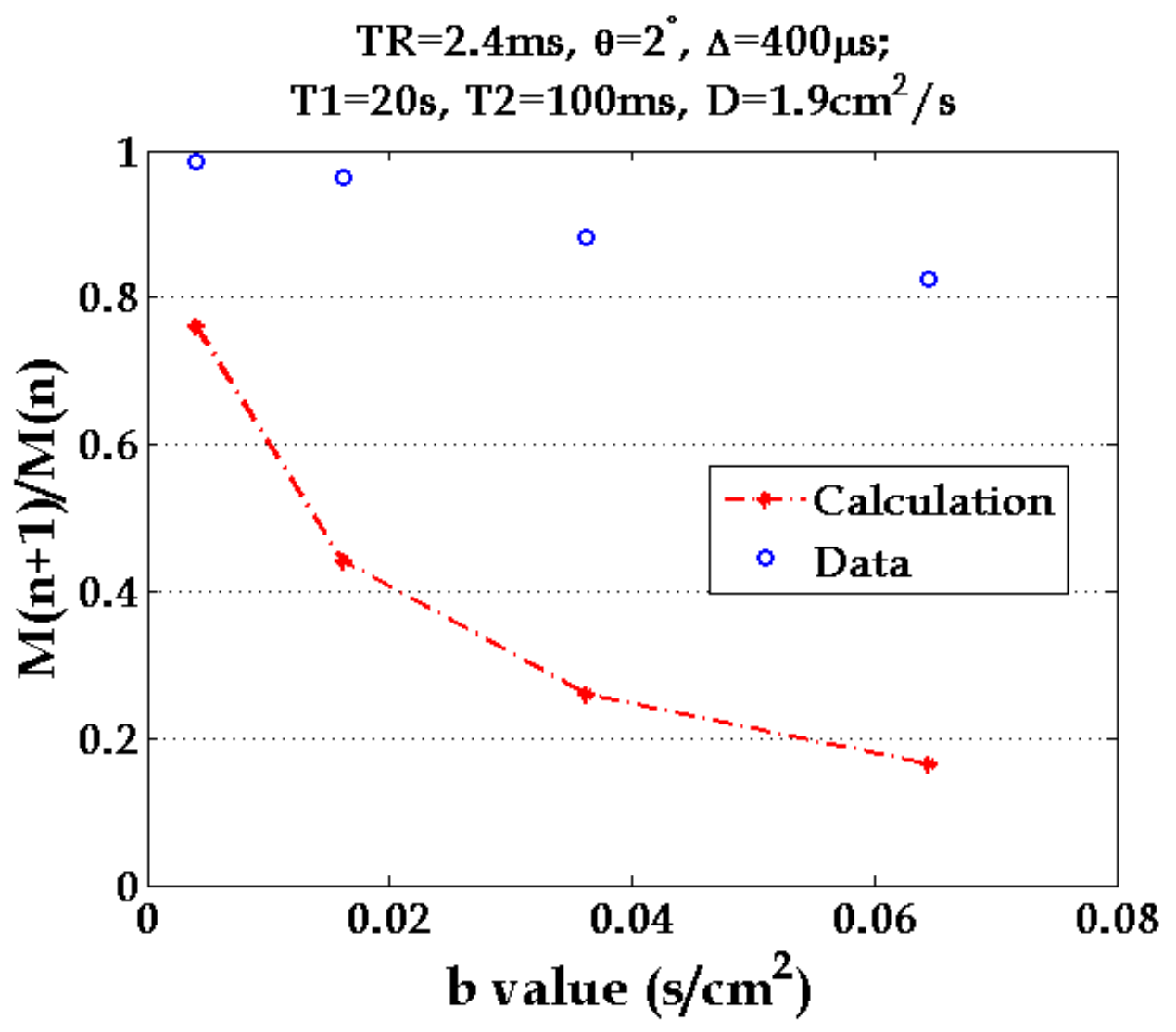

Figure 5.5: Plot of results (blue points) and equation 4.60 (red line) versus b. TR = $2.4 \mathrm{~ms}, \theta=2^{\circ}, \Delta=400 \mu \mathrm{s}$, RF Width $=400 \mu \mathrm{s}$. For the calculation: $T_{1}=20 \mathrm{~s}$, $\mathrm{T}_{2}=100 \mathrm{~ms}$, and $D=1.9 \mathrm{~cm}^{2} / \mathrm{s}$.

change with hardware, time, etc. So additionally, we acquired data with diffusionweighting in each combination of the RF phase before taking ratios to calculate diffusion attenuation $\left(\frac{S\left(100^{\circ}\right)+S\left(280^{\circ}\right)}{S\left(280^{\circ}\right)+S\left(100^{\circ}\right)}\right)$ to cancel out any residual difference. This strategy leads to a doubling of our acquisition time for our measurements.

We made time-dependent (multiple- $\Delta$ ) diffusion measurements of ${ }^{3} \mathrm{He}$ at atmospheric pressure in two of the spherical glass phantoms, one with free diffusion and one with diffusion restricted by $3 \mathrm{~mm}$-diameter glass beads using the Group of Three diffusion-weighted SSFP pulse sequence. The global measurement was made using a slice-selective RF excitation pulse, which served to reduce the extent of the measurement to a centered $20 \mathrm{~mm}$ section of the phantoms to avoid 
exciting any freely diffusing helium-3 present in the stem of the phantoms. The simulations in Chapter 4 indicate that the steady-state signal ratio is sensitive to the presence of off-resonant magnetization. However, because the Group of Three diffusion-weighted SSFP pulse sequence separates the TR periods with diffusionsensitizing gradients from TR periods with data acquisition windows, we were able to use a very short repetition time, $T R=1.76 \mathrm{~ms}$. We were optimistic that the off-resonant magnetization would not have time to accumulate much phase in our selected slice, and therefore would not significantly distort the signal ratio. The scan consisted of many excitations at each diffusion time $\Delta$, which were averaged together. Using the Group of Three diffusion-weighted SSFP pulse sequence shown in Figure 4.20, the ratio of $S(2) / S(1)$ had the opposite baseline bias as the ratio $S(5) / S(4)$. These values were averaged to eliminate the RF phase baseline: $\frac{S(2)+S(5)}{S(1)+S(4)}$. Each DAQ window consisted of 40 samples acquired during the TR period. On a sample-by-sample basis, the $S(2)$ and $S(5)$ excitations were averaged, and the corresponding $S(1)$ and $S(4)$ excitations were combined. The signal ratio was calculated from these values and averaged over the number of repetitions performed at each value of $\Delta$. Lastly, the ratio was averaged over the data acquisition window samples, with the standard error in the mean of the DAQ window samples providing a measure of statistical error. The ADC value was calculated from the ratio as follows,

$$
A D C(\Delta)=-\frac{\log R(\Delta)}{b(\Delta)}
$$

where $b(\Delta)$ was calculated from the applied diffusion gradients. We expected to measure $D_{0} \approx 1.9 \mathrm{~cm}^{2} / \mathrm{s}$ in the empty phantom, and values between $D_{0}$ and the tortuosity limit $\sqrt{\phi} * D_{0}=0.63 D_{0}$ in the $3 \mathrm{~mm}$-bead phantom. The results of this test are plotted in Figure 5.6.

In the empty phantom, we measured $A D C=1.902 \pm 0.005 \mathrm{~cm}^{2} / \mathrm{s}$, similar to 


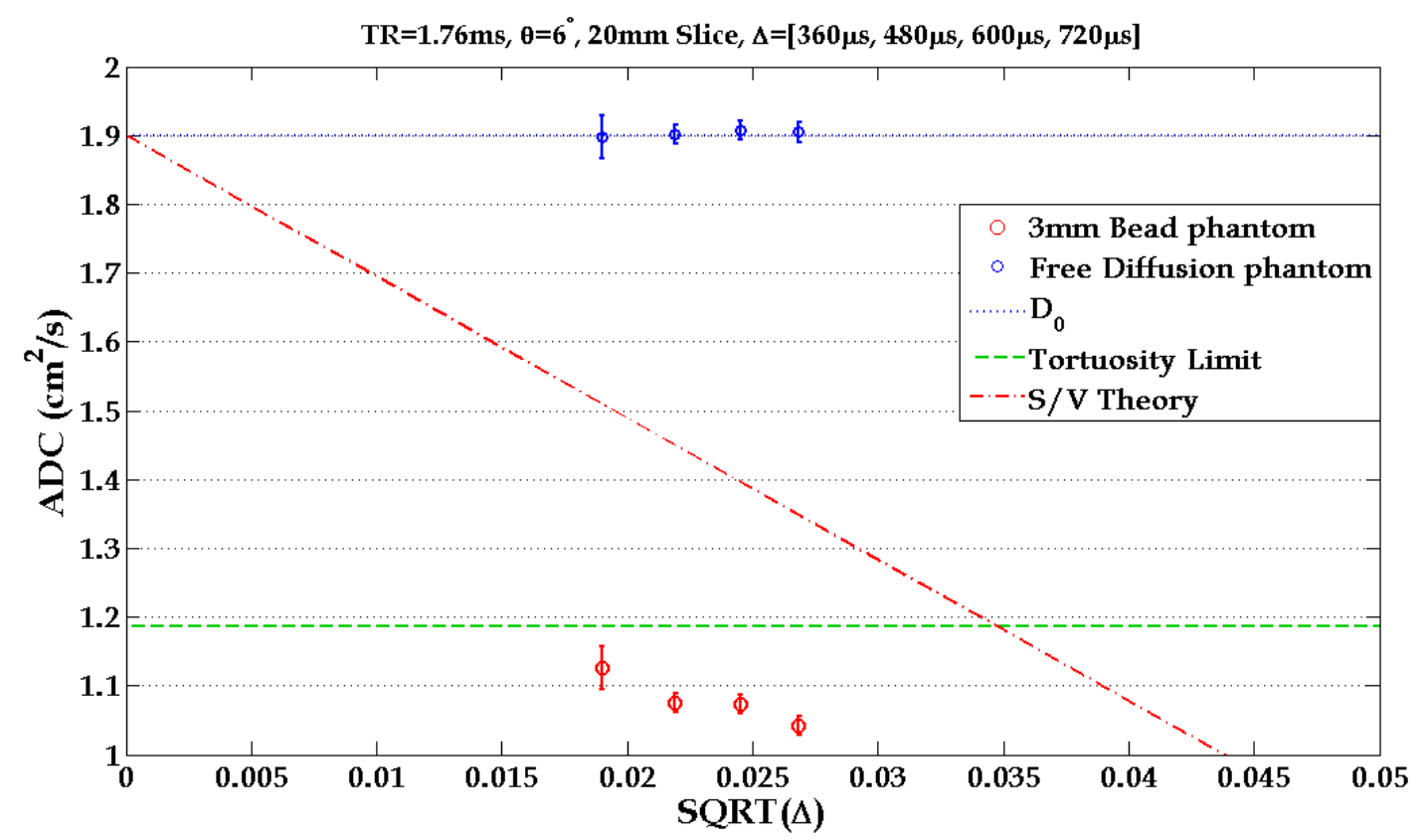

Figure 5.6: Plot of Free Diffusion Phantom Data (blue points) and 3mm-Bead Phantom Data (red points); the theoretical boundaries $D_{0}$ (blue dots) and tortuosity limit (green dashes); and the short $\Delta$ time dependence (red dashed line). $\mathrm{TR}=1.76 \mathrm{~ms}, \theta=6^{\circ}, \Delta=360,480,600,720 \mu \mathrm{s}$.

the previously measured self-diffusion coefficient of helium-3 [78]. In the $3 \mathrm{~mm}$ bead phantom however, the measured ADC was lower than the calculated tortuosity limit. These results might be explained by significant off-resonant phase accumulation during the TR window in the $3 \mathrm{~mm}$-bead phantom even for the short repetition time used, $T R=1.76 \mathrm{~ms}$. The resulting ADC values are well below the expected values, which our simulation from Section 4.6.3 indicates is consistent with off-resonant precession angles greater than $\beta=60^{\circ} / T R$. Based on this test, we used the Before/After diffusion-weighted SSFP pulse sequence for the remainder of our time-dependent diffusion measurements, due to its theoretical ability to produce a steady state signal ratio that is independent of off-resonance precession angle $\beta$, as suggested by our magnetization simulation results in Chapter 4 .

The Before/After pulse sequence shown in Figure 4.23, in which the gradient 
timing is alternated: (1) after the DAQ window, (2) before the DAQ window, (3) before the DAQ window, (4) after the DAQ window, is able to correct for baseline offset due to RF phase asymmetry. The two sets of acquisitions produced by this pulse sequence are averaged to produce the steady state ratio:

$$
R=\frac{S_{A v g}(\text { Before })}{S_{A v g}(\text { After })}=\frac{S(2)+S(3)}{S(1)+S(4)}
$$

where $S(1)$ is the signal measured during a TR period with diffusion gradient timing (1) described above, $S(2)$, etc. We repeated the test performed with the Group of Three diffusion-weighted SSFP pulse sequence in the free diffusion phantom and the $3 \mathrm{~mm}$-bead phantom using the Before/After diffusion-weighted SSFP pulse sequence, and the results of this test are presented in Figure 5.7. We measured similar values in the free-diffusion phantom with the Before/After pulse sequence as we had with the Group of Three pulse sequence. However, we were able to measure plausible values for the $3 \mathrm{~mm}$-bead phantom ADC, despite having a longer repetition time $(T R=3.64 \mathrm{~ms})$ than used in the Group of Three SSFP diffusion pulse sequence. The ADC values measured in the $3 \mathrm{~mm}$-bead phantom lay above the theoretical $S / V$ line, indicating that these measurements were made in the transition region governed by the Padé approximation. 


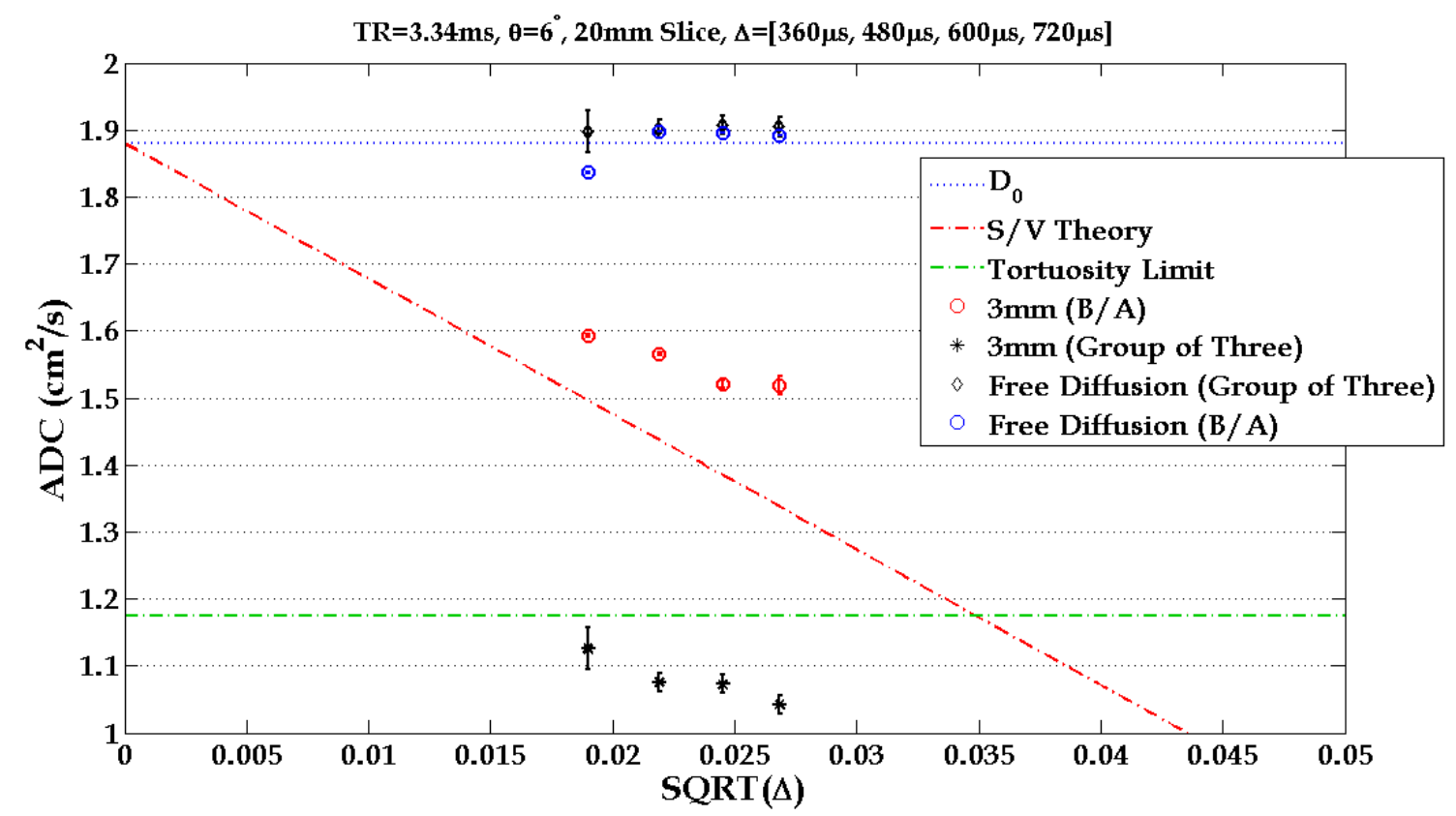

Figure 5.7: Plot of Free-Diffusion Phantom Data (blue points) and $3 \mathrm{~mm}$-bead Phantom Data (red points) obtained with the Before/After diffusion-weighted SSFP pulse sequence; the theoretical boundaries $D_{0}$ (blue dots) and tortuosity limit (green dashes); and the short $\Delta$ time dependence (red dashed line). TR $=3.64 \mathrm{~ms}$, $\theta=6^{\circ}, \Delta=360,480,600,720 \mu \mathrm{s}$. Also plotted are the data points acquired using the Group of Three pulse sequence in the free diffusion phantom (black diamonds) which agree with the values measured by Before/After, and in the $3 \mathrm{~mm}$ bead phantom (black stars), which lie significantly below the theoretically possible range. 


\subsection{Systematic Effects in Diffusion-weighted SSFP}

Under the influence of an SSFP pulse sequence, the magnetization evolution takes some time to reach its steady-state signal level. Since quantitative ADC measurements require magnetization evolution to be in steady state, we needed to allow for this transition in our pulse sequence design. The magnitude of the steady state signal level can be different for different degrees of signal attenuation during the TR period, such as would be produced by changing the diffusion gradient amplitude or $\Delta$ value. In an effort to study the magnetization evolution due to diffusion attenuation at multiple values of $\Delta$, we designed a pulse sequence in which we gradually changed the diffusion gradient amplitude at a particular value of $\Delta$, then changed the diffusion time and repeated the process. The basic unit of four TR periods in the Before/After diffusion-weighted SSFP pulse sequence has alternating diffusion gradient timing: (1) after the DAQ window, (2) before the DAQ window, (3) before the DAQ window, and (4) after the DAQ window. This unit of four RF excitations was repeated twenty times at a particular value of diffusion gradient amplitude, and then the amplitude was incrementally changed. The amplitude series used in this test is shown in Figure 5.8a, and the diffusion times of the gradients are shown in Figure 5.8b. We included diffusion gradients which consisted of a positive lobe followed by a negative lobe (referred to as positive-polarity gradients) and diffusion gradients which consisted of a negative lobe followed by a positive lobe (negative-polarity gradients) in our amplitude test. The gradual change in diffusion gradient amplitude was expected to reduce the number of pulses required to get the magnetization evolution into the steady-state level.

We tested this pulse sequence in the both the free-diffusion phantom and the $3 \mathrm{~mm}$-bead phantom using hyperpolarized helium-3. The raw data acquired from the amplitude-ramp test in the free-diffusion phantom are presented in Figure 5.9a, 
where each point is the average of the signal over the DAQ window, and the different colors correspond to the (1) after, (2) before, (3) before, (4) after cycling of diffusion gradient applications. As the diffusion gradient amplitude increases (Figure 5.8a), the signal amplitude decreases for data acquired when a diffusion gradient has been applied before the DAQ (2 and 3) and increases for data acquired when the diffusion gradient is applied after the DAQ (1 and 4). The larger $\Delta$ value applied in the second half of the acquisition (Figure 5.8b) results in a larger separation between before and after points. Figure $5.9 \mathrm{~b}$ shows a small section of the data in Figure 5.9a, in which the raw signal diverges during the transition period and then approaches steady state at each amplitude step, even though the changes in amplitude are small. Our attempt to keep the transverse magnetization in steady state did not succeed. The ratio of consecutive signals during the transition period does not reflect the steady state characteristics of the system. Therefore only points after the transition region were averaged to calculate the ADC value.

Additionally, we noted that there is a measurable difference between the ratio measured when the bipolar diffusion gradient has a positive polarity compared to when the gradient has a negative polarity. In Figure 5.10, the steady-state ratios from signals when the diffusion gradients had a positive lobe first are averaged separately from acquisitions with negative diffusion gradient lobe first. The ratio is again calculated by:

$$
R=\frac{S(2)+S(3)}{S(1)+S(4)}
$$

on a sample by sample basis throughout the DAQ window. The repetitions at each amplitude where averaged once the signal level had reached steady state, and then the data were averaged across the DAQ samples. The standard error in the mean of the DAQ samples was used as a measure of statistical error, represented by the error bars on the points in Figure 5.10. The average ratio is plotted versus the 
b-value of the applied diffusion gradient. The average ratio increases for both polarities with increasing $b$-value, however there is a systematic difference between the positive polarity ratio (blue crosses) and the negative polarity ratio (red circles). This analysis indicates that the Before/After diffusion-weighted SSFP pulse sequence is sensitive to systematic effects due to gradient polarity. A common technique for dealing with such effects is to acquire data in both polarity configurations and average the results. We adopted this approach in our pulse sequence design.

Figure 5.11a shows the signal ratio versus b-value curves measured in the $3 \mathrm{~mm}$ bead phantom and the free-diffusion phantom, after averaging across gradient polarity and DAQ samples. We expect the signal decline to be exponential, although it can be close to linear for low diffusion attenuation $(b * D \leq 0.1)$. Figure $5.11 \mathrm{~b}$ shows the corresponding ADC value calculated from the natural log of each data point in Figure 5.11a divided by the b-value at that point, as well as the slope of a linear fit to the natural log of all of the data. In both methods of calculating the ADC, the point generated by having the gradient amplitude set to zero was not included in the calculation. Looking at the data from the $3 \mathrm{~mm}$-bead phantom (blue points), the ADC values calculated by the individual points are centered about the value obtained by fitting the data to a line (green line). When we calculated an ADC from the free-diffusion phantom data, the values from each amplitude pointby-point (red points) are systematically lower than when we fit all of the data to a line (black line). In looking at the ratios in Figure 5.11a, the free diffusion phantom exhibits a systematic offset at the lower diffusion gradient amplitudes, resulting in a ratio greater than one (and thus a negative ADC value) for the lowest non-zero gradient amplitude. Comparing the ADC values obtained in the free-diffusion phantom to previously measured values for helium-3 self diffusion $[78,79]$, we 
believe that the calculated slope of the free-diffusion phantom data is a reliable measurement of the free diffusion coefficient, even in the presence of the systematic offset. In the $3 \mathrm{~mm}$-bead phantom, the offset has not been observed and both methods yield the same measurement of the restricted diffusion coefficient.

The results from these tests lead us to redesign our diffusion gradient amplitude course for tests in the bead phantoms. Instead of slowly increasing the amplitude of the gradient to gently transition between different steady states, we simply measured the attenuation at a large gradient amplitude and repeated the measurement several times to ensure that the system reached steady state. By reducing the number of amplitude steps in our measurement, we were able to make ADC measurements at a large number of finely-spaced diffusion times while still keeping our total acquisition time below the longitudinal relaxation time constant in the smallest beads ( $\left.T_{a c q} \leq 10 \mathrm{~s}\right)$. In order to make accurate measurements in the free diffusion phantom, we continued to use the amplitude ramp and calculated an ADC value from the slope, but this design resulted in fewer values of $\Delta$ measured over the same acquisition time. 


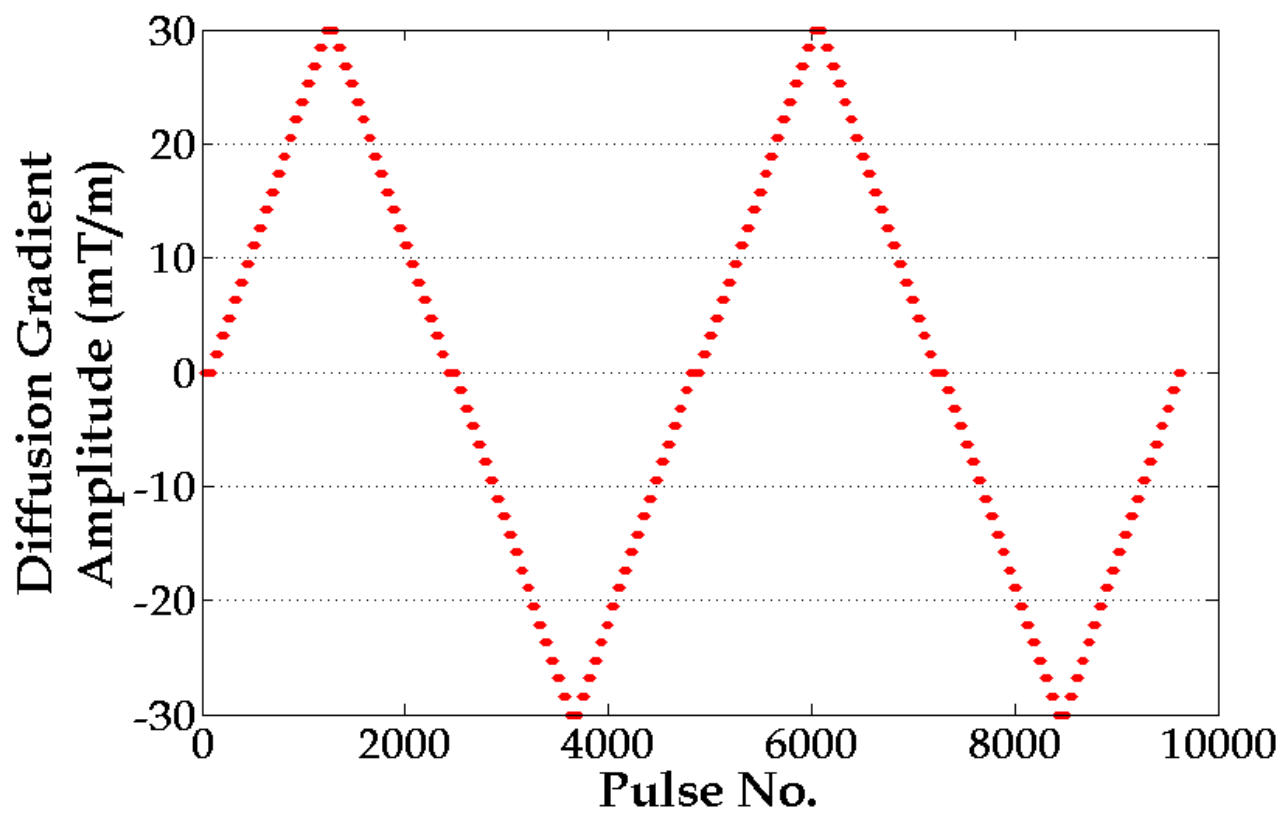

(a) Amplitude v RF Pulse

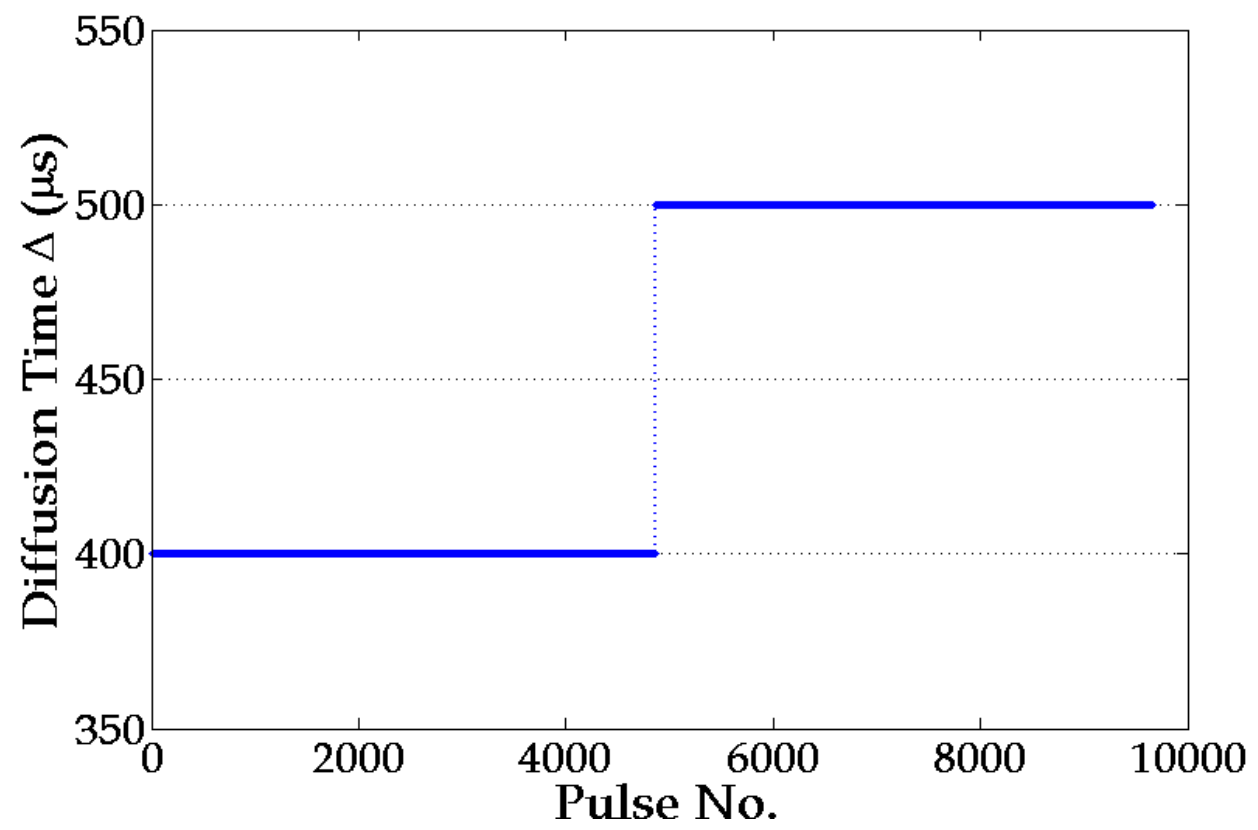

(b) $\Delta$ v RF Pulse

Figure 5.8: Amplitude ramp used in time-dependent ADC measurements designed to transition between different steady-state levels gradually. The diffusion time is increased between the second and third peaks. 


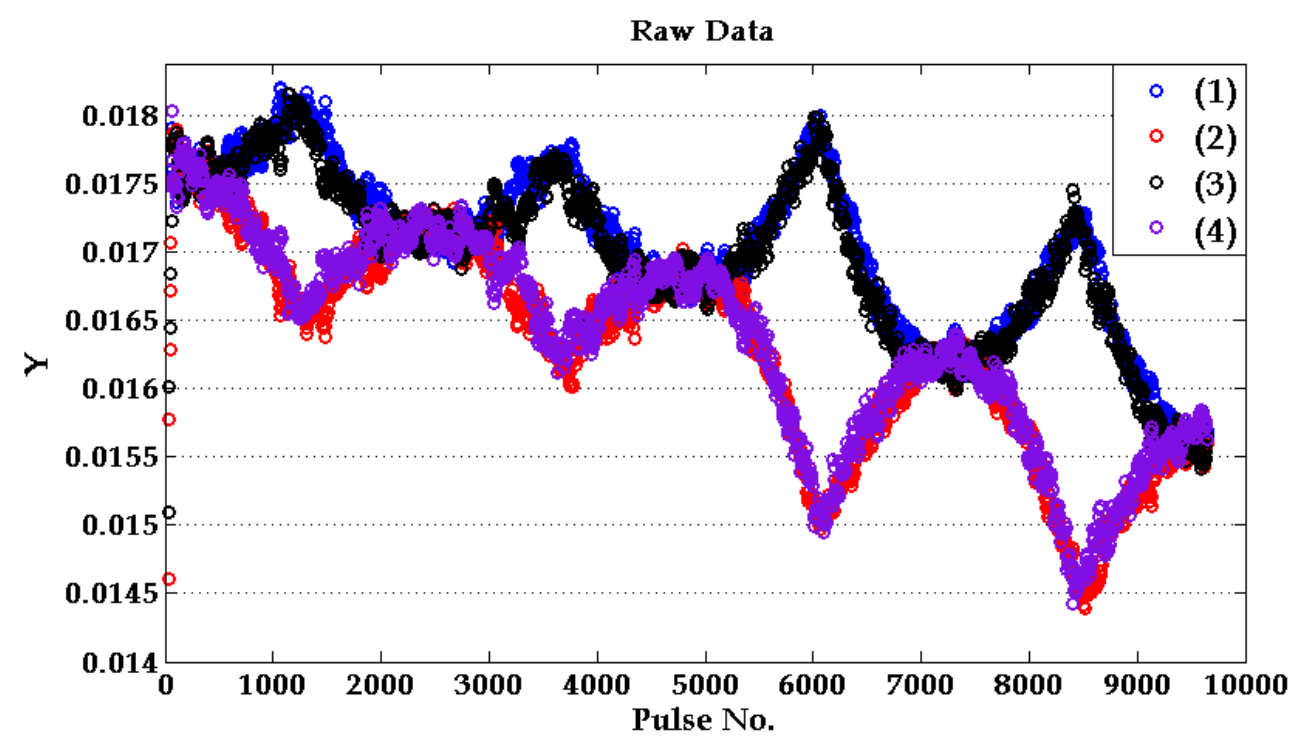

(a)

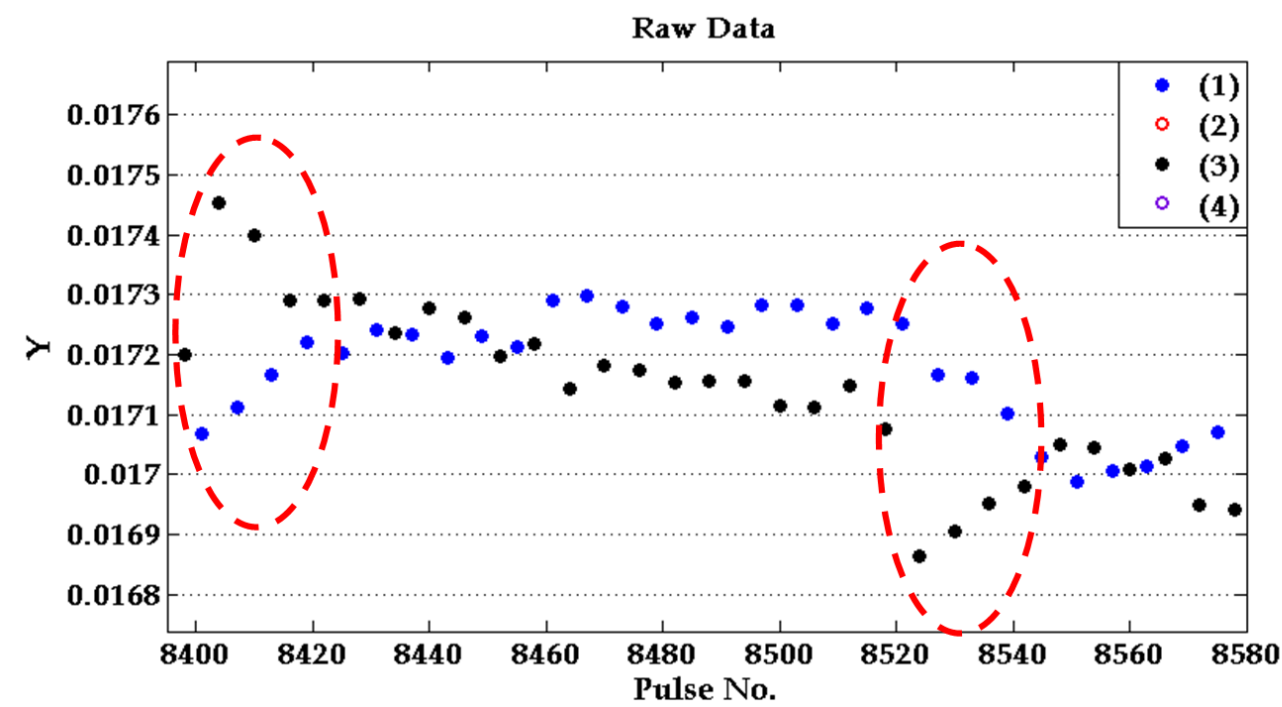

(b) Zoomed in from (a)

Figure 5.9: (a) Raw data from amplitude-ramp test in free-diffusion phantom with pure helium-3. Points (2) and (3) had a diffusion gradient before the DAQ in the TR period, and points (1) and (4) had a diffusion gradient after the DAQ in the TR period. (b) Zoomed in to show the transition between amplitude steps requires several pulses (indicated by red ovals) to reach the steady-state signal level. 


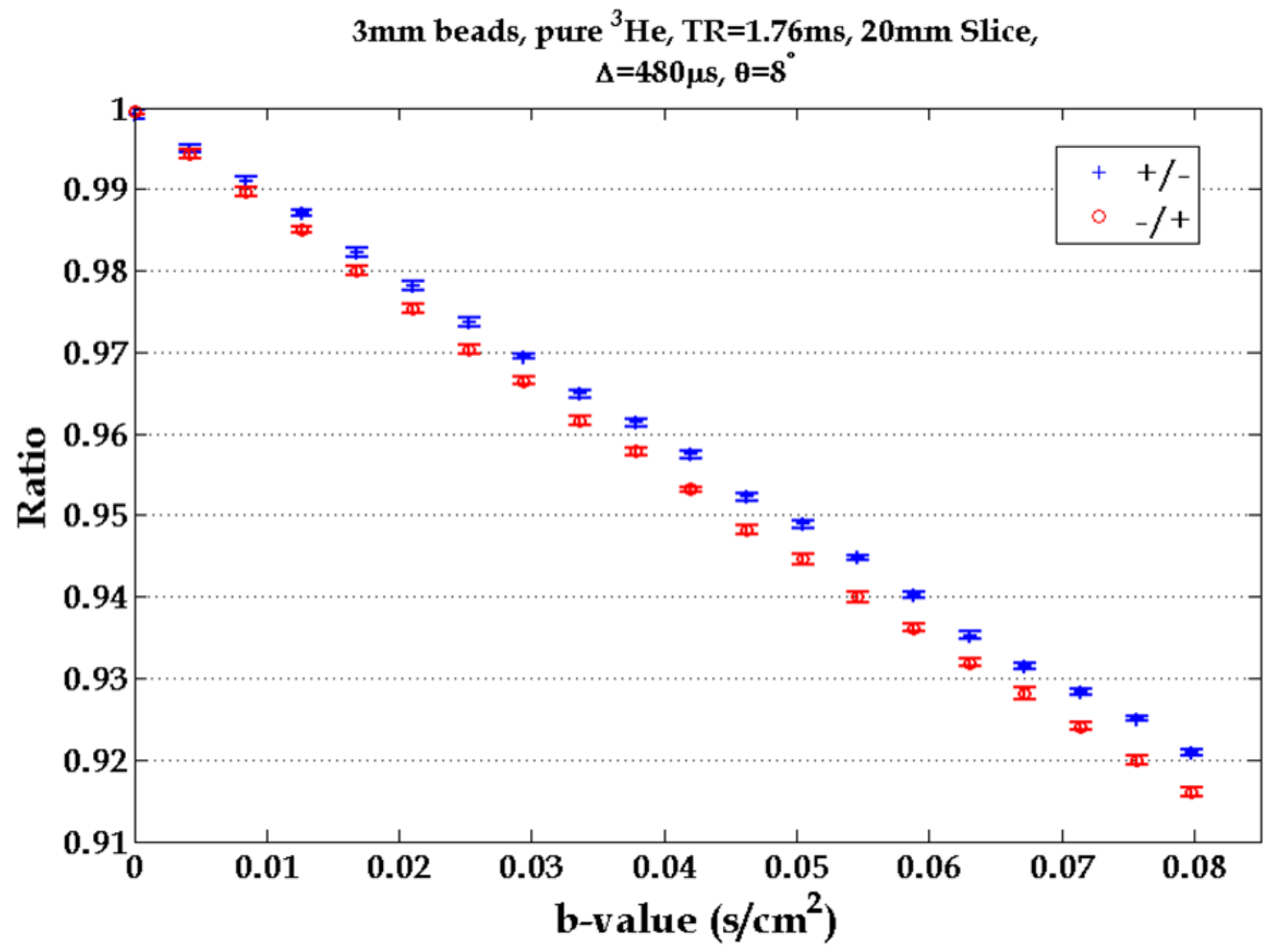

Figure 5.10: Ratio versus b-value for each gradient polarity from amplitude-ramp test in $3 \mathrm{~mm}$-bead phantom filled with pure helium-3. The positive-polarity diffusion gradients (blue crosses) produce a smaller steady-state signal ratio than the negative polarity diffusion gradients (red circles). 


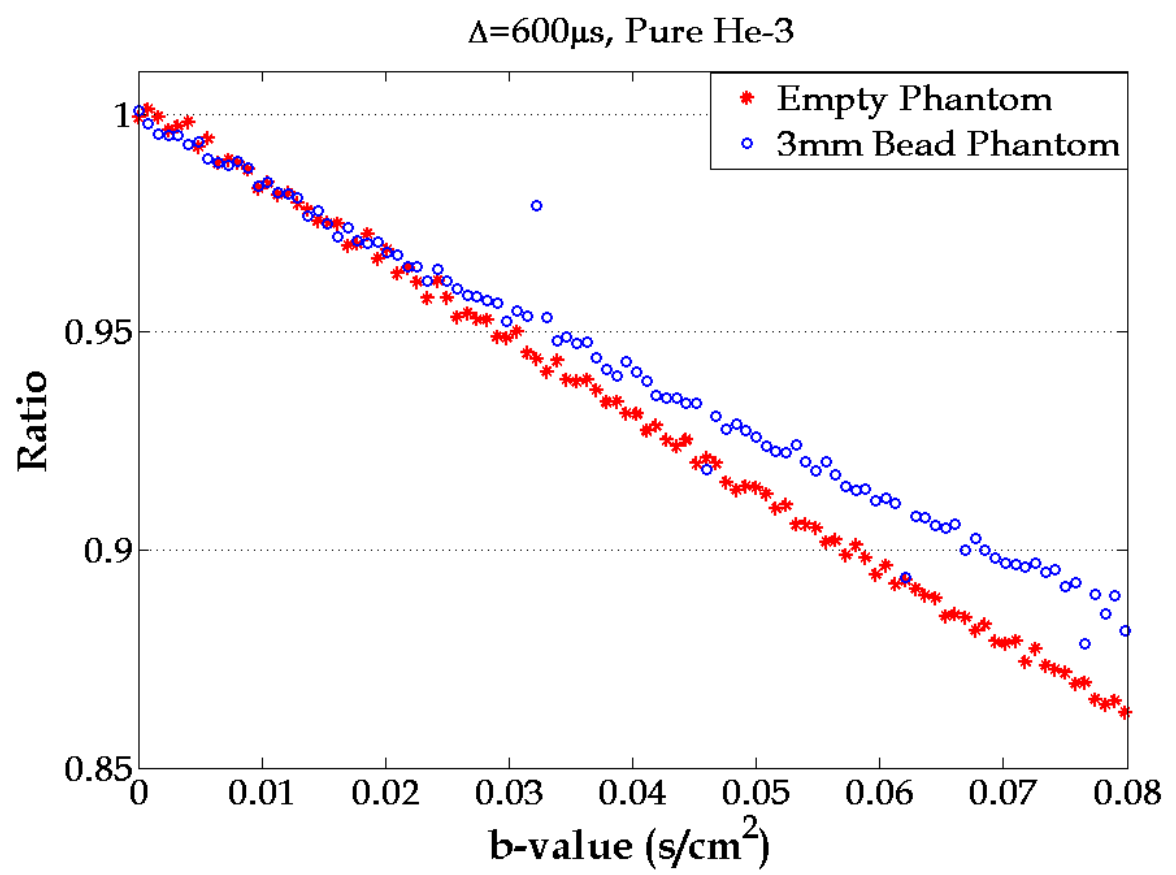

(a) Signal Ratios

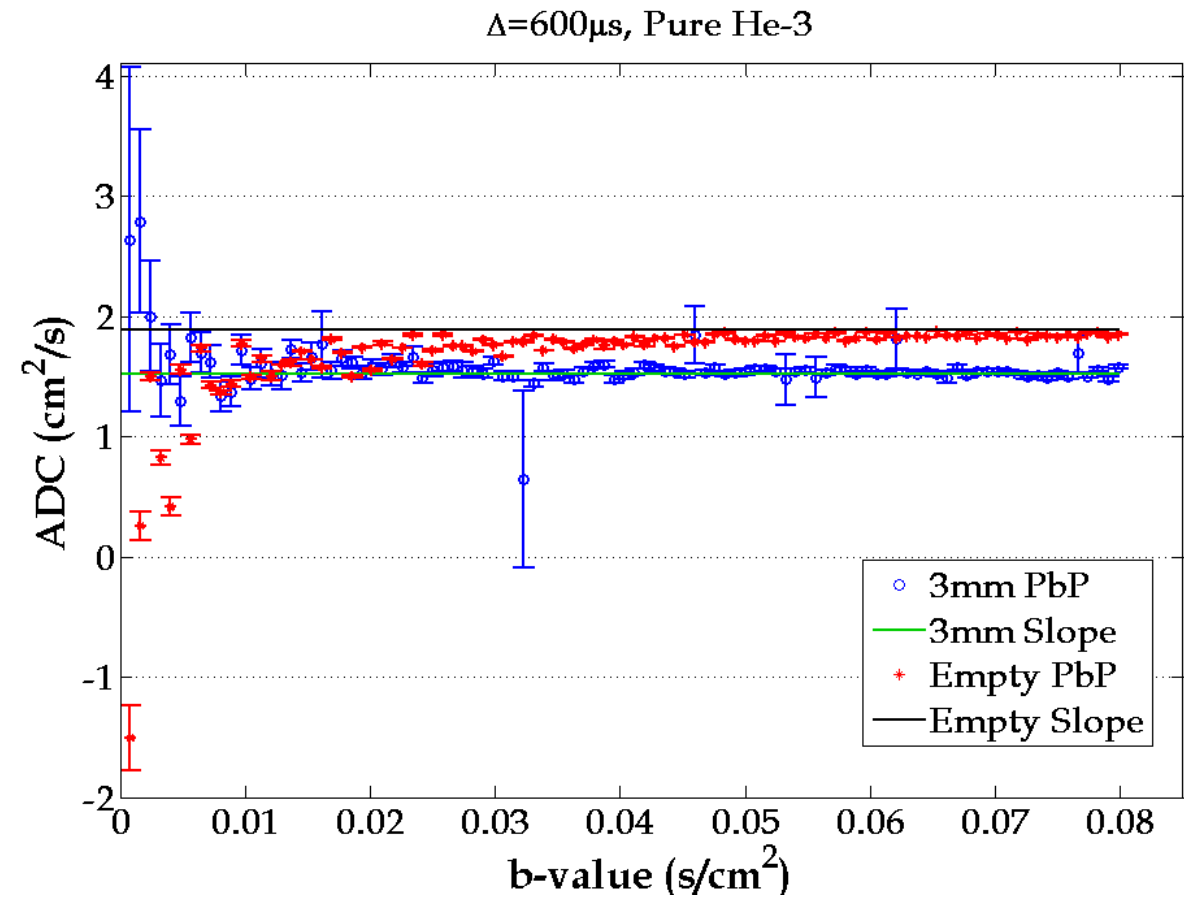

(b) ADCs

Figure 5.11: (a) Ratio versus b-value in $3 \mathrm{~mm}$-bead phantom and free diffusion phantom filled with pure helium-3 measured at $\Delta=600 \mu \mathrm{s}$. (b) ADC values calculated from natural log of ratios in (a) point-by-point ( $\mathrm{PbP}$, points) and from the slope of a linear fit to all of the data (lines) for each phantom. The values from both methods are similar in the $3 \mathrm{~mm}$-bead phantom, whereas in the free-diffusion phantom the point-by-point ADCs are systematically lower than the slope ADC value. 


\subsection{Time-dependent Diffusion Measurements in Bead Phantoms}

Using the results of the previous section, we designed a diffusion-weighted SSFP pulse sequence to test the accuracy of measured ADC values in all three of our glass bead phantoms. We measured helium-3 diffusion at increasing diffusion times with the Before/After diffusion-weighted SSFP pulse sequence to map out the time dependence of the diffusion coefficient. The diffusion time, diffusion gradient amplitude, and b-value evolution are shown in Figure 5.12. The longest diffusion times measured were capable of generating significant diffusion attenuation at the maximum gradient amplitude accessible by the MRI scanner. The high diffusion attenuation had the potential to diminish our signal before all of the diffusion times had been measured. To prevent this, we limited the gradient amplitude so that the largest $b$-value applied during the scan was $b=0.08 \mathrm{~s} / \mathrm{cm}^{2}$, resulting in smaller diffusion amplitudes for the longest $\Delta$ values, as shown in Figure 5.12b.

The pulse sequence parameters used were $T R=3.64 \mathrm{~ms}, \theta=6^{\circ}$, excitation $R F$ pulse duration $=100 \mu \mathrm{s}$, and we measured the signal in a transverse slice $20 \mathrm{~mm}$ thick. Measurements were made from $\Delta=300 \rightarrow 800 \mu$ s in $20 \mu$ s increments, and the diffusion gradient timing series of (1) after, (2) before, (3) before, (4) after was repeated twenty times using the positive diffusion-gradient polarity, then ten times with the negative polarity. The first ten repetitions at the positive diffusiongradient polarity were sufficient for the signal level to reach the steady state, and were therefore not included in the ADC analysis. Ratios were calculated identically to the amplitude-stepped acquisitions described in Section 5.5. The ADC was 


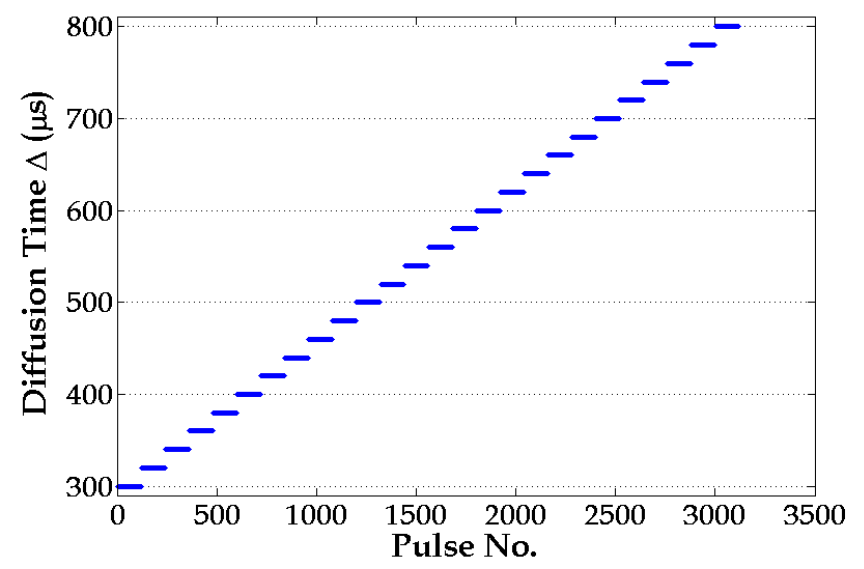

(a) Diffusion Time

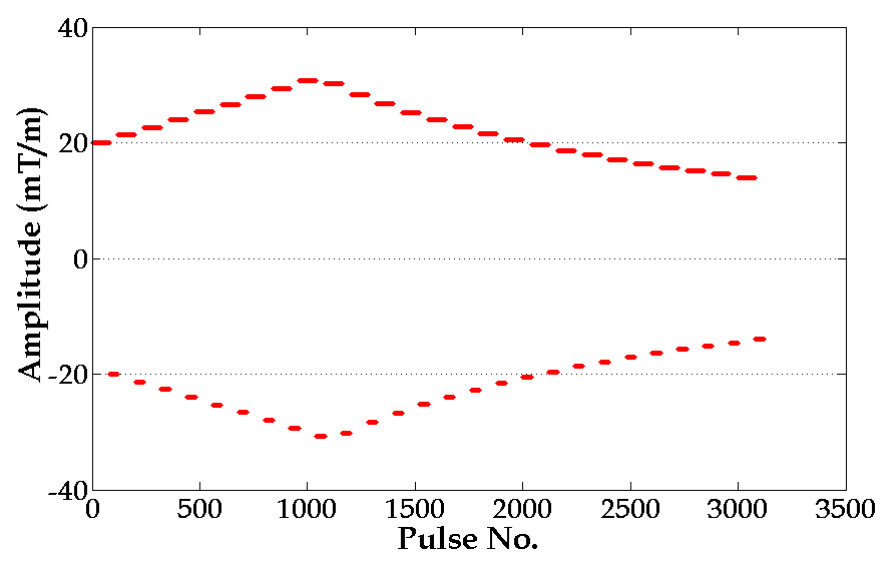

(b) Diffusion Amplitude

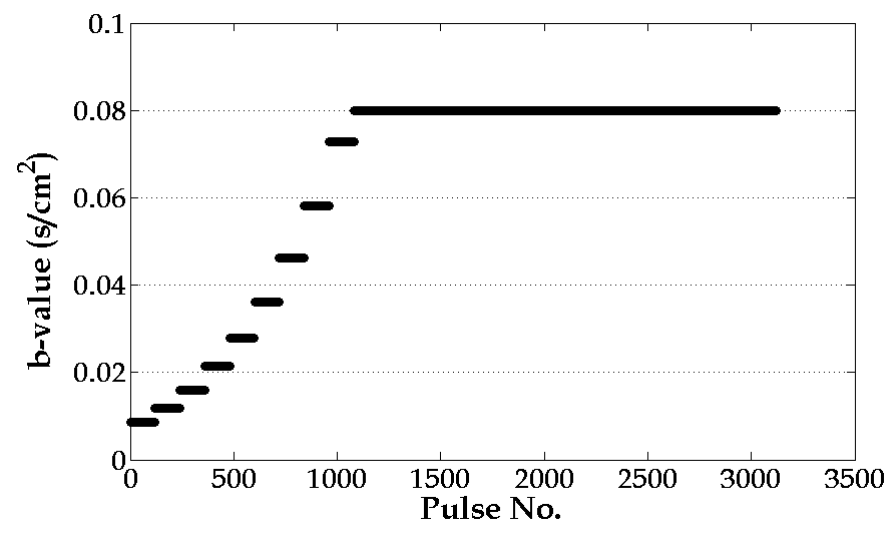

(c) b-value

Figure 5.12: Diffusion gradient progression for multiple diffusion time ADC measurements in the bead phantoms. The $\Delta$ values were increased throughout the scan, and the amplitude was calculated to be the maximum allowed by the scanner, unless the b-value was to be greater than $b=0.08 \mathrm{~s} / \mathrm{cm}^{2}$, then the amplitude was set to ensure $b=0.08 \mathrm{~s} / \mathrm{cm}^{2}$. 
calculated from each of the average ratios as follows:

$$
A D C(\Delta)=-\frac{\log R(\Delta)}{b(\Delta)}
$$

The ADC values are plotted vs $\sqrt{\Delta}$ in Figure 5.13, along with data acquired in the free-diffusion phantom $\left(D_{0}(\operatorname{avg})=1.91 \mathrm{~cm}^{2} / \mathrm{s}\right)$ and the theoretical tortuosity limit $\left(\sqrt{0.39} * D_{0}(\operatorname{avg})\right)$.

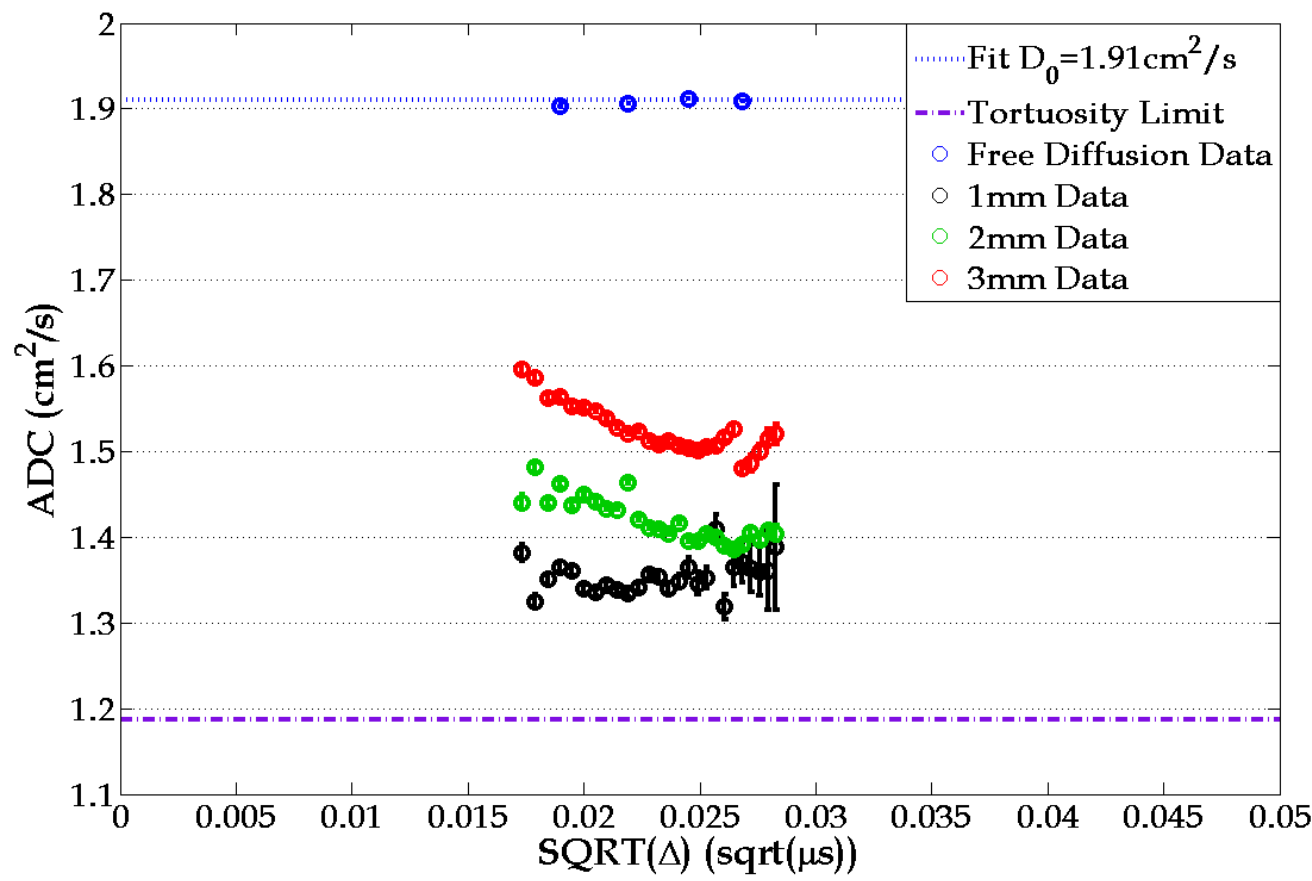

Figure 5.13: ADC measurements of pure helium-3 in glass-bead phantoms. The free-diffusion line is plotted at $D_{0}=1.91 \mathrm{~cm}^{2} / \mathrm{s}$, the average of the measurements made in the empty phantom. The tortuosity limit line was generated using the theoretical minimum porosity, $\phi=0.39$.

Each of the phantoms shows the expected time dependence in the diffusion coefficient, and the different structures are clearly separated in this measurement regime. There are systematic increases in the ADC for the largest values of $\Delta$ in each of the bead phantoms. We believe this increase is due to systematics caused by the low diffusion amplitudes applied to restrict the maximum b-value 
to $b=0.08 \mathrm{~s} / \mathrm{cm}^{2}$. In this region, it is possible that the background gradients due to susceptibility differences between the glass beads and the surrounding air are larger than the applied diffusion gradients. The background gradients would add to the net diffusion attenuation, artificially increasing the measured ADC as seen in our data. Lastly, a discontinuity can be observed in the data taken in the $3 \mathrm{~mm}$-bead phantom. Due to the steady-state nature of the data acquisition, this discontinuity is likely due to some anomaly that disturbed the steady state, but it isn't possible to say more without repeating the experiment.

As mentioned previously, the theoretical porosity of dense random-packed spheres is $\phi=0.39$. However, our beads were likely less densely packed than the theoretical ideal, as there appeared to be some movement in the pack when the phantoms were shaken. A non-ideal bead pack would increase $\phi$ from the theoretical value. We had the ability to measure the diameters of beads from the stock used to fill the phantoms, and the measured diameters were: $d(3)=3.08 \pm 0.02 \mathrm{~mm}$, $d(2)=1.98 \pm 0.14 \mathrm{~mm}$, and $d(1)=1.13 \pm 0.05 \mathrm{~mm}$. Using our measured values of $D_{0}$ and $d$, we fit our ADC values to the Padé equation to calculate a value of porosity for each phantom using:

$$
D(\Delta)=D_{0}\left(1-(1-\sqrt{\phi}) * \frac{\frac{4}{9 \sqrt{\pi}} \frac{6(1-\phi)}{d \phi} \sqrt{D_{0} \Delta}+(1-\sqrt{\phi}) \frac{D_{0} \Delta}{\left(L_{p} b\right)^{2}}}{(1-\sqrt{\phi})+\frac{4}{9 \sqrt{\pi}} \frac{6(1-\phi)}{d \phi} \sqrt{D_{0} \Delta}+(1-\sqrt{\phi}) \frac{D_{0} \Delta}{\left(L_{p} b\right)^{2}}}\right) .
$$

The measured ADC values and corresponding Padé fits are shown in Figure 5.14, and the input parameters and fit values are listed in Table 5.2.

The Padé curves match the ADC values for each bead phantom. The largest possible porosity for a close-pack of spheres occurs in a simple cubic lattice pack, with $\phi=0.52$ [80]. The $\phi$ values from the Pade fit to our data were well below the upper limit, and closer to the theoretical dense pack which was consistent with our 


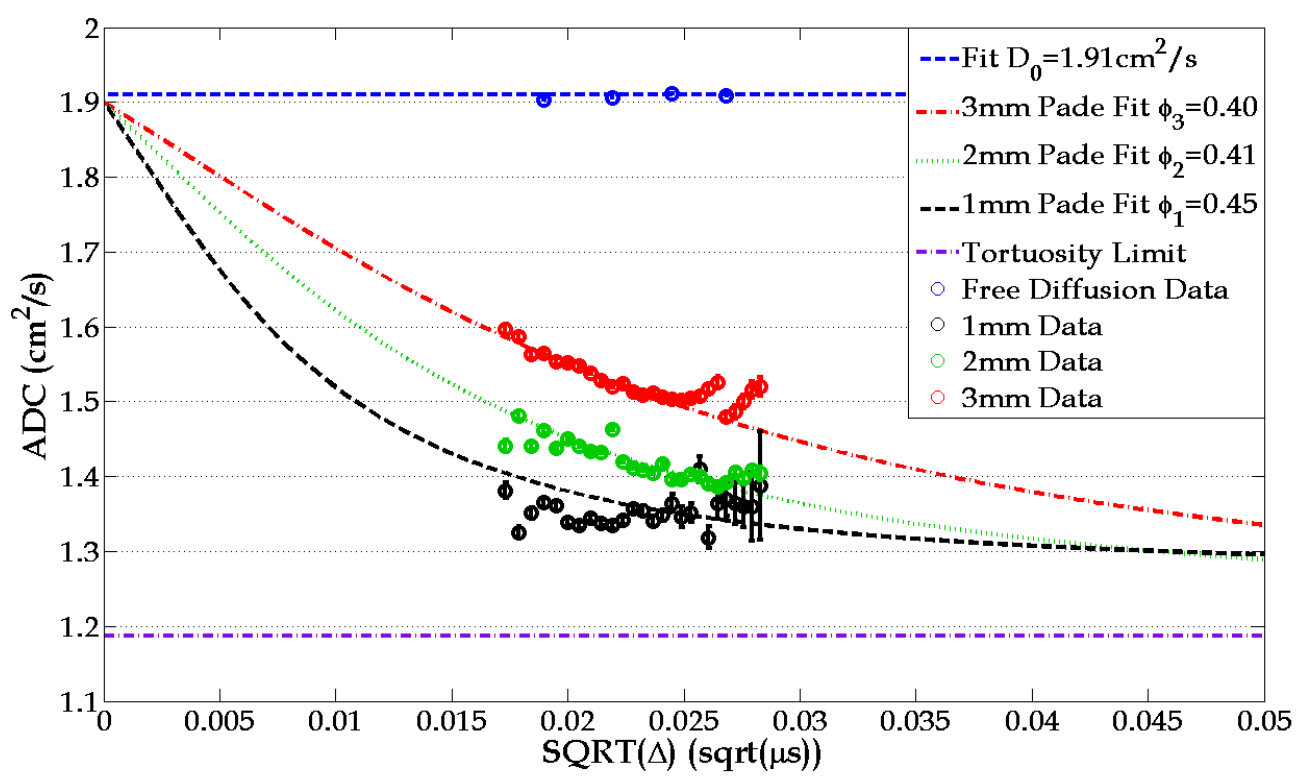

Figure 5.14: ADC data and Padé curves for pure helium-3 in glass bead phantoms. The free diffusion line is plotted at $D_{0}=1.91 \mathrm{~cm}^{2} / \mathrm{s}$, the value determined by the average of measurements made in the empty phantom. The tortuosity limit was generated using the theoretical minimum porosity, $\phi=0.39$. The Padé curves are fit to the porosity of each phantom.

observation of the phantoms. The $1 \mathrm{~mm}$-bead phantom had the largest calculated porosity $\phi_{1}=0.425$, and that phantom indeed had the most amount of mobility in the beads under agitation.

Our conclusion from the global test of the Before/After diffusion-weighted SSFP pulse sequence is that the ADC was being accurately measured with this technique. We observed systematic effects related to gradient polarity, as well as effects in the presence of low gradient amplitudes. The pulse sequence was designed to incorporate measurements of both diffusion gradient polarities and average the results, which appear to have successfully eliminated systematic errors due to polarity effects. The systematic effects in the instances of low gradient amplitudes are likely related to interference from susceptibility interfaces. Such effects should be much smaller in vivo, as the susceptibility difference is smaller between 


\begin{tabular}{|c|c|c|c|c|}
\hline & \multicolumn{3}{|c|}{ Input Parameters } & Output Parameter \\
\hline Bead Size & $L_{p}$ & $D_{0}$ & Diameter & $\phi$ \\
\hline $1 \mathrm{~mm}$ & 0.132 & $1.91 \mathrm{~cm}^{2} / \mathrm{s}$ & $1.13 \mathrm{~mm}$ & $0.425 \pm 0.003$ \\
\hline $2 \mathrm{~mm}$ & 0.132 & $1.91 \mathrm{~cm}^{2} / \mathrm{s}$ & $1.98 \mathrm{~mm}$ & $0.405 \pm 0.002$ \\
\hline $3 \mathrm{~mm}$ & 0.132 & $1.91 \mathrm{~cm}^{2} / \mathrm{s}$ & $3.08 \mathrm{~mm}$ & $0.398 \pm 0.002$ \\
\hline
\end{tabular}

Table 5.2: Padé equation parameter values for the bead phantoms. The value of the Padé length measured by Mair et al and $D_{0}=1.91 \mathrm{~cm}^{2} / \mathrm{s}$ measured in the freediffusion phantom were used in the fit. An independent measurement of bead diameters was used to determine $d$ for each phantom. The data was fit to the Padé equation (Equation 5.14), with the porosity $\phi$ as the free parameter for each bead phantom.

lung tissue $\left(\chi_{m}=-9 \mathrm{ppm}[65]\right)$ and air $\left(\chi_{m}=0.14 \mathrm{ppm}\right.$ [81]) than between glass $\left(\chi_{m}=-16 \mathrm{ppm}[82]\right)$ and air.

\subsection{Time-dependent Diffusion Measurements in $\mathbf{H u}-$ man Subjects}

We performed time-dependent ADC measurements in human subjects using the same Before/After diffusion-weighted SSFP pulse sequence used in the bead phantoms. We lowered the flip angle lowered to $\theta=2^{\circ}$ to reduce signal attenuation per pulse and ensure that the SNR did not decrease for the later values of diffusion time measured. The data were acquired in a sagittal slice centered in the right lung, selected to reduce the sensitivity to motion from the heart during the acquisition. The subjects were given a gas mixture consisting of $\sim 5-10 \%{ }^{3} \mathrm{He}$ and the balance medical-grade nitrogen. We measured the time-dependent ADC in two healthy subjects and one subject who had been diagnosed with Chronic Obstructive Pulmonary Disease (COPD). The ADC values measured are presented in Figure 5.15. 


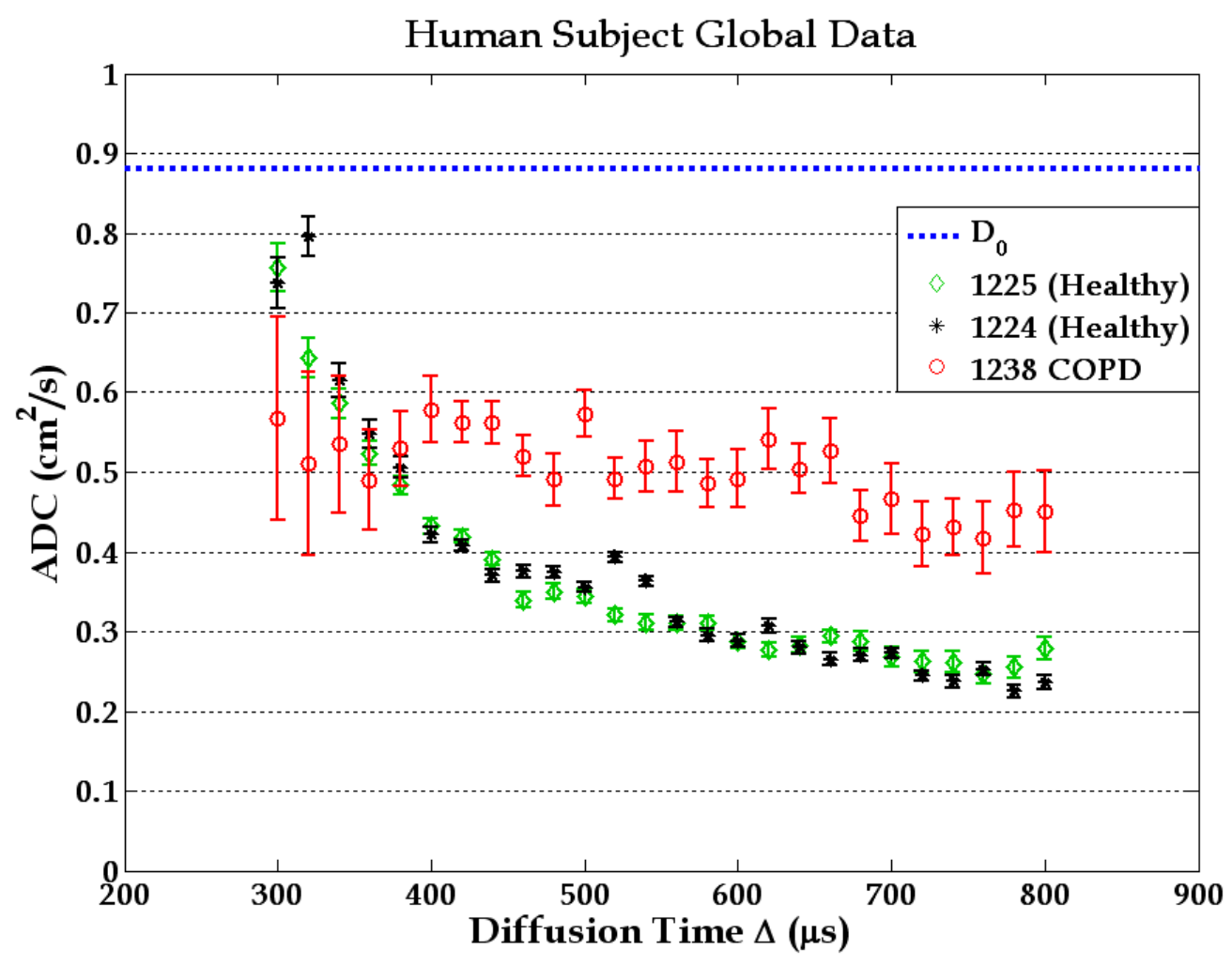

Figure 5.15: ADC v Diffusion Time in 3 subjects: two healthy volunteers, and one subject diagnosed with Chronic Obstructive Pulmonary Disease (COPD). The $A D C$ values are similar in the healthy subjects, and COPD subject shows a very different time-dependence of diffusivity. The results here suggest that the COPD subject has significantly different fine lung structure compared to the healthy subjects.

The healthy subjects (green diamonds and black stars) agree well with each other, each showing a decrease in ADC value as $\Delta$ increases. As we had expected, there was no systematic rise in the ADC values measured at long diffusion times in the lungs. The long $\Delta$ values had the same low diffusion gradient amplitudes that were applied in the bead phantoms. However the background gradients in the lung are smaller, so they contribute less additional diffusion attenutation.

The subject with COPD (red open circles) has a much flatter time-dependence of the diffusivity, suggesting that the fine structure of the lung tissue is significantly different from the healthy volunteers. The ADC values measured in the 
COPD subject with diffusion times above $\Delta=400 \mu$ s are larger than the healthy subject values, which is consistent with a less restrictive diffusion environment due to tissue destruction. The ADC values measured in the healthy subjects suggest that the diffusivity will reach the free diffusion coefficient $D_{0}=0.88 \mathrm{~cm}^{2} / \mathrm{s}$ at a diffusion time greater than $\Delta=0 \mathrm{~s}$. The ADC values measured in the COPD subject with diffusion times below $\Delta=400 \mu$ s are lower than with the healthy ADC values. Taking these two trends into consideration, it appears that the values of ADC measured at $\Delta<400 \mu$ s may be susceptible to systematic effects.

\subsection{Conclusions}

The results of our diffusion-weighted SSFP tests show that the Before/After diffusionweighted SSFP pulse sequence was able to accurately measure the small diffusionweighted attenuation produced at short diffusion times. In the glass bead phantoms, we were able to use the measured ADC values to calculate the porosity $\phi$ of the different phantoms.

In the test in human subjects, the time dependence of the diffusion coefficient was measured, but we do not have a theoretical description to compare the behavior to. The alveoli are not ideal spherical structures, and therefore the simplified geometric formulas which are applicable to bead phantoms, Equations 5.1 and 5.4, cannot be used to extract physiological parameters related to lung structure. However, the time-dependence of the diffusion coefficient was measured in healthy lungs and we determined that measurements of the diffusion coefficient at $\Delta>400 \mu$ s have a behavior similar to that seen in the bead phantoms. In the subject with COPD, the time-dependence of the diffusivity was markedly different, and the larger ADC values measured were consistent with a less restricted environment due to emphysema. 
The severity of tissue destruction associated with COPD varies regionally in the lung. Global ADC measurements, however, average over the regional variation and provide limited information about the disease. Diffusion-weighted images have the potential to contribute more to diagnosis and treatment development due to the regional information about lung structure. The global, time-dependent ADC measurements made here can be used as a guide to the values of ADC to expect when imaging at a specific value of the diffusion time $\Delta$, which is the subject of the next chapter. 


\section{Chapter 6}

\section{Hyperpolarized Gas MRI with Diffusion-weighted SSFP Pulse}

\section{Sequences}

Our global experiments in the previous chapter demonstrated that we are able to accurately measure free and restricted diffusivities using a diffusion-weighted SSFP pulse sequence. However for assessing lung structure changes due to emphysema, global measurements offer incomplete information by averaging over regional differences in air space geometry. By making an ADC map from diffusionweighted images, regional information about diffusivity can be obtained. In this chapter, we use diffusion-weighted SSFP pulse sequences to make ADC maps at short diffusion times, which have the potential to be more sensitive to small changes in lung structure.

We created imaging pulse sequences based on two of the diffusion-weighted SSFP pulse sequences developed in Chapter 4, and used them to acquire ADC maps in human volunteers and in our free-diffusion phantom. The Before/ After 
SSFP Diffusion pulse sequence provides ADC measurements that are robust against the effects of off-resonant spins, but the minimum timing requirements result in a relatively long repetition time (TR) for an SSFP pulse sequence. The long repetition time could potentially lead to off-resonance precession angles of $\beta=180^{\circ}$ per TR in some regions of the image, which would result in a loss of signal in those regions. The Group of Three diffusion-weighted SSFP pulse sequence can have a much shorter TR since the diffusion gradients and the DAQ window are in separate TR periods. However, the measured diffusion-weighted signal ratio can be distorted by the effects of off-resonant spins when using this sequence. We evaluated the images and ADC maps produced using both the Group of Three and the Before/After SSFP imaging pulse sequence to assess the degree to which offresonant magnetization affects the results in each case.

\subsection{SSFP Imaging Considerations}

Our global SSFP diffusion measurements in Chapter 5 were repeated many times throughout the acquisition to ensure that the magnetization was in steady state and to provide a large number of measurements that could be averaged to reduce statistical errors. To acquire an image, the repetition of RF excitations is used to sample k-space with imaging gradients. As discussed in Chapter 2, typical MR imaging is performed by acquiring one line of k-space per TR period, incrementing the phase-encode gradient size after each RF pulse, until the range of values necessary to make the image has been covered. In order to make diffusion measurements with our SSFP pulse sequences, we needed to acquire multiple images and combine them to form steady state ratios that gave diffusion measurements and canceled out baseline RF differences, similar to how data was combined for the non-imaging experiments in Chapter 5. 
If one were to acquire all of the data for each image serially, there would be a systematic signal difference between images due to the hyperpolarized magnetization decaying towards equilibrium. There could also be other differences between serially-acquired images, for example due to motion of the subject. Therefore, the multiple image acquisitions were interleaved line-by-line to minimize the time between the images. For each line of k-space, identical imaging gradients were repeated the necessary number of times (six for Group of Three, four for Before/After) before incrementing to the next line. Because we needed the magnetization to be in steady-state to achieve the desired diffusivity contrast, we also added an extra 10-20 "warm-up" RF excitations to the beginning of each scan before we began acquiring data, to give the magnetization time to evolve into the steady state.

\subsection{Experimental Methods}

To start each measurement session, helium-3 was polarized using our Hybrid Helium Polarizer (see Chapter 3) to $\sim 60 \%$. In phantom imaging sessions, the helium3 dosing procedure was performed in the same manner as described in Chapter 5. For imaging sessions with human subjects, a dosing bag was attached to the polarizer outlet via a short length of $\frac{1}{4}$-inch Tygon tubing, evacuated, and pre-filled with a measured amount of medical-grade nitrogen gas. The subject was positioned supine in the 1.5 T MR scanner (Avanto, Siemens Medical Solutions, Malvern PA) in an RF coil tuned to the helium-3 resonance (Rapid Biomedical, Rimpar Germany), and conventional ${ }^{1} \mathrm{H}$ images were acquired to determine the lung position. A measured dose of ${ }^{3} \mathrm{He}$ was then dispensed into the dosing bag, the tube was disconnected from the polarizer outlet, and the bag was brought into the scanner room. The subject inhaled the mixture of nitrogen and polarized helium-3 from 
the dosing bag through the $\frac{1}{4}$-inch tube. The imaging scan was then performed at breath-hold.

The data were processed using Matlab software. To create an ADC map from the raw data, first a multidimensional Fourier transform was performed to convert the k-space data into four separate helium-3 images. The appropriate diffusionweighted image ratio was then calculated pixel-by-pixel from the helium-3 images. In order to mask out background pixels, the image ratio was only calculated at pixel locations for which the signal magnitude exceeded a calculated threshold, chosen here to be $a=0.1 *$ (Max Signal in Image). From the ratio map $R$, an ADC map was calculated as follows:

$$
A D C=-\frac{\log R}{b}
$$

where $b$ was the $b$-value of the diffusion-sensitizing gradient.

\subsection{Group of Three Imaging Test}

Our previous global tests of the Group of Three diffusion-weighted SSFP pulse sequence produced accurate ADC measurements in the free-diffusion phantom, but implausible ADC values in the $3 \mathrm{~mm}$-bead phantom. We believe the presence of susceptibility-induced background gradients caused the inaccurate measurements. Due to the reduced susceptibility mismatch between lung tissue and air compared to glass and air, we expected the size of the susceptibility-induced background gradients in the lung airspaces to be smaller than those in our bead phantoms. Therefore the range of off-resonance frequencies and thus the resulting effects on the steady-state signal ratio were also expected to be smaller in vivo.

Imaging gradients were incorporated into the Group of Three diffusion-weighted 

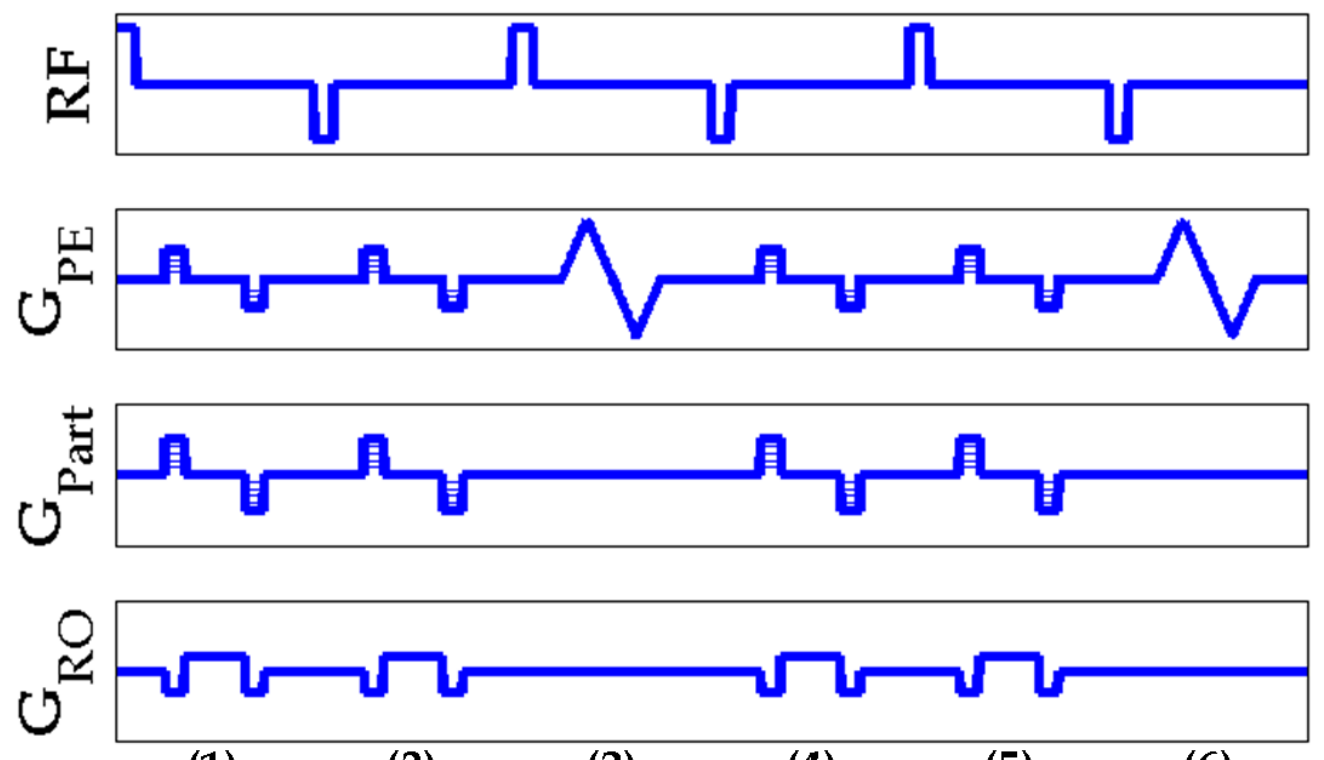

(1)

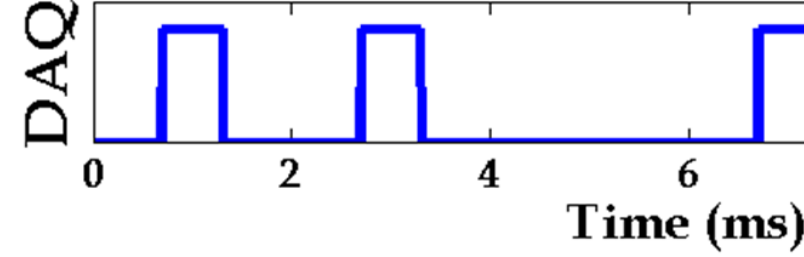

Figure 6.1: Imaging implementation of the Group of Three SSFP diffusionweighted pulse sequence. Four images are interleaved, with diffusion-sensitizing gradients in TR periods with no data acquisition.

SSFP pulse sequence during TR periods containing the data acquisition windows as shown in Figure 6.1. The pulse sequence imaging parameters were chosen to yield three-dimensional images with isotropic resolution. As described in Chapter 2 , there are two ways obtain three dimensional k-space information: sliceselective imaging and partition-encoded imaging. In slice selective imaging, a gradient is applied during the excitation RF pulse which results in only a portion of the spins being tipped into the transverse plane. In partition-encoded imaging shown in Figure 6.1, the third gradient axis (partition) is stepped through similarly to the phase-encode gradient. Images acquired in this matter can be viewed as slices from any of the three principal axes: phase-encode, read-out, or partition. 
The Group of Three SSFP diffusion-weighted imaging (DWI) pulse sequence interleaved four image acquisitions and two diffusion-sensitizing gradient TR periods at each phase-encode and partition step. By averaging images with opposite RF phase angles and similar diffusion-weighting, the baseline signal asymmetry due to RF phase was cancelled out:

$$
R=\frac{I(2)+I(5)}{I(1)+I(4)}
$$

where $I(1)$ is the image formed in the first TR period per line, etc. and $R$ is the diffusion-weighted signal ratio.

We used the Group of Three diffusion-weighted SSFP imaging pulse sequence described above to make diffusion measurements in a healthy human subject. For this test, the inhaled dose consisted of $300 \mathrm{~mL}$ of helium-3 was diluted with $1.6 \mathrm{~L}$ of medical grade nitrogen, which lowers the free diffusion coefficient of the helium3 gas to $D_{0}=0.88 \mathrm{~cm}^{2} / \mathrm{s}$. The pulse sequence parameters were: $T R=1.22 \mathrm{~ms}$, $\theta=6^{\circ}, 3 \mathrm{D}$ isotropic resolution $7.5 \times 7.5 \times 7.5 \mathrm{~mm}$, RF pulse width $=240 \mu \mathrm{s}$, a readout bandwidth of $B W=2440 \mathrm{~Hz} / \mathrm{px}, \Delta=560 \mu \mathrm{s}, G_{\text {Diff }}=32 \mathrm{mT} / \mathrm{m}$, and a b-value of $b=0.23 \mathrm{~s} / \mathrm{cm}^{2}$. The raw images could be reconstructed in any of the three imaging planes: coronal (viewed from the front of the subject), sagittal (viewed from the side of the subject), and transverse (viewed from the top of the subject). Central segments in each of these orientations from the first of the four interleaved images are shown in Figure 6.2a.

The raw lung ventilation images from the test of the Group of Three SSFP DWI do not have any obvious stripes or bands of reduced signal, suggesting that the repetition time $T R=1.22 \mathrm{~ms}$ was short enough to prevent off-resonant phase accumulation from reaching $\beta=180^{\circ}$ per TR at any point. The different levels of signal intensity (particularly evident top-to-bottom in the sagittal slice) over the 
lung are consistent with expected ventilation inhalation, and do not appear to be pathological. The four interleaved images were combined pixel-by-pixel according to Equation 6.2 to produce a ratio map, shown in Figure 6.2b.

In some regions the signal ratio is greater than one- a reversal of the expected contrast due to diffusion. The regions with a ratio above one are at the top and bottom of the lungs, which are farthest from the center of the scanner bore where the magnetic field is most uniform. Based on our simulations of the Group of Three SSFP pulse sequence, a signal ratio greater than one can be caused by the presence of off-resonant spins with a precession angle of $\beta>60^{\circ}$ per TR, as discussed in Section 4.6.3. To test this prediction, we conducted an experiment in the free-diffusion phantom in which we intentionally induced off-resonant precession by increasing the system frequency on the scanner from the measured value $f_{0}$. For instance, a precession angle of $\beta \approx 60^{\circ}$ per TR can be achieved using a resonant frequency offset $\Delta f_{0}$,

$$
\Delta f_{0}=\frac{1}{T R} * \frac{60}{360}=\frac{1}{1.46 \mathrm{~ms}} * 0.167 \approx 110 \mathrm{~Hz}
$$

That is, if $\Delta f_{0}=110 \mathrm{~Hz}$ the spins at the center of the scanner would be expected to precess $\beta \approx 60^{\circ}$ per TR. According to our simulations, any spins with larger precession angles should generate signal ratios greater than one, and spins with smaller precession angles should generate signal ratios less than one. The pulse sequence parameters used for this imaging test were: $T R=1.46 \mathrm{~ms}, \theta=4^{\circ}$, 3D isotropic resolution $4 \times 4 \times 4 \mathrm{~mm}, \mathrm{RF}$ pulse width $=240 \mu \mathrm{s}$, a readout bandwidth of $B W=2440 \mathrm{~Hz} / \mathrm{px}, \Delta=480 \mu \mathrm{s}, G_{\text {Diff }}=32 \mathrm{mT} / \mathrm{m}$, and a b-value of $b=$ $0.09 \mathrm{~s} / \mathrm{cm}^{2}$. We acquired diffusion-weighted images at three frequency settings: $\Delta f_{0}=0 \mathrm{~Hz}, \Delta f_{0}=110 \mathrm{~Hz}$, and $\Delta f_{0}=130 \mathrm{~Hz}$. Signal ratio maps from the four images were generated as previously described and are shown in Figure 6.3.

The ratio maps from the frequency-centered acquisition $\left(\Delta f_{0}=0 \mathrm{~Hz}\right)$ have a 
uniform mean ratio that is less than one (Figure 6.3a), as expected. In the acquisition centered at $\Delta f_{0}=110 \mathrm{~Hz}$ (b), the ratio map shows values both greater than and less than one. The ratio maps from the $\Delta f_{0}=130 \mathrm{~Hz}$ acquisition have values that are all greater than one. The results of this test support our simulation results that the Group of Three diffusion-weighted SSFP pulse sequence produces signal ratios greater than one in the presence of off-resonant precession angles $\beta \geq 60^{\circ}$ per TR.

Looking again at our signal ratio maps from the human subject test (Figure 6.2b), the majority of the lung area has a ratio close to or less than one. It is only the top and bottom of the lung which seem to experience the large off-resonant precession angles. This feature suggests that the magnetic field variations which drive the precession are macroscopic variations, not microscopic gradients caused by susceptibility interfaces at air-tissue boundaries throughout the lung. Using the assumption that the off-resonance magnetization precession is at the transition point of $\beta=60^{\circ}$ per TR near the top of the lung where we see values cross over from less than one to greater than one, we can calculate the size of the scanner holding field variation that would lead to that amount of precession:

$$
\begin{gathered}
\Delta f_{0}\left(\beta=60^{\circ} / \mathrm{TR}\right)=110 \mathrm{~Hz}, \quad \frac{\gamma_{\mathrm{He} 3}}{2 \pi}=-32.43 \mathrm{MHz} / \mathrm{T}, \\
\Delta B_{0}=\frac{\Delta f_{0}}{\gamma / 2 \pi}=3.4 \mu \mathrm{T}, \\
\frac{\Delta B_{0}}{B_{0}}=\frac{3.4 \mu \mathrm{T}}{1.5 \mathrm{~T}}=2.3 \mathrm{ppm} .
\end{gathered}
$$

Our calculated field variation of $2.3 \mathrm{ppm}$ is close to the specified tolerance of the scanner of $\Delta B_{0} / B_{0}<1.5 \mathrm{ppm}$.

With a more uniform magnetic holding field, it is possible that the off-resonant precession could be reduced such that the Group of Three SSFP DWI pulse se- 
quence would produce purely diffusion-weighted maps, rather than the combination of diffusion-weighting and off-resonant precession-weighting we see with the current system. 

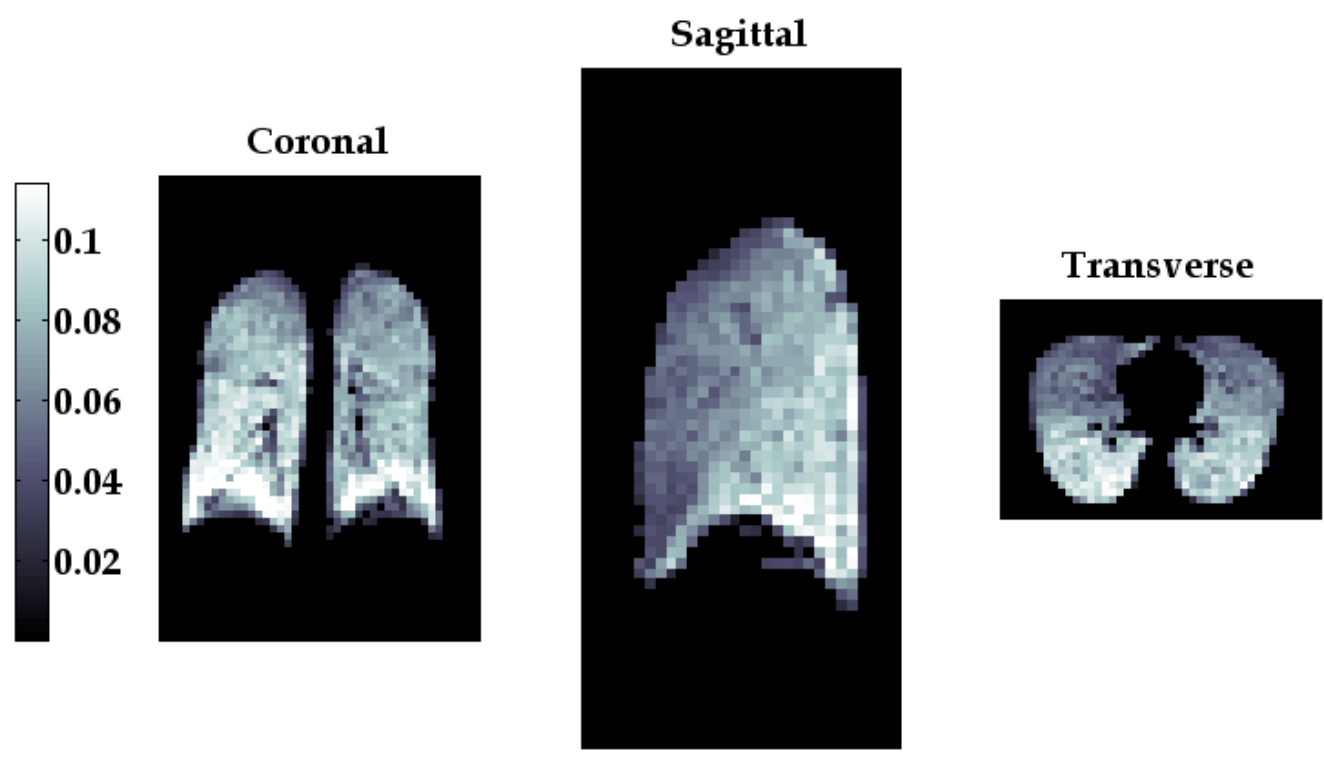

(a) Images

Sagittal:

Coronal:

$\mathrm{R}=1.051+/-0.191$
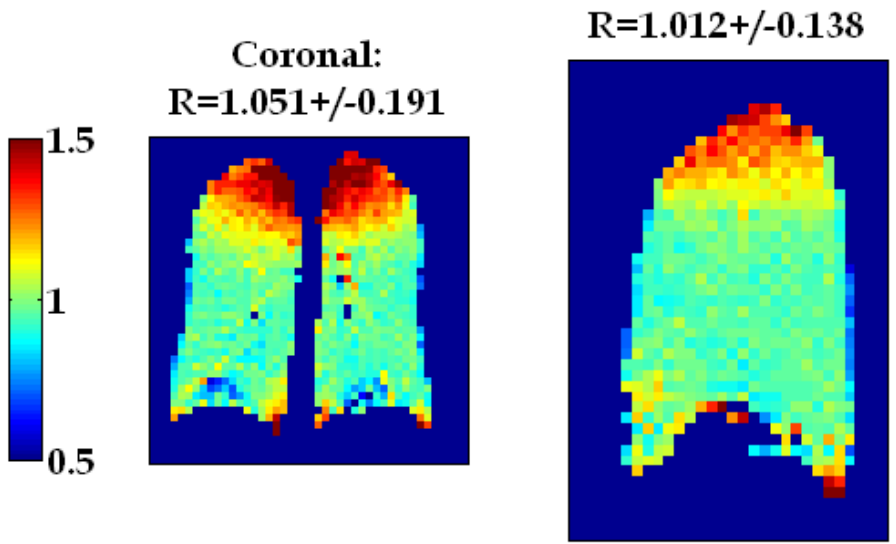

Transverse:

$\mathrm{R}=0.934+/-0.069$

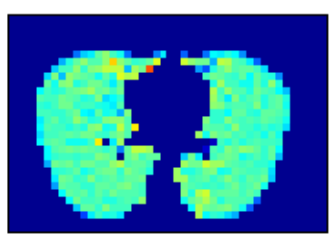

(b) Ratio Maps

Figure 6.2: Lung ventilation images (a) and signal ratio maps (b) made with the Group of Three SSFP DWI in healthy subject 1219. The raw images are from the first of the four interleaved images, and show a variation in signal intensity consistent with normal ventilation non-uniformity (particularly evident top-to-bottom in the sagittal and axial slices). The signal ratios are greater than one at the top and bottom of the lungs, which is the opposite of the expected diffusion-weighted contrast. 


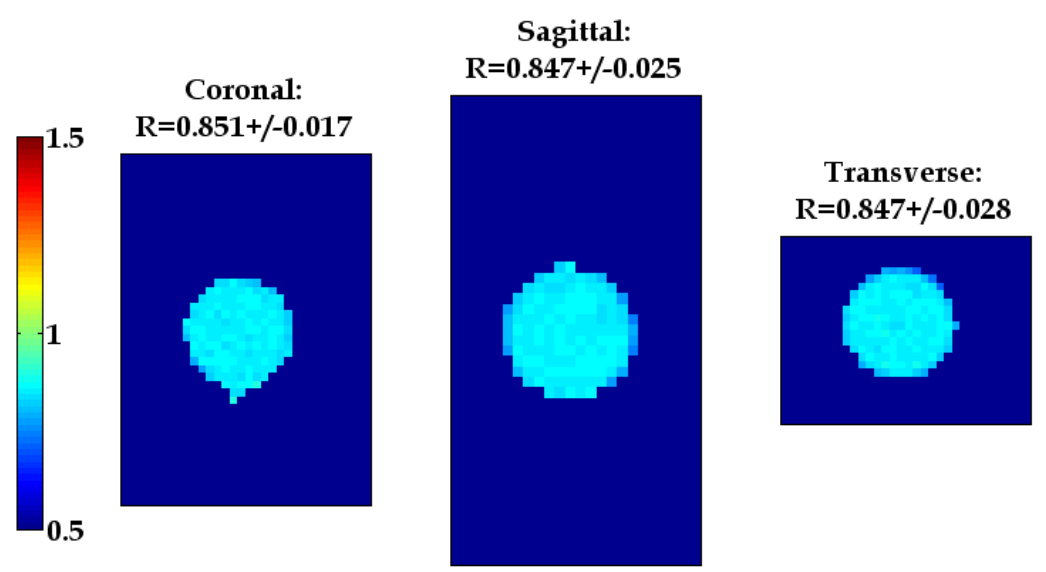

(a) Frequency-Centered Ratios

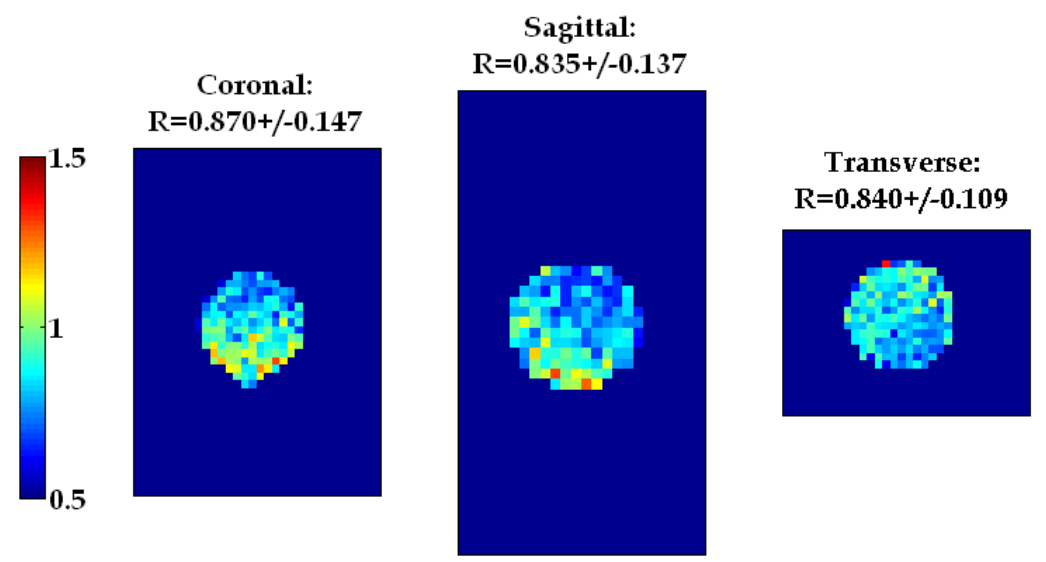

(b) $\Delta f_{0}=110 \mathrm{~Hz}$
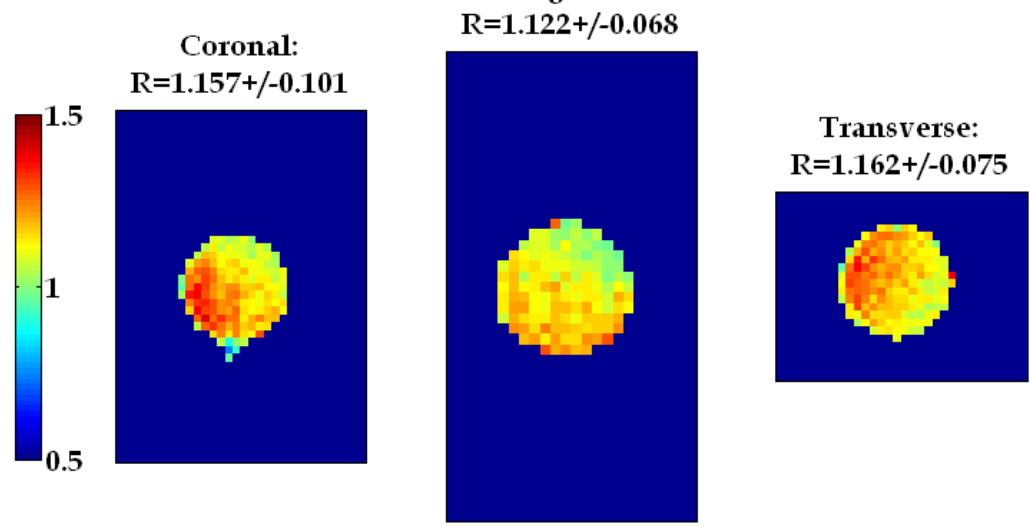

(c) $\Delta f_{0}=130 \mathrm{~Hz}$

Figure 6.3: Signal ratio maps and mean values from the (a) frequency-centered and (b)-(c) off-centered acquisitions using the Group of Three SSFP DWI pulse sequence, reconstructed in each imaging plane. In the frequency-centered acquisition (a), the signal ratios are less than one, which is the expected direction of contrast. In (b) some signal ratios are greater than 1 and others less than 1 , consistent with a range of precession angles that straddles the transition point of $\beta=60^{\circ}$ per TR. In (c), the signal ratios are all greater than 1 , which is the opposite of the expected contrast and indicative of precession angles of $\beta>60^{\circ}$ per TR. 


\subsection{Before/After SSFP DWI}
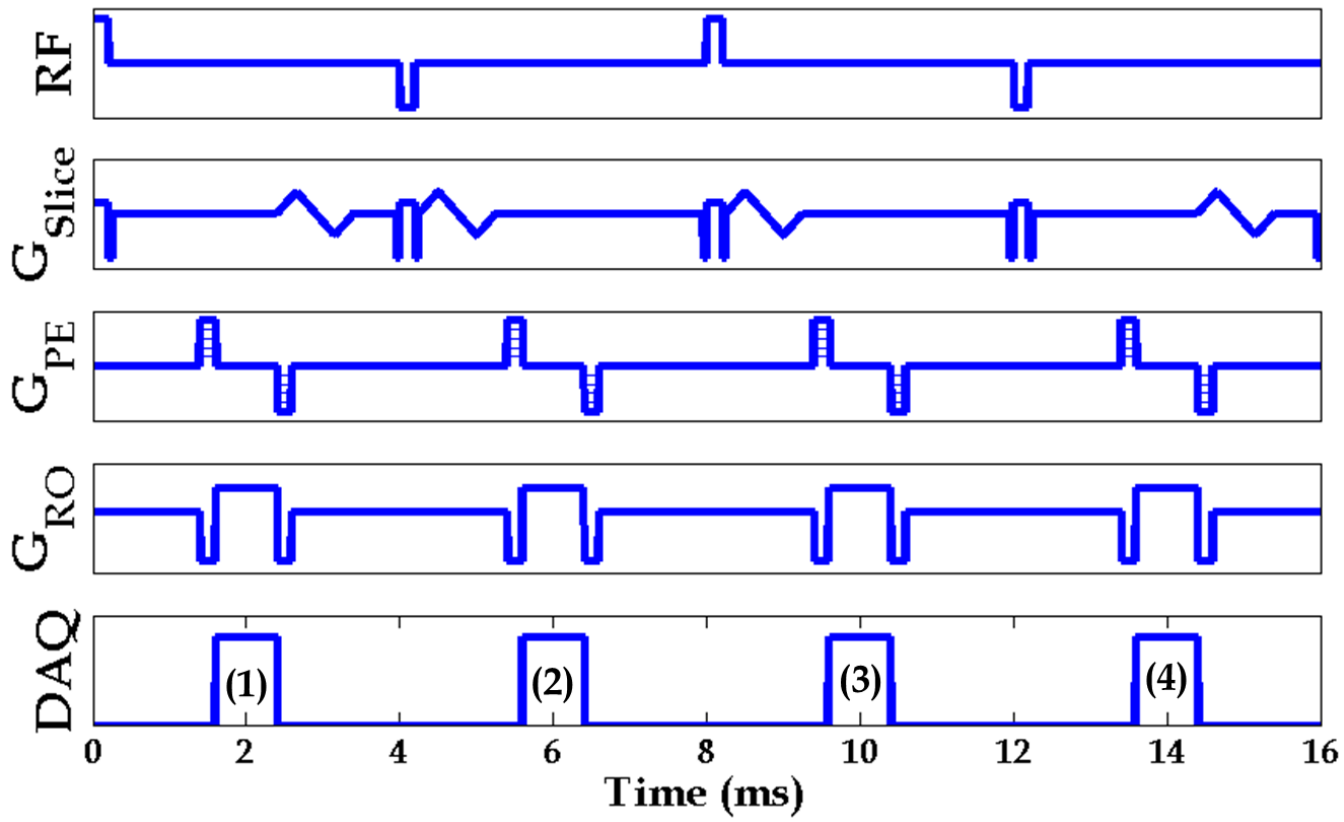

Figure 6.4: Imaging implementation of the Before/After SSFP diffusion-weighted pulse sequence. The sequence produces four interleaved images, with diffusionsensitizing gradients applied in the slice direction (1) after, (2) before, (3) before, and (4) after the data acquisition.

The Before/After diffusion-weighted SSFP pulse sequence was designed to accurately measure the diffusivity in the presence of off-resonant magnetization. We added slice-selective imaging gradients to the Before/After diffusion-weighted SSFP pulse sequence as shown in Figure 6.4. The diffusion-sensitizing gradients are in the same direction as the slice selective gradients. Like the Group of Three method, this sequence also produces four images but there are diffusion-sensitizing gradients, imaging gradients, and data acquisition windows in every TR period. Images 2 and 3 have the same diffusion-weighting but opposite RF phase angles, as do images 1 and 4 . The images are combined to produce a ratio map as follows:

$$
R=\frac{I(2)+I(3)}{I(1)+I(4)}
$$


We used our SSFP magnetization simulation from Chapter 4 to determine the optimal flip angle for the Before/After SSFP DWI pulse sequence for various degrees of diffusion attenuation. For a constant flip angle, the transverse magnetization generated by each RF excitation pulse declines throughout the acquisition. Because the magnetization evolution is coherent, the rate of decline depends on both the flip angle used and the rate of transverse relaxation. For a given rate of transverse relaxation, which is dominated by the diffusion-induced signal attenuation in our case, the optimal flip angle is the value that yields the highest average signal over the course of the acquisition. To determine this value, we calculated the size of transverse magnetization after 114 RF excitations, which corresponds to the central line of k-space data for each slice which consists of $228 \mathrm{RF}$ excitations (20 RF excitations for the magnetization to reach steady state and 52 phase-encode lines interleaved for each of the 4 images). The results of our simulation are plotted versus applied flip angle for varying values of diffusivity in Figure 6.5. Our simulation indicated that we should expect an optimal flip angle of $\theta \approx 27^{\circ}$ in the small lung airspaces $\left(D \sim 0.3 \mathrm{~cm}^{2} / \mathrm{s}\right)$, and $\theta \approx 20^{\circ}$ in the major airways which have essentially free diffusion ( $D \sim 0.9 \mathrm{~cm}^{2} / \mathrm{s}$ ). We used $\theta=20^{\circ}$ for our Before/After SSFP DWI pulse sequence.

The first Before/After SSFP DW images were taken in a healthy volunteer using the following parameters: RF pulse duration $=300 \mu \mathrm{s}, 2 \mathrm{D}$ imaging in $20 \mathrm{~mm}$ slices with in-plane resolution of $6.6 \times 6.6 \mathrm{~mm}$, and a readout bandwidth of $B W=$ $780 \mathrm{~Hz} / \mathrm{px}, \Delta=500 \mu \mathrm{s}, G_{\text {Diff }}=27 \mathrm{mT} / \mathrm{m}$, and $b=0.1 \mathrm{~s} / \mathrm{cm}^{2}$. The minimum repetition time achievable for the Before/After SSFP DWI pulse sequence using these parameters was $T R=4.2 \mathrm{~ms}$. The imaging procedure was the same as in the Group of Three imaging test, and the subject inhaled a mixture of $350 \mathrm{~mL}$ hyperpolarized helium-3 and $1.15 \mathrm{~L}$ medical grade nitrogen. Ventilation images 


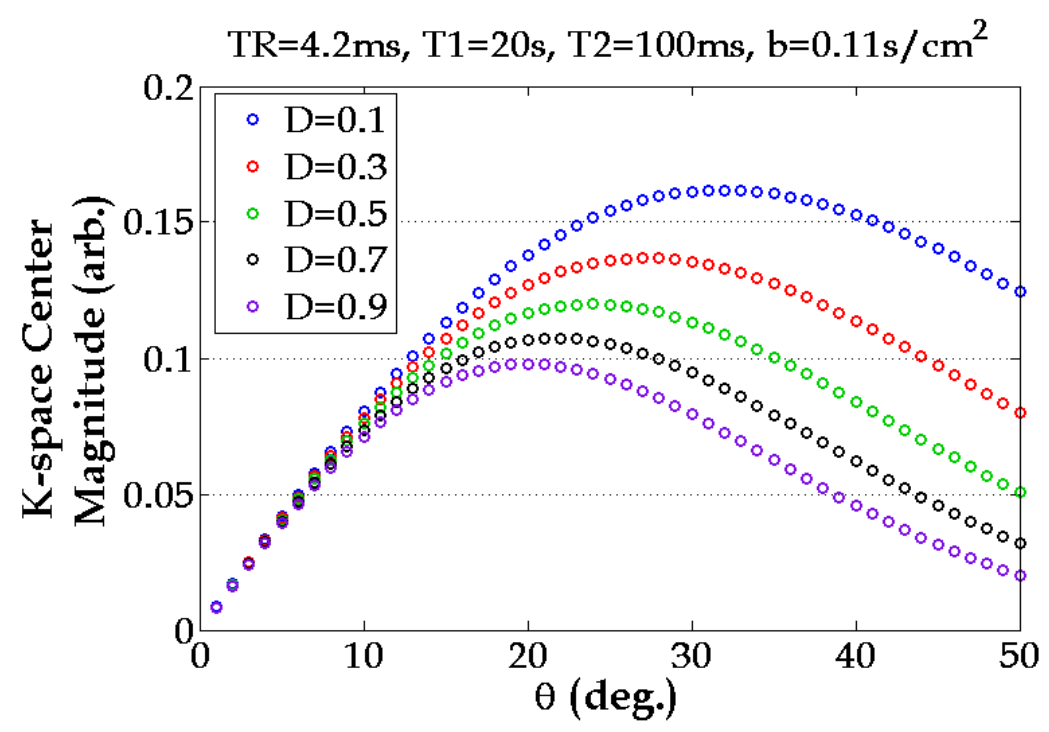

Figure 6.5: Simulation of the magnetization at the central line acquisition of the Before/After diffusion-weighted SSFP pulse sequence versus flip angle for different values of diffusivity $D$. Using dilute helium-3, the expected values of diffusivity at $\Delta=500 \mu$ s are $D \approx 0.3 \mathrm{~cm}^{2} / \mathrm{s}$ in the alveoli and $D \approx 0.8 \mathrm{~cm}^{2} / \mathrm{s}$ in the large airways.

and ADC maps from a central lung slice and a posterior lung slice are shown in Figure 6.6.

The ventilation images in Figure 6.6(a-b) produced by the Before/After SSFP DWI pulse sequence are uniform overall, however the central-slice ventilation image (a) has a dark stripe in the lower left lung near the diaphragm. This feature is consistent with signal drop-out caused by off-resonance precession due to susceptibility-induced gradients at the base of the lung. The ADC maps in Figure 6.6(c-d) show a uniformly low diffusivity in the lung parenchyma, $A D C \approx$ $0.26 \mathrm{~cm}^{2} / \mathrm{s}$. The combination of diffusivity and b-value applied here generated a steady state ratio:

$$
R=e^{-b * D}=e^{-0.1 * 0.26}=0.974
$$

In the trachea (see Fig. $6.6 \mathrm{c}$ ), the measured diffusivity $A D C \approx 0.8 \mathrm{~cm}^{2} / \mathrm{s}$ is close to the free diffusion coefficient of helium-3 dilute in air. 


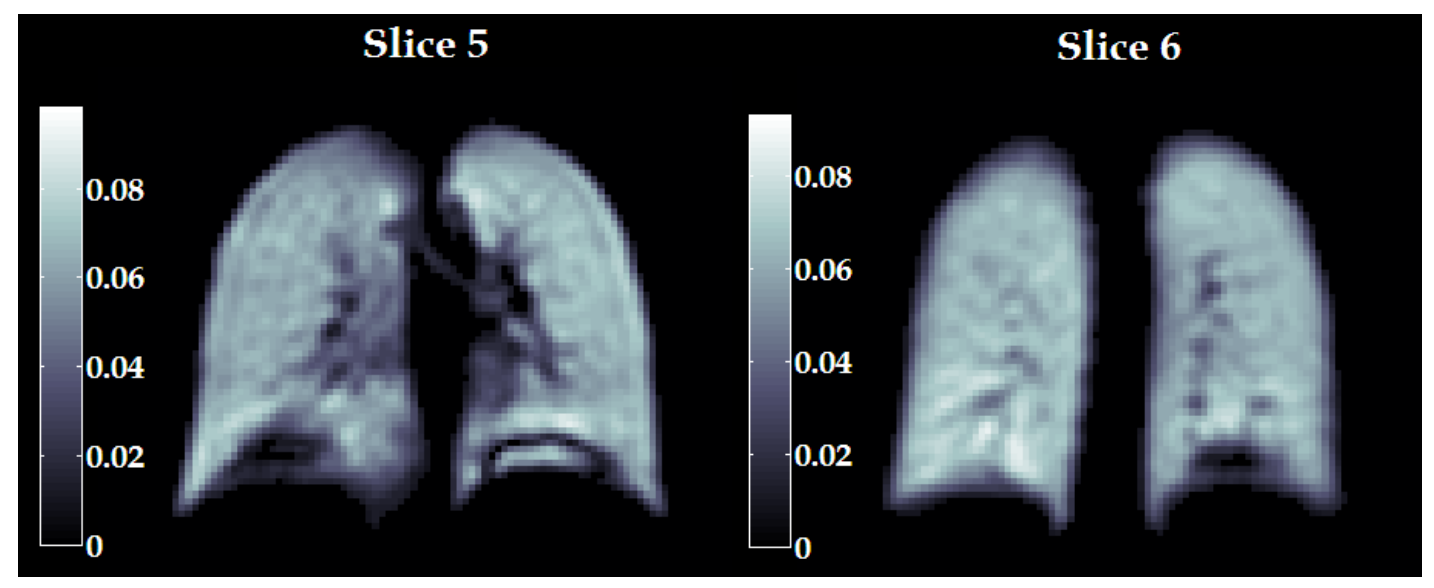

(a) Central Lung Image

(b) Posterior Lung Image

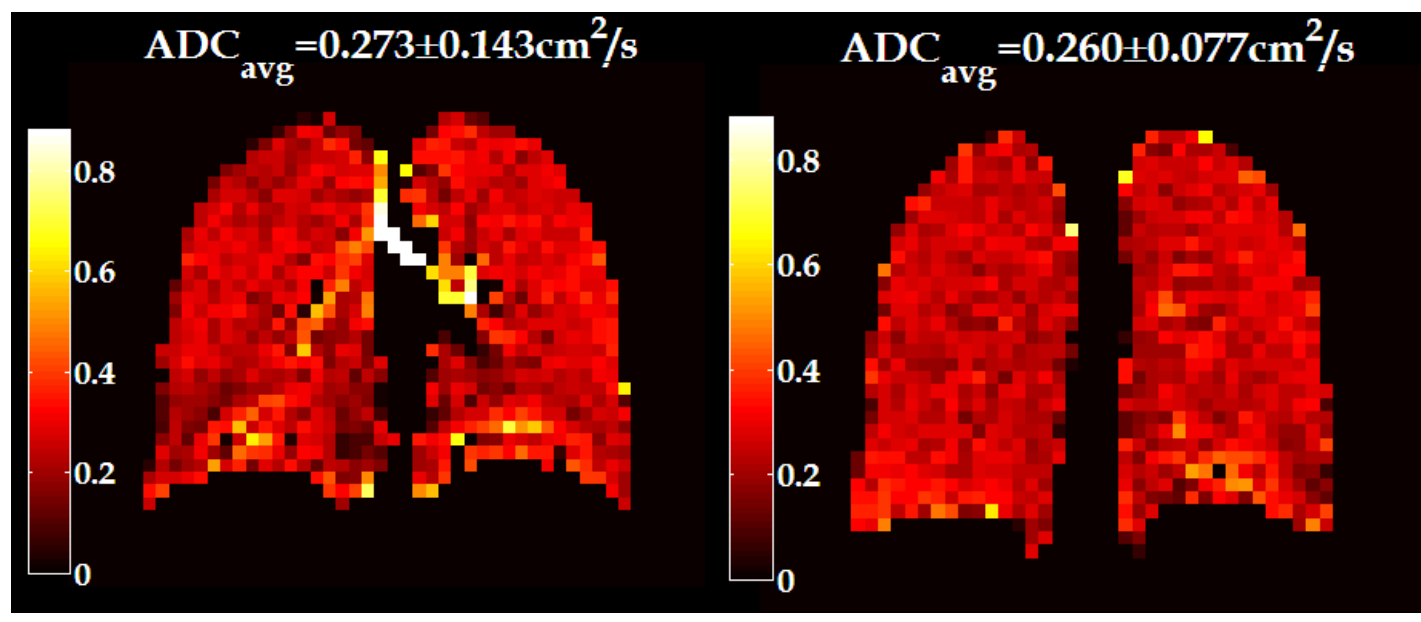

(c) Central Lung ADC Map

(d) Posterior Lung ADC Map

Figure 6.6: Raw ventilation images $\mathrm{I}(1)(\mathrm{a}-\mathrm{b})$ and ADC maps (c-d) from Before/After SSFP DWI in healthy subject 1226. The measured diffusivity is uniformly low in the bulk of the lung. ADC values close to the free diffusion coefficient of $D_{0} \approx 0.8 \mathrm{~cm}^{2} / \mathrm{s}$ are measured in the trachea and main bronchi. 
We also made diffusion-weighted measurements with the Before/After SSFP DWI pulse sequence described above in a volunteer who had been diagnosed with emphysematous Chronic Obstructive Pulmonary Disease (COPD) and a volunteer who was a heavy smoker but had not been diagnosed with COPD. Both subjects had reduced inspiratory capacity and were thus given gas mixtures with lower total volumes compared to the healthy subject: $300 \mathrm{~mL}$ of helium-3 and $400 \mathrm{~mL}$ of medical grade nitrogen for the COPD subject and $300 \mathrm{~mL}$ of helium-3 and $290 \mathrm{~mL}$ of medical grade nitrogen for the smoking subject. Ventilation images and ADC maps from a central lung slice and a posterior lung slice are shown for the COPD subject in Figure 6.7 and for the smoking subject in Figure 6.8.

The raw images of the COPD subject clearly show the regional nature of the airflow obstruction in COPD, as the lung was not uniformly ventilated with helium-3. The ADC values are elevated compared to the healthy subject, $A D C \approx 0.41 \mathrm{~cm}^{2} / \mathrm{s}$, and less uniformly distributed. In the smoking subject, the ventilation images also show areas of impaired ventilation (Figure 6.8a-b). However, the ADC values measured in the smoking subject appear very uniform and are only slightly elevated, ( $A D C \approx 0.31 \mathrm{~cm}^{2} / \mathrm{s}$ versus $A D C \approx 0.26 \mathrm{~cm}^{2} / \mathrm{s}$ in the healthy subject).

As described in Chapter 1, COPD is broadly categorized by two major characteristics: airway obstruction (chronic bronchitis) and alveolar tissue destruction (emphysema). Ventilation images alone can provide evidence of airway obstruction, by showing regions which do not receive helium-3 during inhalation. The ADC maps provide complementary information, by showing regional ADC differences indicative of airspace enlargement due to emphysematous tissue destruction. In the COPD subject, both of disease components are evident in our images. By contrast, the ventilation images from the smoking subject show evidence of airway obstruction consistent with their reduced inspiratory capacity, but the ADC 
maps suggest the absence of emphysema.

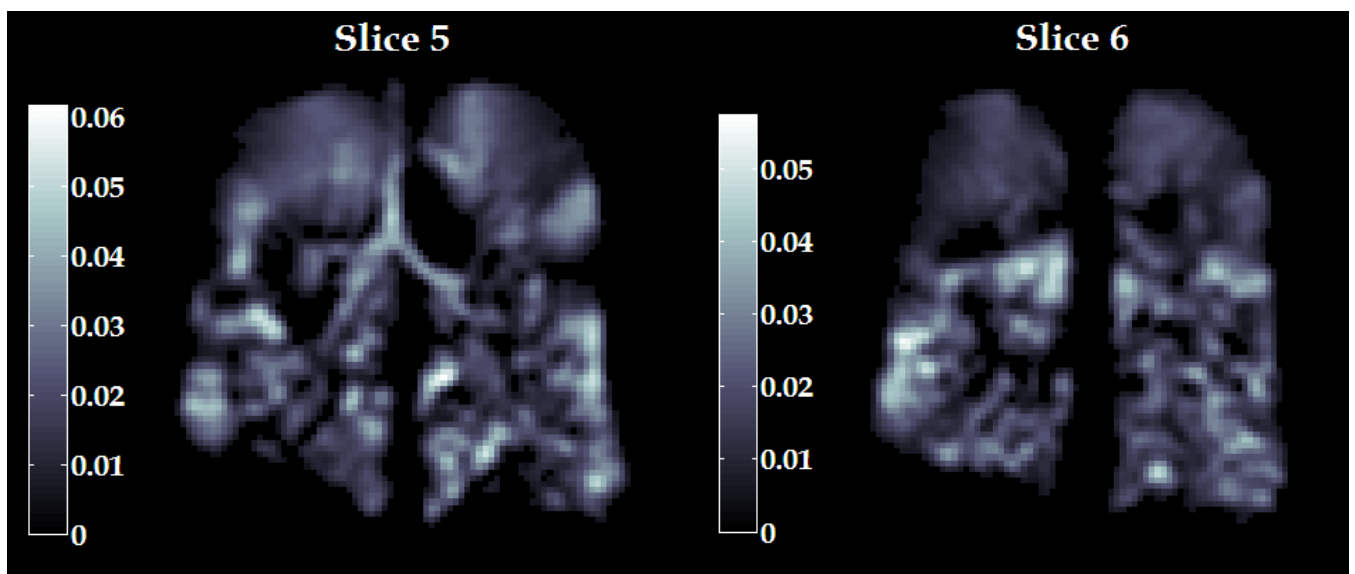

(a) Central Lung Image

(b) Posterior Lung Image

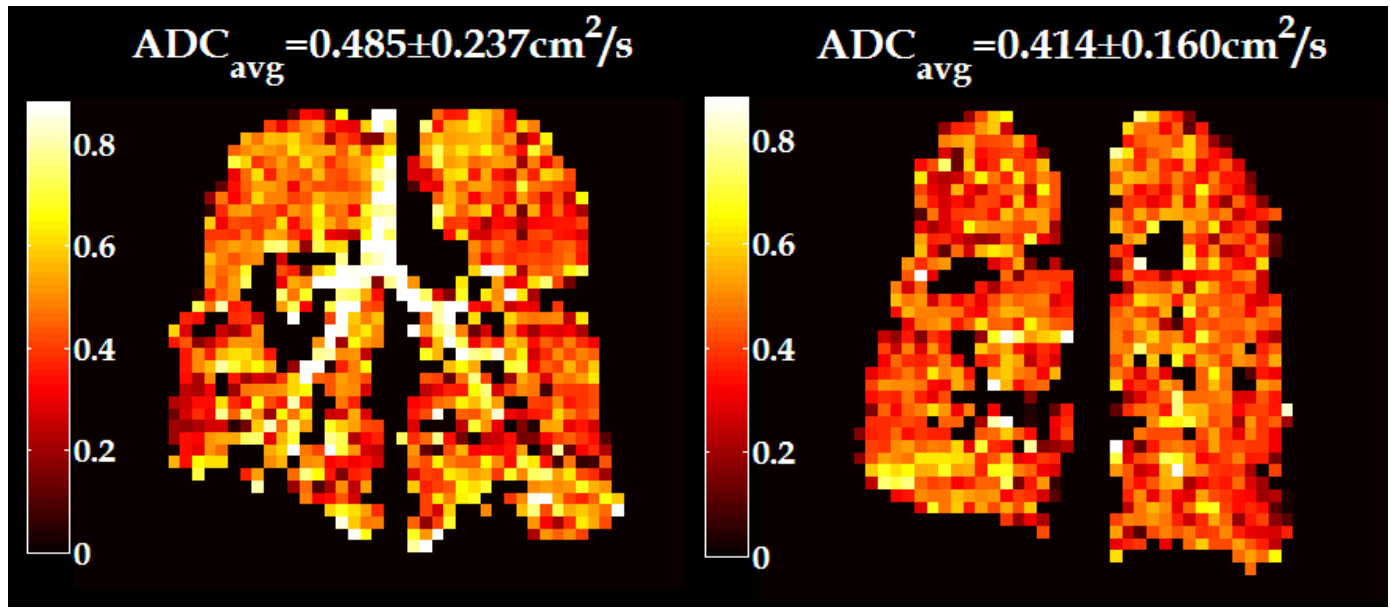

(c) Central Lung ADC Map

(d) Posterior Lung ADC Map

Figure 6.7: Raw ventilation images $\mathrm{I}(1)(\mathrm{a}-\mathrm{b})$ and ADC maps (c-d) from Before/After SSFP DWI in COPD subject 1238. The ventilation images show many areas which are poorly ventilated due to disease. The ADC maps reveal measured diffusivities that are elevated compared to the healthy subject and are nonuniformly distributed. 


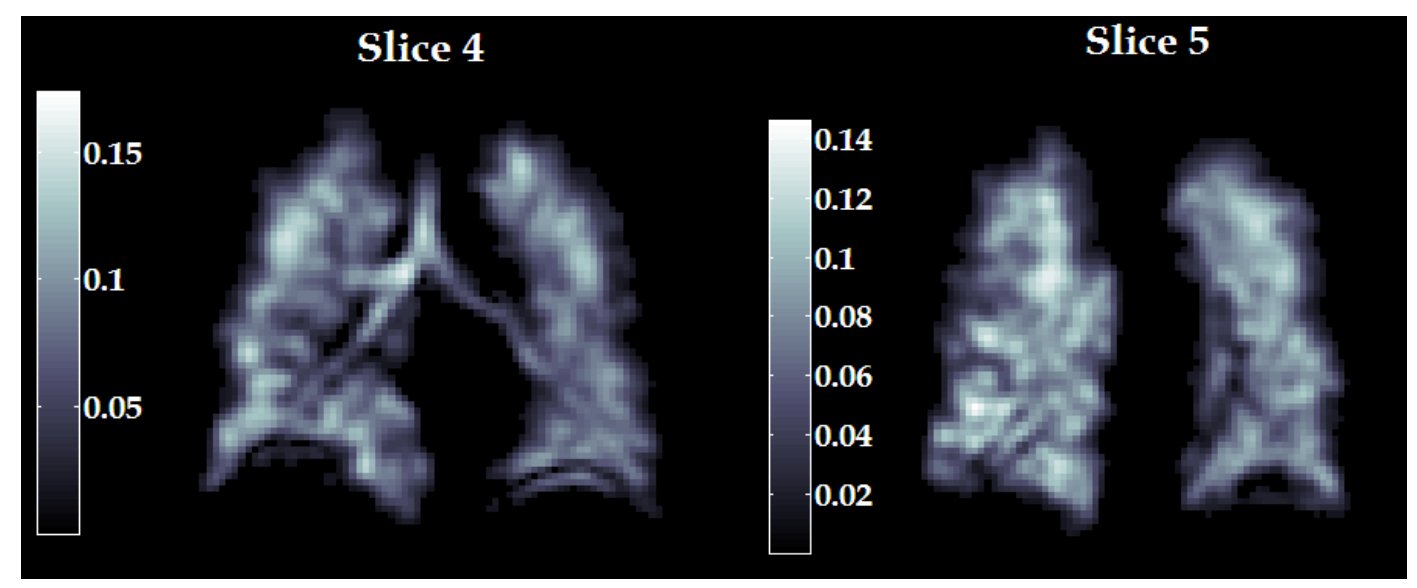

(a) Central Lung Image

(b) Posterior Lung Image

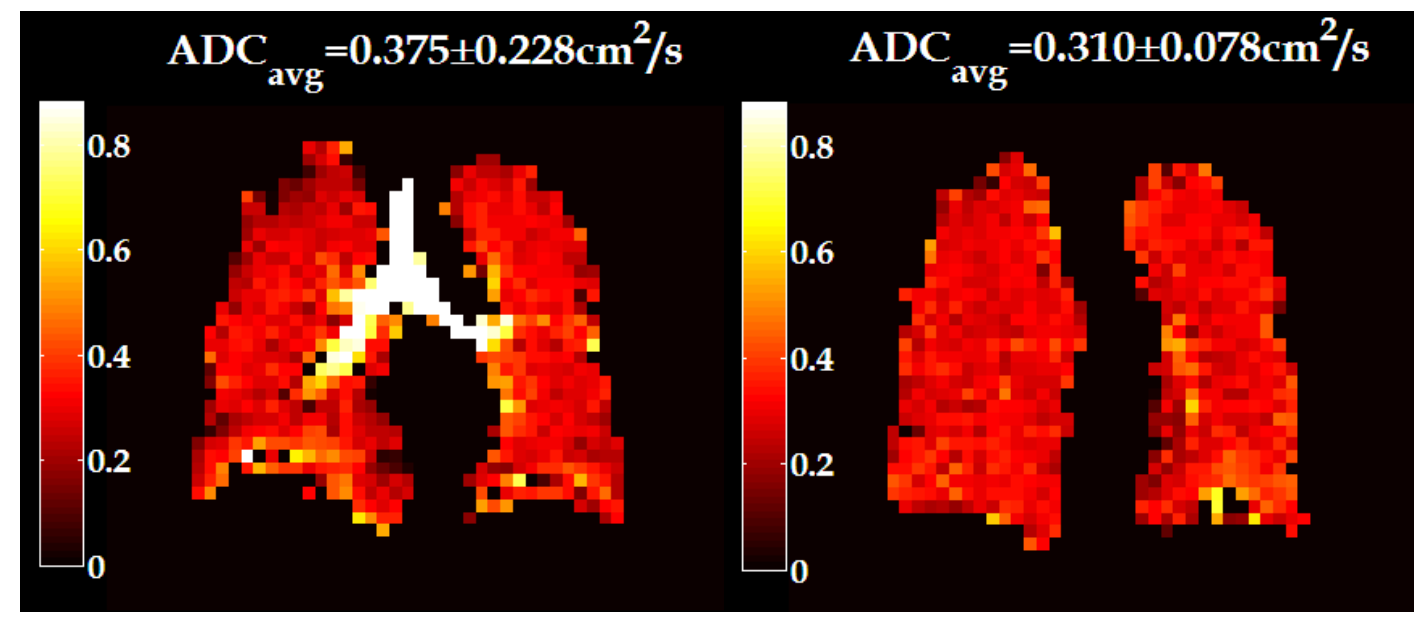

(c) Central Lung ADC Map

(d) Posterior Lung ADC Map

Figure 6.8: Raw ventilation images $\mathrm{I}(1)(\mathrm{a}-\mathrm{b})$ and ADC maps (c-d) from Before/After SSFP DWI in smoking subject 1233. The ventilation images show that many areas are poorly ventilated, similar to the COPD subject. However, the measured diffusivities are lower and much more uniform than the emphysematous COPD subject, and are only slightly higher than the healthy subject. 


\section{Chapter 7}

\section{Conclusions}

Diffusion-weighted magnetic resonance images of inhaled hyperpolarized gas provide a visualization of regional differences in lung microstructure by distinguishing between areas with restriction to gas movement and areas with freely moving gas. The measured diffusion coefficient increases in regions where the gas is less restricted. In the presence of emphysema, alveolar walls break down, creating larger diffusion spaces which can be detected using hyperpolarized gas diffusionweighted MRI. A key characteristic of diffusion in a restrictive environment is that the measured diffusivity depends on the time over which the measurement is made, in addition to the degree of restriction. Previous measurements of diffusivity in human lungs were focused in a range of diffusion times of $\Delta \sim 1-2 \mathrm{~ms}$, acquired using incoherent pulse sequences. The goal of this thesis was to develop a technique for making diffusivity measurements in the lung at shorter diffusion times.

The method we chose to explore was to incorporate diffusion-sensitization into Steady State Free Precession (SSFP) pulse sequences. SSFP pulse sequences can provide a greater signal-to-noise ratio than incoherent pulse sequences, due to the recycling of transverse magnetization from one excitation to the next. The 
higher SNR can benefit diffusion measurements made with short diffusion times $(\Delta<1 \mathrm{~ms})$, which have a reduced capacity to generate signal attenuation. A consequence of the coherent nature of SSFP pulse sequences is a sensitivity to offresonant magnetization. The effects of off-resonant magnetization can be minimized by using the shortest repetition time possible. Therefore, the SSFP technique is well suited to making diffusion measurements at short diffusion times.

In order to facilitate the development of a short time-scale diffusion pulse sequence, the first part of my work was the construction and commissioning of the Hybrid Helium-3 Polarizer. The Hybrid Polarizer provides a supply of highly polarized helium-3 at nearly 1.5 times the polarization $\left(P_{H e 3} \sim 60 \%\right.$ versus $\left.\sim 40 \%\right)$ in more than double the batch size (3 L versus $1.3 \mathrm{~L})$ than any SEOP-based helium3 polarizer used for medical imaging in the published literature. This significantly higher polarization means that the same image SNR can be obtained using a smaller helium-3 dose per scan. The more efficient use of helium-3 for medical imaging is important due to the current scarcity of helium-3, illustrated in Figure 7.1 .

For thermally polarized magnetization, it is well known that the application of an SSFP pulse sequence leads to a steady state in which the transverse magnetization level after every pulse is constant. By simulating evolution of the magnetization vector under the influence of an SSFP pulse sequence, we were able to determine that a different steady state is reached for hyperpolarized magnetization in which the signal declines from one excitation to the next, but the ratio between consecutive pulses is constant:

$$
\frac{M(n+1)}{M(n)}=\frac{M(n+2)}{M(n+1)}
$$

We used this result to derive a closed-form expression for this equilibrium ratio 


\section{Demand for ${ }^{3} \mathrm{He}$ accelerated after $9 / 11$, drawing down US stocks}

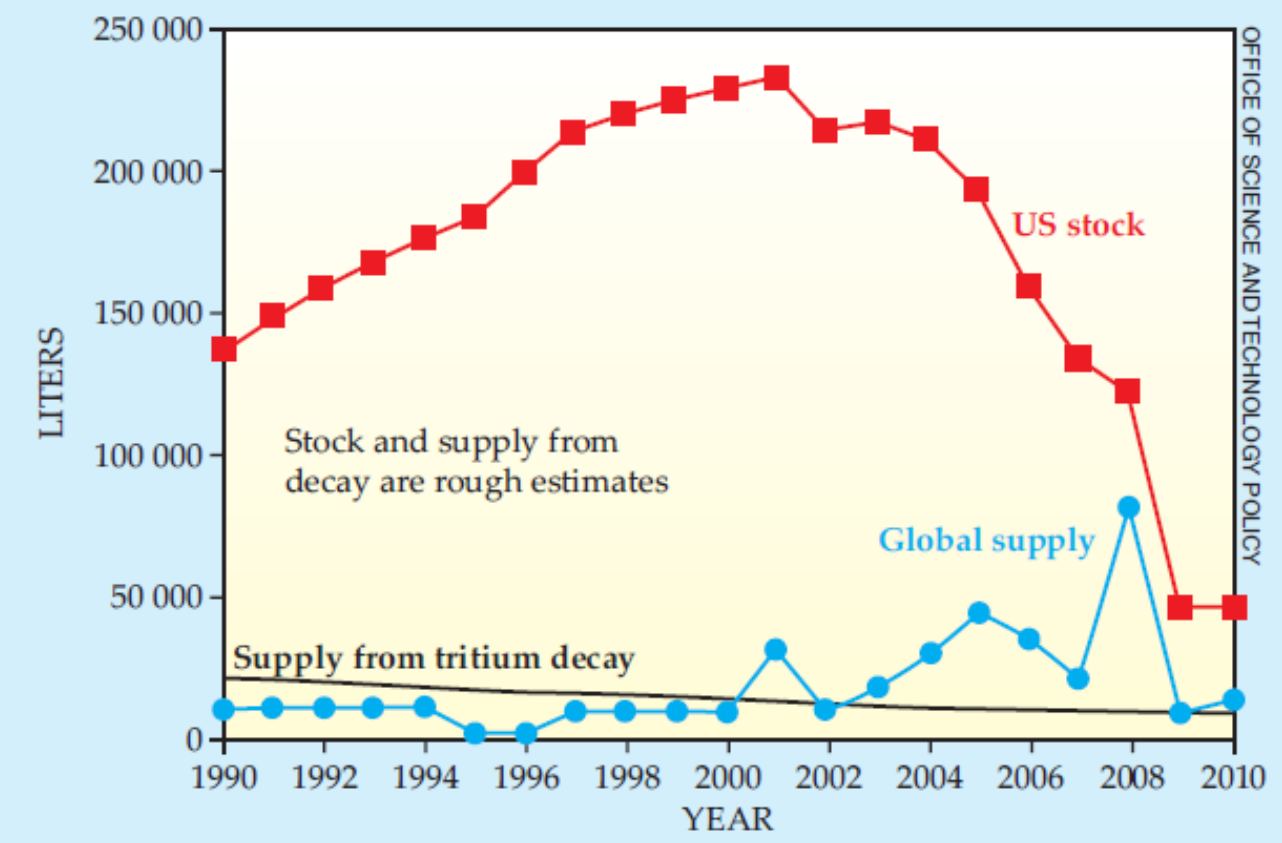

Surging demand from radiation portal monitors and plunging nuclear weapons requirements combined to deplete US stocks of helium-3 by more than threequarters from their peak in 2001. DOE suspended all releases of ${ }^{3} \mathrm{He}$ last year. The agency has allocated just 12000 liters for 2010, which compares to an estimated annual demand upward of 70000 liters.

Figure 7.1: The declining stock of US helium-3 reserves [41].

for the case of on-resonance magnetization, and used numerical simulations to study how it changes in the presence of off-resonance magnetization. The ratio depends on pulse sequence parameters such as flip angle and repetition time, so there is the potential that our result can be used to optimize SSFP pulse sequences by manipulating these parameters.

We developed several methods of inducing diffusion-weighted contrast in the steady state free precession sequence: the Symmetric, Asymmetric, Group of Three and Before/After diffusion-weighted SSFP pulse sequences. We explored the influence of off-resonant spins on each of these pulse sequences and were able to identify the most promising candidates for measuring diffusion attenuation. The 
Asymmetric and Group of Three techniques employ a variable signal attenuation between different TR periods, which results in a steady state signal that alternates between two or more signal levels while declining towards thermal equilibrium. There are interesting and potentially useful features which come out of this behavior such as the enhanced diffusion contrast in the Asymmetric method, however these pulse sequences are particularly sensitive to the effects of off-resonance magnetization, and thus not ideal for use in the lung. Our results showed that the Before/After method of introducing diffusion attenuation was the most insensitive to off-resonance magnetization, and therefore was the most promising option for measuring restricted diffusion in porous environments such as the lung.

We acquired time-dependent diffusivity measurements in glass-bead phantoms using a global Before/After diffusion-weighted SSFP pulse sequence over a range of $\Delta=300-800 \mu \mathrm{s}$. We fit these measurements to the theoretical time dependence of $D(t)$ in the region between the linear short-time scale and the constant long-time behavior in order to extract a measurement of the porosity $\phi$ of the bead packs, which is a quantifiable structural parameter. The surface-to-volume ratio of the pore space is another quantifiable structural parameter that can be extracted from diffusivity measurements, as the slope of the short time-scale linear region of the time-dependent diffusivity curve is to proportional to $S / V$. We determined that for packs of spherical beads, the range of diffusion times at which the time dependence of the diffusivity is dominated by the linear behavior is:

$$
t \leq \frac{t_{0}}{4}=\frac{1}{4(S / V)^{2} D_{0}} .
$$

We were not able to reach this limit using helium-3 in our bead phantoms, however if measurements were made with xenon gas $\left(D_{0}=0.06 \mathrm{~cm}^{2} / \mathrm{s}\right)$, the linear region would be expected to extend to more accessible diffusion times even in the 
most restrictive $1 \mathrm{~mm}$-bead phantom, $\frac{t_{0}}{4}=470 \mu \mathrm{s}$.

We also acquired time-dependent diffusivity measurements in human subjects using the same Before/After diffusion-weighted SSFP pulse sequence over the same range of $\Delta=300-800 \mu \mathrm{s}$. These measurements suggested that the timedependence of the diffusivity in human lungs exhibits similar behavior to that observed in the bead phantoms. The shape of the time-dependent diffusivity curve was markedly different for the subject with COPD compared to the healthy subjects.

Figure 7.2a graphs the theoretical time dependence of diffusivity for our three glass-bead phantoms. In Figure $7.2 \mathrm{~b}$, the relative difference between the $1 \mathrm{~mm}$ bead phantom curve and both the $2 \mathrm{~mm}$ and $3 \mathrm{~mm}$ curves are plotted versus diffusion time, revealing a peak near $\Delta \sim 400 \mu \mathrm{s}$. The shape of this relative difference curve indicates that diffusion measurements made at the peak time would be maximally sensitive to changes in diffusion restriction. Global time-dependent diffusivity measurements in human subjects could be used to generate such a plot for lung diffusivity, which would then serve as a guide for the optimal diffusion time at which to make ADC maps.

We made ADC maps in volunteer subjects using a diffusion time of $\Delta=500 \mu \mathrm{s}$, which is a much shorter diffusion time than previously reported in humans. These initial results clearly reveal differences in restricted diffusion between the healthy subject and the subject with emphysema. Now that basic sensitivity has been demonstrated, further optimization of the Before/After SSFP DWI pulse sequence parameters could include working to increase imaging resolution and to decrease repetition time.

In both imaging pulse sequences we developed (the Before/After and the Group of Three SSFP DWI), the presence of off-resonant magnetization affected the ability 


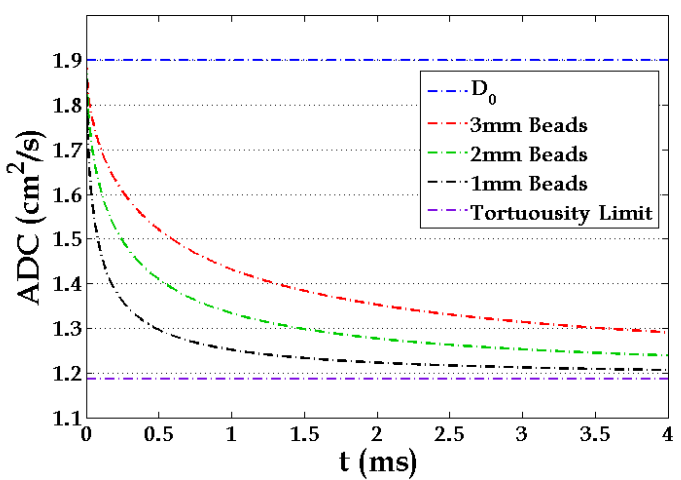

(a) Diffusivity

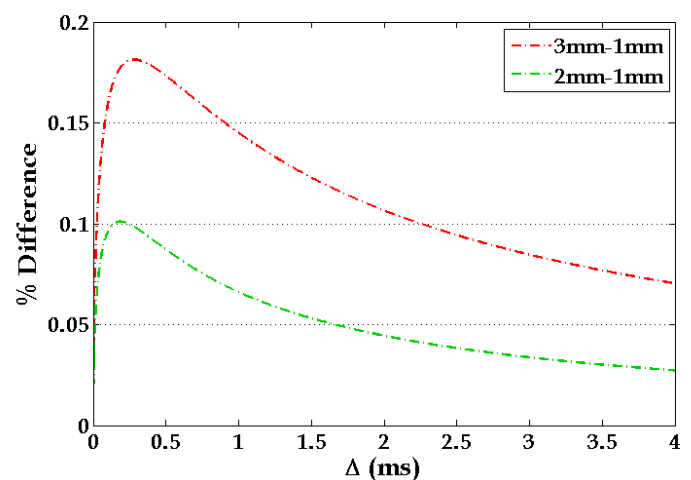

(b) Relative Difference

Figure 7.2: (a) Calculated diffusivity versus diffusion time for several degrees of restriction in glass beads. (b) Relative difference between the $1 \mathrm{~mm}$-bead phantom curve and the $2 \mathrm{~mm}$ and $3 \mathrm{~mm}$ curves from (a) show a peak sensisivity near $\Delta \sim$ $400 \mu \mathrm{s}$.

of the sequence to produce accurate ADC measurements. When using the Group of Three SSFP DWI pulse sequence, we demonstrated that off-resonant precession of $\beta>60^{\circ}$ per TR resulted in signal ratios with the opposite contrast to the applied diffusion weighting. In images produced with the Before/After SSFP DWI pulse sequence, regions with off-resonant precession approaching $\beta=180^{\circ}$ per TR had greatly reduced signal, which appeared to distort the calculated diffusivity. Both of these undesired effects could be reduced by shortening $T R$.

The precession frequency, and thus the off-resonant phase accumulation for a given repetition time, is determined by both the magnetic field and the gyromagnetic ratio of the particle. Xenon-129 has a lower gyromagnetic ratio than helium-3, so for the same degree of magnetic field inhomogeneity, xenon-129 will accumulate a smaller phase difference than helium-3 over the same TR. Thus, diffusionweighted SSFP images made with hyperpolarized xenon-129 have the potential to be less susceptible to off-resonance effects caused by magnetic field inhomogeneities.

Reducing the size of magnetic field inhomogeneities is another strategy for re- 
ducing off-resonance magnetization effects. In the $1.5 \mathrm{~T}$ scanner used in this work, our Group of Three imaging results indicated that the field inhomogeneities exceeded $\sim 2.3 \mathrm{ppm}$ in some regions of the lung. An MR scanner with a holding field of $B_{0}=0.75 \mathrm{~T}$ with the same relative inhomogeneity would have half of the absolute field difference. The smaller changes in field should result in less phase accumulation during the same $T R$ period.

The combination of efficient use of hyperpolarized magnetization with an SSFP pulse sequence and high initial helium-3 polarization from the Hybrid Polarizer has recently been used to generate high-quality helium-3 ventilation images, shown in Figure 7.3, while reducing the dose of helium-3 from the standard of 300-400 mL to only $100 \mathrm{~mL}$. This remarkable result is a strong indication of the potential that exists to improve hyperpolarized gas MR by incorporating new techniques from both the MR and the nuclear physics communities. 


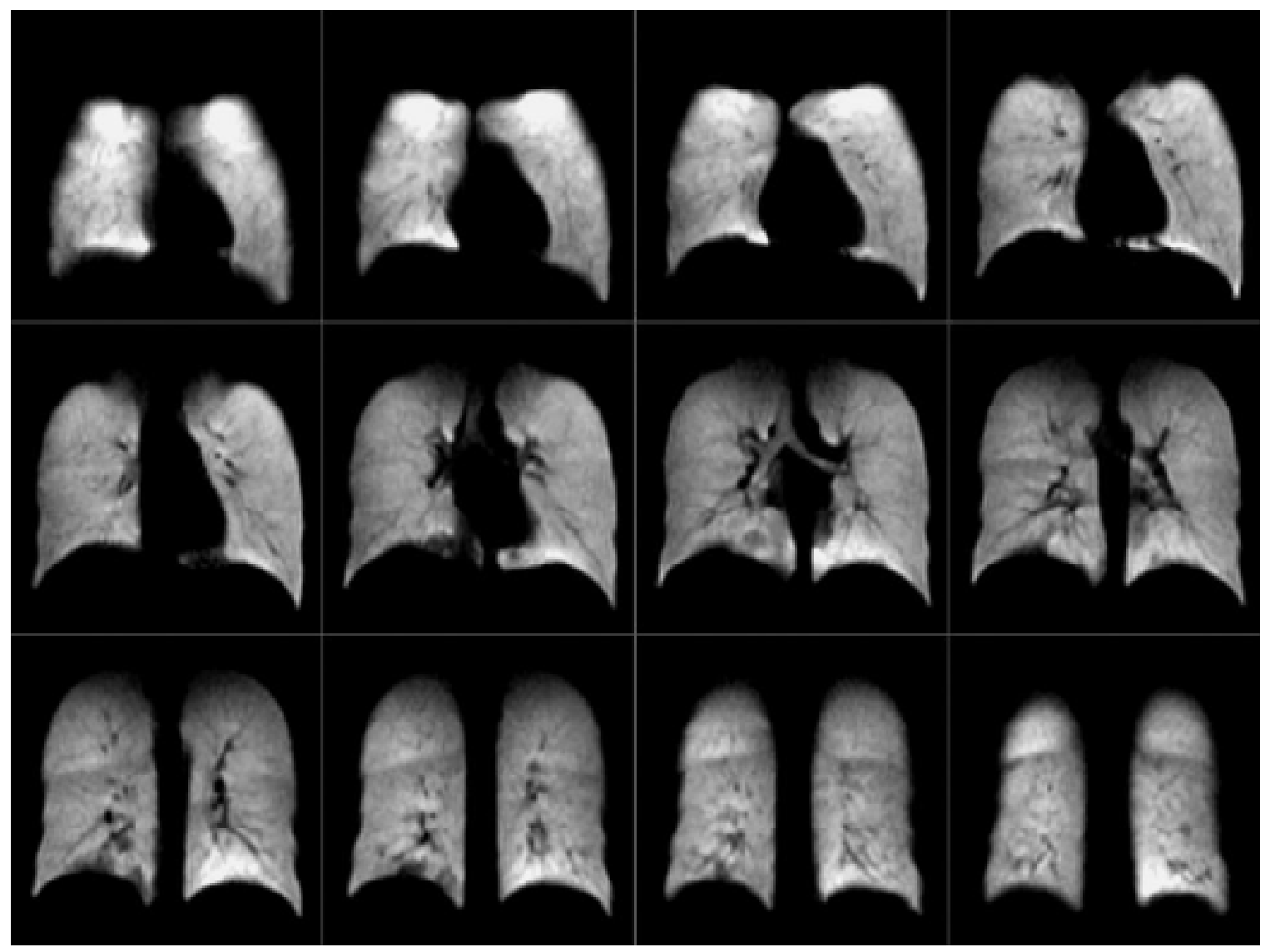

Figure 7.3: Ventilation images acquired using $100 \mathrm{~mL}$ of ${ }^{3} \mathrm{He}$ from the Hybrid Polarizer (and supplemental nitrogen to increase total inhaled volume) using an SSFP pulse sequence. In plane resolution is $3.3 \times 3.3 \mathrm{~mm}$ with $10 \mathrm{~mm}$ thick slices. The SNR of these images is comparable to that is images made with 3-4 times more helium-3 using incoherent imaging and lower gas polarization [83]. 


\section{Appendix A}

\section{Hybrid Polarizer Limitations: Masing and the X-Factor}

Two important components limit the performance of the new hybrid polarizer, masing and each cell's X-factor. These concepts are important to consider when trying to optimize the helium-3 polarization.

\section{A.1 Masing}

Masing is a term used to describe the behavior which limits the maximum polarization a substance can achieve while being pumped to the high energy state, in which the magnetic field due to the particles is antiparallel to the main alignment field. As the polarization reaches a threshold level, spontaneous spin flips increase in probability. Each spontaneous flip from the high state to the low state releases an unpolarized photon, which is then able to stimulate spin flips in other particles. The system can not maintain a polarization above this threshold in the high energy state. However, if a system which mases is polarized by pumping the low energy state, the maximum polarization is not affected by the masing threshold as sponta- 
neous spin flips are far less frequent in the low energy state. This phenomenon has been observed at the SEOP setup in the physics building and several JLab experiments [58], and was described in [84] as being attributed to a coupling between the helium-3 magnetization and a set of transverse coils. Tests in the physics lab have not shown this link, as the behavior seems constant even as the distances between the cell and coils are changed.

During the first test of the hybrid polarizer we were unsure to which of the energy states we were pumping the helium-3 nuclei, so we were prepared for the possibility that our cell might mase. After several hours of pumping, the polarization signal exhibited a characteristic of masing, the abrupt flat top seen in Figure A.1a. We varied the NMR amplitude towards the end of this test to ensure that we had sufficient signal to measure, which caused the rise and subsequent decline in signal as the measurements were made. At this point we suspected the cell was masing, so we shut off the lasers and rotated the quarterwave plates by $90^{\circ}$. This step reverses the circular polarization of the light, which changes the state that is being pumped. The result is the data shown in Figure A.1b, which has the expected exponential signal increase over time.

This test confirmed that the cell was masing. To reduce this effect, we have incorporated a pair of counter-wound gradient coils around the main holding field coils. The coils consist of 9 turns each of XX gauge copper wire, and are secured to the holding field coils with Kapton high temperature resistant tape (see Figure A.2). The coils are powered by a $X X X_{v} X A m p$ power supply. The strength of the field provided by the gradient coils needs to balance the priorities of being strong enough to create a transition frequency gradient to reduce the propagation of spin flips with being small enough to not contribute to large polarization during AFP measurements. We estimate that this field should be in the range of $10 \mathrm{mG} / \mathrm{cm}$. 


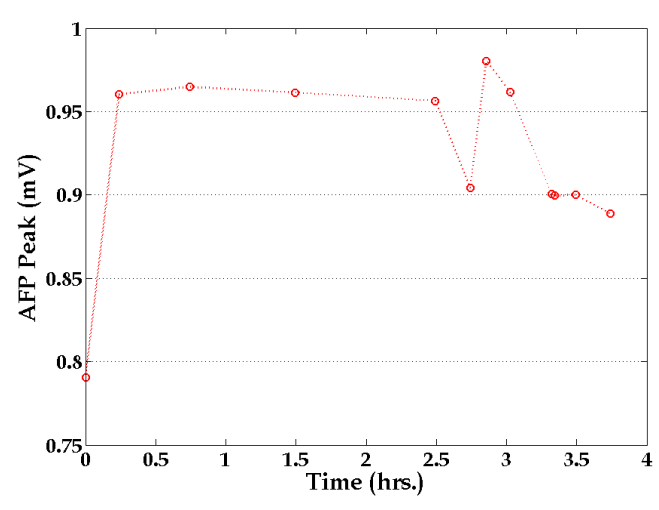

(a) Pumping the HI state

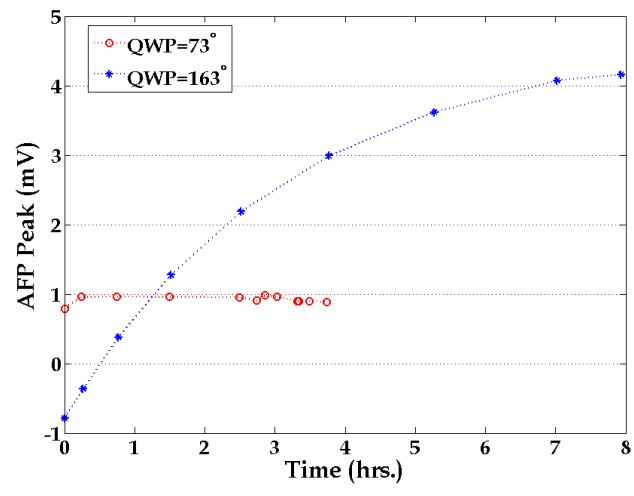

(b) Pumping the LO state

Figure A.1: The NMR signal measured from the first attempts at using the hybrid polarizer. The quarterwave plates were rotated $90^{\circ}$ between runs, which reverses the circular polarization and changes the state being pumped. The cell mases in (a), plateauing at a relatively low signal level. When the low energy state is pumped (b), the polarization increases with the expected exponential dependence.

For our first test we varied the gradient coil current and monitored the AFP losses while pumping the low energy state, selecting the current which minimized the $\operatorname{loss}\left(I_{G}=2.5 A\right)$.

For a more thorough test of the masing effect, we arranged for our system to pump the high energy state by reversing the holding field direction. Once the polarizer was put into commission, this method of changing the pump state is preferred over adjusting the quarterwave plate angle, as imprecise settings can reduce the degree of circular polarization of the pump light. We began pumping the cell with the gradient coil current set to $I_{G}=2.5 \mathrm{~A}$. With these settings the cell was still able to mase, although the threshold signal level increased compared to the original test with no gradient coil. After it was clear the cell had reached the masing threshold, we increased the gradient coil current by $0.5 \mathrm{~A}$. The results of this test are shown in Figure A.3. For each value of th coil current, the saturation signal is higher than the previous signal until the gradient current reaches $I_{G}=$ 5.0A. At this point, the losses between NMR points increases and the saturation signal is reduced. 


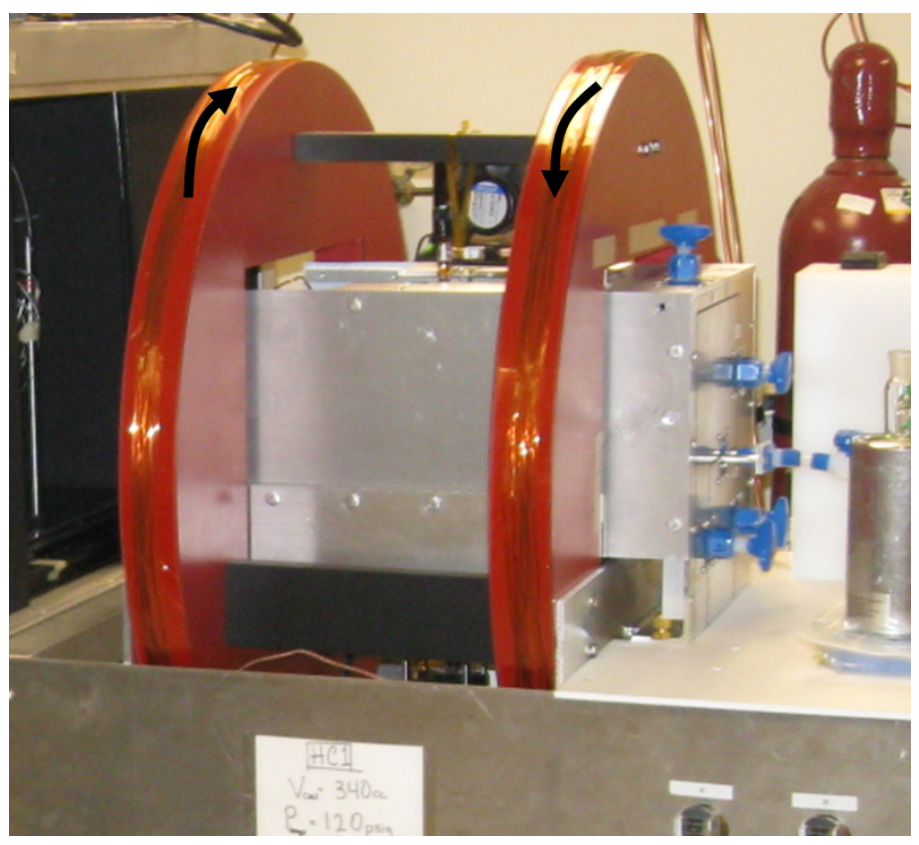

Figure A.2: The gradient coils installed on the hybrid polarizer. Their are 9 turns of copper wire in each coil, with black arrows indicating the direction of current flow.

In order to minimize the effects of masing, the hybrid polarizer is routinely operated in the low energy pumping state with the gradient coil current set to $I_{G}=2.5 \mathrm{~A}$. We chose this value due to our experiences with large AFP losses described in Section 3.6. When pumping to the low energy state, masing occurs during the AFP measurement when the spins are flipped if they are polarized above the masing threshold. This means the AFP loss per measurement increases as the polarization of the helium-3 nuclei increases. We chose the gradient coil current that balanced this AFP loss at high polarizations with the baseline AFP loss at below threshold polarizations, which are larger when the gradient coil current is increased. 


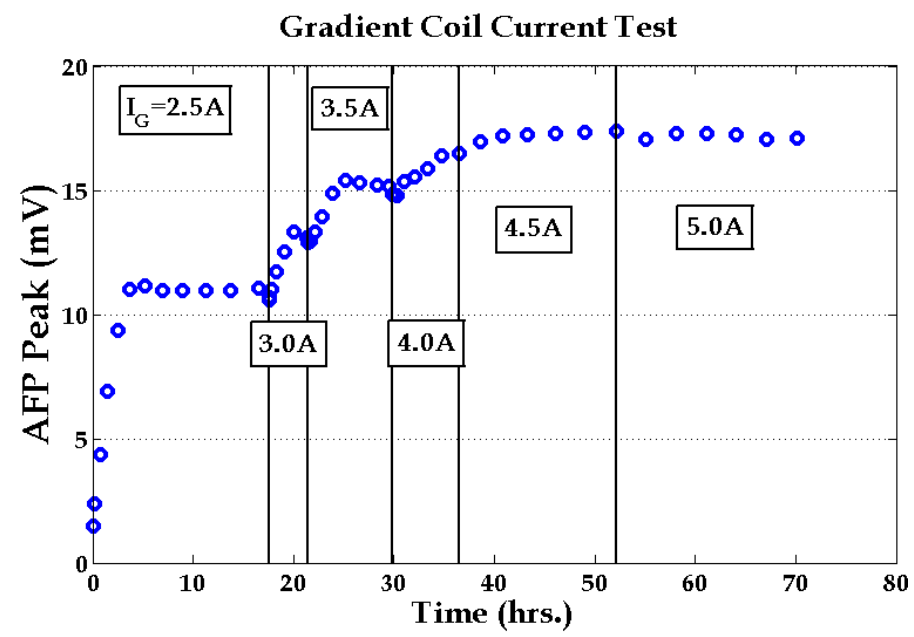

Figure A.3: NMR signal amplitude as the gradient coil current is increased. The signal was considered to have reached a threshold when the NMR signal remained constant or decreased from one point to the next. The current setting with the highest saturation signal was $I_{G}=4.5 \mathrm{~A}$.

\section{A.2 X-Factor}

Babcock et al have suggested an alteration to the achievable helium-3 saturation polarization given in equation 3.5 [85]. There exists an additional surface-relaxation mechanism which scales with cell surface-to-volume ratio and alkali metal density. The additional relaxation reduces the helium-3 saturation polarization

$$
P_{\text {HeSat }}=P_{R b} * \frac{\gamma_{s e}}{\gamma_{s e}(1+X)+\Gamma_{s d}}
$$

where the $\mathrm{X}$ factor accounts for the additional relaxation and appears to scale with surface-to-volume ratio and alkali density. When determining the quality of a polarization cell, it is useful to determine the $\mathrm{X}$ factor for that cell. Having the $\mathrm{X}$ factor allows for a better judgment on how close the hybrid polarizer's set up is to optimal for that particular cell. 
In solving for $X$ we make an approximation for the spin-exchange rate

$$
\gamma_{s e}=\frac{\Gamma_{u p}-\Gamma_{s d}}{1+X}
$$

where $\Gamma_{u p}=1 / T_{u p}$, the spinup time constant. We also replace $P_{R b}$ with $P_{A}$ for average alkali metal polarization due to the addition of potassium in the hybrid polarizer.

$$
X=\frac{P_{A}}{P_{\text {HeSat }}}\left(1-\frac{\Gamma_{s d}}{\Gamma_{u p}}\right)-1
$$

Each of the parameters in equation A.3 can be determined from either a spinup or a spindown except the alkali polarization. The hybrid polarizer lacks the diagnostic tools used in other labs to measure alkali polarization. We calculate $X$ with $P_{A}=1$ and set an upper bound on the $X$ factor for the cell based on the saturation polarization and the spinup and spindown time constants.

Table A. 1 lists the $\mathrm{X}$ factor values calculated whenever the hybrid polarizer had a paired spinup and spindown performed with the same gas mixture. The average $X$ factor measured in $\mathrm{HC} 1 \mathrm{X}_{\mathrm{Avg}}(\mathrm{HC} 1)=0.44$. For $\mathrm{HC}$, the first measurement of $X=0.27$ may have been influenced by a large cloudy substance covering the alkali metal pool which accumulated while the cell was in storage. 


\begin{tabular}{|c|c|c|c|c|c|c|}
\hline Cell & Date & Pressure $(\mathrm{psig})$ & $\Gamma_{u p}(1 / \mathrm{hrs})$ & $P_{\text {HeSat }}$ & $\Gamma_{s d}(1 / \mathrm{hrs})$ & X Factor \\
\hline HC1 & $05 / 26 / 2008$ & 120 & $1 / 3.54$ & 65.4 & $1 / 49.2$ & 0.42 \\
\hline & $06 / 02 / 2008$ & 120 & $1 / 4.86$ & 59.6 & $1 / 47.2$ & 0.51 \\
\hline & $11 / 14 / 2008$ & 120 & $1 / 3.68$ & 59.4 & $1 / 37.1$ & 0.52 \\
\hline & $08 / 10 / 2009$ & 120 & $1 / 3.81$ & 63.6 & $1 / 35.6$ & 0.40 \\
\hline & $08 / 14 / 2009$ & 41.4 & $1 / 3.85$ & 65.4 & $1 / 44.2$ & 0.40 \\
\hline & $08 / 09 / 2010$ & 120 & $1 / 3.86$ & 66.0 & $1 / 39.4$ & 0.37 \\
\hline & $02 / 13 / 2011$ & 110 & $1 / 3.54$ & 60.0 & $1 / 33.4$ & 0.49 \\
\hline \hline HC2 & $06 / 28 / 2011$ & 120 & $1 / 4.8$ & 62.2 & $1 / 22.8$ & 0.27 \\
\hline & $07 / 03 / 2011$ & 120 & $1 / 4.0$ & 60.9 & $1 / 27.8$ & 0.41 \\
\hline Cell & Date & Pressure $(\mathrm{psig})$ & $\Gamma_{u p}(1 / \mathrm{hrs})$ & $P_{\text {HeSat }}$ & $\Gamma_{s d}(1 / \mathrm{hrs})$ & X Factor \\
\hline
\end{tabular}

Table A.1: Spinup and spindown parameters for $\mathrm{HC} 1$ and $\mathrm{HC} 2$ and the calculated $X$ factors. 


\section{Appendix B}

\section{Hybrid Polarizer Components}

The following section provides information about specific components which were used in the construction and operation of the Hybrid Polarizer.

\section{B.1 Gas System Details}

The gas system is divided into two sections, the upper and the lower gas systems. The lower system is made of stainless steel tubing and has VCR connections and valves. It contains the ${ }^{3} \mathrm{He}$ Bottle, the vacuum pump, and the UHP Nitrogen. The upper system is made of aluminum tubing and has primarily Swagelok connections and valves. 


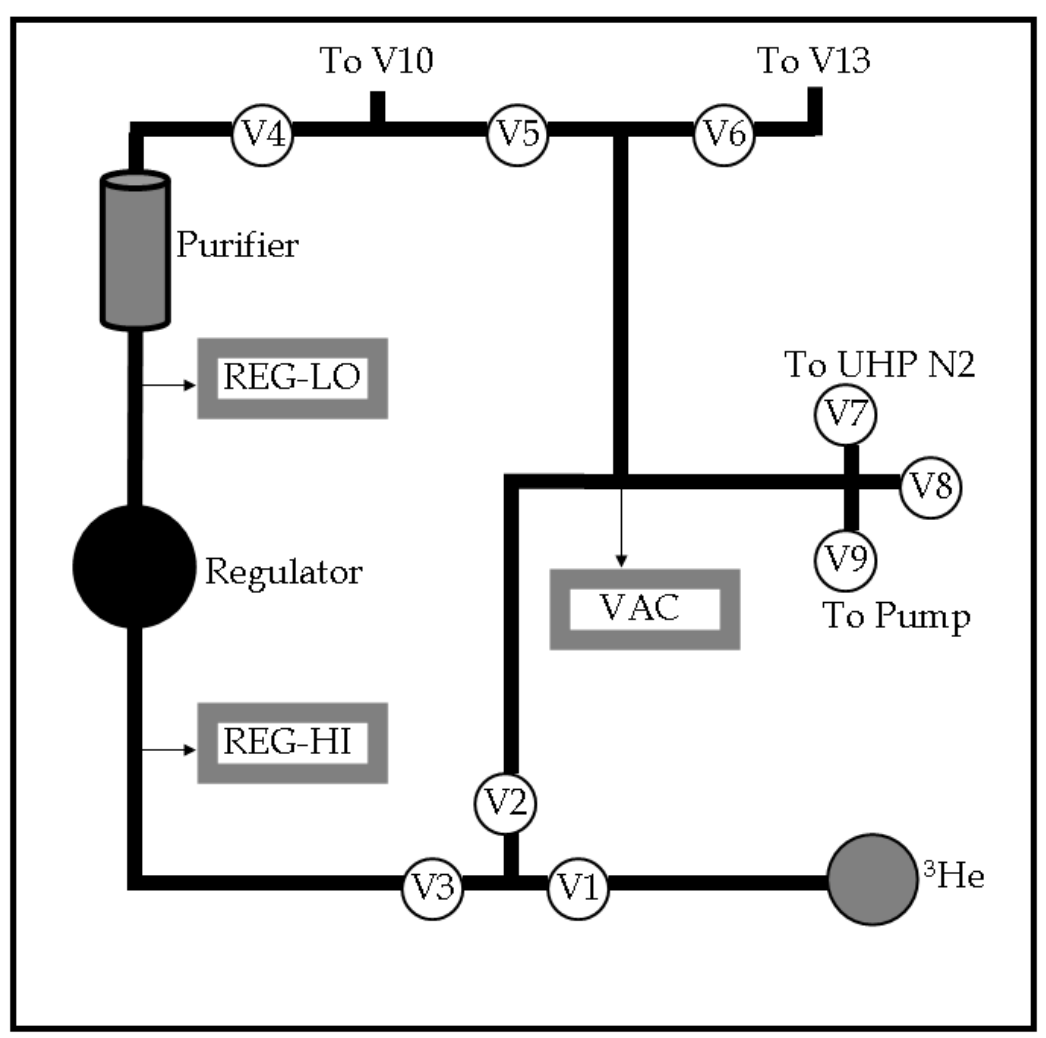

Figure B.1: Lower Gas System 


\begin{tabular}{|c|c|}
\hline Item & Description \\
\hline${ }^{3}$ He Bottle & $\begin{array}{l}\text { High pressure bottle containing } \sim 53 \mathrm{~L} \text { of helium. Pressure usu- } \\
\text { ally ranges from } 1800 \mathrm{psi} \text { to } 100 \mathrm{psi} \text {. Serial number of the bottle } \\
\text { must be noted on every batch dispensed and should be recorded } \\
\text { in the logbook when a bottle is attached or removed. }\end{array}$ \\
\hline V1 & $\begin{array}{l}\text { Valve 1- isolates the line to the bottle from the rest of the gas } \\
\text { system. }\end{array}$ \\
\hline V2 & $\begin{array}{l}\text { Valve } 2 \text { - connects the Vacuum Manifold to the Regulator HI and } \\
\text { the bottle. }\end{array}$ \\
\hline V3 & $\begin{array}{l}\text { Valve 3- isolates the Regulator HI from the rest of the system } \\
\text { when the regulator is closed. }\end{array}$ \\
\hline REG-HI & $\begin{array}{l}\text { A transducer that displays the pressure on the high side of the } \\
\text { regulator, which is also the }{ }^{3} \mathrm{He} \text { Bottle pressure. (psia) }\end{array}$ \\
\hline Regulator & Tescom Regulator: $\mathrm{IN}_{M A X}=3500 p s i$, OUT $_{M A X}=150 p s i$ \\
\hline REG-LO & $\begin{array}{l}\text { A transducer that displays the pressure on the low side of the } \\
\text { regulator, which is also the cell pressure. (psig) }\end{array}$ \\
\hline $\mathrm{V} 4$ & $\begin{array}{l}\text { Valve } 4 \text { - connects the Regulator LO to V10, which leads to the } \\
\text { cell. }\end{array}$ \\
\hline V5 & $\begin{array}{l}\text { Valve 5- connects the Vacuum Manifold to the Regulator LO and } \\
\text { V10, which leads to the cell. }\end{array}$ \\
\hline V6 & $\begin{array}{l}\text { Valve 6- connects the Vacuum Manifold to V13, which leads to } \\
\text { the outlet. }\end{array}$ \\
\hline V7 & $\begin{array}{l}\text { Valve 7- connects to the Ultra High Purity (UHP) Nitrogen, } \\
\text { which is used for purging the gas system. }\end{array}$ \\
\hline V8 & $\begin{array}{l}\text { Valve 8- outlets to the room. Used for setting the } \mathrm{UHP}_{2} \text { flow to } \\
\text { an acceptable level. }\end{array}$ \\
\hline V9 & Valve 9- connects to the vacuum pump. \\
\hline VAC & Vacuum Pressure Gauge $(m T)$ \\
\hline V10 & $\begin{array}{l}\text { Valve 10- connects the cell to the lower gas system, specifically } \\
\text { the Regulator LO side. }\end{array}$ \\
\hline V11 & Valve 11- connects the cell to the outlet. \\
\hline V12 & $\begin{array}{l}\text { Valve 12- connects the outlet to the Medical Grade (med) Nitro- } \\
\text { gen. }\end{array}$ \\
\hline V13 & $\begin{array}{l}\text { Valve 13- connects the outlet to the lower gas system, specifically } \\
\text { the vacuum pump. }\end{array}$ \\
\hline
\end{tabular}




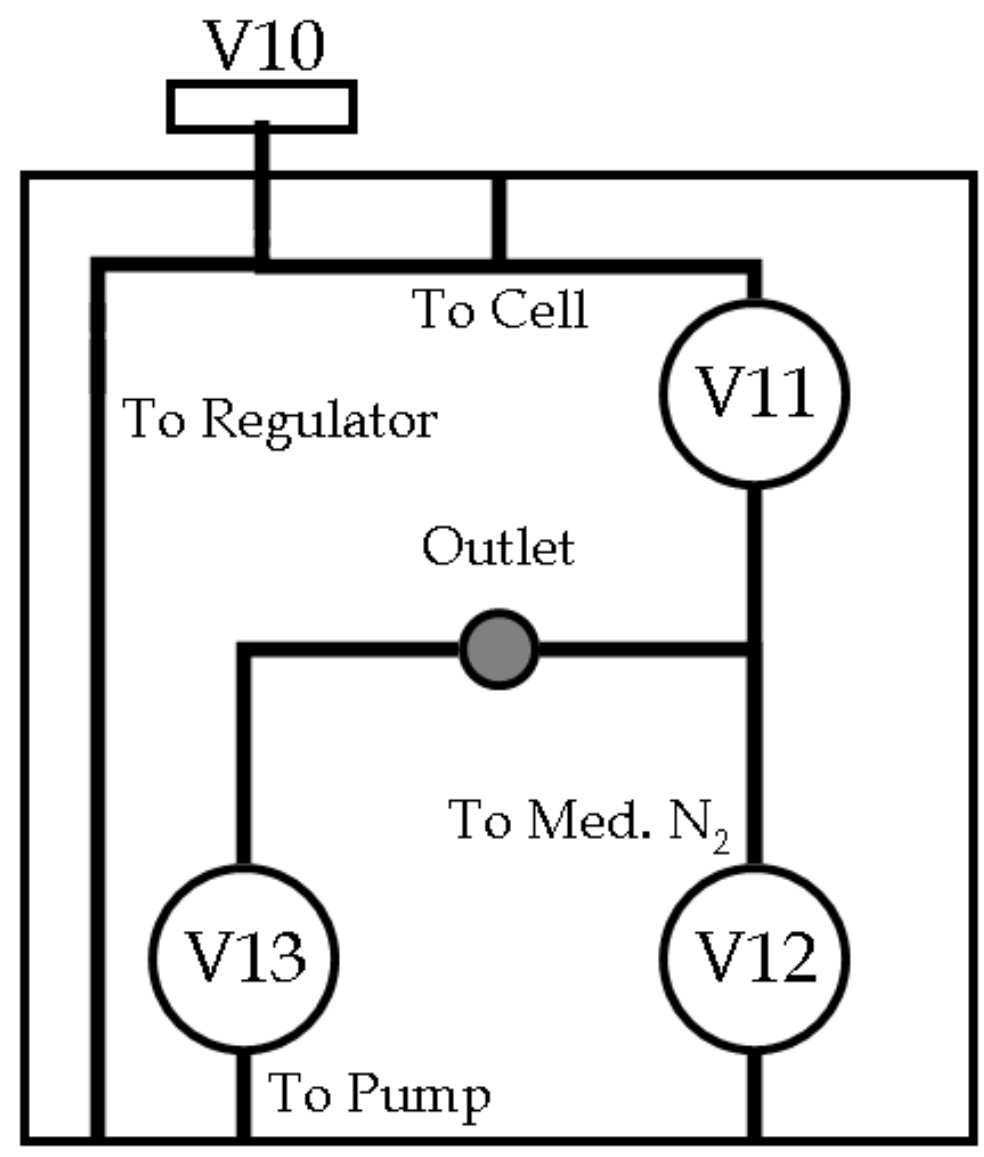

Figure B.2: Upper Gas System 


\section{B.2 Electronics}

In this section, I have provided a list of the electronics components installed in the Hybrid Polarizer, including the default settings. In addition, the equations used by the LabVIEW programs for fitting are listed below.

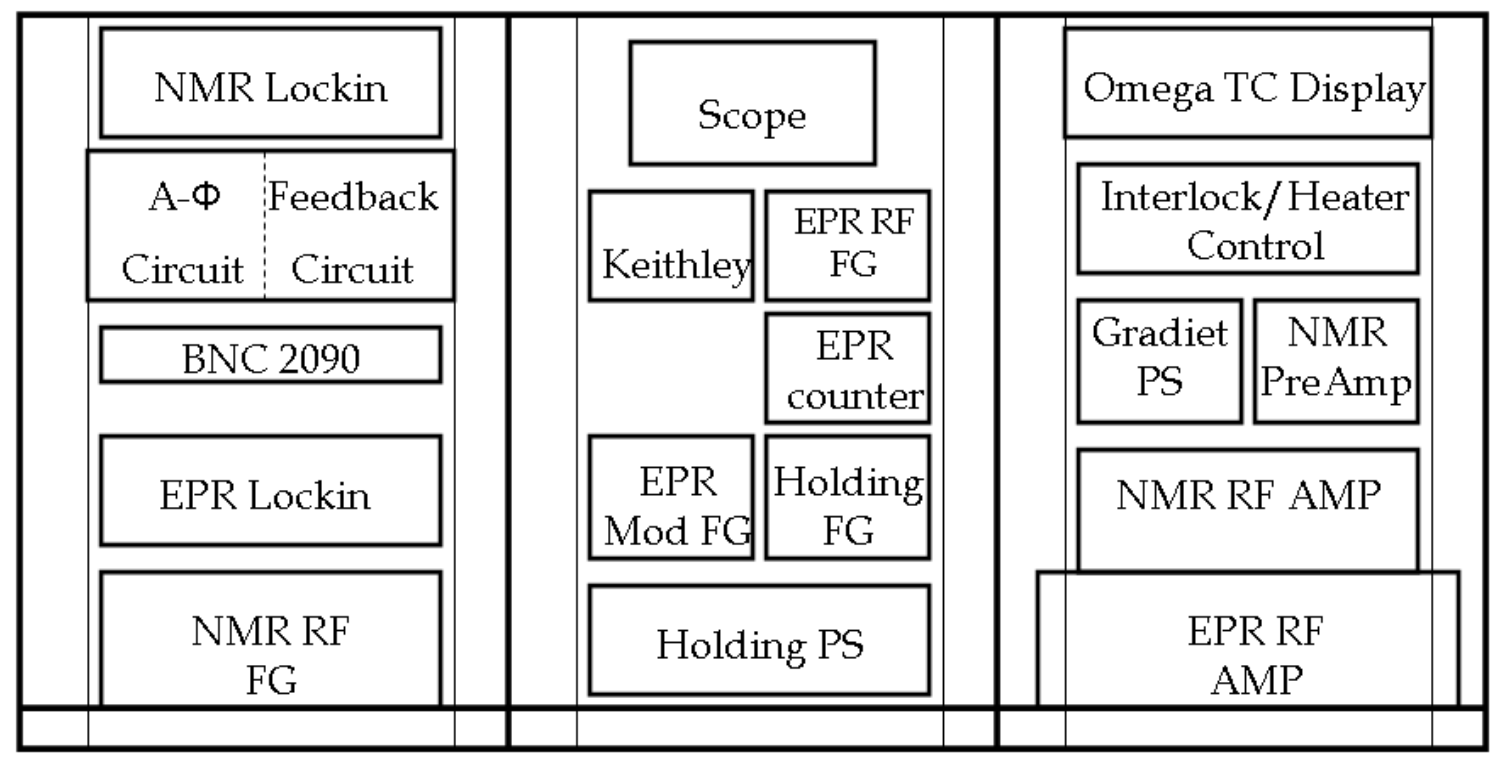

Figure B.3: Hybrid Polarizer Electronics Rack 
Table B.1: Electronics Settings

\begin{tabular}{|c|c|c|c|}
\hline Device Name & Manufacturer Name & State & Settings \\
\hline NMR Lockin & SRS SR830 DSP & Always ON & $\begin{array}{l}\text { A- NMR Pre-Amp } \\
\& \text { Scope; B- A- } \\
\Phi \text { Direct Out \& } \\
\text { Scope; A-B, AC } \\
\text { Coupling, Ground; } \\
\tau=10 m s, \quad 6 d b / O c t ; \\
\text { Sens=50mV, Reserve: } \\
\text { Normal; Filters- Line, } \\
\text { LineX2; Ch.1-X, Ch.2- } \\
\text { Y, Output- X \& Y; Ref } \\
\text { IN- NMR RF FG } \\
\text { SYNC Out, Pos Edge }\end{array}$ \\
\hline A- $\Phi$ Circuit & None & Always ON & $\begin{array}{l}\text { Sig. Gen. IN- NMR } \\
\text { RF FG OUT; Direct } \\
\text { Out- NMR Lockin \& } \\
\text { Scope; Direct Out; } \\
\text { (Lead/Lag and Gain } \\
\text { subject to change) }\end{array}$ \\
\hline Feedback Circuit & None & Always ON & $\begin{array}{l}\text { Circuit 1: unused; } \\
\text { Circuit 2: } V_{\text {in }^{-}} \text {un- } \\
\text { plugged (except dur- } \\
\text { ing EPR Take Data); } \\
\text { INT- Off (except dur- } \\
\text { ing EPR Take Data); } \\
\text { Rel. Gain- 1.5; Total } \\
\text { Gain- 5.0; } V_{\text {mod }^{-}} \text {EPR } \\
\text { MOD FG Out; } V_{\text {out }} \\
\text { 154:1 Divider to EPR } \\
\text { RF FG MOD input }\end{array}$ \\
\hline BNC 2090 & NI BNC 2090 & Always ON & $\begin{array}{l}\text { Analog Inputs: AI2- } \\
\text { Oven Temp. AI3- } \\
\text { EPR Lockin X Out- } \\
\text { put; AI4- EPR Lockin } \\
\text { Y Output (Always } \\
\text { use a BNC terminator } \\
\text { on the input below } \\
\text { the one that you are } \\
\text { using to avoid large } \\
\text { floating voltages.) }\end{array}$ \\
\hline
\end{tabular}




\begin{tabular}{|c|c|c|c|}
\hline Device Name & $\begin{array}{l}\text { Manufacture } \\
\text { Name }\end{array}$ & State & Settings \\
\hline EPR Lockin & $\begin{array}{ll}\text { SRS } & \text { SR830 } \\
\text { DSP } & \end{array}$ & Always ON & $\begin{array}{l}\text { A-Photodiode; A, AC } \\
\text { Coupling, Float; } \tau=100 m s \text {, } \\
6 d b / \text { Oct; Sens }=100 \mu \mathrm{V} \text { Re- } \\
\text { serve: Low-Noise; Filters- } \\
\text { Line, LineX2; Ch.1-X, } \\
\text { Ch.2-Y, Output- X \& Y; Ref } \\
\text { IN- EPR MOD FG SYNC, } \\
\text { Pos Edge }\end{array}$ \\
\hline NMR RF FG & HP 3325A & Always ON & $\begin{array}{l}\text { Settings controlled by } \\
\text { computer: Freq }=100 \mathrm{kHz} \text {, } \\
\text { Amp }=550 \mathrm{mV}\end{array}$ \\
\hline Scope & $\begin{array}{l}\text { Tektronics } \\
\text { TDS 2012B }\end{array}$ & Always ON & $\begin{array}{l}\text { Ch. 1- A- } \Phi \text { Direct Out; Ch. } \\
\text { 2- NMR Lockin A; Trigger- } \\
\text { Ch. 1; AC Coupling; } \\
M=10 \mu s ; A m p=100 m V\end{array}$ \\
\hline Keithley & $\begin{array}{l}\text { Keithley } \\
\text { Multimeter } \\
2000\end{array}$ & Always ON & $\begin{array}{l}\text { Rear Input from Shunt Re- } \\
\text { sistor; if display is frozen, } \\
\text { push LOCAL }\end{array}$ \\
\hline EPR RF FG & Fluke 80 & $\begin{array}{l}\text { OFF unless } \\
\text { doing EPR }\end{array}$ & $\begin{array}{l}\text { VCO Mode; settings con- } \\
\text { trolled by computer: Freq } \\
\sim 20 M H z \text {, Amp } \sim 2 V\end{array}$ \\
\hline EPR Counter & $\begin{array}{l}\text { Agilent } \\
\text { 53131A }\end{array}$ & Always ON & $\begin{array}{l}\text { Ch. 1- EPR RF FG SYNC; } \\
\text { Gate Time }=50 \mathrm{~ms}\end{array}$ \\
\hline EPR MOD FG & $\begin{array}{l}\text { Agilent } \\
33220 \mathrm{~A}\end{array}$ & Always ON & $\begin{array}{l}\text { Output- } \mathrm{V}_{M O D} \text { on Feed- } \\
\text { back Circuit; SYNC- EPR } \\
\text { Lockin REF IN; settings } \\
\text { controlled by computer: } \\
\text { Freq }=200 \mathrm{~Hz} \text {, Amp }=1.0 V_{p p}\end{array}$ \\
\hline Holding Field FG & $\begin{array}{l}\text { Agilent } \\
33220 \mathrm{~A}\end{array}$ & Always ON & $\begin{array}{l}\text { Output- Kepco Voltage } \\
\text { Programming Input; } \\
\text { settings controlled by } \\
\text { computer: Ramp, Burst, } \\
\text { Manual Trigger }\end{array}$ \\
\hline
\end{tabular}




\begin{tabular}{|c|c|c|c|}
\hline Device & Manufactures & State & Settings \\
\hline Name & Name & & \\
\hline $\begin{array}{l}\text { Holding } \\
\text { Field Power } \\
\text { Supply }\end{array}$ & $\begin{array}{l}\text { Kepco } \\
36 \mathrm{~V} / 12 \mathrm{~A} \\
\mathrm{BOP}\end{array}$ & Always ON & $\begin{array}{l}\text { Voltage and Current Con- } \\
\text { trol OFF; Voltage Mode; } \\
\text { Voltage Programming } \\
\text { Input- Holding Field FG; } \\
\text { Rear Output to coils and } \\
\text { shunt; positive voltage } \\
\text { from FG gives negative } \\
\text { voltage in Power Supply } \\
\text { which creates positive } \\
\text { Field }\end{array}$ \\
\hline $\begin{array}{l}\text { Omega } \\
\text { Tempera- } \\
\text { ture Display }\end{array}$ & None & Always ON & $\begin{array}{l}\text { 1- Heater A; 2- Heater B; 3- } \\
\text { Cell Stem }\end{array}$ \\
\hline $\begin{array}{l}\text { Interlock } \\
\text { /Heater } \\
\text { Control }\end{array}$ & None & Always ON & $\begin{array}{l}\text { Inputs- Oven temp, Com- } \\
\text { biner temp, Cube temp, } \\
\text { Flowmeters; Outputs- } \\
\text { Heaters A and B, Oven } \\
\text { temp (analogue), Laser } \\
\text { Interlock }\end{array}$ \\
\hline $\begin{array}{l}\text { Gradient } \\
\text { Coil Power } \\
\text { Supply }\end{array}$ & $\begin{array}{l}\text { BK } \quad \text { Preci- } \\
\text { sion } 1621 \mathrm{~A}\end{array}$ & Always ON & $\begin{array}{l}\text { Wire labelled }(+) \text { plugged } \\
\text { into }(+) ;(-) \text { and GND } \\
\text { shorted;Current }=2.5 \mathrm{~A}\end{array}$ \\
\hline $\begin{array}{l}\text { NMR Pre- } \\
\text { Amp }\end{array}$ & SRS SR560 & Always ON & $\begin{array}{l}\text { A- Pick-Up A, B- Pick- } \\
\text { Up B, A-B; } 50 \Omega \text { Output- } \\
\text { NMR Lockin A; Bandpass } \\
\text { Filter: 10kHz - 300kHz; } \\
\text { Low Noise, 50x Gain, Line } \\
\text { Power }\end{array}$ \\
\hline $\begin{array}{ll}\text { NMR } & \text { RF } \\
\text { AMP } & \end{array}$ & $\begin{array}{lr}T \quad \& & C \\
\text { Power } & A G \\
\text { Series } & \end{array}$ & Always ON & $\begin{array}{l}\text { Input- NMR RF FG; } \\
\text { Output- NMR RF Coils; } \\
\text { RF Output- ON; Freq- } \\
0.100 M H z ; \quad \text { Power- } 40 \% \text {; } \\
\text { Mode- MGC; Source- EXT }\end{array}$ \\
\hline $\begin{array}{ll}\text { EPR } & \text { RF } \\
\text { AMP } & \end{array}$ & AR Amp & $\begin{array}{l}\text { OFF except } \\
\text { when doing } \\
\text { EPR }\end{array}$ & $\begin{array}{lccc}\text { Input- } & \text { EPR } & \text { RF } & \text { RG, } \\
\text { Output- } & \text { EPR } & \text { RF } & \text { Coils; } \\
\text { Gain } \sim 3.5 & & \end{array}$ \\
\hline
\end{tabular}




\section{B.3 LabVIEW Programs}

This section gives some information about the LabVIEW programs used in operating the Hybrid Polarizer. Shortcuts to the vis can be found in the Desktop Folder hybrid_polarizer_vis. Most end in_polarizer because they were editted from a version created in physics. Many begin with $j$ because they were created by Jaideep Singh. If you wish to make any changes to a $v i$, append a number the the file name and save it as a new $v i$.

\section{B.4 NMR}

\begin{tabular}{|c|l|}
\hline Program & Description \\
\hline jmultnmr_polarizer.vi & $\begin{array}{l}\text { Controls DAQ for NMR and records the } \\
\text { oven temperature. This vi has the } \\
\text { ability to take a single NMR or mul- } \\
\text { tiple NMRs in the form of a Spin Up } \\
\text { or Spin Down. Saves 4 files for each } \\
\text { sweep, the .txt file is used in the } \\
\text { jnmronedata_polarizer.vi program. }\end{array}$ \\
\hline jfieldtools_polarizer.vi & $\begin{array}{l}\text { Sets parameters for NMR Sweeps and } \\
\text { Holding Field Value. }\end{array}$ \\
\hline jnmronedata_polarizer.vi & $\begin{array}{l}\text { Fits peaks from an NMR Sweep and can } \\
\text { save files for a Spin Up or Spin Down Fit. }\end{array}$ \\
\hline nmrspinupfitter.vi & $\begin{array}{l}\text { Reads the file generated by } \\
\text { jnmronedata_polarizer.vi and fits to } \\
\text { an exponential curve, giving the saturation } \\
\text { signal and Spin Up Time Constant. }\end{array}$ \\
\hline
\end{tabular}




\section{B.5 EPR}

\begin{tabular}{|c|l|}
\hline \multicolumn{1}{|c|}{ Program } & Description \\
\hline jfmsweep_polarizer.vi & $\begin{array}{l}\text { First program used in EPR to locate the al- } \\
\text { kali transition frequency. Saves a file with } \\
\text { VCO freq, Counter freq, and EPR Lockin X } \\
\text { \&Y. }\end{array}$ \\
\hline jeprtakedata_polarizer.vi & $\begin{array}{l}\text { Controls the NMR FG and records the data } \\
\text { during the EPR spin flip. Saves a file with } \\
\text { the Counter freq, and counts. }\end{array}$ \\
\hline jepranalrbk_polarizer.vi & $\begin{array}{l}\text { Calculates }{ }^{3} H e \text { polarization from fre- } \\
\text { quency shift data. Uses the file generated } \\
\text { by jeprtakedata_polarizer.vi, } \\
\text { and density, temperature, and volume } \\
\text { information provided by the user. }\end{array}$ \\
\hline
\end{tabular}

\section{B.6 Other}

\begin{tabular}{|c|l|}
\hline Program & Description \\
\hline nmrspindownfitter.vi & $\begin{array}{l}\text { Reads the file generated by } \\
\text { jnmronedata_polarizer.vi and } \\
\text { fits to an exponential curve, giving a cell } \\
\text { lifetime and starting polarization. }\end{array}$ \\
\hline alkalipolarization.vi & $\begin{array}{l}\text { Takes data and controls settings for the al- } \\
\text { kalipolarization sweep. }\end{array}$ \\
\hline pol_fitter.vi & $\begin{array}{l}\text { Fits the data from } \\
\text { alkalipolarization.vi and gives } \\
\text { an alkali polarization. }\end{array}$ \\
\hline
\end{tabular}




\section{B.7 EPR Equations}

The size of the shift in the alkali metal EPR frequency can be directly converted into a helium polarization using the following formulas [58]:

$$
\begin{gathered}
B_{H e}=\frac{B_{\uparrow \uparrow}-B_{\uparrow \downarrow}}{2} \\
B_{H e}=\frac{-b-s * \sqrt{b^{2}-4 c}}{2} \\
b=-\frac{g \mu_{B}\left(\frac{A}{2 k+1}-s h * \Delta v_{H e}\right)-g_{K} \mu_{N}\left(\frac{2 k A}{2 k+1}-s h * \Delta v_{H e}\right)}{s\left(g \mu_{B}\right)\left(g K \mu_{N}\right)} \\
c=\frac{s h * \Delta v_{H e}\left(A-s h * \Delta v_{H e}\right)}{g \mu_{B} g_{K} \mu_{N}} \\
B_{H e}=\frac{2 \mu_{0}}{3} M_{H e}, \quad M_{H e}=\kappa_{0} *\left[{ }^{3} H e\right] g \mu_{N} \frac{P_{H e}}{2}
\end{gathered}
$$

\begin{tabular}{|c|c|c|c|}
\hline Constant & Value & Constant & Value \\
\hline $\mathrm{h}$ & $6.626^{*} 10^{-34} \mathrm{~J} \cdot \mathrm{s}$ & $\mathrm{g}$ & 2.002 \\
\hline $\mathrm{hfs}$ & 461.71972 & $\mathrm{k}$ & $3 / 2$ \\
\hline $\mathrm{s}$ & $-1($ or 1$)$ & $\mathrm{mm}$ & 0.39146 \\
\hline$g_{K}$ & $\mathrm{~mm} / \mathrm{k}$ & $\mathrm{A}$ & $\mathrm{hfs}^{*} 10^{6} * h$ \\
\hline$\kappa_{00}$ & 4.541 & $\kappa_{0 T}$ & 0.00924 \\
\hline$\mu_{H e}$ & $-2.12762 * \mu_{N}$ & $\kappa_{0}$ & $\kappa_{00}+\kappa_{0 T} * T$ \\
\hline
\end{tabular}

Table B.2: Values are given for ${ }^{39} \mathrm{~K}$

Once the helium-3 magnetic field strength $B_{H e}$ has been calculated, it can be directly converted into a helium-3 polarization $P_{\mathrm{He}}$ using

$$
P_{\mathrm{He}}=\frac{2}{\kappa_{0} *\left[{ }^{3} \mathrm{He}\right]} * \frac{3 * B_{\mathrm{He}}}{2 \mu_{0}}
$$

where $\mu_{0}$ is the permeability of free space, $\left[{ }^{3} \mathrm{He}\right]$ is the density of helium-3, and $\kappa_{0}$ 
is the empirical shift constant

$$
\kappa_{0}=\kappa_{00}+\kappa_{0 T} * T
$$

$\kappa_{0}$ has both a temperature-independent contribution $\kappa_{00}$ and a temperature-dependent contribution $\kappa_{0 T}$

$$
\kappa_{00}=4.541, \quad \kappa_{0 T}=0.00924
$$




\section{Bibliography}

[1] P. C. Lauterbur, "Image formation by induced local interactions: Examples employing nuclear magnetic resonance," Nature, vol. 242, pp. 190-191, 1973.

[2] M. Albert, G. D. Cates, B. Driehuys, W. Happer, B. Saam, C. Spinger Jr., and A. Wishnia, "Biological magnetic resonance imaging using laser-polarized 129xe," Nature, vol. 370, no. 1, pp. 199-201, 1994.

[3] E. E. de Lange, T. A. Altes, J. T. Patrie, B. J. J., J. A. P., J. P. Mugler III, and T. A. Platts-Mills, "Changes in regional airflow obstruction over time in the lungs of patients with asthma: evaluation with 3he mr imaging," Radiology, vol. 250, no. 2, pp. 567-575, 2009.

[4] Y. Tzeng, K. Lutchen, and M. Albert, "The difference in ventilation heterogeneity between asthmatic and healthy subjects quantified using hyperpolarized 3he mri," J. Appl. Physiol., vol. 106, pp. 813-822, 2009.

[5] P. Koumellis, E. van Beek, and N. Woodhouse, "Quantitative analysis of regional airways obstruction using dynamic hyperpolarized 3he mri- preliminary results in children with cystic fibrosis," J. Magn. Reson. Imag., vol. 22, pp. 420-426, 2005.

[6] E. van Beek, A. Dahmen, and T. Stavngaard, "Hyperpolarized 3he mri versus 
hrct in copd and normal volunteers: Phil trail," Eur. Respir. J., vol. 34, pp. 1311$1321,2009$.

[7] N. Woodhouse, J. M. Wild, and M. Paley, “Combined helium-3/proton magnetic resonance imaging measurement of ventilated lung volumes in smokers compared to never-smokers," J. Mag. Reson. Imag., vol. 21, pp. 365-369, 2005.

[8] A. Lopez, K. Shibuya, C. Rao, M. C.D., A. Hansell, L. Held, V. Schmid, and S. Buist, "Chronic obstructive pulmonary disease: current burden and future projections," Eur. Respir J., vol. 27, pp. 397-412, 2006.

[9] U. D. of Health and H. Services, "Chronic obstructive pulmonary disease," USDHHS Data Fact Sheet, 2003.

[10] J. West, Respiratory Physiology- The Essentials, 6th Edition. Baltimore, MD: Lippincott Williams \& Wilkins, 2000.

[11] D. Mannino and V. A. Kiri, "Changing the burden of copd mortality," Intl J Chron Obstruc Pulmon Dis, vol. 1, pp. 219-233, 2006.

[12] M. Levitzky, Pulmonary Physiology, 5th Edition. New York, NY: The McgrawHill Companies, 1999.

[13] M. Salerno and T. Altes, “Emphysema: Hyperpolarized helium 3 diffusion mr imaging of the lungs compared with spirometric indexes-initial experience," Radiology, vol. 222, pp. 252-260, 2002.

[14] J. F. Mata, T. A. Altes, and G. D. Cates Jr., “Evaluation of emphysema severity and progression in a rabbit model: comparison of hyperpolarized 3he and 129xe diffusion mri with lung morphometry," J Appl Physiol, vol. 102, pp. 1273-1280, 2007. 
[15] K. Emami, R. Cadman, J. M. Woodburn, M. Fischer, S. Kadlecek, J. Zhu, S. Pickup, R. Guyer, M. Law, V. Vahdat, M. Friscia, M. Ishii, J. Yu, W. Gefter, J. Shrager, and R. Rizi, "Early changes of lung function and structure in an elastase model of emphysema-a hyperpolarized 3he mri study," J. Appl. Physiol., vol. 104, pp. 773-786, 2008.

[16] T. Stavngaard, L. V. Sogaard, M. Batz, L. Schreiber, and A. Dirksen, "Progression of emphysema evaluated by mri using hyperpolarized 3he measurements in patients with alpha-1-antitrypsin (a1at) deficiency compared with ct and lung function tests," Acad. Radiology, vol. 9, pp. 1019-1026, 2009.

[17] L. Mathew, M. Kirby, R. Etemad-Rezai, A. Wheatley, D. G. McCormack, and G. Parraga, "Hyperpolarized 3he magnetic resonace imaging: Preliminary evaluation of phenotyping potential in chronic obstructive pulmonary disease," Eur. J of Rad., vol. 79, pp. 140-146, 2011.

[18] R. L. O'Halloran, J. H. Holmes, Y.-C. Wu, A. Alexander, and S. B. Fain, "Helium-3 mr q-space imaging with radial acquisition and iterative highly constrained back-projection," MRM, vol. 63, pp. 41-50, 2010.

[19] G. J. Stanisz, E. E. Odrobina, J. Pun, M. Escaravage, S. J. Graham, M. J. Bronskill, and R. M. Henkelman, "T1, t2 relaxation and magnetization transfer in tissue at 3t," MRM, vol. 54, pp. 507-512, 2005.

[20] M. Salerno, J. R. Brookeman, E. E. de Lange, and J. P. Mugler III, “Hyperpolarized 3he lung imaging at 0.5 and 1.5 tesla: A study of susceptibilityinduced effects," MRM, vol. 53, pp. 212-216, 2005.

[21] M. H. Deppe, J. Parra-Robles, S. Ajraoui, S. R. Parnell, M. Clemence, R. F. 
Schulte, and J. M. Wild, "Susceptibility effects in hyperpolarized 3he lung mri at 1.5t and 3t," JMRI, vol. 30, pp. 418-423, 2009.

[22] J. Mugler III, I. Dregely, T. Altes, S. Ketel, I. Ruset, J. Distelbrink, Y. Chang, J. Mata, F. Hersman, and K. Ruppert, "T2* for hyperpolarized xe129 in the healthy human lung at 1.5t and 3t," Proc. Intl. Soc. Mag. Reson. Med., vol. 17, no. 2207, 2009.

[23] M. A. Bernstein, K. F. King, and X. J. Zhou, Handbook of MRI Pulse Sequences. Burlington, MA: Elsevier Acad. Press, 2004.

[24] E. Hahn, “Spin echoes," Phys. Rev., vol. 80, p. 580, 1950.

[25] J. P. Mugler III, T. Altes, I. C. Ruset, I. M. Dregely, J. F. Mata, G. W. Miller, S. Ketel, J. Ketel, W. F. Hersman, and K. Ruppert, "Simultaneous magnetic resonance imaging of ventilation distribution and gas uptake in the human lung using hyperpolarized xenon-129," PNAS, vol. 107, no. 50, pp. 2170721712, 2010.

[26] G. A. Johnson, G. Cates, X. J. Chen, G. P. Cofer, B. Driehuys, W. Happer, L. W. Hedlund, B. Saam, M. D. Shattuck, and J. Swartz, "Dynamics of magnetization in hyperpolarized gas mri of the lung," MRM, vol. 38, pp. 66-71, 1997.

[27] C. H. Hayes, W. Edelstein, J. Schenck, O. Mueller, and M. Eash, “An efficient, highly homogeneous radiofrequency coil for whole-body $\mathrm{nmr}$ imaging at 1.5t," JMR, vol. 63, pp. 622-628, 1985.

[28] T. Altes, P. Powers, and J. Knight-Scott, “Hyperpolarized 3he mr lung ventilation imaging in asthmatics: preliminary findings," J. Magn Reson., vol. 13, pp. 378-384, 2001. 
[29] E. Stejskal and J. Tanner, "Spin diffusion measurements: Spin echoes in the presence of a time-dependent field gradient," J. Chem. Phys., vol. 42, p. 288, 1965.

[30] P. P. Mitra and P. N. Sen, “Effects of microgeometry and surface relaxation on nmr pulsed-field-gradient experiments: Simple pore geometries," Phys. Rev. B, vol. 45, pp. 143-156, 1992.

[31] P. P. Mitra, P. N. Sen, and L. M. Schwartz, "Short-time behavior of the diffusion coefficient as a geometrical probe of porous media," Phys. Rev. B, vol. 47, pp. 8565-8574, 1993.

[32] P. Sen, L. M. Schwartz, P. P. Mitra, and B. I. Halperin, "Surface relaxation and the long-time diffusion coefficient in porous media: Periodic geometries," Phys Rev B, vol. 49, pp. 215-225, 1994.

[33] R. W. Mair, D. G. Cory, S. Peled, and et al, "Pulsed-field-gradient measurements of time-dependent gas diffusion," J Magn Reson., vol. 135, pp. 478-486, 1998.

[34] R. W. Mair, G. P. Wong, D. Hoffman, and et al, "Probing porous media with gas diffusion nmr," Phys. Rev. Lett,, vol. 83, pp. 3324-3327, 1999.

[35] R. W. Mair, P. N. Sen, and S. Patz, "The narrow pulse approximation and long length scale determination in xenon gas diffusion nmr studies of model porous media," J Magn Reson., vol. 156, pp. 202-212, 2002.

[36] M. Carl, G. W. Miller, and G. D. Cates Jr., “Measurement of hyperpolarized gas diffusion at very short time scales," J Magn Reson., vol. 189, no. 2, pp. 228240, 2007. 
[37] M. Carl, Characterizing Microstructure of Porous Media Using Noble-GasDiffusion MRI at Short Time Scales. PhD thesis, University of Virginia, Charlottesville VA, 2008.

[38] M. A. Bouchiat, T. Carver, and V. C., "Nuclear polarization in 3he gas induced by optical pumping and dipolar exchange," Phys. Rev. Letters, vol. 5, pp. 373$375,1960$.

[39] F. Colegrove, "Polarization of he-3 gas by optical pumping," Phys. Rev., vol. 132, p. 2561, 1963.

[40] H.-U. Kauczor, "Hyperpolarized helium-3 gas magnetic resonance imaging of the lung," Topics in MRI, vol. 14, no. 3, pp. 223-230, 2003.

[41] D. Kramer, “Doe begins rationing helium-3," Physics Today, vol. 63, no. 6, 2010.

[42] P. A. M. Dolph, High-Performance Nuclear-Polarized 3He Targets for Electron Scattering Based on Spin-Exchange Optical Pumping. PhD thesis, University of Virginia, Charlottesville VA, 2010.

[43] J. Singh, P. Dolph, K. Mooney, V. Nelyubin, W. Tobias, A. Kelleher, T. Averett, and G. Cates, "Recent advances in polarized he-3 targets," AIP Conf. Proc., vol. 1149, p. 823, 2009.

[44] W. Happer and A. Tam, "Effect of rapid spin exchange on the magneticresonance spectrum of alkali vapors," Phys. Rev. A, vol. 16, pp. 1877-1891, 1977.

[45] S. Applet, B. A. B.-A., C. Erickson, M. Romalis, A. Young, and W. Happer, "Theory of spin-exchange optical pumping of 3he and 129xe," Phys. Rev. A, vol. 58, pp. 1412-1439, 1998. 
[46] A. Ben-Amar Baranga, S. Appelt, M. V. Romalis, C. J. Erickson, A. R. Young, G. D. Cates, and W. Happer, "Polarization of 3he by spin exchange with optically pumped rb and k vapors," Phys. Rev. Lett., vol. 80, no. 13, pp. 2801-2804, 1998.

[47] T. E. Chupp, E. R. Oteiza, J. M. Richardson, and T. R. White, "Precision frequency measurements with polarized he3, ne21, and xe129 atoms," Phys. Rev. A, vol. 38, no. 8, pp. 3998-4003, 1988.

[48] T. E. Chupp, R. A. Loveman, A. K. Thompson, A. M. Bernstein, and D. R. Tieger, "Tests of a high density polarized he3 target for electron scattering," Phys. Rev. C, vol. 45, no. 3, pp. 915-930, 1992.

[49] X. Riordan and et al, "Measurements of the electric form factor of the neutron up to q-squared $=3.4$ gev-squared using the reaction 3he(e, e'n)pp," Phys. Rev. Lett., vol. 105, no. 26, pp. 262302:1-5, 2010.

[50] B. Chann, E. Babcock, L. W. Anderson, T. G. Walker, W. C. Chen, T. B. Smith, A. K. Thompson, and T. R. Gentile, "Production of highly polarized 3he using spectrally narrowed diode laser array bars," Journal of Applied Physics, vol. 94, no. 10, pp. 6908-6914, 2003.

[51] J. Singh, “Uva progress report," tech. rep., Jefferson Lab, April 2005. Biweekly collaboration meeting.

[52] V. Nelyubin, "Gaussian optics simulations for hybrid polarizer," tech. rep., University of Virginia, 2008.

[53] W. Happer, G. Cates, M. Romalis, and C. Erickson, “U.s. patent no. 6,318,092,” 2001. 
[54] E. Babcock, I. Nelson, S. Kadlecek, B. Driehuys, L. W. Anderson, F. W. Hersman, and T. G. Walker, "Hybrid spin-exchange optical pumping of 3he," Phys. Rev. Lett., vol. 91, no. 12, pp. 123003-1-4, 2003.

[55] A. Abragam, Principles of Nuclear Magnetism. Oxford University Press, Oxford, 1961.

[56] M. V. Romalis and G. D. Cates, "Accurate 3he polarimetry using rb zeeman frequency shift due to the rb-3he spin-exchange collisions.," Phys. Rev. A, vol. 58, no. 4, pp. 3004-3011, 1998.

[57] G. D. Cates, D. J. White, T.-R. Chien, S. R. Schaefer, and W. Happer, "Spin relaxation in gases due to inhomogeneous static and oscillating magnetic fields," Phys. Rev. A, vol. 38, no. 10, pp. 5092-5106, 1988.

[58] J. Singh, Alkali-Hybrid Spin-Exchange Optically-Pumped Polarized 3He Targets Used for Studying Neutron Structure. PhD thesis, University of Virgina, Charlottesville VA, 2010.

[59] E. Babcock, I. Nelson, S. Kadlecek, and T. G. Walker, "3he polarizationdependent epr frequency shifts of alkali-metal-3he pairs," Phys. Rev. A, vol. 71, no. 1, pp. 013414-013418, 2005.

[60] B. C. Grover, "Noble-gas nmr detection through noble-gas-rubidium hyperfine contact interaction," Phys. Rev. Lett., vol. 40, no. 6, pp. 391-392, 1978.

[61] L. Krause, "Collisional excitation transfer between $2 p-1 / 2$ and 2p-3/2 levels in alkali atoms.," Appl. Opt., vol. 5, no. 9, pp. 1375-1382, 1966.

[62] N. R. Newbury, P. Bogorad, G. D. Cates, M. Gatzke, H. Mabuchi, and B. Saam, "Polarization-dependent frequency shifts from rb-3he collisions," Phys. Rev. A, vol. 48, no. 1, pp. 558-568, 1993. 
[63] M. Romalis, Laser Polarized 3He Target used for a Precision Measurement of the Neutron Spin Structure. PhD thesis, Princeton University, Princeton NJ, 1997.

[64] A. Oppelt and et al, "Fisp- a new fast mri sequence," Electromedia, vol. 54, pp. 15-18, 1986.

[65] E. M. Haacke, R. W. Brown, M. R. Thompson, and R. Venkatesan, Magnetic Resonance Imaging: physical principles and sequence design. John Wiley and Sons, Inc., 1999.

[66] J. Mugler III, M. Salerno, E. de Lange, and J. Brookeman, “Optimized truefisp hyperpolarized 3he mri of the lung yields a 3-fold snr increase compared to flash," Proc. Intl. Soc. Mag. Reson. Med., vol. 10, no. 2019, 2002.

[67] J. M. Wild and et al, "Steady-state free precession with hyperpolarized 3-he: Experiments and theory," J. Mag. Reson., vol. 183, pp. 13-24, 2006.

[68] O. Bieri, M. Markl, and K. Scheffler, "Analysis and compensation of eddy currents in balanced ssfp," MRM, vol. 54, pp. 129-137, 2005.

[69] K. Scheffler, S. Maderwald, M. E. Ladd, and O. Bieri, "Oscillating steady states," Mag. Reson. in Med., vol. 55, pp. 598-603, 2006.

[70] R. Buxton, "The diffusion sensitivity of fast steady-state free precession imaging," Mag. Reson. in Med., vol. 29, pp. 235-243, 1993.

[71] R. Bruning and et al, "Diffusion measurements in the ischemic human brain with a steady-state sequence," Investigative Radiology, vol. 31, pp. 709-715, 1996.

[72] J. McNab and K. Miller, "Sensitivity of diffusion weighted steady state free 
precession to anisotropic diffusion," Mag. Reson. in Med., vol. 60, pp. 405-413, 2008.

[73] J. McNab and K. Miller, "Steady-state diffusion-weighted imaging: theory acquisition and analysis," NMR in Biomed., vol. 23, pp. 781-793, 2010.

[74] C. H.O., R. Rogers, K. Whittall, Y. D'Yachkova, P. Pare, F. Sciurba, and J. Hogg, "A quantification of lung surface area in emphysema using computed tomography," Amer. J. Respir. Crit. Care Med., vol. 159, pp. 851-856, 1999.

[75] P. Sen, "Time-dependent diffusion coefficient as a probe of geometry," Concepts in MR Part A, vol. 23A, pp. 1-21, 2004.

[76] L. Latour, P. Mitra, R. Kleinberg, and C. Sotak, “Time-dependent diffusion coefficient of fluids in porous media as a probe of surface-to-volume ratio," JMR, vol. 101, pp. 342-346, 1993.

[77] M. Hurlimann, K. Helmer, L. Latour, and C. Sotak, "Determination of surfacearea-to-volume ratio and surface relaxivity," JMR, vol. 111, pp. 169-178, 1994.

[78] X. Chen, H. Moller, M. Chawla, G. Cofer, B. Driehuys, L. Hedlund, and G. Johnson, "Spatially resolved measurements of hyperpolarized gas properties in the lung in vivo, part i: diffusion coefficient," MRM, vol. 42, pp. 721$728,1999$.

[79] M. Bock, "Simultaneous t2* and diffusion measurements with 3he," MRM, vol. 38, pp. 890-895, 1997.

[80] S. Torquato, T. Truskett, and P. Debenedetti, "Is random close packing of spheres well defined?," Phys. Rev. Lett., vol. 84, no. 10, pp. 2064-2067, 2000. 
[81] A. P. Wills and et al, "The magnetic susceptibility of oxygen, hydrogen, and helium," Physical Review, vol. 23, no. 2, pp. 209-220, 1924.

[82] T. A. Case, C. Durney, D. Ailion, A. Cutillo, and A. Morris, "A mathematical modelof diamagnetic line broadeningin lung tissue and similar heterogeneous systems: Calculations and measurements," JMR, vol. 73, pp. 304-314, 1987.

[83] J. Mugler III, K. Mooney, T. Altes, J. Mata, K. Qing, E. de Lange, W. Tobias, J. Brookeman, G. Cates Jr., and G. Miller, "High-quality ventilation imaging of the human lung using $100 \mathrm{ml}$ of helium-3," Proc. Intl. Soc. Mag. Reson. Med., vol. 20, no. 1357, 2012.

[84] M. V. Romalis and W. Happer, "Inhomogeneously broadened spin masers.," Phys. Rev. A, vol. 60, no. 2, pp. 1385-1402, 1999.

[85] E. Babcock, B. Chann, T. G. Walker, W. Chen, and T. Gentile Phys. Rev. Letters, vol. 96, no. 8, p. 083003, 2006. 